\title{
Organophotoredox-Catalyzed Three-Component Coupling of Heteroatom Nucleophiles, Alkenes and Aliphatic Redox Active Esters
}

\author{
Shotaro Shibutani, ${ }^{\dagger}$ Kazunori Nagao, ${ }^{* \dagger}$ and Hirohisa Ohmiya ${ }^{\star} \dagger^{\S}$
}

'Division of Pharmaceutical Sciences, Graduate School of Medical Sciences, Kanazawa University, Kakuma-machi, Kanazawa 920-1192, Japan

${ }^{\S}$ JST, PRESTO, 4-1-8 Honcho, Kawaguchi, Saitama, 332-0012, Japan

*E-mail: Kazunori Nagao: nkazunori@p.kanazawa-u.ac.jp

Hirohisa Ohmiya: ohmiya@p.kanazawa-u.ac.jp

\section{Table of Contents}

- Instrumentation and Chemicals

S2

- Characterization Data for Organophotoredox Catalysts

$\mathrm{S} 3-\mathrm{S} 4$

- Characterization Data for Redox Active Esters

$\mathrm{S} 4-\mathrm{S} 5$

- Procedure for Organophotoredox Catalyzed Decarboxylative Alkoxyalkylation of Alkene

S5

- Characterization Data for Dialkyl Ether Products

- Procedure for Three Component Coupling with Other Heteroatom Nucleophiles S19

- Characterization Data for Three Component Coupling Products

- UV-Vis Spectra of Reaction Mixtures

S28

- Color Comparison of Solutions

S29

- Stoichiometric Reaction

- Cyclic Voltammetry of Organophotocatalysts

S31

- UV-Vis Spectra of Organophotocatalysts

S32

- Fluorescence Spectra of Organophotocatalysts

S33

- Summary of Redox Properties of Organophotocatalysts

S34

- Computational Study on Gibbs Energies of Benzyl Cation and Benzyl Sulfonium

- References S39

- NMR Spectra 


\section{- Instrumentation and Chemicals}

NMR spectra were recorded on a JNM-ECS400, operating at $400 \mathrm{MHz}$ for ${ }^{1} \mathrm{H}$ NMR and 100.5 $\mathrm{MHz}$ for ${ }^{13} \mathrm{C} \mathrm{NMR}$, JNM-ECA600, operating at $600 \mathrm{MHz}$ for ${ }^{1} \mathrm{H}$ NMR and $150.9 \mathrm{MHz}$ for ${ }^{13} \mathrm{C}$ NMR, and Bruker Avance NEO 400N, operating at $400 \mathrm{MHz}$ for ${ }^{1} \mathrm{H}$ NMR and $100.6 \mathrm{MHz}$ for ${ }^{13} \mathrm{C}$ NMR. Chemical shift values for ${ }^{1} \mathrm{H}$ and ${ }^{13} \mathrm{C}$ are referenced to $\mathrm{Me}_{4} \mathrm{Si}$ and the residual solvent resonances, and for ${ }^{19} \mathrm{~F}$ is referenced to trifluorotoluene, respectively. Chemical shifts are reported in $\delta$ ppm. Mass spectra were obtained with JMS-T100TD (DART and ESI-TOF). TLC analyses were performed on commercial glass plates bearing 0.25 -mm layer of Merck Silica gel $60 \mathrm{~F}_{254}$. Silica gel (Wakosil ${ }^{\circledR} 60$, 64 210 $\mu \mathrm{m}$ ) and Aluminum oxide 90 standardized (Merck Millipore) were used for column chromatography. IR spectra were measured with a Thermo Scientific iD7 ATR Accessory for the Thermo Scientific Nicolet iS5 FT-IR Spectrometer. Melting points were measured on a Stanford Research System MPA100 (OptiMelt) apparatus. Optical rotations were measured on a JASCO P2200. CV measurements were recorded with a Hokuto Denko HZ-7000 potentiostat. UV-Vis absorption spectra were recorded on a Shimadzu UV-1900. Fluorescence spectra were recorded on a Shimadzu RF-6000. Kessil A160W Tuna Blue (highest blue and intensity setting) was used as a light source. TEKNOS MG9 was used as a fan.

All reactions were carried out under nitrogen atmosphere. Materials were obtained from commercial suppliers or prepared according to standard procedures unless otherwise noted. $\mathrm{LiBF}_{4}$ was purchased from Aldrich Chemical Co., stored under nitrogen, and used as received. Acetonitrile, THF and DCM were purchased from FUJIFILM Wako Pure Chemical Co., stored under nitrogen, and used as received. The alcohols $\mathbf{1}$ were obtained from commercial suppliers. The alkenes $\mathbf{2 a - 2 d}$,

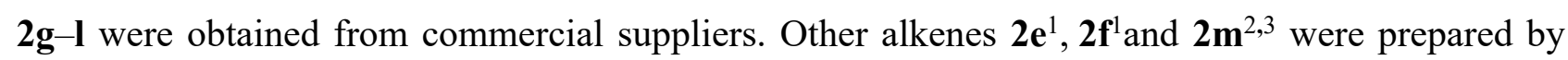
Wittig reaction from the corresponding aldehydes. The redox active esters $\mathbf{3} \mathbf{a}^{4}, \mathbf{3 b}^{5}, \mathbf{3 c}^{6}, \mathbf{3 f}^{4}, \mathbf{3 g}^{7}, \mathbf{3 i}^{8}$ and $\mathbf{3} \mathbf{j}-\mathbf{l}^{4}$ were prepared by the reported procedure. ${ }^{9}$ The carboxylc acids $\mathbf{5}$ were obtained from commercial suppliers. The amides $7 \mathbf{a}-\mathbf{c}$ and $\mathbf{7 e}$ were obtained from commercial suppliers. The amides $\mathbf{7 d}^{10}$ and $\mathbf{7} \mathbf{f}^{11}$ were prepared by acetylation of the corresponding anilines. Triethylamine trihydrofluoride was purchased from Tokyo Chemical Industry Co., stored under nitrogen, and used as received. Collidine $\cdot \mathrm{HCl}$ (9a) was prepared by the reported procedure. ${ }^{12}$ PTH2 were prepared by the reported procedure. ${ }^{13}$ PTH1, PTH3 and PTH4 were prepared by the Pd-catalyzed C-N coupling between the corresponding phenothiazine and aryl bromides. 


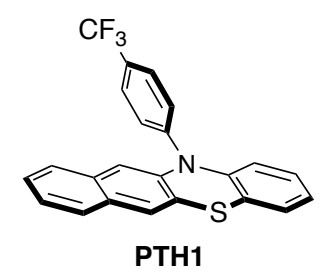

The organophotoredox catalyst PTH1 was purified by flash chromatography on silica gel (100:090:10, hexane/EtOAc). $1.0 \mathrm{mmol} \mathrm{scale;} 268 \mathrm{mg}, 0.68 \mathrm{mmol}, 68 \%$ isolated yield. Yellow solid. M.p. 143-146 ${ }^{\circ} \mathrm{C}$. IR (neat) 743, 864, 1066, 1125, 1167, 1243, 1322, $1448 \mathrm{~cm}^{-1}$. ${ }^{1} \mathbf{H}$ NMR (400 MHz, $\left.\mathrm{CDCl}_{3}\right) \delta 7.85(\mathrm{~d}, J=8.4 \mathrm{~Hz}, 2 \mathrm{H}), 7.61-7.59(\mathrm{~m}, 2 \mathrm{H}), 7.51(\mathrm{~d}, J=8.4 \mathrm{~Hz}, 2 \mathrm{H}), 7.43(\mathrm{~m}, 1 \mathrm{H})$, 7.31-7.27 (m, 2H), $7.18(\mathrm{dd}, J=7.6,1.6 \mathrm{~Hz}, 1 \mathrm{H}), 7.00-6.91(\mathrm{~m}, 2 \mathrm{H}), 6.64(\mathrm{~s}, 1 \mathrm{H}), 6.40$ (dd, $J=16.0$, $1.2 \mathrm{~Hz}, 1 \mathrm{H}) .{ }^{13} \mathbf{C}$ NMR $\left(100.6 \mathrm{MHz}, \mathrm{CDCl}_{3}\right) \delta 145.3,142.4,140.8,132.8,130.5,129.1\left(\mathrm{q},{ }^{2} J_{\mathrm{C}-\mathrm{F}}=\right.$ $32.8 \mathrm{~Hz}$ ), 128.4, 127.9 (q, $\left.{ }^{3} J_{\mathrm{C}-\mathrm{F}}=3.6 \mathrm{~Hz}\right), 127.2,127.0,127.0,126.3,126.2,125.2,125.0,124.4$, $124.0\left(\mathrm{q},{ }^{1} J_{\mathrm{C}-\mathrm{F}}=271.4 \mathrm{~Hz}\right), 123.2,122.6,118.3,113.9$. HRMS-DART $(\mathrm{m} / \mathrm{z}):[\mathrm{M}+\mathrm{H}]^{+}$calcd for $\mathrm{C}_{23} \mathrm{H}_{15} \mathrm{~F}_{3} \mathrm{NS}$, 394.0872; found, 394.0869.

12-(4-Methoxyphenyl)-12H-benzo[b]phenothiazine (PTH3)

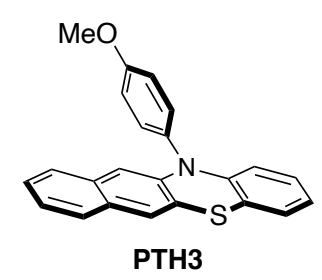

The organophotoredox catalyst PTH3 was purified by flash chromatography on silica gel (100:0 90:10, hexane/EtOAc). $0.4 \mathrm{mmol}$ scale; $114 \mathrm{mg}, 0.32 \mathrm{mmol}, 80 \%$ isolated yield. White solid. M.p. 151-156 ${ }^{\circ} \mathrm{C}$. IR (neat) 744, 862, 1034, 1245, 1447, $1508 \mathrm{~cm}^{-1} .{ }^{1} \mathbf{H}$ NMR (400 MHz, $\left.\mathrm{CDCl}_{3}\right) \delta 7.52$ $(\mathrm{m}, 1 \mathrm{H}), 7.45(\mathrm{~s}, 1 \mathrm{H}), 7.37-7.30(\mathrm{~m}, 3 \mathrm{H}), 7.22-7.16(\mathrm{~m}, 4 \mathrm{H}), 7.04(\mathrm{dd}, J=7.6,1.6 \mathrm{~Hz}, 1 \mathrm{H}), 6.85-$ $6.79(\mathrm{~m}, 2 \mathrm{H}), 6.39(\mathrm{~s}, 1 \mathrm{H}), 6.21(\mathrm{~d}, J=8.0 \mathrm{~Hz}, 1 \mathrm{H}), 3.94(\mathrm{~s}, 3 \mathrm{H}) .{ }^{13} \mathbf{C} \mathbf{N M R}\left(100.6 \mathrm{MHz}, \mathrm{CDCl}_{3}\right)$ $\delta 159.3,143.4,142.1,133.4,133.1,132.1$ (× 2C), 130.0, 126.8, 126.4, 126.1, 125.9, 124.3, 124.1, 122.0, 121.8, 119.0, 116.1, 116.0, 110.8, 55.5. HRMS-DART $(\mathrm{m} / \mathrm{z}):[\mathrm{M}+\mathrm{H}]^{+}$calcd for $\mathrm{C}_{23} \mathrm{H}_{18} \mathrm{NOS}$, 356.1104 ; found, 356.1102 .

\section{4-(12H-Benzo[b]phenothiazin-12-yl)benzonitrile (PTH4)}

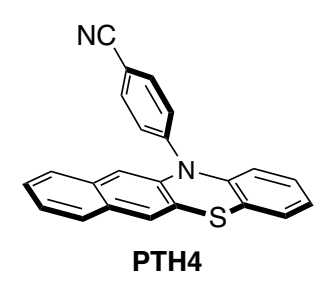

The organophotoredox catalyst PTH4 was purified by flash chromatography on silica gel (100:090:10, hexane/EtOAc). $0.4 \mathrm{mmol}$ scale; $76 \mathrm{mg}, 0.21 \mathrm{mmol}, 54 \%$ isolated yield. White solid. M.p. 217-220 ${ }^{\circ} \mathrm{C}$. IR (neat) 747, 1308, 1448, 1503, 1588, 1605, 2218, $3055 \mathrm{~cm}^{-1} .{ }^{1} \mathbf{H}$ NMR (400 MHz, 
$\left.\mathrm{CDCl}_{3}\right) \delta 7.82(\mathrm{~s}, 1 \mathrm{H}), 7.73-7.60(\mathrm{~m}, 4 \mathrm{H}), 7.45-7.37(\mathrm{~m}, 4 \mathrm{H}), 7.27-7.21(\mathrm{~m}, 3 \mathrm{H}), 7.14(\mathrm{~m}, 1 \mathrm{H}), 7.04$ $(\mathrm{d}, J=8.0 \mathrm{~Hz}, 1 \mathrm{H}) .{ }^{13} \mathbf{C} \mathbf{N M R}\left(100.6 \mathrm{MHz}, \mathrm{CDCl}_{3}\right) \delta 148.2,141.3,139.2,133.9,132.4,131.2,129.6$, $129.4,128.5,127.3$ ( $\times 2 \mathrm{C}), 126.7,126.6,126.4,126.2,125.4,123.6,120.6,120.5,119.1,106.2$. HRMS-DART $(\mathrm{m} / \mathrm{z})$ : $[\mathrm{M}+\mathrm{H}]^{+}$calcd for $\mathrm{C}_{23} \mathrm{H}_{15} \mathrm{~N}_{2} \mathrm{~S}, 351.0950$; found, 351.0946.

\section{- Characterization Data for Redox Active Esters}

1,3-Dioxoisoindolin-2-yl 4-Cyano-2,2-diethylbutanoate (3d)

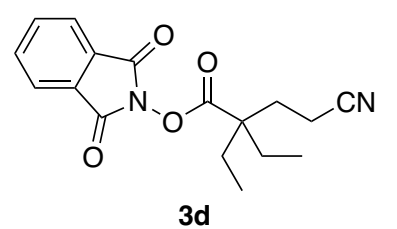

The redox active ester 3d was purified by flash chromatography on silica gel (100:0-90:10, hexane/EtOAc). $6.0 \mathrm{mmol} \mathrm{scale;} 1.41 \mathrm{~g}, 4.48 \mathrm{mmol}, 75 \%$ isolated yield. White solid. M.p. 110$113^{\circ} \mathrm{C} . \mathbf{I R}$ (neat) 696, 877, 1062, 1369, 1467, 1740, 1780, $2972 \mathrm{~cm}^{-1} .{ }^{\mathbf{1}} \mathbf{H} \mathbf{N M R}\left(400 \mathrm{MHz}, \mathrm{CDCl}_{3}\right)$ $\delta 7.91-7.87(\mathrm{~m}, 2 \mathrm{H}), 7.83-7.79(\mathrm{~m}, 2 \mathrm{H}), 2.51-2.47(\mathrm{~m}, 2 \mathrm{H}), 2.18-2.14(\mathrm{~m}, 2 \mathrm{H}), 1.91-1.76(\mathrm{~m}, 4 \mathrm{H})$, $1.00(\mathrm{t}, J=7.6 \mathrm{~Hz}, 6 \mathrm{H}) .{ }^{13} \mathbf{C} \mathbf{N M R}\left(100.6 \mathrm{MHz}, \mathrm{CDCl}_{3}\right) \delta 171.9,161.9,134.8,128.8,123.9,119.3$, 49.2, 29.6, 26.2, 12.3, 7.8. HRMS-DART $(\mathrm{m} / \mathrm{z})$ : $[\mathrm{M}+\mathrm{H}]^{+}$calcd for $\mathrm{C}_{17} \mathrm{H}_{19} \mathrm{~N}_{2} \mathrm{O}_{4}, 315.1339$; found, 315.1345 .

\section{1,3-Dioxoisoindolin-2-yl 1-Methylcyclopentane-1-carboxylate (3e)}

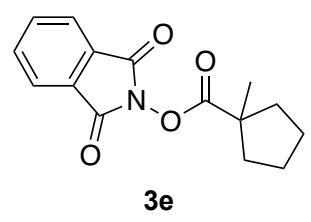

The redox active ester 3e was purified by flash chromatography on silica gel (100:0-90:10, hexane/EtOAc). $10.0 \mathrm{mmol}$ scale; $1.92 \mathrm{~g}, 7.01 \mathrm{mmol}, 70 \%$ isolated yield. White solid. M.p. $32-35{ }^{\circ} \mathrm{C}$. IR (neat) 518, 698, 876, 1013, 1133, 1184, 1357, 1465, $1738 \mathrm{~cm}^{-1} .{ }^{\mathbf{1}} \mathbf{H} \mathbf{~ N M R}\left(400 \mathrm{MHz}, \mathrm{CDCl}_{3}\right) \delta$ 7.89-7.87 (m, 2H), 7.80-7.78 (m, 2H), 2.41-2.34 (m, 2H), 1.84-1.64 (m, 6H), 1.48 (s, 3H). ${ }^{13}$ C NMR $\left(100.6 \mathrm{MHz}, \mathrm{CDCl}_{3}\right) \delta 174.6,162.2,134.6,129.1,123.9,48.3,38.2,25.2,24.6$. HRMS-DART $(m / z):\left[\mathrm{M}+\mathrm{NH}_{4}\right]^{+}$calcd for $\mathrm{C}_{15} \mathrm{H}_{19} \mathrm{~N}_{2} \mathrm{O}_{4}, 291.1339$; found, 291.1330 .

\section{1,3-Dioxoisoindolin-2-yl 4-Methyltetrahydro-2H-pyran-4-carboxylate (3h)}

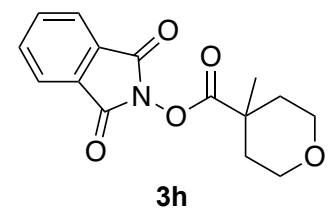

The redox active ester $\mathbf{3 h}$ was purified by flash chromatography on silica gel (100:0-90:10, hexane/EtOAc). $7.8 \mathrm{mmol} \mathrm{scale;} 1.98 \mathrm{~g}, 6.83 \mathrm{mmol}, 88 \%$ isolated yield. White solid. M.p. $83-87{ }^{\circ} \mathrm{C}$. IR (neat) 517, 563, 696, 789, 838, 875, 966, 1035, 1101, 1186, 1353, $1735 \mathrm{~cm}^{-1} .{ }^{1} \mathbf{H}$ NMR (400 MHz, $\left.\mathrm{CDCl}_{3}\right) \delta 7.91-7.89(\mathrm{~m}, 2 \mathrm{H}), 7.81-7.79(\mathrm{~m}, 2 \mathrm{H}), 3.91-3.88(\mathrm{~m}, 2 \mathrm{H}), 3.67(\mathrm{t}, J=11.5 \mathrm{~Hz}, 2 \mathrm{H}) 1.71-$ 
$1.64(\mathrm{~m}, 2 \mathrm{H}), 1.51(\mathrm{~s}, 3 \mathrm{H}) .{ }^{13} \mathbf{C} \mathbf{N M R}\left(100.6 \mathrm{MHz}, \mathrm{CDCl}_{3}\right) \delta 172.9,162.0,134.8,129.0,124.0,65.0$, 41.0, 35.4, 26.5. HRMS-DART $(\mathrm{m} / \mathrm{z})$ : $\left[\mathrm{M}+\mathrm{NH}_{4}\right]^{+}$calcd for $\mathrm{C}_{15} \mathrm{H}_{19} \mathrm{~N}_{2} \mathrm{O}_{5}, 307.1288$; found, 307.1286.

\section{- Procedure for Organophotoredox Catalyzed Decarboxylative Alkoxyalkylation of Alkene n}

The reaction in Table 1, entry 11 is representative. In a glovebox, to an oven-dried vial with a stirring bar was added redox active ester $\mathbf{3 a}(49.5 \mathrm{mg}, 0.2 \mathrm{mmol})$, styrene $\mathbf{2 a}(22.9 \mu \mathrm{l}, 0.2 \mathrm{mmol})$ and alcohol 1a $(54.4 \mu \mathrm{l}, 0.6 \mathrm{mmol})$ and $\mathrm{MeCN}(380 \mu \mathrm{l})$. Then, $200 \mu \mathrm{l}$ of stock solution of PTH1/MeCN $(0.79 \mathrm{mg}, 0.002 \mathrm{mmol} / 200 \mu \mathrm{l})$ and $20 \mu \mathrm{l}$ of stock solution of $\mathrm{LiBF}_{4} / \mathrm{MeCN}(0.19 \mathrm{mg}, 0.002 \mathrm{mmol} /$ $20 \mu \mathrm{l})$ were added to the reaction mixture. After sealing the vial with a cap and removed from the glove box, the reaction was stirred and irradiated with a $34 \mathrm{~W}$ blue LED ( $0.5 \mathrm{~cm}$ away) with a cooling fan to keep the temperature around $40{ }^{\circ} \mathrm{C}$ (Figure S1). After $2 \mathrm{~h}$, the reaction was quenched with a short plug of silica gel using diethyl ether. After volatiles were removed under reduced pressure, purification by flash column chromatography (hexane) on silica gel gave 4aaa (29.6 mg, 60\%) as a colorless oil.

The reaction conditions for scope of alcohol (Scheme 2, middle). In a glovebox, to an ovendried vial with a stirring bar was added PTH1 (3.9 mg, $0.01 \mathrm{mmol})$, LiBF (0.9 mg, $0.01 \mathrm{mmol}$ ), redox active ester 3a $(49.5 \mathrm{mg}, 0.2 \mathrm{mmol})$, styrene $\mathbf{2 a}(22.9 \mu \mathrm{l}, 0.2 \mathrm{mmol})$ and alcohol $\mathbf{1}(0.6 \mathrm{mmol})$ in $\mathrm{MeCN}(600 \mu \mathrm{l})$. After sealing the vial with a cap and removed from the glove box, the reaction was stirred and irradiated with a $34 \mathrm{~W}$ blue LED ( $0.5 \mathrm{~cm}$ away) with a cooling fan to keep the temperature around $40{ }^{\circ} \mathrm{C}$ (Figure S1). After $6 \mathrm{~h}$, the reaction was quenched with a short plug of silica gel using diethyl ether. After volatiles were removed under reduced pressure, purification by flash column chromatography on silica gel gave the coupling product.

1 mmol scale. In a glovebox, to an oven-dried vial with a stirring bar was added redox active ester 3a (247.3 mg, $1.0 \mathrm{mmol})$, styrene $2 \mathbf{a}(115 \mu \mathrm{l}, 1.0 \mathrm{mmol})$, PTH1 (1.9 mg, $0.01 \mathrm{mmol})$ and alcohol 1a ( $272 \mu \mathrm{l}, 0.6 \mathrm{mmol})$ and $\mathrm{MeCN}(2.9 \mathrm{ml})$. Then, $100 \mu \mathrm{l}$ of stock solution of $\mathrm{LiBF}_{4} / \mathrm{MeCN}(0.9 \mathrm{mg}, 0.02$ $\mathrm{mmol} / 200 \mu \mathrm{l})$ were added to the reaction mixture. After sealing the vial with a cap and removed from the glove box, the reaction was stirred and irradiated with a $34 \mathrm{~W}$ blue LED ( $0.5 \mathrm{~cm}$ away) with a cooling fan to keep the temperature around $40{ }^{\circ} \mathrm{C}$ (Figure S1). After $2 \mathrm{~h}$, the reaction was quenched with a short plug of silica gel using diethyl ether. After volatiles were removed under reduced pressure, purification by flash column chromatography (hexane) on silica gel gave 4aaa (139.2 mg, 56\%) as a colorless oil.

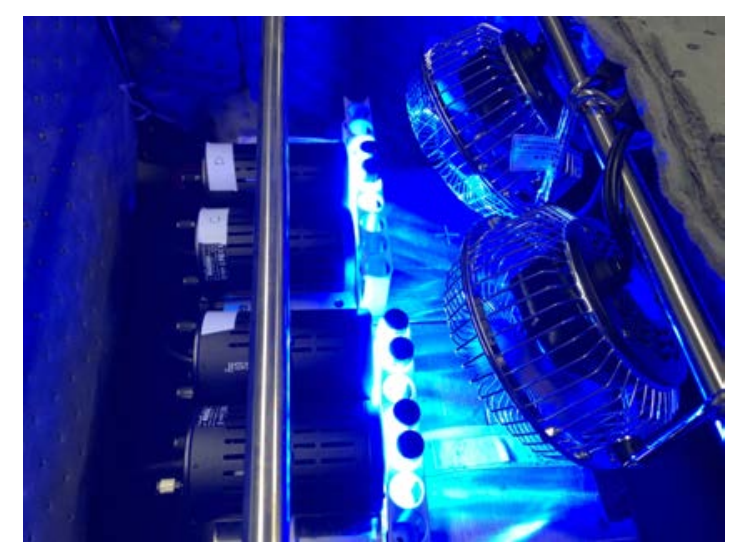

Figure S1. Light set up 


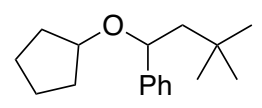

4aaa

The product 4aaa was purified by flash chromatography on silica gel (hexane) (Table 1, entry 1; $29.6 \mathrm{mg}, 0.12 \mathrm{mmol}, 60 \%$ isolated yield). Colorless oil. IR (neat) 699, 736, 1090, 1363, 1452, 2869, 2902, $2951 \mathrm{~cm}^{-1} .{ }^{1} \mathbf{H}$ NMR $\left(600 \mathrm{MHz}, \mathrm{CDCl}_{3}\right) \delta 7.33-7.22(\mathrm{~m}, 5 \mathrm{H}), 4.42(\mathrm{dd}, J=9.0,3.0 \mathrm{~Hz}, 1 \mathrm{H})$, 3.71 (quin, $J=4.9 \mathrm{~Hz}, 1 \mathrm{H}), 1.77-1.37(\mathrm{~m}, 10 \mathrm{H}), 0.97(\mathrm{~s}, 9 \mathrm{H}) .{ }^{13} \mathbf{C ~ N M R}\left(150.9 \mathrm{MHz}, \mathrm{CDCl}_{3}\right) \delta 145.2$, 128.2, 126.9, 126.6, 78.5, 77.7, 52.5, 33.3, 31.1, 30.6, 30.2, 23.3, 23.3. HRMS-DART $(\mathrm{m} / z)$ : $\left[\mathrm{M}+\mathrm{NH}_{4}\right]^{+}$calcd for $\mathrm{C}_{17} \mathrm{H}_{30} \mathrm{NO}, 264.2322$; found, 264.2336 .

\section{4-(Cyclopentyloxy)-2,2-dimethyl-4-phenylbutyl Acetate (4aab)}

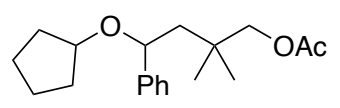

$4 a a b$

The product 4aab was purified by flash chromatography on silica gel (100:0-80:20, hexane/EtOAc) (Scheme 2; $40.8 \mathrm{mg}, 0.13 \mathrm{mmol}, 67 \%$ isolated yield). Colorless oil. IR (neat) 701, 1034, 1239, 1375, 1453, 1736, 2872, $2957 \mathrm{~cm}^{-1} .{ }^{1} \mathbf{H}$ NMR (400 MHz, $\left.\mathrm{CDCl}_{3}\right) \delta 7.35-7.23(\mathrm{~m}, 5 \mathrm{H})$, $4.43(\mathrm{~d}, J=9.2 \mathrm{~Hz}, 1 \mathrm{H}), 3.95-3.84(\mathrm{~m}, 2 \mathrm{H}), 3.69$ (quin, $J=4.5 \mathrm{~Hz}, 1 \mathrm{H}), 2.05$ (s, 3H), 1.84-1.37 (m, $10 \mathrm{H}), 1.02(\mathrm{~s}, 3 \mathrm{H}), 0.99(\mathrm{~s}, 3 \mathrm{H}) .{ }^{13} \mathbf{C} \mathbf{N M R}\left(100.6 \mathrm{MHz}, \mathrm{CDCl}_{3}\right) \delta 171.2,144.5,128.3,127.2,126.5$, 78.6, 77.0, 72.8, 47.8, 33.9, 33.3, 31.1, 25.3, 24.9, 23.3, 23.3, 20.9. HRMS-ESI $(\mathrm{m} / \mathrm{z}):[\mathrm{M}+\mathrm{K}]^{+}$calcd for $\mathrm{C}_{19} \mathrm{H}_{28} \mathrm{KO}_{3}, 343.1670$; found, 343.1671.

\section{[4-(Benzyloxy)-1-(cyclopentyloxy)-3,3-dimethylbutyl]benzene (4aac)}

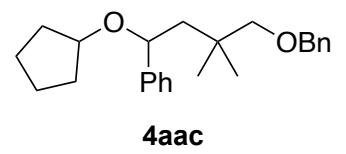

The product 4aac was purified by flash chromatography on silica gel (100:0-90:10, hexane/EtOAc) (Scheme 2; $42.7 \mathrm{mg}, 0.12 \mathrm{mmol}, 61 \%$ isolated yield). Colorless oil. IR (neat) 699, 743, 1094, 1361, 1453, 2869, $2954 \mathrm{~cm}^{-1} .{ }^{1} \mathbf{H}$ NMR (400 MHz, $\left.\mathrm{CDCl}_{3}\right) \delta 7.34-7.21$ (m, 10H), 4.54$4.41(\mathrm{~m}, 3 \mathrm{H}), 3.67$ (quin, $J=4.4 \mathrm{~Hz}, 1 \mathrm{H}), 3.27-3.17(\mathrm{~m}, 2 \mathrm{H}), 1.80-1.38(\mathrm{~m}, 10 \mathrm{H}), 1.01(\mathrm{~s}, 3 \mathrm{H}), 1.00$ $(\mathrm{s}, 3 \mathrm{H}) .{ }^{13} \mathbf{C} \mathbf{N M R}\left(100.6 \mathrm{MHz}, \mathrm{CDCl}_{3}\right) \delta 145.0,139.1,128.2,128.2,127.4,127.2,126.9,126.6,79.6$, 78.5, 77.3, 73.2, 48.0, 34.9, 33.3, 31.1, 25.5, 25.4, 23.3. HRMS-ESI $(\mathrm{m} / \mathrm{z}):[\mathrm{M}+\mathrm{Na}]^{+}$calcd for $\mathrm{C}_{24} \mathrm{H}_{32} \mathrm{NaO}_{2}, 375.2295$; found, 375.2292.

6-(Cyclopentyloxy)-4,4-diethyl-6-phenylhexanenitrile (4aad)

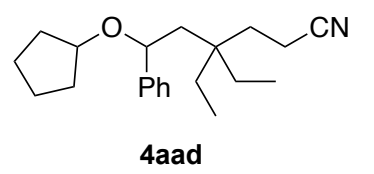


The product 4aad was purified by flash chromatography on silica gel (100:0-80:20, hexane/EtOAc) (Scheme 2; $27.0 \mathrm{mg}, 0.09 \mathrm{mmol}, 43 \%$ isolated yield). Colorless oil. IR (neat) 700, 756, 1063, 1341, 1453, 2874, $2961 \mathrm{~cm}^{-1} .{ }^{1} \mathbf{H}$ NMR (400 MHz, $\left.\mathrm{CDCl}_{3}\right) \delta 7.36-7.24(\mathrm{~m}, 5 \mathrm{H}), 4.37$ (dd, $J=2 ., 9.6 \mathrm{~Hz}, 1 \mathrm{H}), 3.68(\mathrm{~m}, 1 \mathrm{H}), 2.56(\mathrm{~m}, 1 \mathrm{H}), 2.15(\mathrm{~m}, 1 \mathrm{H}), 1.87(\mathrm{~m}, 1 \mathrm{H}), 1.74-1.11(\mathrm{~m}, 15 \mathrm{H})$, $0.81(\mathrm{t}, J=7.4 \mathrm{~Hz}, 3 \mathrm{H}), 0.76(\mathrm{t}, J=7.6 \mathrm{~Hz}, 3 \mathrm{H}) .{ }^{13} \mathbf{C} \mathbf{N M R}\left(100.6 \mathrm{MHz}, \mathrm{CDCl}_{3}\right) \delta 144.6,128.4$, 127.3, 126.2, 120.9, 78.6, 76.3, 43.7, 37.9, 33.4, 32.1, 31.0, 27.8, 27.6, 23.4, 23.3, 11.8, 7.4, 7.1. HRMS-ESI $(\mathrm{m} / \mathrm{z}):[\mathrm{M}+\mathrm{K}]^{+}$calcd for $\mathrm{C}_{21} \mathrm{H}_{31} \mathrm{KNO}, 352.2037$; found, 352.2038.

\section{[1-(Cyclopentyloxy)-2-(1-methylcyclopentyl)ethyl]benzene (4aae)}

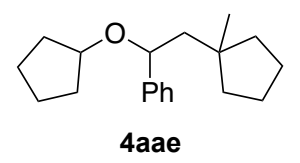

The product 4aae was purified by flash chromatography on silica gel (100:0, hexane/EtOAc) (Scheme 2; $31.6 \mathrm{mg}, 0.12 \mathrm{mmol}, 58 \%$ isolated yield). Pale yellow oil. IR (neat) 700, 762, 1088, 1324, 1451, 2869, $2951 \mathrm{~cm}^{-1} .{ }^{1} \mathbf{H}$ NMR $\left(400 \mathrm{MHz}, \mathrm{CDCl}_{3}\right) \delta 7.35-7.21(\mathrm{~m}, 5 \mathrm{H}), 4.42(\mathrm{dd}, J=3.3,8.8 \mathrm{~Hz}$, $1 \mathrm{H}), 3.71$ (quin, $J=4.8 \mathrm{~Hz}, 1 \mathrm{H}), 1.86-1.25(\mathrm{~m}, 18 \mathrm{H}), 1.03(\mathrm{~s}, 3 \mathrm{H}) .{ }^{13} \mathbf{C} \mathbf{N M R}\left(100.6 \mathrm{MHz}, \mathrm{CDCl}_{3}\right)$ $\delta 145.2,128.2,126.9,126.6,78.5,78.1,51.1,42.0,40.2,40.1,33.3,31.2,26.1,24.3,23.9,23.4,23.3$. HRMS-DART (m/z): [M+NH$]^{+}$calcd for $\mathrm{C}_{19} \mathrm{H}_{32} \mathrm{NO}, 290.2478$; found, 290.2483.

\section{[1-(Cyclopentyloxy)-2-(1-methylcyclohexyl)ethyl]benzene (4aaf)}

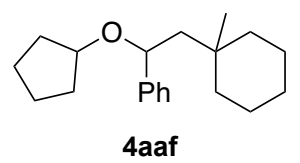

The product 4aaf was purified by flash chromatography on silica gel (100:0-95:5, hexane/EtOAc) (Scheme 2; $41.8 \mathrm{mg}, 0.15 \mathrm{mmol}, 73 \%$ isolated yield). Pale yellow oil. IR (neat) 699, 763, 1068, 1341, 1451, 2863, $2922 \mathrm{~cm}^{-1} .{ }^{1} \mathbf{H}$ NMR $\left(400 \mathrm{MHz}, \mathrm{CDCl}_{3}\right) \delta 7.34-7.21(\mathrm{~m}, 5 \mathrm{H}), 4.45(\mathrm{dd}, J=2.8,8.7 \mathrm{~Hz}$, $1 \mathrm{H}), 3.69$ (quin, $J=4.8 \mathrm{~Hz}, 1 \mathrm{H}), 1.82-1.19(\mathrm{~m}, 20 \mathrm{H}), 0.98(\mathrm{~s}, 3 \mathrm{H}) .{ }^{13} \mathbf{C} \mathbf{N M R}\left(100.6 \mathrm{MHz}, \mathrm{CDCl}_{3}\right)$ $\delta 145.5,128.2,126.9,126.6,78.6,38.6,38.4,33.3,31.1,26.5,23.3,23.3,22.1,22.0$. HRMS-DART $(\mathrm{m} / \mathrm{z}):\left[\mathrm{M}+\mathrm{NH}_{4}\right]^{+}$calcd for $\mathrm{C}_{20} \mathrm{H}_{34} \mathrm{NO}, 304.2635$; found, 304.2619 .

\section{tert-Butyl 4-[2-(Cyclopentyloxy)-2-phenylethyl]-4-methylpiperidine-1-carboxylate (4aag)}

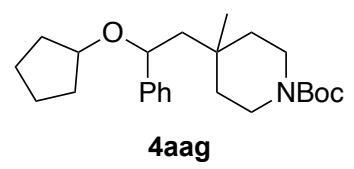

The product 4aag was purified by flash chromatography on silica gel (98:2-80:20, hexane/EtOAc) (Scheme 2; $33.3 \mathrm{mg}, 0.09 \mathrm{mmol}, 43 \%$ isolated yield). Colorless oil. IR (neat) 700, 766, 1090, 1160, 1245, 1420, 1689, $2957 \mathrm{~cm}^{-1} .{ }^{1} \mathbf{H}$ NMR (400 MHz, $\left.\mathrm{CDCl}_{3}\right) \delta 7.35-7.23(\mathrm{~m}, 5 \mathrm{H}), 4.46(\mathrm{~d}, J=8.8 \mathrm{~Hz}$, $1 \mathrm{H}$ ), 3.69 (quin, $J=4.5 \mathrm{~Hz}, 1 \mathrm{H}), 3.53-3.42(\mathrm{~m}, 2 \mathrm{H}), 3.31-3.25(\mathrm{~m}, 2 \mathrm{H}), 1.88(\mathrm{~m}, 1 \mathrm{H}), 1.71-1.26(\mathrm{~m}$, 22H), 1.06 (s, 3H). ${ }^{13} \mathbf{C}$ NMR (100.6 MHz, $\left.\mathrm{CDCl}_{3}\right) \delta 155.0,144.8,128.3,127.1,126.5,79.1,78.5$, 76.7, 49.8, 37.6, 33.3, 31.7, 31.1, 28.5, 24.7, 23.3. HRMS-DART $(\mathrm{m} / z):[\mathrm{M}+\mathrm{H}]^{+}$calcd for $\mathrm{C}_{24} \mathrm{H}_{38} \mathrm{NO}_{3}, 388.2846$; found, 388.2851. 


\section{4-[2-(Cyclopentyloxy)-2-phenylethyl]-4-methyltetrahydro-2H-pyran (4aah)}

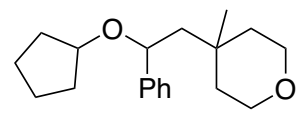

4aah

The product 4aah was purified by flash chromatography on silica gel (100:0-80:20, hexane/EtOAc) (Scheme 2; $30.0 \mathrm{mg}, 0.10 \mathrm{mmol}, 52 \%$ isolated yield). Colorless oil. IR (neat) 700, 1017, 1109, 1166, 1343, 1453, 2867, $2953 \mathrm{~cm}^{-1} .{ }^{1} \mathbf{H}$ NMR $\left(600 \mathrm{MHz}, \mathrm{CDCl}_{3}\right) \delta 7.34-7.24$ (m, 5H), $4.48(\mathrm{dd}, J=9.0,3.0 \mathrm{~Hz}, 1 \mathrm{H}), 3.75-3.61(\mathrm{~m}, 5 \mathrm{H}), 1.92(\mathrm{~m}, 1 \mathrm{H}), 1.72-1.40(\mathrm{~m}, 12 \mathrm{H}), 1.32(\mathrm{~m}, 1 \mathrm{H})$, 1.11 (s, 3H). ${ }^{13} \mathbf{C}$ NMR (100.6 MHz, $\left.\mathrm{CDCl}_{3}\right) \delta 144.8,128.3,127.1,126.5,78.5,76.7,64.0,63.8,50.6$, $38.5,38.3,33.3,31.1,30.9,24.8,23.3(\times 2 \mathrm{C})$. HRMS-DART $(\mathrm{m} / z):[\mathrm{M}+\mathrm{H}]^{+}$calcd for $\mathrm{C}_{19} \mathrm{H}_{29} \mathrm{O}_{2}$, 289.2162; found, 289.2147.

\section{(1s,3s)-1-[2-(Cyclopentyloxy)-2-phenylethyl]adamantane (4aai)}

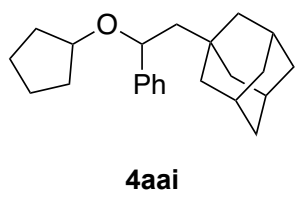

The product 4aai was purified by flash chromatography on silica gel (100:0-90:10, hexane/EtOAc) (Scheme 2; $38.3 \mathrm{mg}, 0.12 \mathrm{mmol}, 59 \%$ isolated yield). Pale yellow oil. IR (neat) 699, 760, 1085, 1168, 1347, 1450, 2845, $2896 \mathrm{~cm}^{-1} .{ }^{1} \mathbf{H}$ NMR $\left(400 \mathrm{MHz}, \mathrm{CDCl}_{3}\right) \delta 7.33-7.21(\mathrm{~m}, 5 \mathrm{H}), 4.48(\mathrm{dd}, J=9.2$, $2.4 \mathrm{~Hz}, 1 \mathrm{H}), 3.70$ (quin, $J=4.8 \mathrm{~Hz}, 1 \mathrm{H}), 1.94(\mathrm{~s}, 3 \mathrm{H}), 1.78-1.40(\mathrm{~m}, 21 \mathrm{H}), 1.24(\mathrm{~m}, 1 \mathrm{H}) .{ }^{13} \mathbf{C} \mathbf{N M R}$ $\left(100.6 \mathrm{MHz}, \mathrm{CDCl}_{3}\right) \delta 145.3,128.2,126.9,126.6,78.5,76.2,53.5,43.1,37.2,33.3,32.7,31.1,28.8$, 23.3, 23.3. HRMS-DART $(\mathrm{m} / \mathrm{z}):\left[\mathrm{M}+\mathrm{NH}_{4}\right]^{+}$calcd for $\mathrm{C}_{23} \mathrm{H}_{36} \mathrm{NO}, 342.2791$; found, 342.2796.

\section{[2-Cyclohexyl-1-(cyclopentyloxy)ethyl]benzene (4aaj)}

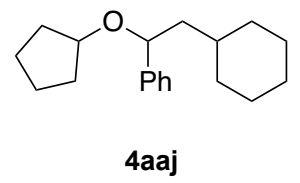

The reaction conditions were modified. The reaction was carried out with $\mathbf{1 a}(54.4 \mu \mathrm{l}, 0.6 \mathrm{mmol}$ $0.6 \mathrm{mmol}), \mathbf{2 a}(22.9 \mu \mathrm{l}, 0.2 \mathrm{mmol}), 3 \mathbf{j}$ (54.7 mg, $0.2 \mathrm{mmol})$, PTH1 (3.9 mg, $0.01 \mathrm{mmol}, 5 \mathrm{~mol} \%$ ), and $\mathrm{LiBF}_{4}(0.9 \mathrm{mg}, 0.01 \mathrm{mmol}, 5 \mathrm{~mol} \%)$ in $\mathrm{MeCN}(600 \mu \mathrm{L})$ under blue LED irradiation for $24 \mathrm{~h}$. The product 4aaj was purified by flash chromatography on silica gel (100:0-95:5, hexane/EtOAc) (Scheme 2; $31.7 \mathrm{mg}, 0.12 \mathrm{mmol}, 58 \%$ isolated yield). Yellow oil. IR (neat) 700, 754, 1067, 1324, 1448, 2850, $2920 \mathrm{~cm}^{-1} .{ }^{1} \mathbf{H}$ NMR $\left(400 \mathrm{MHz}, \mathrm{CDCl}_{3}\right) \delta 7.34-7.22(\mathrm{~m}, 5 \mathrm{H}), 4.37(\mathrm{dd}, J=9.6,3.6 \mathrm{~Hz}$, $1 \mathrm{H}), 3.75$ (quin, $J=4.7 \mathrm{~Hz}, 1 \mathrm{H}), 1.82-0.85(\mathrm{~m}, 21 \mathrm{H}) .{ }^{13} \mathbf{C}$ NMR $\left(100.6 \mathrm{MHz}, \mathrm{CDCl}_{3}\right) \delta 144.4,128.2$, 127.0, 126.6, 78.6, 77.1, 46.8, 34.1, 34.0, 33.1, 32.9, 31.5, 26.7, 26.3, 26.2, 23.3 (× 2C). HRMSDART $(\mathrm{m} / z)$ : $\left[\mathrm{M}+\mathrm{NH}_{4}\right]^{+}$calcd for $\mathrm{C}_{19} \mathrm{H}_{32} \mathrm{NO}, 290.2478$; found, 290.2496. 


\section{4-[2-(Cyclopentyloxy)-2-phenylethyl]etrahydro-2H-pyran (4aak)}

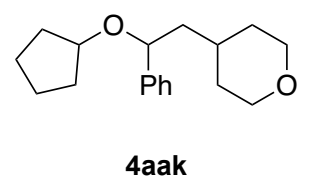

The reaction conditions were modified. The reaction was carried out with 1a $(54.4 \mu 1,0.6 \mathrm{mmol}$ $0.6 \mathrm{mmol}$ ), 2a (22.9 $\mu \mathrm{l}, 0.2 \mathrm{mmol}), 3 \mathbf{k}(55.1 \mathrm{mg}, 0.2 \mathrm{mmol})$, PTH1 (3.9 mg, $0.01 \mathrm{mmol}, 5 \mathrm{~mol} \%$ ), and $\mathrm{LiBF}_{4}(0.9 \mathrm{mg}, 0.01 \mathrm{mmol}, 5 \mathrm{~mol} \%)$ in $\mathrm{MeCN}(10 \mu \mathrm{l})$ and DCM $(590 \mu \mathrm{L})$ under blue LED irradiation for $12 \mathrm{~h}$. The product 4aak was purified by flash chromatography on silica gel (100:0 80:20, hexane/EtOAc) (Scheme 2; $19.8 \mathrm{mg}, 0.07 \mathrm{mmol}, 36 \%$ isolated yield). Colorless oil. IR (neat) 700, 757, 1014, 1093, 1138, 2837, $2967 \mathrm{~cm}^{-1} .{ }^{1} \mathbf{H}$ NMR (400 MHz, CDCl $)_{3} \delta 7.36-7.24(\mathrm{~m}, 5 \mathrm{H})$, $4.38(\mathrm{~m}, 1 \mathrm{H}), 3.98-3.91(\mathrm{~m}, 2 \mathrm{H}), 3.75$ (quin, $J=4.8 \mathrm{~Hz}, 1 \mathrm{H}), 3.37$ (tt, $J=12.0,1.6 \mathrm{~Hz}, 2 \mathrm{H}), 1.77-$ $1.26(\mathrm{~m}, 15 \mathrm{H}) .{ }^{13} \mathrm{C}$ NMR $\left(100.6 \mathrm{MHz}, \mathrm{CDCl}_{3}\right) \delta 143.9,128.3,127.2,126.5,78.5,76.6,68.1,68.0$, 46.2, 33.7, 33.1, 32.7, 31.6, 31.5, 23.3, 23.2. HRMS-DART $(\mathrm{m} / \mathrm{z}):[\mathrm{M}+\mathrm{H}]^{+}$calcd for $\mathrm{C}_{18} \mathrm{H}_{27} \mathrm{O}_{2}$, 275.2006; found, 275.2006.

tert-Butyl 4-[2-(Cyclopentyloxy)-2-phenylethyl]piperidine-1-carboxylate (4aal)

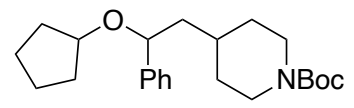

4aal

The reaction conditions were modified. The reaction was carried out with 1a ( $54.4 \mu 1,0.6 \mathrm{mmol}$ $0.6 \mathrm{mmol}$ ), $2 \mathrm{a}$ (22.9 $\mu \mathrm{l}, 0.2 \mathrm{mmol}), 31$ (74.9 mg, $0.2 \mathrm{mmol})$, PTH1 (3.9 mg, $0.01 \mathrm{mmol}, 5 \mathrm{~mol} \%$ ), and $\mathrm{LiBF}_{4}(0.9 \mathrm{mg}, 0.01 \mathrm{mmol}, 5 \mathrm{~mol} \%)$ in $\mathrm{MeCN}(10 \mu \mathrm{l})$ and DCM $(590 \mu \mathrm{L})$ under blue LED irradiation for $12 \mathrm{~h}$. The product 4aal was purified by flash chromatography on silica gel (100:0-80:20, hexane/EtOAc) (Scheme 2; $36.6 \mathrm{mg}, 0.10 \mathrm{mmol}, 49 \%$ isolated yield). Colorless oil. IR (neat) 1085, 1168, 1244, 1422, 1692, 2868, $2928 \mathrm{~cm}^{-1} .{ }^{1} \mathbf{H}$ NMR (400 MHz, $\left.\mathrm{CDCl}_{3}\right) \delta 7.35-7.25(\mathrm{~m}, 5 \mathrm{H}), 4.37$ (dd, $J=9.2,4.0 \mathrm{~Hz}, 1 \mathrm{H}$ ), 4.06 (br s, 2H), 3.75 (quin, $J=4.8 \mathrm{~Hz}, 1 \mathrm{H}$ ), 2.67 (br s, 2H), 1.75-1.37 (m, 22H), 1.13-1.10 (m, 2H). ${ }^{13} \mathbf{C}$ NMR (100.6 MHz, $\left.\mathrm{CDCl}_{3}\right) \delta 154.9,143.8,128.3,127.2,126.5,79.1$, 78.6, 76.9, 45.8, 44.0, 33.1 ( $\times 2 \mathrm{C}), 32.7,32.6,31.7,31.5,28.4,23.3,23.3$. HRMS-ESI $(\mathrm{m} / z)$ : $[\mathrm{M}+\mathrm{Na}]^{+}$calcd for $\mathrm{C}_{23} \mathrm{H}_{35} \mathrm{NNaO}_{3}, 396.2509$; found, 396.2514.

\section{Methyl 3-(3,3-Dimethyl-1-phenylbutoxy)-2,2-dimethylpropanoate (4baa)}

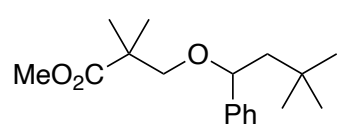

4 baa

The product 4baa was purified by flash chromatography on silica gel (98:2-80:20, hexane/EtOAc) (Scheme 2; $30.4 \mathrm{mg}, 0.10 \mathrm{mmol}, 52 \%$ isolated yield). Pale yellow oil. IR (neat) 701, 1100, 1149, 1228, 1475, 1736, $2950 \mathrm{~cm}^{-1}$. ${ }^{\mathbf{1}} \mathbf{H}$ NMR (400 MHz, $\left.\mathrm{CDCl}_{3}\right) \delta 7.34-7.30(\mathrm{~m}, 2 \mathrm{H}), 7.24-7.22(\mathrm{~m}, 3 \mathrm{H})$, $4.27(\mathrm{~d}, J=9.6 \mathrm{~Hz}, 1 \mathrm{H}), 3.64(\mathrm{~s}, 3 \mathrm{H}), 3.29$ (d, $J=8.4 \mathrm{~Hz}, 1 \mathrm{H}), 3.14(\mathrm{~d}, J=8.4 \mathrm{~Hz}, 1 \mathrm{H}), 1.74(\mathrm{dd}, J$ $=14.4,9.6 \mathrm{~Hz}, 1 \mathrm{H}), 1.33(\mathrm{~d}, J=14.4 \mathrm{~Hz}, 1 \mathrm{H}), 1.18(\mathrm{~s}, 3 \mathrm{H}), 1.13(\mathrm{~s}, 3 \mathrm{H}), 0.95(\mathrm{~s}, 9 \mathrm{H}) .{ }^{13} \mathbf{C}$ NMR 
$\left(100.6 \mathrm{MHz} \mathrm{CDCl}_{3}\right) \delta 177.1,144.2,128.3,127.1,126.3,80.5,75.6,52.4,51.6,43.5,30.5,30.1,22.8$, 22.3. HRMS-DART $(\mathrm{m} / \mathrm{z})$ : $\left[\mathrm{M}+\mathrm{NH}_{4}\right]^{+}$calcd for $\mathrm{C}_{18} \mathrm{H}_{32} \mathrm{NO}_{3}, 310.2377$; found, 310.2362.

\section{[1-(But-3-yn-1-yloxy)-3,3-dimethylbutyl]benzene (4caa)}

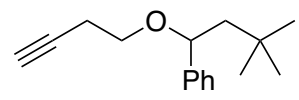

4caa

The product 4caa was purified by flash chromatography on silica gel (99:1-90:10, hexane/EtOAc) (Scheme 2; $33.2 \mathrm{mg}, 0.14 \mathrm{mmol}, 72 \%$ isolated yield). Colorless oil. IR (neat) 635, 700, 1103, 1364, 1453, 2866, 2952, $3310 \mathrm{~cm}^{-1} .{ }^{1} \mathbf{H}$ NMR (400 MHz, $\left.\mathrm{CDCl}_{3}\right) \delta 7.34-7.23(\mathrm{~m}, 5 \mathrm{H}), 4.35(\mathrm{dd}, J=8.8$, $2.8 \mathrm{~Hz}, 1 \mathrm{H}), 3.42-3.32(\mathrm{~m}, 2 \mathrm{H}), 2.48-2.34(\mathrm{~m}, 2 \mathrm{H}), 1.93(\mathrm{~m}, 1 \mathrm{H}), 1.81(\mathrm{dd}, J=14.4,8.8 \mathrm{~Hz}, 1 \mathrm{H})$, $1.42(\mathrm{dd}, J=14.4,2.8 \mathrm{~Hz}, 1 \mathrm{H}), 0.98(\mathrm{~s}, 9 \mathrm{H}) .{ }^{13} \mathbf{C}$ NMR $\left(100.6 \mathrm{MHz}, \mathrm{CDCl}_{3}\right) \delta 144.0,128.4,127.3$, 126.4, 81.6, 80.5, 69.1, 66.7, 52.1, 30.6, 30.2, 20.0. HRMS-DART $(\mathrm{m} / \mathrm{z})$ : $[\mathrm{M}+\mathrm{H}]^{+}$calcd for $\mathrm{C}_{16} \mathrm{H}_{23} \mathrm{O}$, 231.1743 ; found, 231.1745 .

\section{[1-(2-Ethoxyethoxy)-3,3-dimethylbutyl]benzene (4daa)}

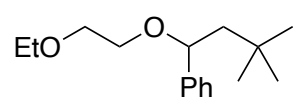

4daa

The reaction conditions were modified. The reaction was carried out with $\mathbf{1 d}$ (19.4 $\mu 1,0.2 \mathrm{mmol}$ ), 2a (45.8 $\mu \mathrm{l}, 0.4 \mathrm{mmol}), 3 \mathbf{3}(98.9 \mathrm{mg}, 0.4 \mathrm{mmol})$, PTH1 (0.79 mg, $0.002 \mathrm{mmol})$, and $\mathrm{LiBF}_{4}(0.19 \mathrm{mg}$, $0.002 \mathrm{mmol})$ in $\mathrm{MeCN}(600 \mu \mathrm{L})$ under blue LED irradiation for $12 \mathrm{~h}$. The product 4daa was purified by flash chromatography on silica gel (100:0-90:10, hexane/EtOAc) (Scheme 2; $35.6 \mathrm{mg}, 0.14 \mathrm{mmol}$, $71 \%$ isolated yield). Pale yellow oil. IR (neat) 700, 737, 1103, 1364, 1452, 2865, $2951 \mathrm{~cm}^{-1} .{ }^{1} \mathbf{H}$ NMR (400 MHz, $\left.\mathrm{CDCl}_{3}\right) \delta 7.34-7.22(\mathrm{~m}, 5 \mathrm{H}), 4.37(\mathrm{dd}, J=8.8,3.2 \mathrm{~Hz}, 1 \mathrm{H}), 3.55-3.36(\mathrm{~m}, 6 \mathrm{H})$, $1.83(\mathrm{dd}, J=14.4,8.8 \mathrm{~Hz}, 1 \mathrm{H}), 1.43(\mathrm{dd}, J=14.4,3.2 \mathrm{~Hz}, 1 \mathrm{H}), 1.18(\mathrm{t}, J=7.0 \mathrm{~Hz}, 3 \mathrm{H}), 0.97$ (s, 9H). ${ }^{13}$ C NMR (100.6 MHz, $\left.\mathrm{CDCl}_{3}\right) \delta 144.2,128.3,127.1,126.5,80.6,69.9,67.8,66.4,52.1,30.6,30.1$, 15.1. HRMS-DART $(\mathrm{m} / \mathrm{z}):[\mathrm{M}+\mathrm{H}]^{+}$calcd for $\mathrm{C}_{16} \mathrm{H}_{27} \mathrm{O}_{2}, 251.2006$; found, 251.2010.

tert-Butyl (2S)-2-[(3,3-Dimethyl-1-phenylbutoxy)methyl]pyrrolidine-1-carboxylate (4eaa)

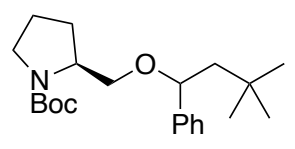

4eaa

The product 4eaa was purified by flash chromatography on silica gel (98:2-95:5, hexane/EtOAc) (Scheme 2; $36.2 \mathrm{mg}, 0.10 \mathrm{mmol}, 50 \%$ isolated yield). Colorless oil. IR (neat) 700, 772, 1102, 1167, 1389, 1693, 2870, $2952 \mathrm{~cm}^{-1}$. Signals for two rotamers and diastereomers were given: ${ }^{\mathbf{1}} \mathbf{H}$ NMR (400 $\left.\mathrm{MHz} \mathrm{CDCl}_{3}\right) \delta 7.33-7.23(\mathrm{~m}, 5 \mathrm{H}), 4.34-4.24(\mathrm{~m}, 1 \mathrm{H}), 3.98-3.08(\mathrm{~m}, 5 \mathrm{H}), 2.02-1.72(\mathrm{~m}, 5 \mathrm{H}), 1.47-$ $1.30(\mathrm{~m}, 10 \mathrm{H}), 0.97$ (br s, 9H). Signals for two rotamers and diasteromers were given: ${ }^{13} \mathbf{C}$ NMR $\left(100.6 \mathrm{MHz}, \mathrm{CDCl}_{3}\right) \delta 154.4,144.4,144.3,128.3,128.3,127.1,126.2,126.1,80.5,80.3,80.2,79.0$, $78.8,69.7,69.5,69.2,68.6,56.9,56.6,56.5,52.5,52.3,52.2,47.1,46.7,46.3,46.3,30.5,30.5,30.2$, 
$30.0,29.9,29.2,29.0,28.7,28.4,28.3,28.0,27.5,24.2,23.6,22.9,22.8$ (only observed peaks). HRMS-DART $(\mathrm{m} / \mathrm{z}):[\mathrm{M}+\mathrm{H}]^{+}$calcd for $\mathrm{C}_{22} \mathrm{H}_{36} \mathrm{NO}_{3}, 362.2690$; found, 362.2688. [ $\left.\alpha\right]_{\mathrm{D}}{ }^{21}-35.258(\mathrm{c}$ $\left.1.08, \mathrm{CHCl}_{3}\right)$.

\section{[1-(3-Bromo-2,2-dimethylpropoxy)-3,3-dimethylbutyl]benzene (4faa)}

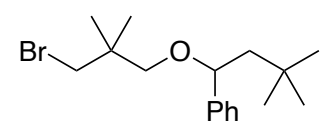

4faa

The reaction conditions were modified. The reaction was carried out with $\mathbf{1 f}(73.2 \mu \mathrm{l}, 0.6 \mathrm{mmol})$, 2a (22.9 $\mu \mathrm{l}, 0.2 \mathrm{mmol}), 3 \mathbf{3}(49.5 \mathrm{mg}, 0.2 \mathrm{mmol})$, PTH1 (0.79 mg, $0.002 \mathrm{mmol}, 1 \mathrm{~mol} \%)$, and LiBF 4 $(0.19 \mathrm{mg}, 0.002 \mathrm{mmol}, 1 \mathrm{~mol} \%)$ in $\mathrm{MeCN}(600 \mu \mathrm{L})$ under blue LED irradiation for $24 \mathrm{~h}$. The product 4faa was purified by flash chromatography on silica gel (100:0-95:5, hexane/EtOAc) (Scheme 2; $34.7 \mathrm{mg}, 0.11 \mathrm{mmol}, 53 \%$ isolated yield). Colorless oil. IR (neat) 700, 1062, 1100, 1247, 1364, 1474, 2868, $2954 \mathrm{~cm}^{-1} .{ }^{1} \mathbf{H}$ NMR $\left(600 \mathrm{MHz}, \mathrm{CDCl}_{3}\right) \delta 7.33-7.31(\mathrm{~m}, 2 \mathrm{H}), 7.26-7.23(\mathrm{~m}, 3 \mathrm{H}), 4.28(\mathrm{dd}, J$ $=9.0,2.4 \mathrm{~Hz}, 1 \mathrm{H}), 3.47-3.34(\mathrm{~m}, 2 \mathrm{H}), 3.06-2.97(\mathrm{~m}, 2 \mathrm{H}), 1.78(\mathrm{dd}, J=14.4,9.0 \mathrm{~Hz}, 1 \mathrm{H}), 1.38(\mathrm{dd}$, $J=14.4,2.4 \mathrm{~Hz}, 1 \mathrm{H}), 0.99$ (s, 12H), 0.98 (s, 3H). $\left.{ }^{13} \mathbf{C ~ N M R ~ ( 1 0 0 . 6 ~ M H z , ~} \mathrm{CDCl}_{3}\right) \delta 144.3,128.3$, $127.1,126.4,80.4,74.6,52.5,44.0,36.0,30.5,30.3,23.7,23.6$. HRMS-DART $(\mathrm{m} / \mathrm{z}):\left[\mathrm{M}+\mathrm{NH}_{4}\right]^{+}$ calcd for $\mathrm{C}_{17} \mathrm{H}_{31} \mathrm{BrNO}, 344.1584$; found, 344.1582 .

\section{1-[(3,3-Dimethyl-1-phenylbutoxy)methyl]-4-isopropylbenzene (4gaa)}

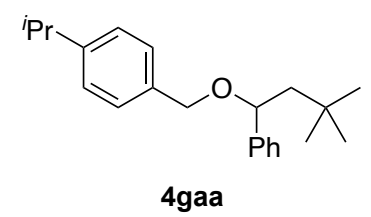

The product 4gaa was purified by flash chromatography on silica gel (100:0, hexane/EtOAc) (Scheme 2; $47.8 \mathrm{mg}, 0.15 \mathrm{mmol}, 77 \%$ isolated yield). Yellow oil. IR (neat) 700, 817, 1059, 1092, 1323, 1363, 1452, 2866, $2955 \mathrm{~cm}^{-1} .{ }^{1} \mathbf{H}$ NMR (400 MHz, $\left.\mathrm{CDCl}_{3}\right) \delta 7.37-7.29(\mathrm{~m}, 4 \mathrm{H}), 7.25-7.14(\mathrm{~m}$, $5 \mathrm{H}$ ), 4.44 (dd, $J=8.8,2.8 \mathrm{~Hz}, 1 \mathrm{H}), 4.26(\mathrm{~m}, 2 \mathrm{H}), 2.89$ (quin, $J=6.8 \mathrm{~Hz}, 1 \mathrm{H}), 1.88$ (dd, $J=14.4,8.8$ $\mathrm{Hz}, 1 \mathrm{H}), 1.47$ (dd, $J=14.4,2.8 \mathrm{~Hz}, 1 \mathrm{H}), 1.24(\mathrm{~s}, 3 \mathrm{H}), 1.23$ (s, 3H), 0.97 (s, 9H). ${ }^{13} \mathrm{C}$ NMR $(100.6$ $\left.\mathrm{MHz} \mathrm{CDCl}_{3}\right) \delta 148.1,144.2,135.9,128.4,128.0,172.2,126.6,126.3,79.7,70.4,52.2,33.9,30.6$, 30.2, 24.0. HRMS-DART (m/z): [M+NH$]^{+}$calcd for $\mathrm{C}_{22} \mathrm{H}_{34} \mathrm{NO}, 328.2635$; found, 328.2628.

\section{1-[(3,3-Dimethyl-1-phenylbutoxy)methyl]-4-iodobenzene (4haa)}

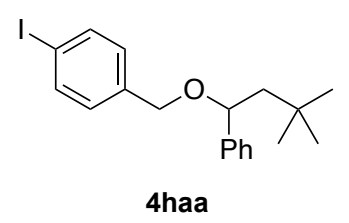

The reaction conditions were modified. The reaction was carried out with $\mathbf{1 h}$ ( $46.8 \mathrm{mg}, 0.2 \mathrm{mmol})$, 2a (45.8 $\mu \mathrm{l}, 0.4 \mathrm{mmol}), 3 \mathbf{a}$ (98.9 mg, $0.4 \mathrm{mmol}$ ), PTH1 (0.79 mg, $0.002 \mathrm{mmol}, 1 \mathrm{~mol} \%$ ), and $\mathrm{LiBF}_{4}$ $(0.19 \mathrm{mg}, 0.002 \mathrm{mmol}, 1 \mathrm{~mol} \%)$ in $\mathrm{MeCN}(600 \mu \mathrm{L})$ under blue LED irradiation for $24 \mathrm{~h}$. The product 
4haa was purified by flash chromatography on silica gel (100:0-90:10, hexane/EtOAc) (Scheme 2; $42.6 \mathrm{mg}, 0.11 \mathrm{mmol}, 54 \%$ isolated yield). Colorless oil. IR (neat) 700, 798, 1007, 1099, 1363, 1483, 2865, $2951 \mathrm{~cm}^{-1} .{ }^{1} \mathbf{H}$ NMR $\left(400 \mathrm{MHz}, \mathrm{CDCl}_{3}\right) \delta 7.64(\mathrm{~d}, J=7.2 \mathrm{~Hz}, 2 \mathrm{H}), 7.37-7.28(\mathrm{~m}, 5 \mathrm{H}), 7.04$ $(\mathrm{d}, J=7.6 \mathrm{~Hz}, 2 \mathrm{H}), 4.42(\mathrm{dd}, J=8.4,2.0 \mathrm{~Hz}, 1 \mathrm{H}), 4.28-4.17(\mathrm{~m}, 2 \mathrm{H}), 1.87(\mathrm{dd}, J=14.4,8.4 \mathrm{~Hz}, 1 \mathrm{H})$, $1.48(\mathrm{dd}, J=14.4,2.0 \mathrm{~Hz}, 1 \mathrm{H}), 0.96(\mathrm{~s}, 9 \mathrm{H}) .{ }^{13} \mathbf{C}$ NMR $\left(100.6 \mathrm{MHz}, \mathrm{CDCl}_{3}\right) \delta 143.7,138.3,137.3$, $129.7,128.5,127.4,126.6,92.8,79.9,69.7,52.1,30.6,30.2$. HRMS-DART $(\mathrm{m} / \mathrm{z}):\left[\mathrm{M}+\mathrm{NH}_{4}\right]^{+}$calcd for $\mathrm{C}_{19} \mathrm{H}_{27} \mathrm{INO}, 412.1132$; found, 412.1113 .

\section{[1-(Cyclohexyloxy)-3,3-dimethylbutyl]benzene (4iaa)}

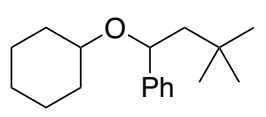

4iaa

The product 4iaa was purified by flash chromatography on silica gel (100:0, hexane/EtOAc) (Scheme 2; $28.6 \mathrm{mg}, 0.11 \mathrm{mmol}, 55 \%$ isolated yield). Pale yellow oil. IR (neat) 700, 1061, 1088, 1362, 1451, 2857, $2930 \mathrm{~cm}^{-1} .{ }^{1} \mathbf{H}$ NMR $\left(400 \mathrm{MHz}, \mathrm{CDCl}_{3}\right) \delta 7.33-7.21(\mathrm{~m}, 5 \mathrm{H}), 4.55(\mathrm{dd}, J=8.8$, $2.4 \mathrm{~Hz}, 1 \mathrm{H}), 3.09(\mathrm{~m}, 1 \mathrm{H}), 2.02(\mathrm{~m}, 1 \mathrm{H}), 1.79-1.63(\mathrm{~m}, 4 \mathrm{H}), 1.47-1.13(\mathrm{~m}, 7 \mathrm{H}), 0.97(\mathrm{~s}, 9 \mathrm{H}) .{ }^{13} \mathrm{C}$ NMR (100.6 MHz, $\left.\mathrm{CDCl}_{3}\right) \delta 145.7,128.2,126.9,126.4,76.6,74.9,52.7,33.8,31.6,30.7,30.2,25.8$, 24.4, 24.3. HRMS-DART ( $\mathrm{m} / \mathrm{z})$ : [M+NH$]^{+}$calcd for $\mathrm{C}_{18} \mathrm{H}_{32} \mathrm{NO}, 278.2478$; found, 278.2477.

\section{(3,3-Dimethyl-1-phenylbutoxy)cyclooctane (4jaa)}

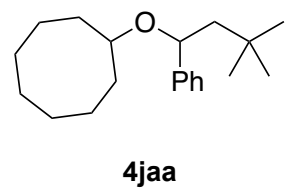

The reaction conditions were modified. The reaction was carried out with $\mathbf{1 j}(26.4 \mu \mathrm{l}, 0.2 \mathrm{mmol})$, 2a (45.8 $\mu 1,0.4 \mathrm{mmol}), 3 \mathbf{a}$ (98.9 mg, $0.4 \mathrm{mmol}$ ), PTH1 (0.79 mg, $0.002 \mathrm{mmol}, 1 \mathrm{~mol} \%$ ), and $\mathrm{LiBF}_{4}$ $(0.19 \mathrm{mg}, 0.002 \mathrm{mmol}, 1 \mathrm{~mol} \%)$ in $\mathrm{MeCN}(600 \mu \mathrm{L})$ under blue LED irradiation for $24 \mathrm{~h}$. The product 4jaa was purified by flash chromatography on silica gel (100:0-90:10, hexane/EtOAc) (Scheme 2; $47.3 \mathrm{mg}, 0.16 \mathrm{mmol}, 82 \%$ isolated yield). Colorless oil. IR (neat) 700, 736, 1057, 1087, 1363, 1473, 2863, $2919 \mathrm{~cm}^{-1} .{ }^{1} \mathbf{H}$ NMR $\left(400 \mathrm{MHz}, \mathrm{CDCl}_{3}\right) \delta 7.33-7.21(\mathrm{~m}, 5 \mathrm{H}), 4.48(\mathrm{dd}, J=8.4,2.8 \mathrm{~Hz}, 1 \mathrm{H})$, $3.25(\mathrm{~m}, 1 \mathrm{H}), 1.85-1.19(\mathrm{~m}, 16 \mathrm{H}), 0.96(\mathrm{~s}, 9 \mathrm{H}) .{ }^{13} \mathbf{C ~ N M R}\left(100.6 \mathrm{MHz}, \mathrm{CDCl}_{3}\right) \delta 145.5,128.2,126.9$, 126.6, 76.8, 76.3, 52.6, 32.5, 30.6, 30.3, 29.7, 27.6, 27.5, 25.3, 23.1, 22.8. HRMS-DART $(\mathrm{m} / z)$ : $\left[\mathrm{M}+\mathrm{NH}_{4}\right]^{+}$calcd for $\mathrm{C}_{20} \mathrm{H}_{36} \mathrm{NO}, 306.2791$; found, 306.2786 .

\section{4-(3,3-Dimethyl-1-phenylbutoxy)tetrahydro-2H-pyran (4kaa)}

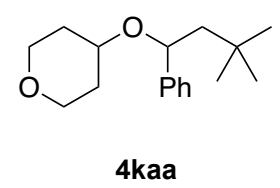

The reaction conditions were modified. The reaction was carried out with $\mathbf{1 k}(57.3 \mu \mathrm{l}, 0.6 \mathrm{mmol})$, 2a $(22.9 \mu \mathrm{l}, 0.2 \mathrm{mmol}), 3 \mathbf{3}$ (49.5 mg, $0.2 \mathrm{mmol})$, Molecular Sieves $3 \AA$ (50 mg), PTH1 (3.9 mg, 0.01 mmol, $5 \mathrm{~mol} \%$ ), and $\mathrm{LiBF}_{4}(0.9 \mathrm{mg}, 0.01 \mathrm{mmol}, 5 \mathrm{~mol} \%)$ in $\mathrm{MeCN}(600 \mu \mathrm{L})$ under blue LED 
irradiation for $12 \mathrm{~h}$. The product $4 \mathbf{k a a}$ was purified by flash chromatography on silica gel (100:0 80:20, hexane/EtOAc) (Scheme 2; $27.3 \mathrm{mg}, 0.10 \mathrm{mmol}, 52 \%$ isolated yield). Pale yellow oil. IR (neat) 701, 1002, 1090, 1363, 1453, 2863, $2950 \mathrm{~cm}^{-1} .{ }^{1} \mathbf{H} \mathbf{N M R}\left(400 \mathrm{MHz}, \mathrm{CDCl}_{3}\right) \delta 7.34-7.23(\mathrm{~m}$, $5 \mathrm{H}), 4.55(\mathrm{dd}, J=8.8,2.8 \mathrm{~Hz}, 1 \mathrm{H}), 3.97-3.81(\mathrm{~m}, 2 \mathrm{H}), 3.36-3.25(\mathrm{~m}, 3 \mathrm{H}), 1.96(\mathrm{~m}, 1 \mathrm{H}), 1.79(\mathrm{dd}, J$ $=14.8,8.8 \mathrm{~Hz}, 1 \mathrm{H}), 1.67-1.48(\mathrm{~m}, 3 \mathrm{H}), 1.40(\mathrm{dd}, J=2.7,14.5 \mathrm{~Hz}, 1 \mathrm{H}), 0.98(\mathrm{~s}, 9 \mathrm{H}) .{ }^{13} \mathbf{C} \mathbf{N M R}$ $\left(100.6 \mathrm{MHz} \mathrm{CDCl}_{3}\right) \delta 145.0,128.4,127.2,126.4,76.8,71.4,66.0,65.8,52.5,33.8,31.9,30.6,30.2$. HRMS-DART $(\mathrm{m} / \mathrm{z})$ : $\left[\mathrm{M}+\mathrm{NH}_{4}\right]^{+}$calcd for $\mathrm{C}_{17} \mathrm{H}_{30} \mathrm{NO}_{2}, 280.2271$; found, 280.2251 .

\section{4-(3,3-Dimethyl-1-phenylbutoxy)-1-tosylpiperidine (4laa)}

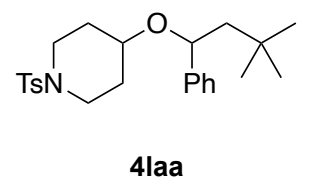

The product 4laa was purified by flash chromatography on silica gel (98:2-80:20, hexane/EtOAc) (Scheme 2; $49.9 \mathrm{mg}, 0.12 \mathrm{mmol}, 60 \%$ isolated yield). Colorless oil. IR (neat) 548, 572, 727, 932, 1039, 1089, 1164, 1347, $2949 \mathrm{~cm}^{-1} .{ }^{1} \mathbf{H}$ NMR (400 MHz, $\left.\mathrm{CDCl}_{3}\right) \delta 7.62-7.59(\mathrm{~m}, 2 \mathrm{H}), 7.30-7.18$ $(\mathrm{m}, 7 \mathrm{H}), 4.42(\mathrm{dd}, J=8.8,2.8 \mathrm{~Hz}, 1 \mathrm{H}), 3.31(\mathrm{~m}, 1 \mathrm{H}), 3.23-3.13(\mathrm{~m}, 2 \mathrm{H}), 2.75-2.62(\mathrm{~m}, 2 \mathrm{H}), 2.42(\mathrm{~s}$, $3 \mathrm{H}), 1.88(\mathrm{~m}, 1 \mathrm{H}), 1.75-1.57(\mathrm{~m}, 4 \mathrm{H}), 1.33(\mathrm{dd}, J=14.4,2.8 \mathrm{~Hz}, 1 \mathrm{H}), 0.87(\mathrm{~s}, 9 \mathrm{H}) .{ }^{13} \mathbf{C}$ NMR $(100.6$ $\left.\mathrm{MHz}, \mathrm{CDCl}_{3}\right) \delta 144.4,143.4,133.0,129.5,128.4,127.7,127.3,126.3,77.1,70.1,52.4,43.7,43.6$, 31.6, 30.5, 30.1, 29.2, 21.4. HRMS-DART $(\mathrm{m} / \mathrm{z})$ : $[\mathrm{M}+\mathrm{H}]^{+}$calcd for $\mathrm{C}_{24} \mathrm{H}_{34} \mathrm{NO}_{3} \mathrm{~S}, 416.2254$; found, 416.2254 .

\section{1-(3,3-Dimethyl-1-phenylbutoxy)adamantane (4maa)}

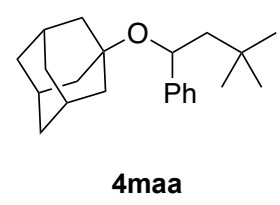

The reaction conditions were modified. The reaction was carried out with $1 \mathbf{m}(30.4 \mathrm{mg}, 0.2 \mathrm{mmol})$, 2a (45.8 $\mu 1,0.4 \mathrm{mmol}), 3 \mathbf{a}$ (98.9 mg, $0.4 \mathrm{mmol}$ ), PTH1 (0.79 mg, $0.002 \mathrm{mmol}, 1 \mathrm{~mol} \%$ ), and LiBF 4 $(0.19 \mathrm{mg}, 0.002 \mathrm{mmol}, 1 \mathrm{~mol} \%)$ in $\mathrm{MeCN}(600 \mu \mathrm{L})$ under blue LED irradiation for $24 \mathrm{~h}$. The product 4maa was purified by flash chromatography on silica gel (99:1-90:10, hexane/EtOAc) (Scheme 2; $38.1 \mathrm{mg}, 0.12 \mathrm{mmol}, 61 \%$ isolated yield). Yellow oil. IR (neat) 700, 1085, 1111, 1354, 1452, 2851, $2903 \mathrm{~cm}^{-1} .{ }^{1} \mathbf{H}$ NMR $\left(400 \mathrm{MHz}, \mathrm{CDCl}_{3}\right) \delta 7.33-7.27(\mathrm{~m}, 4 \mathrm{H}), 7.19(\mathrm{~m}, 1 \mathrm{H}), 4.76(\mathrm{dd}, J=8.4,3.6$ $\mathrm{Hz}, 1 \mathrm{H}), 2.02$ (br s, 3H), 1.77-1.52 (m, 13H), 1.37 (dd, $J=14.4,3.6 \mathrm{~Hz}, 1 \mathrm{H}), 0.95(\mathrm{~s}, 9 \mathrm{H}) .{ }^{13} \mathbf{C}$ NMR $\left(100.6 \mathrm{MHz}, \mathrm{CDCl}_{3}\right) \delta 148.6,128.0,126.4,126.3,73.8,71.0,53.9,43.3,36.4,30.7(\times 4 \mathrm{C}), 30.3$. HRMS-DART $(\mathrm{m} / \mathrm{z}):\left[\mathrm{M}+\mathrm{NH}_{4}\right]^{+}$calcd for $\mathrm{C}_{22} \mathrm{H}_{36} \mathrm{NO}, 330.2791$; found, 330.2785.

\section{\{3,3-Dimethyl-1-[(2-methyl-1-phenylpropan-2-yl)oxy]butyl\}benzene (4naa)}

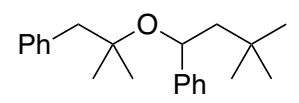


The reaction conditions were modified. The reaction was carried out with $1 \mathrm{n}(33.0 \mathrm{mg}, 0.2 \mathrm{mmol})$, 2a (45.8 $\mu 1,0.4 \mathrm{mmol}), 3 a(98.9 \mathrm{mg}, 0.4 \mathrm{mmol})$, PTH1 (0.79 mg, $0.002 \mathrm{mmol}, 1 \mathrm{~mol} \%$ ), and LiBF 4 $(0.19 \mathrm{mg}, 0.002 \mathrm{mmol}, 1 \mathrm{~mol} \%)$ in $\mathrm{MeCN}(600 \mu \mathrm{L})$ under blue LED irradiation for $24 \mathrm{~h}$. The product 4naa was purified by flash chromatography on silica gel (100:0-90:10, hexane/EtOAc) (Scheme 2; $33.5 \mathrm{mg}, 0.11 \mathrm{mmol}, 54 \%$ isolated yield). Yellow oil. IR (neat) 699, 759, 1027, 1144, 1221, 1364, 1453, $2951 \mathrm{~cm}^{-1} .{ }^{1} \mathbf{H}$ NMR $\left(400 \mathrm{MHz}, \mathrm{CDCl}_{3}\right) \delta 7.28-7.10(\mathrm{~m}, 10 \mathrm{H}), 4.67(\mathrm{dd}, J=8.4,4.4 \mathrm{~Hz}, 1 \mathrm{H})$, 2.77-2.67 (m, 2H), 1.77 (dd, $J=14.4,8.4 \mathrm{~Hz}, 1 \mathrm{H}), 1.45(\mathrm{dd}, J=14.4,4.4 \mathrm{~Hz}, 1 \mathrm{H}), 1.17$ (s, 3H), 0.92 $(\mathrm{s}, 9 \mathrm{H}), 0.83(\mathrm{~s}, 3 \mathrm{H}) .{ }^{13} \mathbf{C} \mathbf{N M R}\left(100.6 \mathrm{MHz}, \mathrm{CDCl}_{3}\right) \delta 148.1,138.6,130.8,128.0,127.6,126.5,126.5$, 125.9, 76.8, 72.7, 53.9, 49.8, 30.6, 30.3, 26.5, 25.9. HRMS-DART $(\mathrm{m} / \mathrm{z}):\left[\mathrm{M}+\mathrm{NH}_{4}\right]^{+}$calcd for $\mathrm{C}_{22} \mathrm{H}_{34} \mathrm{NO}, 328.2635$; found, 328.2635.

\section{$\{1-[(1-E t h y n y l c y c l o h e x y l) o x y]-3,3-d i m e t h y l b u t y l\}$ benzene (4oaa)}

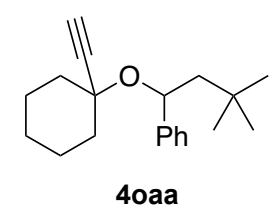

The reaction conditions were modified. The reaction was carried out with $\mathbf{1 o}(76.8 \mu 1,0.6 \mathrm{mmol})$, 2a (22.9 $\mu \mathrm{l}, 0.2 \mathrm{mmol}), 3 \mathbf{3}$ (49.5 mg, $0.2 \mathrm{mmol})$, PTH1 (3.9 mg, $0.01 \mathrm{mmol}, 5 \mathrm{~mol} \%)$, and $\mathrm{LiBF}_{4}(0.9$ $\mathrm{mg}, 0.01 \mathrm{mmol}, 5 \mathrm{~mol} \%)$ in $\mathrm{MeCN}(600 \mu \mathrm{L})$ under blue LED irradiation for $24 \mathrm{~h}$. The product $40 a a$ was purified by flash chromatography on silica gel (100:0, hexane/EtOAc) (Scheme 2; $38.1 \mathrm{mg}, 0.13$ mmol, 67\% isolated yield). Yellow oil. IR (neat) 628, 700, 1070, 1450, 2860, 2934, $3305 \mathrm{~cm}^{-1} .{ }^{1} \mathbf{H}$ NMR $\left(400 \mathrm{MHz}, \mathrm{CDCl}_{3}\right) \delta 7.34-7.25(\mathrm{~m}, 4 \mathrm{H}), 7.20(\mathrm{~m}, 1 \mathrm{H}), 5.05(\mathrm{dd}, J=8.4,3.6 \mathrm{~Hz}, 1 \mathrm{H}), 2.55(\mathrm{~s}$, $1 \mathrm{H}), 1.96(\mathrm{~m}, 1 \mathrm{H}), 1.84$ (dd, $J=14.4,8.4 \mathrm{~Hz}, 1 \mathrm{H}), 1.69-1.56(\mathrm{~m}, 3 \mathrm{H}), 1.47-1.27(\mathrm{~m}, 5 \mathrm{H}), 1.12-1.01$ $(\mathrm{m}, 2 \mathrm{H}), 0.96(\mathrm{~s}, 9 \mathrm{H}) .{ }^{13} \mathbf{C}$ NMR $\left(100.6 \mathrm{MHz} \mathrm{CDCl}_{3}\right) \delta 147.3,128.0,126.7,126.6,85.6,75.5,75.3$, 75.0, 53.3, 39.4, 38.9, 30.8, 30.3, 25.2, 23.2, 23.0. HRMS-DART $(\mathrm{m} / z)$ : $[\mathrm{M}+\mathrm{H}]^{+}$calcd for $\mathrm{C}_{20} \mathrm{H}_{29} \mathrm{O}$, 285.2213; found, 285.2207 .

\section{1-[1-(Cyclopentyloxy)-3,3-dimethylbutyl]-4-methoxybenzene (4aba)}

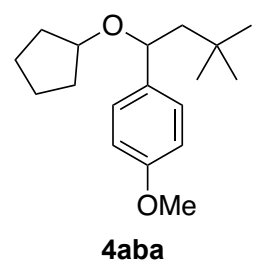

The product 4aba was purified by flash chromatography on silica gel (100:0-90:10, hexane/EtOAc) (Scheme 2; $40.9 \mathrm{mg}, 0.15 \mathrm{mmol}, 74 \%$ isolated yield). Pale red oil. IR (neat) 555, 830, $1037,1244,1510,1611,2869,2951 \mathrm{~cm}^{-1} .{ }^{1} \mathbf{H}$ NMR (400 MHz, $\left.\mathrm{CDCl}_{3}\right) \delta 7.22-7.19(\mathrm{~m}, 2 \mathrm{H}), 6.88-$ $6.84(\mathrm{~m}, 2 \mathrm{H}), 4.37(\mathrm{dd}, J=8.8,3.2 \mathrm{~Hz}, 1 \mathrm{H}), 3.80(\mathrm{~s}, 3 \mathrm{H}), 3.69$ (quin, $J=4.8 \mathrm{~Hz}, 1 \mathrm{H}), 1.77-1.36(\mathrm{~m}$, $10 \mathrm{H}), 0.95$ (s, 9H). ${ }^{13} \mathbf{C}$ NMR (100.6 MHz, $\left.\mathrm{CDCl}_{3}\right) \delta 158.6,137.2,127.7,113.6,78.2,77.1,55.2$, 52.5, 33.3, 31.1, 30.6, 30.2, 23.3, 23.3. HRMS-DART $(\mathrm{m} / \mathrm{z}):\left[\mathrm{M}+\mathrm{NH}_{4}\right]^{+}$calcd for $\mathrm{C}_{18} \mathrm{H}_{32} \mathrm{NO}_{2}$, 294.2428; found, 294.2416. 


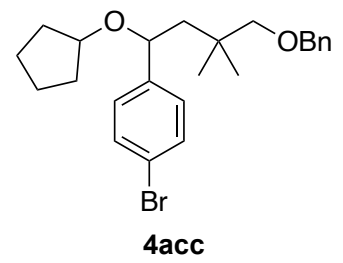

The product 4acc was purified by flash chromatography on silica gel (100:0-95:5, hexane/EtOAc) (Scheme 2; $40.6 \mathrm{mg}, 0.09 \mathrm{mmol}, 47 \%$ isolated yield). Pale yellow oil. IR (neat) 697, 735, 822, 1028, 1097, 1361, 1484, 2869, $2955 \mathrm{~cm}^{-1} .{ }^{1} \mathbf{H}$ NMR (400 MHz, $\left.\mathrm{CDCl}_{3}\right) \delta 7.42$ (d, J= 7.6 Hz, 2H), 7.36$7.27(\mathrm{~m}, 5 \mathrm{H}), 7.14(\mathrm{~d}, J=7.6 \mathrm{~Hz}, 2 \mathrm{H}), 4.53-4.45(\mathrm{~m}, 2 \mathrm{H}), 4.38(\mathrm{dd}, J=9.6,2.0 \mathrm{~Hz}, 1 \mathrm{H}), 3.63(\mathrm{~m}$, 1H), 3.31-3.15 (m, 2H), 1.75-1.38 (m, 10H), 0.99 (s, 6H). $\left.{ }^{13} \mathbf{C ~ N M R ~ ( 1 0 0 . 6 ~ M H z , ~ C D C l ~}\right)_{3} \delta 144.1$, 139.0, 131.3, 128.4, 128.2, 127.4, 127.3, 120.6, 79.5, 78.7, 76.8, 73.2, 47.9, 34.8, 33.3, 31.1, 25.6, 25.4, 23.3. HRMS-DART $(\mathrm{m} / z)$ : $[\mathrm{M}+\mathrm{H}]^{+}$calcd for $\mathrm{C}_{24} \mathrm{H}_{32} \mathrm{BrO}_{2}, 431.1580$; found, 431.1588 .

\section{1-[4-(Benzyloxy)-1-(cyclopentyloxy)-3,3-dimethylbutyl]-4-chlorobenzene (4adc)}

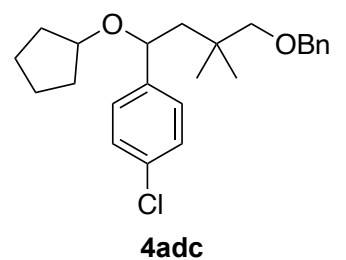

The product 4adc was purified by flash chromatography on silica gel (100:0-95:5, hexane/EtOAc) (Scheme 2; $38.7 \mathrm{mg}, 0.10 \mathrm{mmol}, 50 \%$ isolated yield). Pale yellow oil. IR (neat) 697, 734, 829, 1013, 1087, 1361, 1487, 2869, $2955 \mathrm{~cm}^{-1} .{ }^{1} \mathbf{H}$ NMR (400 MHz, $\left.\mathrm{CDCl}_{3}\right) \delta 7.36-7.25(\mathrm{~m}, 7 \mathrm{H}), 7.20-7.18(\mathrm{~m}$, 2H), 4.53-4.45 (m, 2H), 4.39 (dd, $J=9.2,2.8 \mathrm{~Hz}, 1 \mathrm{H}), 3.63(\mathrm{~m}, 1 \mathrm{H}), 3.25-3.15(\mathrm{~m}, 2 \mathrm{H}), 1.76-1.37$ $(\mathrm{m}, 10 \mathrm{H}), 0.99$ (s, 3H), 0.99 (s, 3H). ${ }^{13} \mathbf{C}$ NMR $\left(100.6 \mathrm{MHz}, \mathrm{CDCl}_{3}\right) \delta 143.6,139.0,132.5,128.4$, $128.2,128.0,127.4,127.3,79.4,78.7,76.7,73.2,47.9,34.8,33.3,31.1,25.6,25.4,23.3$. HRMSDART $(\mathrm{m} / \mathrm{z}):[\mathrm{M}+\mathrm{H}]^{+}$calcd for $\mathrm{C}_{24} \mathrm{H}_{32} \mathrm{ClO}_{2}, 387.2085$; found, 387.2092 .

\section{1-[4-(Benzyloxy)-1-(cyclopentyloxy)-3,3-dimethylbutyl]-3,5-dimethylbenzene (4aec)}

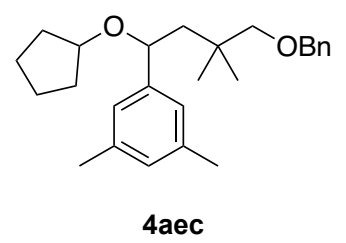

The product 4aec was purified by flash chromatography on silica gel (100:0, hexane/EtOAc) (Scheme 2; $42.6 \mathrm{mg}, 0.11 \mathrm{mmol}, 56 \%$ isolated yield). Pale yellow oil. IR (neat) 697, 734, 849, 1092, 1360, 1453, 1608, 2868, $2953 \mathrm{~cm}^{-1} .{ }^{1} \mathbf{H}$ NMR (400 MHz, $\left.\mathrm{CDCl}_{3}\right) \delta 7.36-7.24(\mathrm{~m}, 5 \mathrm{H}), 6.87(\mathrm{~s}, 2 \mathrm{H})$, $6.86(\mathrm{~s}, 1 \mathrm{H}), 4.54-4.46(\mathrm{~m}, 2 \mathrm{H}), 4.36(\mathrm{dd}, J=9.6,2.4 \mathrm{~Hz}, 1 \mathrm{H}), 3.68$ (quin, $J=4.8 \mathrm{~Hz}, 1 \mathrm{H}), 3.29$ $3.17(\mathrm{~m}, 2 \mathrm{H}), 2.29$ (s, 6H), $1.75(\mathrm{dd}, J=14.4,9.6 \mathrm{~Hz}, 1 \mathrm{H}), 1.69-1.38(\mathrm{~m}, 9 \mathrm{H}), 1.01(\mathrm{~s}, 3 \mathrm{H}), 1.08$ (s, $3 \mathrm{H}) .{ }^{13} \mathrm{C}$ NMR $\left(100.6 \mathrm{MHz}, \mathrm{CDCl}_{3}\right) \delta 144.9,139.1,137.6,128.6,128.2,127.4,127.2,124.4,79.6$, 78.4, 77.2, 73.2, 48.1, 34.9, 33.3, 31.0, 25.6, 25.4, 23.3, 23.3, 21.3. HRMS-DART $(\mathrm{m} / \mathrm{z}):[\mathrm{M}+\mathrm{H}]^{+}$ calcd for $\mathrm{C}_{26} \mathrm{H}_{37} \mathrm{O}_{2}, 381.2788$; found, 381.2789. 


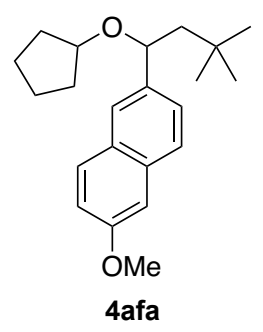

The product 4afa was purified by flash chromatography on silica gel (100:0-90:10, hexane/EtOAc) (Scheme 2; $43.1 \mathrm{mg}, 0.13 \mathrm{mmol}, 66 \%$ isolated yield). Pale yellow oil. IR (neat) 475, 851, 1033, 1167, 1264, 1482, 1607, $2950 \mathrm{~cm}^{-1} .{ }^{1} \mathbf{H}$ NMR $\left(400 \mathrm{MHz} \mathrm{CDCl}_{3}\right) \delta 7.71(\mathrm{~d}, J=8.4 \mathrm{~Hz}, 2 \mathrm{H}), 7.63(\mathrm{~s}, 1 \mathrm{H})$, $7.43(\mathrm{dd}, J=8.4,1.6 \mathrm{~Hz}, 1 \mathrm{H}), 7.15-7.13(\mathrm{~m}, 2 \mathrm{H}), 4.55$ (dd, $J=8.8,3.2 \mathrm{~Hz}, 1 \mathrm{H}), 3.92$ (s, 3H), 3.74 (quin, $J=4.8 \mathrm{~Hz}, 1 \mathrm{H}), 1.87-1.37(\mathrm{~m}, 10 \mathrm{H}), 0.99(\mathrm{~s}, 9 \mathrm{H}) .{ }^{13} \mathbf{C} \mathbf{N M R}\left(100.6 \mathrm{MHz}, \mathrm{CDCl}_{3}\right) \delta 157.4$, 140.3, 133.9, 129.2, 128.7, 127.0, 125.5, 125.1, 118.7, 105.7, 78.5, 77.8, 55.3, 52.3, 33.3, 31.2, 30.7, 30.3, 23.3, 23.3. HRMS-DART $(\mathrm{m} / \mathrm{z}):[\mathrm{M}+\mathrm{H}]^{+}$calcd for $\mathrm{C}_{22} \mathrm{H}_{31} \mathrm{O}_{2}, 327.2319$; found, 327.2319.

\section{2-[4-(Benzyloxy)-1-(cyclopentyloxy)-3,3-dimethylbutyl]thiophene (4agc)}

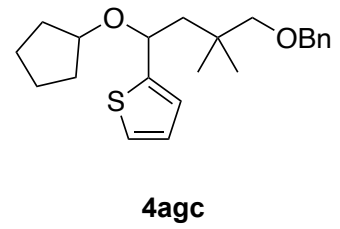

The reaction conditions were modified. The reaction was carried out with $\mathbf{1 a}(54.5 \mu \mathrm{l}, 0.6 \mathrm{mmol})$, $\mathbf{2 g}$ (21.2 $\mu \mathrm{l}, 0.2 \mathrm{mmol}), 3 \mathbf{c}$ (70.7 mg, $0.2 \mathrm{mmol}$ ), PTH1 (0.79 mg, $0.002 \mathrm{mmol}, 1 \mathrm{~mol} \%$ ), and LiBF 4 $(0.19 \mathrm{mg}, 0.002 \mathrm{mmol}, 1 \mathrm{~mol} \%)$ in MeCN $(600 \mu \mathrm{L})$ under blue LED irradiation for $4 \mathrm{~h}$. The product 4agc was purified by flash chromatography on silica gel (100:0-95:5, hexane/EtOAc) (Scheme 2; $48.8 \mathrm{mg}, 0.14 \mathrm{mmol}, 68 \%$ isolated yield). Pale yellow oil. IR (neat) 696, 735, 1092, 1362, 1453, 2868, $2954 \mathrm{~cm}^{-1} .{ }^{1} \mathbf{H}$ NMR $\left(400 \mathrm{MHz}, \mathrm{CDCl}_{3}\right) \delta 7.34-7.21(\mathrm{~m}, 6 \mathrm{H}), 6.93-6.88(\mathrm{~m}, 2 \mathrm{H}), 4.70(\mathrm{dd}, J=8.8$, $3.2 \mathrm{~Hz}, 1 \mathrm{H}), 4.54-4.45$ (m, 2H), 3.79 (quin, $J=4.4 \mathrm{~Hz}, 1 \mathrm{H}), 3.25-3.16(\mathrm{~m}, 2 \mathrm{H}), 1.91$ (dd, $J=14.8$, $8.8 \mathrm{~Hz}, 1 \mathrm{H}), 1.75-1.41(\mathrm{~m}, 9 \mathrm{H}), 0.99(\mathrm{~s}, 6 \mathrm{H}) .{ }^{13} \mathbf{C ~ N M R}\left(100.6 \mathrm{MHz}, \mathrm{CDCl}_{3}\right) \delta 149.3,139.0,128.2$, 127.4, 127.3, 126.0, 124.2, 124.1, 79.4, 78.5, 73.2, 72.8, 48.4, 34.8, 33.2, 31.1, 25.4, 25.3, 23.4. HRMS-DART $(\mathrm{m} / \mathrm{z})$ : $\left[\mathrm{M}+\mathrm{NH}_{4}\right]^{+}$calcd for $\mathrm{C}_{22} \mathrm{H}_{34} \mathrm{NO}_{2} \mathrm{~S}, 376.2305$; found, 376.2304 .

\section{[5-(Benzyloxy)-2-(cyclopentyloxy)-4,4-dimethylpentan-2-yl]benzene (4ahc)}

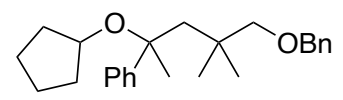

4ahc

The reaction conditions were modified. The reaction was carried out with $\mathbf{1 a}(54.5 \mu 1,0.6 \mathrm{mmol})$, 2h (26.0 mg, $0.2 \mathrm{mmol}), 3 \mathbf{c}$ (70.7 mg, $0.2 \mathrm{mmol}$ ), PTH1 (0.79 mg, $0.002 \mathrm{mmol}, 1 \mathrm{~mol} \%$ ), and LiBF $(0.19 \mathrm{mg}, 0.002 \mathrm{mmol}, 1 \mathrm{~mol} \%)$ in MeCN $(600 \mu \mathrm{L})$ under blue LED irradiation for $4 \mathrm{~h}$. The product 4ahc was purified by flash chromatography on silica gel (100:0-90:10, hexane/EtOAc) (Scheme 2; $44.7 \mathrm{mg}, 0.12 \mathrm{mmol}, 61 \%$ isolated yield). Colorless oil. IR (neat) 698, 734, 1028, 1070, 1097, 1361, 
1445, 2867, $2954 \mathrm{~cm}^{-1} .{ }^{1} \mathbf{H}$ NMR (400 MHz, $\left.\mathrm{CDCl}_{3}\right) \delta 7.41-7.19(\mathrm{~m}, 10 \mathrm{H}), 4.37(\mathrm{~s}, 2 \mathrm{H}), 3.67$ (quin, $J=5.6 \mathrm{~Hz}, 1 \mathrm{H}), 3.05-2.97(\mathrm{~m}, 2 \mathrm{H}), 1.86-1.26(\mathrm{~m}, 13 \mathrm{H}), 0.88(\mathrm{~s}, 3 \mathrm{H}), 0.84(\mathrm{~s}, 3 \mathrm{H}) .{ }^{13} \mathrm{C}$ NMR $(100.6$ $\left.\mathrm{MHz}_{2} \mathrm{CDCl}_{3}\right) \delta 148.1,139.2,128.1,127.7,127.2,127.1,126.7,126.3,80.2,80.0,74.8,72.9,53.1$, $35.8,35.1,34.4,26.6,26.5,25.7,24.0,23.4$. HRMS-DART $(\mathrm{m} / \mathrm{z}):[\mathrm{M}+\mathrm{H}]^{+}$calcd for $\mathrm{C}_{25} \mathrm{H}_{35} \mathrm{O}_{2}$, 367.2632; found, 367.2633 .

[1-(2-Ethoxyethoxy)-3,3-dimethylbutane-1,1-diyl]dibenzene (4dia)

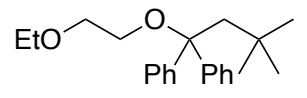

4dia

The product 4dia was purified by flash chromatography on silica gel (100:0-90:10, hexane/Et $2 \mathrm{O})$ (Scheme 2; $43.1 \mathrm{mg}, 0.13 \mathrm{mmol}, 66 \%$ isolated yield). Colorless oil. IR (neat) 699, 747, 1085, 1118, 1364, 1446, 2867, $2950 \mathrm{~cm}^{-1} .{ }^{1} \mathbf{H}$ NMR (400 MHz, $\left.\mathrm{CDCl}_{3}\right) \delta 7.37-7.35(\mathrm{~m}, 4 \mathrm{H}), 7.26-7.22(\mathrm{~m}, 4 \mathrm{H})$, $7.18-7.13(\mathrm{~m}, 2 \mathrm{H}), 3.58(\mathrm{t}, J=6.0 \mathrm{~Hz}, 2 \mathrm{H}), 3.51(\mathrm{q}, J=6.8 \mathrm{~Hz}, 2 \mathrm{H}), 3.27(\mathrm{t}, J=6.0 \mathrm{~Hz}, 2 \mathrm{H}), 2.38$ $(\mathrm{s}, 2 \mathrm{H}), 1.18(\mathrm{t}, J=6.8 \mathrm{~Hz}, 3 \mathrm{H}), 0.75(\mathrm{~s}, 9 \mathrm{H}) .{ }^{13} \mathbf{C ~ N M R}\left(100.6 \mathrm{MHz}, \mathrm{CDCl}_{3}\right) \delta 146.7,127.6,127.3$, $126.3,82.4,69.7,68.0,66.5,61.5,45.5,31.5,31.1,25.6,15.2$. HRMS-DART $(\mathrm{m} / \mathrm{z}):\left[\mathrm{M}+\mathrm{NH}_{4}\right]^{+}$ calcd for $\mathrm{C}_{22} \mathrm{H}_{34} \mathrm{NO}_{2}, 344.2584$; found, 344.2592 .

\section{Methyl 2-[(Cyclopentyloxy)(phenyl)methyl]-3,3-dimethylbutanoate (anti) (4aja)}

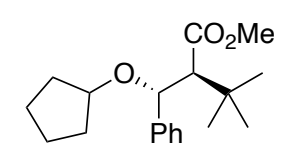

4aja

The product 4aja was purified by flash chromatography on silica gel (100:0-90:10, hexane/EtOAc) (Scheme 2; $35.9 \mathrm{mg}, 0.12 \mathrm{mmol}, 59 \%$ isolated yield). An alternative synthesis of 4aja confirmed the anti configuration of 4aja. Pale yellow oil. IR (neat) 702, 1048, 1149, 1260, 1326, 1366, 1433, 1731, 2871, $2954 \mathrm{~cm}^{-1} .{ }^{1} \mathbf{H}$ NMR (400 MHz, $\left.\mathrm{CDCl}_{3}\right) \delta 7.39-7.27(\mathrm{~m}, 5 \mathrm{H}), 4.54(\mathrm{~d}, J=10.4 \mathrm{~Hz}, 1 \mathrm{H}), 3.69$ (s, 3H), 3.63 (quin, $J=4.4 \mathrm{~Hz}, 1 \mathrm{H}), 2.74(\mathrm{~d}, J=10.4 \mathrm{~Hz}, 1 \mathrm{H}), 1.74-1.34(\mathrm{~m}, 8 \mathrm{H}), 0.73(\mathrm{~s}, 9 \mathrm{H}) .{ }^{13} \mathrm{C}$ NMR (100.6 MHz, $\left.\mathrm{CDCl}_{3}\right) \delta 174.3,141.3,128.6,128.2,128.1,79.2,78.1,62.6,50.9,33.3,31.9$, 30.5, 28.6, 23.3, 23.2. HRMS-DART $(\mathrm{m} / \mathrm{z})$ : $[\mathrm{M}+\mathrm{H}]^{+}$calcd for $\mathrm{C}_{19} \mathrm{H}_{29} \mathrm{O}_{3}, 305.2111$; found, 305.2111 .

\section{3-(tert-Butyl)-4-(cyclopentyloxy)chroman-2-one (anti) (4aka)}

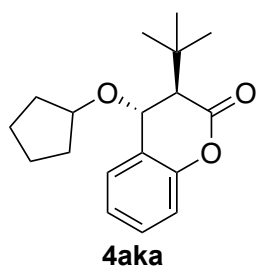

The product 4aka was purified by flash chromatography on silica gel (100:0-90:10, hexane/EtOAc) (Scheme 2; $27.1 \mathrm{mg}, 0.09 \mathrm{mmol}, 47 \%$ isolated yield). The anti configuration of 4aka was assigned by consideration of the stereochemical pathway. Pale yellow oil. IR (neat) 758, 1040, 1148, 1222, 1459, 1765, $2959 \mathrm{~cm}^{-1} .{ }^{1} \mathbf{H}$ NMR (400 MHz, $\left.\mathrm{CDCl}_{3}\right) \delta 7.35$ (dt, $\left.J=7.2,1.2 \mathrm{~Hz}, 1 \mathrm{H}\right)$, 
$7.24(\mathrm{dd}, J=7.2,1.2 \mathrm{~Hz}, 1 \mathrm{H}), 7.13(\mathrm{dt}, J=7.2,1.2 \mathrm{~Hz}, 1 \mathrm{H}), 7.05(\mathrm{~d}, J=8.0 \mathrm{~Hz}, 1 \mathrm{H}), 4.51(\mathrm{~d}, J=2.0$ $\mathrm{Hz}, 1 \mathrm{H}), 3.92(\mathrm{~m}, 1 \mathrm{H}), 2.88(\mathrm{~d}, J=2.0 \mathrm{~Hz}, 1 \mathrm{H}), 1.77-1.38(\mathrm{~m}, 8 \mathrm{H}), 0.88(\mathrm{~s}, 9 \mathrm{H}) .{ }^{13} \mathrm{C}$ NMR $(100.6$ $\left.\mathrm{MHz}, \mathrm{CDCl}_{3}\right) \delta 168.3,152.2,130.2,128.9,124.0,122.2,116.8,77.8,71.8,56.6,33.0,32.8,31.9$, 28.8, 23.6, 23.5. HRMS-DART $(\mathrm{m} / \mathrm{z})$ : $[\mathrm{M}+\mathrm{H}]^{+}$calcd for $\mathrm{C}_{18} \mathrm{H}_{25} \mathrm{O}_{3}, 289.1798$; found, 289.1797 .

\section{2-[(Cyclopentyloxy)(phenyl)methyl]-3,3-dimethylbutanal (anti) (4ala)}

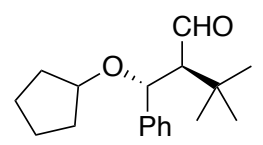

4ala

The reaction conditions were modified. The reaction was carried out with $\mathbf{1 a}(54.5 \mu 1,0.6 \mathrm{mmol})$, 21 (25.2 $\mu 1,0.2 \mathrm{mmol}), 3 \mathbf{a}$ (49.5 mg, $0.2 \mathrm{mmol})$, PTH1 (0.79 mg, $0.002 \mathrm{mmol}, 1 \mathrm{~mol} \%$ ), and LiBF 4 $(0.19 \mathrm{mg}, 0.002 \mathrm{mmol}, 1 \mathrm{~mol} \%)$ in $\mathrm{MeCN}(600 \mu \mathrm{L})$ under blue LED irradiation for $12 \mathrm{~h}$. Reaction was carried out for $12 \mathrm{~h}$. The product 4ala was purified by flash chromatography on silica gel (100:080:20, hexane/EtOAc) (Scheme 2; $17.0 \mathrm{mg}, 0.06 \mathrm{mmol}, 31 \%$ isolated yield). The anti configuration of 4ala was assigned by consideration of the stereochemical pathway. Colorless oil. IR (neat) 702, 769, 1050, 1367, 1703, 2871, $2958 \mathrm{~cm}^{-1} .{ }^{1} \mathbf{H}$ NMR (400 MHz, $\left.\mathrm{CDCl}_{3}\right) \delta 9.86(\mathrm{~d}, J=5.2 \mathrm{~Hz}, 1 \mathrm{H})$, 7.35-7.25 (m, 5H), $4.82(\mathrm{~d}, J=5.2 \mathrm{~Hz}, 1 \mathrm{H}), 3.75$ (quin, $J=4.4 \mathrm{~Hz}, 1 \mathrm{H}), 2.20(\mathrm{t}, J=5.2 \mathrm{~Hz}, 1 \mathrm{H}$ ), $1.77-1.41(\mathrm{~m}, 8 \mathrm{H}), 0.98(\mathrm{~s}, 9 \mathrm{H}) .{ }^{13} \mathbf{C} \mathbf{N M R}\left(100.6 \mathrm{MHz}, \mathrm{CDCl}_{3}\right) \delta 204.9,141.8,128.5,127.8,127.4$, 78.7, 78.2, 67.3, 33.2, 32.9, 30.9, 28.8, $23.3(\times 2 \mathrm{C})$. HRMS-DART $(\mathrm{m} / \mathrm{z}):[\mathrm{M}+\mathrm{H}]^{+}$calcd for $\mathrm{C}_{18} \mathrm{H}_{27} \mathrm{O}_{2}$, 275.2006; found, 275.2008 .

\section{2-[1-(Cyclopentyloxy)-2,2-difluoro-3,3-dimethylbutyl]naphthalene (4ama)}

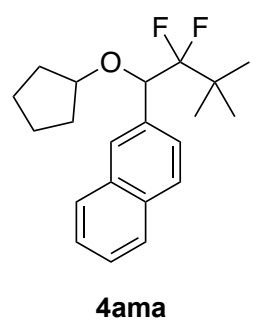

The reaction conditions were modified. The reaction was carried out with $\mathbf{1 a}(54.5 \mu \mathrm{l}, 0.6 \mathrm{mmol})$, 2m (38.0 mg, $0.2 \mathrm{mmol}), 3 \mathrm{a}$ (49.5 mg, $0.2 \mathrm{mmol}$ ), PTH1 (0.79 mg, $0.002 \mathrm{mmol}, 1 \mathrm{~mol} \%$ ), and LiBF 4 $(0.19 \mathrm{mg}, 0.002 \mathrm{mmol}, 1 \mathrm{~mol} \%)$ in $\mathrm{MeCN}(600 \mu \mathrm{L})$ under blue LED irradiation for $12 \mathrm{~h}$. The product 4ama was purified by flash chromatography on silica gel (100:0-80:20, hexane/EtOAc) (Scheme 2; $15.3 \mathrm{mg}, 0.06 \mathrm{mmol}, 23 \%$ isolated yield). White solid. M.p. $102-104{ }^{\circ} \mathrm{C}$. IR (neat) 479, 759, 803, 1009, 1060, 1089, 1162, 1367, 1484, $2964 \mathrm{~cm}^{-1} .{ }^{1} \mathbf{H}$ NMR (400 MHz, $\left.\mathrm{CDCl}_{3}\right) \delta 7.86-7.83(\mathrm{~m}, 4 \mathrm{H})$, $7.58(\mathrm{~d}, J=8.4 \mathrm{~Hz}, 1 \mathrm{H}), 7.50-7.46(\mathrm{~m}, 2 \mathrm{H}), 4.81(\mathrm{dd}, J=20.8,3.6 \mathrm{~Hz}, 1 \mathrm{H}), 3.84(\mathrm{~m}, 1 \mathrm{H}), 1.82-1.42$ $(\mathrm{m}, 8 \mathrm{H}), 1.17(\mathrm{~s}, 9 \mathrm{H}) .{ }^{13} \mathbf{C}$ NMR $\left(100.6 \mathrm{MHz}, \mathrm{CDCl}_{3}\right) \delta 134.0,133.4,132.9,128.7,128.0,127.7$, $127.5,126.7,126.0,125.9,79.7,79.5\left(\mathrm{dd}, J_{\mathrm{C}-\mathrm{F}}=37.2,24.0 \mathrm{~Hz}\right), 38.6\left(\mathrm{t}, J_{\mathrm{C}-\mathrm{F}}=23.2 \mathrm{~Hz}\right), 33.1,30.8$, $24.9\left(\mathrm{t}, J_{\mathrm{C}-\mathrm{F}}=4.4 \mathrm{~Hz}\right), 23.3$. HRMS-DART $(\mathrm{m} / \mathrm{z}):\left[\mathrm{M}+\mathrm{NH}_{4}\right]^{+}$calcd for $\mathrm{C}_{21} \mathrm{H}_{30} \mathrm{~F}_{2} \mathrm{NO}, 350.2290$; found, 350.2302 . 


\section{- Procedure for Three Component Coupling with Other Heteroatom Nucleophiles}

The reaction with other heteroatom nucleophiles (Scheme 3). In a glovebox, to an oven-dried vial with a stirring bar was added PTH1 $(3.9 \mathrm{mg}, 0.01 \mathrm{mmol}$ or $0.79 \mathrm{mg}, 0.002 \mathrm{mmol})$, LiBF (0.9 mg, $0.01 \mathrm{mmol}$ or $0.19 \mathrm{mg}, 0.002 \mathrm{mmol})$, redox active ester $3(0.2$ or $0.3 \mathrm{mmol})$, styrene $2 \mathrm{a}(0.2 \mathrm{mmol})$ and heteroatom nucleophile 5-9 $(0.6 \mathrm{mmol})$ and solvent $(600 \mu \mathrm{l})$. After sealing the vial with a cap and removed from the glove box, the reaction was stirred and irradiated with a $34 \mathrm{~W}$ blue LED (0.5 cm away) with a cooling fan to keep the temperature around $40{ }^{\circ} \mathrm{C}$. After $12-14 \mathrm{~h}$, the reaction was quenched with a short plug of silica gel using diethyl ether. After volatiles were removed under reduced pressure, purification by flash column chromatography on silica gel or aluminum oxide gave the coupling product.

\section{- Characterization Data for Three Component Coupling Products}

\section{3,3-Dimethyl-1-phenylbutan-1-ol (10aaa)}

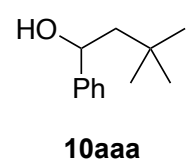

The reaction was carried out with water $\mathbf{5 a}(60 \mu \mathrm{l}), \mathbf{2 a}(22.9 \mu \mathrm{l}, 0.2 \mathrm{mmol}), \mathbf{3 a}(49.5 \mathrm{mg}, 0.2 \mathrm{mmol})$, PTH1 (0.79 mg, $0.002 \mathrm{mmol}, 1 \mathrm{~mol} \%$ ), and $\mathrm{LiBF}_{4}$ (0.19 mg, $0.002 \mathrm{mmol}, 1 \mathrm{~mol} \%$ ) in MeCN (540 $\mu \mathrm{L})$ under blue LED irradiation for $12 \mathrm{~h}$. The product 10aaa was purified by flash chromatography on silica gel (100:0-90:10, hexane/EtOAc) (Scheme 3; $25.7 \mathrm{mg}, 0.14 \mathrm{mmol}, 72 \%$ isolated yield). Pale yellow oil. The spectrum data of product 10aaa was consistent with the literature. ${ }^{14}$

\section{3,3-Dimethyl-1-phenylbutyl Benzoate (11aaa)}

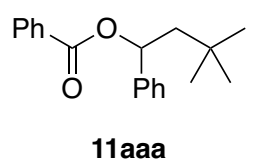

The reaction was carried out with $\mathbf{6 a}(73.3 \mathrm{mg}, 0.6 \mathrm{mmol}), \mathbf{2 a}(22.9 \mu 1,0.2 \mathrm{mmol}), \mathbf{3 a}(49.5 \mathrm{mg}$, $0.2 \mathrm{mmol}$ ), PTH1 (3.9 mg, $0.01 \mathrm{mmol}, 5 \mathrm{~mol} \%$ ), and $\mathrm{LiBF}_{4}(0.9 \mathrm{mg}, 0.01 \mathrm{mmol}, 5 \mathrm{~mol} \%$ ) in THF $(600 \mu \mathrm{L})$ under blue LED irradiation for $12 \mathrm{~h}$. The product 11aaa was purified by flash chromatography on silica gel (100:0-95:5, hexane/EtOAc) (Scheme 3; $29.3 \mathrm{mg}, 0.10 \mathrm{mmol}, 52 \%$ isolated yield). Yellow oil. IR (neat) 546, 698, 710, 1109, 1271, 1716, $2954 \mathrm{~cm}^{-1} .{ }^{1} \mathbf{H}$ NMR (400 $\left.\mathrm{MHz} \mathrm{CDCl}_{3}\right) \delta 8.07(\mathrm{~d}, J=8.0 \mathrm{~Hz}, 2 \mathrm{H}), 7.55(\mathrm{~m}, 1 \mathrm{H}), 7.46-7.39(\mathrm{~m}, 4 \mathrm{H}), 7.32(\mathrm{t}, J=8.0 \mathrm{~Hz}, 2 \mathrm{H})$, $7.24(\mathrm{~m}, 1 \mathrm{H}), 6.12(\mathrm{dd}, J=9.2,2.8 \mathrm{~Hz}, 1 \mathrm{H}), 2.14(\mathrm{dd}, J=14.8,9.2 \mathrm{~Hz}, 1 \mathrm{H}), 1.72(\mathrm{dd}, J=14.8,2.8$ $\mathrm{Hz}, 1 \mathrm{H}), 1.00$ (s, 9H). ${ }^{13} \mathrm{C}$ NMR $\left(100.6 \mathrm{MHz}, \mathrm{CDCl}_{3}\right) \delta 165.7,142.5,132.9,130.5,129.6,128.5$, $128.3,127.7,126.2,74.6,50.3,30.5,30.0$. HRMS-DART $(\mathrm{m} / \mathrm{z}):\left[\mathrm{M}+\mathrm{NH}_{4}\right]^{+}$calcd for $\mathrm{C}_{19} \mathrm{H}_{26} \mathrm{NO}_{2}$, 300.1958; found, 300.1958 .

\section{3,3-Dimethyl-1-phenylbutyl 2-(Thiophen-2-yl)acetate (11baa)}

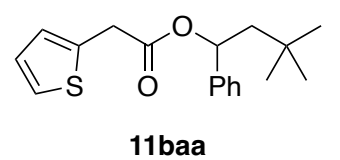


The reaction was carried out with $\mathbf{6 b}(85.3 \mathrm{mg}, 0.6 \mathrm{mmol}), \mathbf{2 a}(22.9 \mu 1,0.2 \mathrm{mmol}), \mathbf{3 a}(49.5 \mathrm{mg}$, $0.2 \mathrm{mmol}$ ), PTH1 (3.9 mg, $0.01 \mathrm{mmol}, 5 \mathrm{~mol} \%$ ), and LiBF 4 (0.9 mg, $0.01 \mathrm{mmol}, 5 \mathrm{~mol} \%$ ) in THF $(600 \mu \mathrm{L})$ under blue LED irradiation for $12 \mathrm{~h}$. The product 11 baa was purified by flash chromatography on silica gel (100:0-95:5, hexane/EtOAc) (Scheme 3; $21.2 \mathrm{mg}, 0.07 \mathrm{mmol}, 35 \%$ isolated yield). Pale yellow oil. IR (neat) 697, 966, 1166, 1236, 1366, 1735, $2954 \mathrm{~cm}^{-1}$. ${ }^{1} \mathbf{H}$ NMR $\left(400 \mathrm{MHz}, \mathrm{CDCl}_{3}\right) \delta 7.33-7.23(\mathrm{~m}, 5 \mathrm{H}), 7.20(\mathrm{dd}, J=5.2,1.2 \mathrm{~Hz}, 1 \mathrm{H}), 6.94-6.89(\mathrm{~m}, 2 \mathrm{H}), 5.89$ (dd, $J=8.8,3.6 \mathrm{~Hz}, 1 \mathrm{H}), 3.81(\mathrm{~s}, 2 \mathrm{H}), 1.96(\mathrm{dd}, J=14.8,8.8 \mathrm{~Hz}, 1 \mathrm{H}), 1.62(\mathrm{dd}, J=14.8,3.6 \mathrm{~Hz}, 1 \mathrm{H})$, 0.91 (s, 9H). ${ }^{13} \mathbf{C}$ NMR (100.6 MHz, $\left.\mathrm{CDCl}_{3}\right) \delta 169.5,142.0,134.9,128.4,127.7,126.8,126.7,126.3$, 124.9, 75.0, 49.8, 35.8, 30.4, 29.8. HRMS-DART $(\mathrm{m} / \mathrm{z})$ : $[\mathrm{M}+\mathrm{H}]^{+}$calcd for $\mathrm{C}_{18} \mathrm{H}_{23} \mathrm{O}_{2} \mathrm{~S}, 303.1413$; found, 303.1406 .

\section{3,3-Dimethyl-1-phenylbutyl 2-(4-Chlorophenyl)acetate (11 caa)}

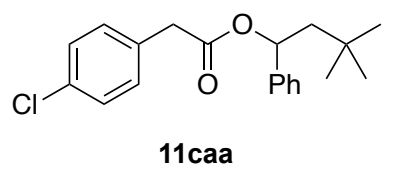

The reaction was carried out with $\mathbf{6 c}(102.4 \mathrm{mg}, 0.6 \mathrm{mmol}), \mathbf{2 a}(22.9 \mu \mathrm{l}, 0.2 \mathrm{mmol}), \mathbf{3 a}(49.5 \mathrm{mg}$, $0.2 \mathrm{mmol}$ ), PTH1 (3.9 mg, $0.01 \mathrm{mmol}, 5 \mathrm{~mol} \%$ ), and $\mathrm{LiBF}_{4}(0.9 \mathrm{mg}, 0.01 \mathrm{mmol}, 5 \mathrm{~mol} \%$ ) in THF $(600 \mu \mathrm{L})$ under blue LED irradiation for $12 \mathrm{~h}$. The product 11caa was purified by flash chromatography on silica gel (100:0-95:5, hexane/EtOAc) (Scheme 3; $35.7 \mathrm{mg}, 0.11 \mathrm{mmol}, 54 \%$ isolated yield). Yellow oil. IR (neat) 699, 1091, 1154, 1247, 1493, 1734, $2956 \mathrm{~cm}^{-1}$. ${ }^{1} \mathbf{H}$ NMR (400 $\left.\mathrm{MHz} \mathrm{CDCl}_{3}\right) \delta 7.31-7.21(\mathrm{~m}, 7 \mathrm{H}), 7.17-7.14(\mathrm{~m}, 2 \mathrm{H}), 5.85(\mathrm{dd}, J=8.8,3.2 \mathrm{~Hz}, 1 \mathrm{H}), 3.56(\mathrm{~s}, 2 \mathrm{H})$, $1.92(\mathrm{dd}, J=14.8,8.8 \mathrm{~Hz}, 1 \mathrm{H}), 1.59$ (dd, $J=14.8,3.2 \mathrm{~Hz}, 1 \mathrm{H}), 0.89$ (s, 9H). ${ }^{13} \mathrm{C}$ NMR $(100.6 \mathrm{MHz}$, $\left.\mathrm{CDCl}_{3}\right) \delta 170.2,142.1,132.9,132.3,130.7,128.6,128.4,127.7,126.2,74.7,49.8,41.1,30.4,29.8$. HRMS-DART $(\mathrm{m} / \mathrm{z})$ : $\left[\mathrm{M}+\mathrm{NH}_{4}\right]^{+}$calcd for $\mathrm{C}_{20} \mathrm{H}_{27} \mathrm{ClNO}_{2}$, 348.1725; found, 348.1723.

\section{3,3-Dimethyl-1-phenylbutyl Undecanoate (11daa)}

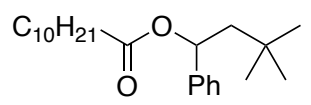

11daa

The reaction was carried out with $\mathbf{6 d}(111.8 \mathrm{mg}, 0.6 \mathrm{mmol}), \mathbf{2 a}(22.9 \mu 1,0.2 \mathrm{mmol}), \mathbf{3 a}(49.5 \mathrm{mg}$, $0.2 \mathrm{mmol}$ ), PTH1 (3.9 mg, $0.01 \mathrm{mmol}, 5 \mathrm{~mol} \%$ ), and LiBF 4 (0.9 mg, $0.01 \mathrm{mmol}, 5 \mathrm{~mol} \%$ ) in THF $(600 \mu \mathrm{L})$ under blue LED irradiation for $12 \mathrm{~h}$. The product 11daa was purified by flash chromatography on silica gel (100:0-95:5, hexane/EtOAc) (Scheme 3; $29.1 \mathrm{mg}, 0.08 \mathrm{mmol}, 42 \%$ isolated yield). Pale yellow oil. IR (neat) 698, 1162, 1366, 1466, 1736, 2854, 2924, $2954 \mathrm{~cm}^{-1} .{ }^{1} \mathbf{H}$ NMR (400 MHz, $\left.\mathrm{CDCl}_{3}\right) \delta 7.33-7.22(\mathrm{~m}, 5 \mathrm{H}), 5.86(\mathrm{dd}, J=9.2,3.2 \mathrm{~Hz}, 1 \mathrm{H}), 2.27(\mathrm{t}, J=7.6 \mathrm{~Hz}$, 2H), $1.95(\mathrm{dd}, J=14.8,9.2 \mathrm{~Hz}, 1 \mathrm{H}), 1.62-1.58(\mathrm{~m}, 2 \mathrm{H}), 1.24(\mathrm{br} \mathrm{s}, 15 \mathrm{H}), 0.95(\mathrm{~s}, 9 \mathrm{H}), 0.88(\mathrm{t}, J=$ $6.4 \mathrm{~Hz}, 3 \mathrm{H}) .{ }^{13} \mathbf{C}$ NMR $\left(100.6 \mathrm{MHz}, \mathrm{CDCl}_{3}\right) \delta 173.0,142.6,128.4,127.5,126.2,73.7,50.0,34.7$, $31.9,30.5,29.9,29.5,29.4,29.3,29.2,29.1,24.8,22.7,14.7$. HRMS-DART $(\mathrm{m} / z):\left[\mathrm{M}+\mathrm{NH}_{4}\right]^{+}$calcd for $\mathrm{C}_{23} \mathrm{H}_{42} \mathrm{NO}_{2}, 364.3210$; found, 364.3211 . 


\section{3,3-Dimethyl-1-phenylbutyl 5-Oxo-5-phenylpentanoate (11eaa)}

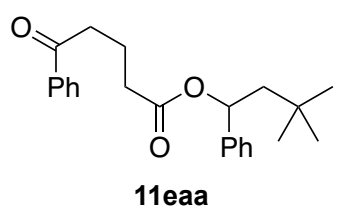

The reaction was carried out with $\mathbf{6 e}(115.3 \mathrm{mg}, 0.6 \mathrm{mmol}), \mathbf{2 a}(22.9 \mu 1,0.2 \mathrm{mmol}), \mathbf{3 a}(49.5 \mathrm{mg}$, $0.2 \mathrm{mmol}$ ), PTH1 (3.9 mg, $0.01 \mathrm{mmol}, 5 \mathrm{~mol} \%$ ), and $\mathrm{LiBF}_{4}(0.9 \mathrm{mg}, 0.01 \mathrm{mmol}, 5 \mathrm{~mol} \%)$ in THF $(600 \mu \mathrm{L})$ under blue LED irradiation for $12 \mathrm{~h}$. The product 11eaa was purified by flash chromatography on silica gel (100:0-90:10, hexane/EtOAc) (Scheme 3; $31.0 \mathrm{mg}, 0.09 \mathrm{mmol}, 44 \%$ isolated yield). Yellow oil. IR (neat) 699, 738, 1208, 1366, 1449, 1686, 1731, $2954 \mathrm{~cm}^{-1}$. ${ }^{1} \mathbf{H}$ NMR $\left(400 \mathrm{MHz}, \mathrm{CDCl}_{3}\right) \delta 7.90-7.88(\mathrm{~m}, 2 \mathrm{H}), 7.55(\mathrm{~m}, 1 \mathrm{H}), 7.46-7.42(\mathrm{~m}, 2 \mathrm{H}), 7.32-7.27(\mathrm{~m}, 4 \mathrm{H}), 7.24$ $(\mathrm{m}, 1 \mathrm{H}), 5.89(\mathrm{dd}, J=8.8,3.2 \mathrm{~Hz}, 1 \mathrm{H}), 2.97-2.94(\mathrm{~m}, 2 \mathrm{H}), 2.43(\mathrm{t}, J=7.2 \mathrm{~Hz}, 2 \mathrm{H}), 2.08-2.01(\mathrm{~m}$, 2H), $1.96(\mathrm{dd}, J=14.8,8.8 \mathrm{~Hz}, 1 \mathrm{H}), 1.61(\mathrm{dd}, J=14.8,3.2 \mathrm{~Hz} 1 \mathrm{H}), 0.94$ (s, 9H). ${ }^{13} \mathrm{C}$ NMR $(100.6$ $\left.\mathrm{MHz}_{2} \mathrm{CDCl}_{3}\right) \delta 199.4,172.5,142.4,136.8,133.0,128.5,128.4,128.0,127.7,126.3,74.0,49.9,37.3$, 33.7, 30.5, 29.9, 19.3. HRMS-DART $(\mathrm{m} / \mathrm{z})$ : $[\mathrm{M}+\mathrm{H}]^{+}$calcd for $\mathrm{C}_{23} \mathrm{H}_{29} \mathrm{O}_{3}, 353.2111$; found, 353.2114 .

\section{3,3-Dimethyl-1-phenylbutyl Tetrahydro-2H-pyran-4-carboxylate (11faa)}

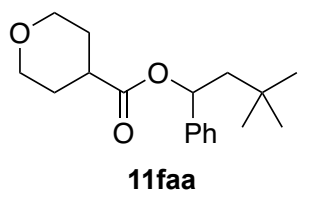

The reaction was carried out with $\mathbf{6 f}(78.1 \mathrm{mg}, 0.6 \mathrm{mmol}), \mathbf{2 a}(22.9 \mu 1,0.2 \mathrm{mmol}), \mathbf{3 a}(49.5 \mathrm{mg}$, $0.2 \mathrm{mmol}$ ), PTH1 (3.9 mg, $0.01 \mathrm{mmol}, 5 \mathrm{~mol} \%$ ), and $\mathrm{LiBF}_{4}(0.9 \mathrm{mg}, 0.01 \mathrm{mmol}, 5 \mathrm{~mol} \%$ ) in THF $(600 \mu \mathrm{L})$ under blue LED irradiation for $12 \mathrm{~h}$. The product 11 faa was purified by flash chromatography on silica gel (100:0-90:10, hexane/EtOAc) (Scheme 3; $30.8 \mathrm{mg}, 0.11 \mathrm{mmol}, 53 \%$ isolated yield). Yellow oil. IR (neat) 545, 698, 1040, 1132, 1167, 1728, 2843, $2953 \mathrm{~cm}^{-1}$. ${ }^{1} \mathbf{H}$ NMR $\left(400 \mathrm{MHz}, \mathrm{CDCl}_{3}\right) \delta 7.34-7.24(\mathrm{~m}, 5 \mathrm{H}), 5.87(\mathrm{dd}, J=9.2,2.4 \mathrm{~Hz}, 1 \mathrm{H}), 3.95-3.93(\mathrm{~m}, 2 \mathrm{H}), 3.41(\mathrm{t}, J$ $=11.2 \mathrm{~Hz}, 2 \mathrm{H}), 2.50(\mathrm{~m}, 1 \mathrm{H}), 1.96(\mathrm{dd}, J=14.8,9.2 \mathrm{~Hz}, 1 \mathrm{H}), 1.85-1.71(\mathrm{~m}, 4 \mathrm{H}), 1.61(\mathrm{dd}, J=14.8$, $2.4 \mathrm{~Hz}, 1 \mathrm{H}), 0.95(\mathrm{~s}, 9 \mathrm{H}) .{ }^{13} \mathbf{C}$ NMR $\left(100.6 \mathrm{MHz} \mathrm{CDCl}_{3}\right) \delta 173.5,142.5,128.5,127.7,126.1,74.1$, 67.1, 67.0, 50.0, 40.3, 30.5, 29.9, 28.5, 28.4. HRMS-DART $(\mathrm{m} / z):\left[\mathrm{M}+\mathrm{NH}_{4}\right]^{+}$calcd for $\mathrm{C}_{18} \mathrm{H}_{30} \mathrm{NO}_{3}$, 308.2220; found, 308.2221.

\section{3,3-Dimethyl-1-phenylbutyl 2,2,2-Trifluoroacetate (11gaa)}

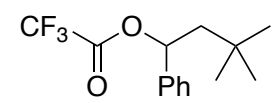

11 gaa

The reaction conditions were modified. The reaction was carried out with $\mathbf{6 g}(45.9 \mu 1,0.6 \mathrm{mmol})$, 2a (22.9 $\mu \mathrm{l}, 0.2 \mathrm{mmol}), 3 \mathbf{a}(49.5 \mathrm{mg}, 0.2 \mathrm{mmol})$, Molecular Sieves $3 \AA$ (50 mg), PTH1 (3.9 mg, 0.01 mmol, $5 \mathrm{~mol} \%)$, and $\mathrm{LiBF}_{4}(0.9 \mathrm{mg}, 0.01 \mathrm{mmol}, 5 \mathrm{~mol} \%)$ in THF (600 $\left.\mu \mathrm{L}\right)$ under blue LED irradiation for $12 \mathrm{~h}$. The product 11gaa was purified by flash chromatography on silica gel (100:0-99:1, 
hexane/EtOAc) (Scheme 3; $26.9 \mathrm{mg}, 0.10 \mathrm{mmol}, 49 \%$ isolated yield). Yellow oil. The spectrum data of product 11gaa was consistent with the literature. ${ }^{15}$

\section{1-(3,3-Dimethyl-1-phenylbutyl)pyrrolidin-2-one (12aaa)}

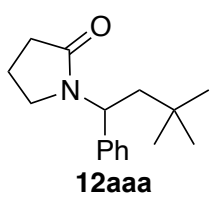

The reaction conditions were modified. The reaction was carried out with $7 \mathbf{a}(45.6 \mu 1,0.6 \mathrm{mmol})$, 2a (22.9 $\mu \mathrm{l}, 0.2 \mathrm{mmol}), 3 \mathbf{a}(49.5 \mathrm{mg}, 0.2 \mathrm{mmol})$, PTH1 (0.79 mg, $0.01 \mathrm{mmol}, 1 \mathrm{~mol} \%)$, and $\mathrm{LiBF}_{4}$ $(0.19 \mathrm{mg}, 0.002 \mathrm{mmol}, 1 \mathrm{~mol} \%)$ in $\mathrm{MeCN}(600 \mu \mathrm{L})$ under blue LED irradiation for $17 \mathrm{~h}$. The product 12aaa was purified by flash chromatography on silica gel (100:0-70:30, hexane/EtOAc) (Scheme 3; $28.5 \mathrm{mg}, 0.12 \mathrm{mmol}, 58 \%$ isolated yield). Pale yellow oil. IR (neat) 698, 1049, 1305, 1334, 1366, 1647, 2870, $2952 \mathrm{~cm}^{-1} .{ }^{1} \mathbf{H}$ NMR (400 MHz, $\left.\mathrm{CDCl}_{3}\right) \delta 7.34-7.22(\mathrm{~m}, 5 \mathrm{H}), 5.89(\mathrm{~d}, J=9.2 \mathrm{~Hz}, 1 \mathrm{H})$, 3.66-3.52 (m, 2H), 2.50-2.36 (m, 2H), 2.00-1.87 (m, 3H), $1.58(\mathrm{~m}, 1 \mathrm{H}), 0.97(\mathrm{~s}, 9 \mathrm{H}) .{ }^{13} \mathbf{C}$ NMR $\left(100.6 \mathrm{MHz}, \mathrm{CDCl}_{3}\right) \delta 171.7,143.4,128.3,127.1,125.9,76.9,55.3,51.3,31.5,30.6,30.0,22.8$. HRMS-DART $(\mathrm{m} / \mathrm{z})$ : $[\mathrm{M}+\mathrm{H}]^{+}$calcd for $\mathrm{C}_{16} \mathrm{H}_{24} \mathrm{NO}, 246.1852$; found, 246.1854.

\section{3-[4-(Benzyloxy)-3,3-dimethyl-1-phenylbutyl]oxazolidin-2-one (12bac)}

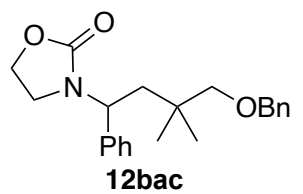

The reaction conditions were modified. The reaction was carried out with $7 \mathbf{b}(52.2 \mathrm{mg}, 0.6 \mathrm{mmol})$, 2a (22.9 $\mu \mathrm{l}, 0.2 \mathrm{mmol}), 3 \mathbf{c}(70.7 \mathrm{mg}, 0.2 \mathrm{mmol})$, PTH1 (0.79 mg, $0.01 \mathrm{mmol}, 1 \mathrm{~mol} \%)$, and $\mathrm{LiBF}_{4}$ $(0.19 \mathrm{mg}, 0.002 \mathrm{mmol}, 1 \mathrm{~mol} \%)$ in $\mathrm{MeCN}(600 \mu \mathrm{L})$ under blue LED irradiation for $24 \mathrm{~h}$. The product 12bac was purified by flash chromatography on silica gel (100:0-95:5, hexane/EtOAc) (Scheme 3; $36.8 \mathrm{mg}, 0.10 \mathrm{mmol}, 52 \%$ isolated yield). Pale yellow oil. IR (neat) 700, 760, 1097, 1248, 1420, 1737 , 2870, $2956 \mathrm{~cm}^{-1} .{ }^{1} \mathbf{H}$ NMR $\left(400 \mathrm{MHz}, \mathrm{CDCl}_{3}\right) \delta 7.35-7.20(\mathrm{~m}, 10 \mathrm{H}), 5.19(\mathrm{dd}, J=9.6,4.0 \mathrm{~Hz}, 1 \mathrm{H})$, 4.45 (s, 2H), 4.14-4.05 (m, 2H), $3.58(\mathrm{~m}, 1 \mathrm{H}), 3.27$ (d, J=8.8 Hz, 1H), 3.14-3.08 (m, 2H), 2.14 (dd, $J=14.4,9.6 \mathrm{~Hz}, 1 \mathrm{H}), 1.90(\mathrm{dd}, J=14.4,4.0 \mathrm{~Hz}, 1 \mathrm{H}), 1.01(\mathrm{~s}, 6 \mathrm{H}) .{ }^{13} \mathbf{C}$ NMR $\left(100.6 \mathrm{MHz}, \mathrm{CDCl}_{3}\right)$ $\delta 157.7,139.9,138.7,128.6,128.3,127.8,127.6,127.4,127.2,78.5,73.0,61.8,53.3,40.0,38.0,34.7$, 25.8, 24.9. HRMS-DART $(\mathrm{m} / \mathrm{z})$ : $[\mathrm{M}+\mathrm{H}]^{+}$calcd for $\mathrm{C}_{22} \mathrm{H}_{28} \mathrm{NO}_{3}, 354.2064$; found, 354.2064.

\section{$N$-(3,3-Dimethyl-1-phenylbutyl)- $N$-phenylacetamide (12caa)}

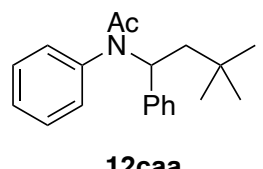

The reaction conditions were modified. The reaction was carried out with $7 \mathrm{c}(81.1 \mathrm{mg}, 0.6 \mathrm{mmol})$, 2a $(22.9 \mu \mathrm{l}, 0.2 \mathrm{mmol}), 3 \mathbf{3}(49.5 \mathrm{mg}, 0.2 \mathrm{mmol})$, Molecular Sieves $3 \AA$ (50 mg), PTH1 (3.9 mg, 0.01 mmol, $5 \mathrm{~mol} \%$ ), and $\mathrm{LiBF}_{4}(0.9 \mathrm{mg}, 0.01 \mathrm{mmol}, 5 \mathrm{~mol} \%)$ in DCM $(600 \mu \mathrm{L})$ under blue LED 
irradiation for $12 \mathrm{~h}$. The product 12caa was purified by flash chromatography on aluminium oxide (100:0, hexane/EtOAc) (Scheme 3; $30.7 \mathrm{mg}, 0.10 \mathrm{mmol}, 52 \%$ isolated yield). Pale yellow oil. IR (neat) 697, 749, 1235, 1274, 1373, 1596, 1671, $2952 \mathrm{~cm}^{-1} .{ }^{1} \mathbf{H} \mathbf{~ N M R}\left(400 \mathrm{MHz}, \mathrm{CDCl}_{3}\right) \delta 7.41-7.19$ $(\mathrm{m}, 7 \mathrm{H}), 6.97$ (t, $J=7.2 \mathrm{~Hz}, 1 \mathrm{H}), 6.59$ (d, $J=7.6 \mathrm{~Hz}, 2 \mathrm{H}), 6.17$ (d, $J=8.4 \mathrm{~Hz}, 1 \mathrm{H}), 2.02$ (dd, $J=14.4$, $9.2 \mathrm{~Hz}, 1 \mathrm{H}), 1.80(\mathrm{~s}, 3 \mathrm{H}), 1.62(\mathrm{~d}, J=14.4,1.2 \mathrm{~Hz}, 1 \mathrm{H}), 1.01(\mathrm{~s}, 9 \mathrm{H}) .{ }^{13} \mathbf{C ~ N M R}\left(100.6 \mathrm{MHz}, \mathrm{CDCl}_{3}\right)$ $\delta 159.5,149.2,143.6,128.8,128.1,127.0,126.5,122.5,121.0,73.7,50.6,30.7,30.0,16.5$. HRMSDART $(\mathrm{m} / \mathrm{z}):[\mathrm{M}+\mathrm{H}]^{+}$calcd for $\mathrm{C}_{20} \mathrm{H}_{26} \mathrm{NO}, 296.2009$; found, 296.2007.

\section{$N$-[4-(tert-Butyl)phenyl]-N-(3,3-dimethyl-1-phenylbutyl)acetamide (12daa)}

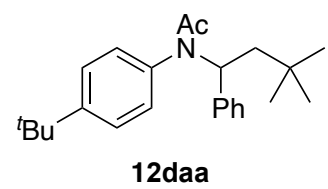

The reaction conditions were modified. The reaction was carried out with $7 \mathbf{d}$ (114.8 $\mathrm{mg}, 0.6 \mathrm{mmol})$, 2a (22.9 $\mu \mathrm{l}, 0.2 \mathrm{mmol}), 3 \mathrm{a}$ (49.5 mg, $0.2 \mathrm{mmol})$, Molecular Sieves $3 \AA$ (50 mg), PTH1 (3.9 mg, 0.01 mmol, $5 \mathrm{~mol} \%$ ), and $\mathrm{LiBF}_{4}(0.9 \mathrm{mg}, 0.01 \mathrm{mmol}, 5 \mathrm{~mol} \%)$ in DCM (600 $\left.\mu \mathrm{L}\right)$ under blue LED irradiation for $12 \mathrm{~h}$. The product $\mathbf{1 2 d a a}$ was purified by flash chromatography on aluminium oxide (100:0, hexane/EtOAc) (Scheme 3; $35.9 \mathrm{mg}, 0.10 \mathrm{mmol}, 51 \%$ isolated yield). Pale yellow oil. IR (neat) 698, 840, 1237, 1272, 1364, 1670, $2953 \mathrm{~cm}^{-1} .{ }^{1} \mathbf{H}$ NMR (400 MHz, $\left.\mathrm{CDCl}_{3}\right) \delta 7.39-7.30(\mathrm{~m}$, 4H), 7.24-7.20 (m, 3H), 6.55-6.51 (m, 2H), $6.17(\mathrm{dd}, J=9.2,2.8 \mathrm{~Hz}, 1 \mathrm{H}), 2.02(\mathrm{dd}, J=14.8,9.2 \mathrm{~Hz}$, $1 \mathrm{H}), 1.81(\mathrm{~s}, 3 \mathrm{H}), 1.61(\mathrm{dd}, J=14.8,2.8 \mathrm{~Hz}, 1 \mathrm{H}), 1.27(\mathrm{~s}, 9 \mathrm{H}), 1.01(\mathrm{~s}, 9 \mathrm{H}) .{ }^{13} \mathbf{C}$ NMR $(100.6 \mathrm{MHz}$, $\left.\mathrm{CDCl}_{3}\right) \delta 159.4,146.4,145.2,143.7,128.1,127.0,126.5,125.6,120.5,73.6,50.5,34.1,31.5,30.7$, 30.1, 16.5. HRMS-DART $(\mathrm{m} / \mathrm{z})$ : $[\mathrm{M}+\mathrm{H}]^{+}$calcd for $\mathrm{C}_{24} \mathrm{H}_{34} \mathrm{NO}, 352.2635$; found, 352.2635.

\section{$N$-(4-Bromophenyl)- $N$-(3,3-dimethyl-1-phenylbutyl)acetamide (12eaa)}

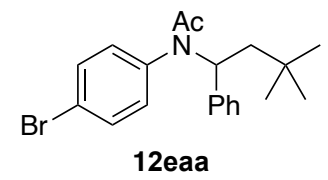

The reaction was carried out with 7e (128.4 mg, $0.6 \mathrm{mmol}), \mathbf{2 a}(22.9 \mu 1,0.2 \mathrm{mmol}), \mathbf{3 a}(49.5 \mathrm{mg}$, $0.2 \mathrm{mmol}$ ), PTH1 (0.79 mg, $0.002 \mathrm{mmol}, 1 \mathrm{~mol} \%$ ), and LiBF 4 (0.19 mg, $0.002 \mathrm{mmol}, 1 \mathrm{~mol} \%)$ in DCM $(600 \mu \mathrm{L})$ under blue LED irradiation for $12 \mathrm{~h}$. The product 12eaa was purified by flash chromatography on silica gel (100:0-95:5, hexane/EtOAc) (Scheme 3; $44.9 \mathrm{mg}, 0.12 \mathrm{mmol}, 60 \%$ isolated yield). Pale yellow oil. IR (neat) 698, 841, 962, 1235, 1278, 1374, 1482, 1668, $2953 \mathrm{~cm}^{-1}$. ${ }^{1} \mathbf{H}$ NMR (400 MHz, $\left.\mathrm{CDCl}_{3}\right) \delta 7.37-7.23(\mathrm{~m}, 7 \mathrm{H}), 6.47(\mathrm{~d}, J=8.0 \mathrm{~Hz}, 2 \mathrm{H}), 6.12(\mathrm{dd}, J=9.2,1.6 \mathrm{~Hz}$, 1H), $2.02(\mathrm{dd}, J=14.4,9.2 \mathrm{~Hz}, 1 \mathrm{H}), 1.79(\mathrm{~s}, 3 \mathrm{H}), 1.61(\mathrm{dd}, J=1.8,14.5 \mathrm{~Hz}, 1 \mathrm{H}), 1.00(\mathrm{~s}, 9 \mathrm{H}) .{ }^{13} \mathbf{C}$ NMR (100.6 MHz, $\left.\mathrm{CDCl}_{3}\right) \delta 160.0,148.3,143.3,131.7,128.2,127.1,126.4,122.8,115.4,74.1,50.5$, 30.7, 30.0, 16.5. HRMS-DART $(\mathrm{m} / \mathrm{z})$ : $[\mathrm{M}+\mathrm{H}]^{+}$calcd for $\mathrm{C}_{20} \mathrm{H}_{25} \mathrm{BrNO}, 374.1114$; found, 374.1106 . 


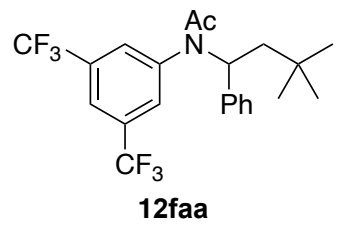

The reaction conditions were modified. The reaction was carried out with $7 \mathbf{f}(162.7 \mathrm{mg}, 0.6 \mathrm{mmol})$, 2a (22.9 $\mu 1,0.2 \mathrm{mmol}), 3 a(49.5 \mathrm{mg}, 0.2 \mathrm{mmol})$, Molecular Sieves $3 \AA$ (50 mg), PTH1 (0.79 mg, $0.002 \mathrm{mmol}, 1 \mathrm{~mol} \%)$, and $\mathrm{LiBF}_{4}(0.19 \mathrm{mg}, 0.002 \mathrm{mmol}, 1 \mathrm{~mol} \%)$ in DCM (600 $\left.\mu \mathrm{L}\right)$ under blue LED irradiation for $12 \mathrm{~h}$. The product 12 faa was purified by flash chromatography on aluminium oxide (100:0, hexane/EtOAc) (Scheme 3; $49.2 \mathrm{mg}, 0.11 \mathrm{mmol}, 57 \%$ isolated yield). Pale yellow oil. IR (neat) 697, 1128, 1169, 1273, 1375, 1666, $2956 \mathrm{~cm}^{-1} .{ }^{1} \mathbf{H}$ NMR (400 MHz, $\left.\mathrm{CDCl}_{3}\right) \delta 7.49$ (br s, 1H), 7.36-7.28 (m, 5H), $7.00(\mathrm{~s}, 2 \mathrm{H}), 6.10(\mathrm{dd}, J=9.2,2.8 \mathrm{~Hz}, 1 \mathrm{H}), 2.05(\mathrm{dd}, J=14.8,9.2 \mathrm{~Hz}, 1 \mathrm{H}), 1.84$ $(\mathrm{s}, 3 \mathrm{H}), 1.63(\mathrm{dd}, J=14.8,2.8 \mathrm{~Hz}, 1 \mathrm{H}), 1.01(\mathrm{~s}, 9 \mathrm{H}) .{ }^{13} \mathbf{C ~ N M R}\left(100.6 \mathrm{MHz}, \mathrm{CDCl}_{3}\right) \delta 161.3,150.6$, $142.8,132.2\left(\mathrm{q},{ }^{2} J_{\mathrm{C}-\mathrm{F}}=33.1 \mathrm{~Hz}\right), 128.4,127.4,126.4,123.3\left(\mathrm{q},{ }^{1} J_{\mathrm{C}-\mathrm{F}}=272.7 \mathrm{~Hz}\right), 121.3\left(\mathrm{~d},{ }^{3} J_{\mathrm{C}-\mathrm{F}}=\right.$ $3.5 \mathrm{~Hz}$ ), 116.2 (quin, ${ }^{3} J_{\mathrm{C}-\mathrm{F}}=4.0 \mathrm{~Hz}$ ), 75.1, 50.4, 30.7, 30.0, 16.8. HRMS-DART $(\mathrm{m} / \mathrm{z}):[\mathrm{M}+\mathrm{H}]^{+}$ calcd for $\mathrm{C}_{22} \mathrm{H}_{24} \mathrm{~F}_{6} \mathrm{NO}, 432.1757$; found, 432.1756 .

\section{4-Fluoro-2,2-dimethyl-4-phenylbutyl Acetate (13aab)}

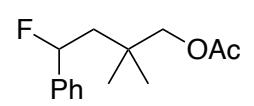

$13 \mathrm{aab}$

The reaction was carried out with triethylamine trihydrofluoride $\mathbf{8 a}(97.7 \mu 1,0.6 \mathrm{mmol}), \mathbf{2 a}(22.9$ $\mu$ l, $0.2 \mathrm{mmol}$ ), 3b (61.1 mg, $0.2 \mathrm{mmol}$ ), PTH1 (3.9 mg, $0.01 \mathrm{mmol}, 5 \mathrm{~mol} \%$ ), and $\mathrm{LiBF}_{4}(0.9 \mathrm{mg}$, $0.01 \mathrm{mmol}, 5 \mathrm{~mol} \%)$ in DCM $(600 \mu \mathrm{L})$ under blue LED irradiation for $12 \mathrm{~h}$. The product 13aab was purified by flash chromatography on silica gel (100:0-70:30, hexane/EtOAc) (Scheme 3; $27.6 \mathrm{mg}$, $0.12 \mathrm{mmol}, 58 \%$ isolated yield). Pale yellow oil. IR (neat) 699, 746, 1038, 1240, 1377, 1735, 2962 $\mathrm{cm}^{-1} .{ }^{1} \mathbf{H}$ NMR $\left(400 \mathrm{MHz}, \mathrm{CDCl}_{3}\right) \delta 7.39-7.31(\mathrm{~m}, 5 \mathrm{H}), 5.61(\mathrm{dd}, J=48.8,9.6 \mathrm{~Hz}, 1 \mathrm{H}), 3.96-3.87$ (m, 2H), 2.09-1.98 (m, 4H), $1.68(\mathrm{dd}, J=40.0,15.2 \mathrm{~Hz}, 1 \mathrm{H}), 1.09$ (s, 3H), 1.07 (s, 3H). ${ }^{13} \mathbf{C}$ NMR $\left(100.6 \mathrm{MHz} \mathrm{CDCl}_{3}\right) \delta 171.1,141.2\left(\mathrm{~d},{ }^{2} J_{\mathrm{C}-\mathrm{F}}=20.0 \mathrm{~Hz}\right), 128.5,128.2\left(\mathrm{~d},{ }^{4} J_{\mathrm{C}-\mathrm{F}}=2.0 \mathrm{~Hz}\right), 125.3(\mathrm{~d}$, $\left.{ }^{3} J_{\mathrm{C}-\mathrm{F}}=6.7 \mathrm{~Hz}\right), 92.1\left(\mathrm{~d},{ }^{1} J_{\mathrm{C}-\mathrm{F}}=171.1 \mathrm{~Hz}\right), 72.4\left(\mathrm{~d},{ }^{4} J_{\mathrm{C}-\mathrm{F}}=1.9 \mathrm{~Hz}\right), 46.4\left(\mathrm{~d},{ }^{2} J_{\mathrm{C}-\mathrm{F}}=22.7 \mathrm{~Hz}\right), 33.7$, $24.8\left(\mathrm{~d},{ }^{3} J_{\mathrm{C}-\mathrm{F}}=1.9 \mathrm{~Hz}\right), 24.7,20.9 .{ }^{19} \mathbf{F}$ NMR $\left(376.5 \mathrm{MHz}, \mathrm{CDCl}_{3}\right) \delta-172.7(\mathrm{ddd}, J=48.6,41.8$, $17.7 \mathrm{~Hz})$. HRMS-DART $(\mathrm{m} / \mathrm{z})$ : $\left[\mathrm{M}+\mathrm{NH}_{4}\right]^{+}$calcd for $\mathrm{C}_{14} \mathrm{H}_{23} \mathrm{FNO}_{2}, 256.1707$; found, 256.1708 .

\section{[1-Fluoro-2-(1-methylcyclohexyl)ethyl]benzene (13aaf)}

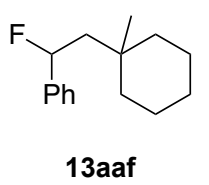

The reaction was carried out with triethylamine trihydrofluoride 8a (97.7 $\mu 1,0.6 \mathrm{mmol}), \mathbf{2 a}(22.9$ $\mu \mathrm{l}, 0.2 \mathrm{mmol}$ ), 3f (57.5 mg, $0.2 \mathrm{mmol}$ ), PTH1 (3.9 mg, $0.01 \mathrm{mmol}, 5 \mathrm{~mol} \%$ ), and $\mathrm{LiBF}_{4}(0.9 \mathrm{mg}, 0.01$ mmol, $5 \mathrm{~mol} \%)$ in DCM $(600 \mu \mathrm{L})$ under blue LED irradiation for $12 \mathrm{~h}$. The product 13aaf was purified by flash chromatography on silica gel (100:0-90:10, hexane/EtOAc) (Scheme 3; $25.1 \mathrm{mg}$, 
$0.11 \mathrm{mmol}, 57 \%$ isolated yield). Colorless oil. IR (neat) 697, 759, 963, 1059, 1453, 2851, $2923 \mathrm{~cm}^{-}$ ${ }^{1} .{ }^{1} \mathbf{H}$ NMR $\left(400 \mathrm{MHz}, \mathrm{CDCl}_{3}\right) \delta 7.38-7.28(\mathrm{~m}, 5 \mathrm{H}), 5.62(\mathrm{ddd}, J=48.8,9.6,2.0 \mathrm{~Hz}, 1 \mathrm{H}), 2.00(\mathrm{~m}$, $1 \mathrm{H}), 1.61(\mathrm{~m}, 1 \mathrm{H}), 1.50-1.31(\mathrm{~m}, 10 \mathrm{H}), 1.05(\mathrm{~s}, 3 \mathrm{H}) .{ }^{13} \mathbf{C} \mathbf{N M R}\left(100.6 \mathrm{MHz}, \mathrm{CDCl}_{3}\right) \delta 142.1\left(\mathrm{~d},{ }^{2} J_{\mathrm{C}-\mathrm{F}}\right.$ $=20.2 \mathrm{~Hz}), 128.4,127.9\left(\mathrm{~d},{ }^{4} J_{\mathrm{C}-\mathrm{F}}=1.5 \mathrm{~Hz}\right), 125.3\left(\mathrm{~d},{ }^{3} J_{\mathrm{C}-\mathrm{F}}=6.7 \mathrm{~Hz}\right), 92.2\left(\mathrm{~d},{ }^{1} J_{\mathrm{C}-\mathrm{F}}=170.6 \mathrm{~Hz}\right)$, 49.6, 38.4 (d, $\left.{ }^{3} J_{\mathrm{C}-\mathrm{F}}=1.8 \mathrm{~Hz}\right), 38.1,32.7,26.3(\times 2 \mathrm{C}), 25.5,22.0\left(\mathrm{~d},{ }^{2} J_{\mathrm{C}-\mathrm{F}}=2.9 \mathrm{~Hz}\right) .{ }^{19} \mathbf{F} \mathbf{~ N M R}(376.5$ MHz, $\left.\mathrm{CDCl}_{3}\right) \delta-171.7(\mathrm{dt}, J=44.1,20.0 \mathrm{~Hz})$. HRMS-DART $(\mathrm{m} / z):\left[\mathrm{M}+\mathrm{NH}_{4}\right]^{+}$calcd for $\mathrm{C}_{15} \mathrm{H}_{25} \mathrm{FN}$, 238.1966; found, 238.1966 .

\section{tert-Butyl 4-(2-Fluoro-2-phenylethyl)-4-methylpiperidine-1-carboxylate (13aag)}

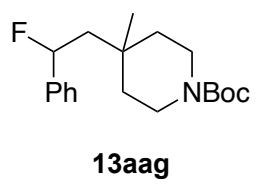

The reaction was carried out with triethylamine trihydrofluoride $\mathbf{8 a}(97.7 \mu 1,0.6 \mathrm{mmol}), \mathbf{2 a}(22.9$

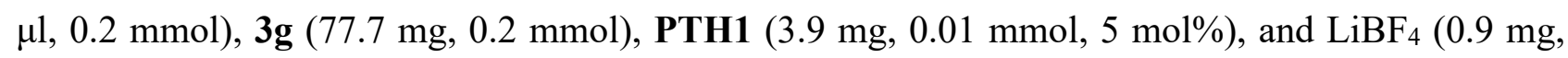
$0.01 \mathrm{mmol}, 5 \mathrm{~mol} \%)$ in DCM $(600 \mu \mathrm{L})$ under blue LED irradiation for $12 \mathrm{~h}$. The product 13 aag was purified by flash chromatography on silica gel (100:0-90:10, hexane/EtOAc) (Scheme 3; 36.6 mg, $0.11 \mathrm{mmol}, 57 \%$ isolated yield). Pale yellow oil. IR (neat) 698, 764, 1159, 1246, 1364, 1421, 1686, $2928 \mathrm{~cm}^{-1} .{ }^{1} \mathbf{H}$ NMR $\left(400 \mathrm{MHz}, \mathrm{CDCl}_{3}\right) \delta 7.39-7.30(\mathrm{~m}, 5 \mathrm{H}), 5.64(\mathrm{ddd}, J=48.8,10.0,2.0 \mathrm{~Hz}, 1 \mathrm{H})$, $3.58-3.53(\mathrm{~m}, 2 \mathrm{H}), 3.30-3.24(\mathrm{~m}, 2 \mathrm{H}), 2.08(\mathrm{~m}, 1 \mathrm{H}), 1.67-1.53(\mathrm{~m}, 2 \mathrm{H}), 1.48-1.40(\mathrm{~m}, 12 \mathrm{H}), 1.13$ $(\mathrm{s}, 3 \mathrm{H}) .{ }^{13} \mathrm{C}$ NMR $\left(100.6 \mathrm{MHz}, \mathrm{CDCl}_{3}\right) \delta 154.9,141.4\left(\mathrm{~d},{ }^{2} J_{\mathrm{C}-\mathrm{F}}=20.0 \mathrm{~Hz}\right), 128.5,128.1\left(\mathrm{~d},{ }^{4} J_{\mathrm{C}-\mathrm{F}}=\right.$ $1.8 \mathrm{~Hz}), 125.2\left(\mathrm{~d},{ }^{3} J_{\mathrm{C}-\mathrm{F}}=6.6 \mathrm{~Hz}\right), 91.8\left(\mathrm{~d},{ }^{1} J_{\mathrm{C}-\mathrm{F}}=170.9 \mathrm{~Hz}\right), 79.3,49.2\left(\mathrm{~d}, J_{\mathrm{C}-\mathrm{F}}={ }^{2} 22.5 \mathrm{~Hz}\right), 39.5$, 37.2, 31.4, 28.4, $23.8\left(\mathrm{~d},{ }^{3} J_{\mathrm{C}-\mathrm{F}}=1.0 \mathrm{~Hz}\right) .{ }^{19} \mathbf{F}$ NMR (376.5 MHz, $\left.\mathrm{CDCl}_{3}\right) \delta-172.2$ (br s). HRMSDART $(\mathrm{m} / \mathrm{z}):[\mathrm{M}+\mathrm{H}]^{+}$calcd for $\mathrm{C}_{19} \mathrm{H}_{29} \mathrm{FNO}_{2}, 322.2177$; found, 322.2163 .

\section{4-(2-Fluoro-2-phenylethyl)-4-methyltetrahydro-2H-pyran (13aah)}

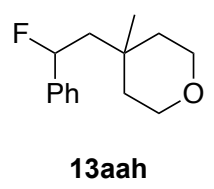

The reaction was carried out with triethylamine trihydrofluoride $\mathbf{8 a}(97.7 \mu 1,0.6 \mathrm{mmol}), \mathbf{2 a}(22.9$ $\mu$ l, $0.2 \mathrm{mmol}$ ), 3h (57.9 mg, $0.2 \mathrm{mmol}$ ), PTH1 (3.9 mg, $0.01 \mathrm{mmol}, 5 \mathrm{~mol} \%$ ), and $\mathrm{LiBF}_{4}(0.9 \mathrm{mg}$, $0.01 \mathrm{mmol}, 5 \mathrm{~mol} \%)$ in DCM $(600 \mu \mathrm{L})$ under blue LED irradiation for $12 \mathrm{~h}$. The product 13aah was purified by flash chromatography on silica gel (100:0-60:40, hexane/EtOAc) (Scheme 3; $25.8 \mathrm{mg}$, $0.12 \mathrm{mmol}, 58 \%$ isolated yield). Pale yellow oil. IR (neat) 540, 698, 763, 1108, 1454, 2850, 2923 $\mathrm{cm}^{-1} .{ }^{1} \mathbf{H}$ NMR $\left(400 \mathrm{MHz}, \mathrm{CDCl}_{3}\right) \delta 7.40-7.30(\mathrm{~m}, 5 \mathrm{H}), 5.65(\mathrm{dd}, J=48.8,10.0 \mathrm{~Hz}, 1 \mathrm{H}), 3.77-3.64$ (m, 4H), $2.12(\mathrm{~m}, 1 \mathrm{H}), 1.73-1.39(\mathrm{~m}, 5 \mathrm{H}), 1.18(\mathrm{~s}, 3 \mathrm{H}) .{ }^{13} \mathbf{C} \mathbf{N M R}\left(100.6 \mathrm{MHz}, \mathrm{CDCl}_{3}\right) \delta 141.4(\mathrm{~d}$, $\left.{ }^{2} J_{\mathrm{C}-\mathrm{F}}=20.2 \mathrm{~Hz}\right), 128.5,128.1\left(\mathrm{~d},{ }^{4} J_{\mathrm{C}-\mathrm{F}}=1.9 \mathrm{~Hz}\right), 125.2\left(\mathrm{~d},{ }^{3} J_{\mathrm{C}-\mathrm{F}}=6.6 \mathrm{~Hz}\right), 91.9\left(\mathrm{~d},{ }^{1} J_{\mathrm{C}-\mathrm{F}}=170.7\right.$ $\mathrm{Hz}), 63.8\left(\mathrm{~d},{ }^{3} J_{\mathrm{C}-\mathrm{F}}=12.6 \mathrm{~Hz}\right), 49.9\left(\mathrm{~d},{ }^{2} J_{\mathrm{C}-\mathrm{F}}=22.4 \mathrm{~Hz}\right), 38.1(\times 2 \mathrm{C}), 38.0\left(\mathrm{~d},{ }^{4} J_{\mathrm{C}-\mathrm{F}}=2.0 \mathrm{~Hz}\right), 30.7$, 24.1. ${ }^{19}$ F NMR $\left(376.5 \mathrm{MHz}, \mathrm{CDCl}_{3}\right) \delta-172.2(\mathrm{ddd}, J=48.9,41.4,18.4 \mathrm{~Hz})$. HRMS-DART $(\mathrm{m} / \mathrm{z})$ : $\left[\mathrm{M}+\mathrm{NH}_{4}\right]^{+}$calcd for $\mathrm{C}_{14} \mathrm{H}_{23} \mathrm{FNO}, 240.1758$; found, 240.1755 . 


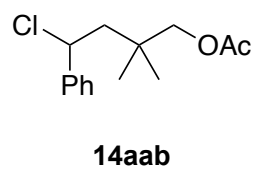

The reaction was carried out with collidine $\bullet \mathrm{HCl}$ 9a $(94.6 \mathrm{mg}, 0.6 \mathrm{mmol}), \mathbf{2 a}(22.9 \mu \mathrm{l}, 0.2 \mathrm{mmol})$, 3b (91.6 mg, $0.3 \mathrm{mmol}$ ), PTH1 (3.9 mg, $0.01 \mathrm{mmol}, 5 \mathrm{~mol} \%$ ), and $\mathrm{LiBF}_{4}$ (0.9 mg, $0.01 \mathrm{mmol}, 5$ mol\%) in DCM $(600 \mu \mathrm{L})$ under blue LED irradiation for $12 \mathrm{~h}$. The product $14 \mathbf{a a b}$ was purified by flash chromatography on silica gel (100:0-90:10, hexane/EtOAc) (Scheme 3; $27.5 \mathrm{mg}, 0.11 \mathrm{mmol}$, $54 \%$ isolated yield). Pale yellow oil. IR (neat) 699, 1038, 1237, 1377, 1455, 1736, $2963 \mathrm{~cm}^{-1} .{ }^{1} \mathbf{H}$ NMR (400 MHz, $\left.\mathrm{CDCl}_{3}\right) \delta 7.40-7.27(\mathrm{~m}, 5 \mathrm{H}), 4.99(\mathrm{t}, J=6.8 \mathrm{~Hz}, 1 \mathrm{H}), 3.79-3.69(\mathrm{~m}, 2 \mathrm{H}), 2.30$ $2.18(\mathrm{~m}, 2 \mathrm{H}), 2.05(\mathrm{~s}, 3 \mathrm{H}), 0.97(\mathrm{~s}, 3 \mathrm{H}), 0.92(\mathrm{~s}, 3 \mathrm{H}) .{ }^{13} \mathbf{C}$ NMR (100.6 MHz, $\left.\mathrm{CDCl}_{3}\right) \delta 170.9,142.9$, 128.7, 128.3, 126.9, 71.9, 60.1, 48.3, 34.4, 24.9, 24.7, 20.9. HRMS-DART $(\mathrm{m} / \mathrm{z}):\left[\mathrm{M}+\mathrm{NH}_{4}\right]^{+}$calcd for $\mathrm{C}_{14} \mathrm{H}_{23} \mathrm{ClNO}_{2}, 272.1412$; found, 272.1396 .

\section{[1-Chloro-2-(1-methylcyclohexyl)ethyl]benzene (14aaf)}

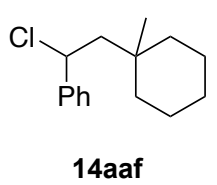

The reaction was carried out with collidine $\bullet \mathrm{HCl}$ 9a $(94.6 \mathrm{mg}, 0.6 \mathrm{mmol}), \mathbf{2 a}(22.9 \mu \mathrm{l}, 0.2 \mathrm{mmol})$, 3f (86.2 mg, $0.3 \mathrm{mmol}$ ), PTH1 (3.9 mg, $0.01 \mathrm{mmol}, 5 \mathrm{~mol} \%$ ), and $\mathrm{LiBF}_{4}$ (0.9 mg, $0.01 \mathrm{mmol}, 5$ mol\%) in DCM $(600 \mu \mathrm{L})$ under blue LED irradiation for $12 \mathrm{~h}$. The product 14aaf was purified by flash chromatography on silica gel (100:0, hexane/EtOAc) (Scheme 3; $25.5 \mathrm{mg}, 0.11 \mathrm{mmol}, 54 \%$ isolated yield). Pale yellow oil. IR (neat) 696, 729, 764, 1454, 2850, $2924 \mathrm{~cm}^{-1}$. ${ }^{1} \mathbf{H}$ NMR (400 MHz, $\left.\mathrm{CDCl}_{3}\right) \delta 7.41-7.27(\mathrm{~m}, 5 \mathrm{H}), 5.03(\mathrm{t}, J=6.8 \mathrm{~Hz}, 1 \mathrm{H}), 2.24(\mathrm{dd}, J=14.8,6.8 \mathrm{~Hz}, 1 \mathrm{H}), 2.14(\mathrm{dd}, J=$ 14.8, $6.8 \mathrm{~Hz}, 1 \mathrm{H}), 1.50-1.29(\mathrm{~m}, 8 \mathrm{H}), 1.19-1.09(\mathrm{~m}, 2 \mathrm{H}), 0.88$ (s, 3H). ${ }^{13} \mathrm{C}$ NMR (100.6 MHz, $\left.\mathrm{CDCl}_{3}\right) \delta 143.7,128.6,128.1,127.0,60.5,38.2,38.1,33.7,26.2,21.9,21.8$. HRMS-DART $(\mathrm{m} / z)$ : $\left[\mathrm{M}+\mathrm{NH}_{4}\right]^{+}$calcd for $\mathrm{C}_{15} \mathrm{H}_{25} \mathrm{ClN}, 254.1670$; found, 254.1658.

tert-Butyl 4-(2-chloro-2-phenylethyl)-4-methylpiperidine-1-carboxylate (14aag)

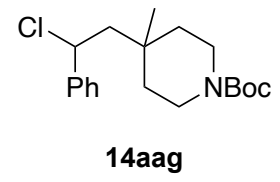

The reaction was carried out with collidine $\bullet \mathrm{HCl}$ 9a $(94.6 \mathrm{mg}, 0.6 \mathrm{mmol}), \mathbf{2 a}(22.9 \mu \mathrm{l}, 0.2 \mathrm{mmol})$, $3 g$ (116.5 mg, $0.3 \mathrm{mmol}$ ), PTH1 (3.9 mg, $0.01 \mathrm{mmol}, 5 \mathrm{~mol} \%$ ), and LiBF 4 (0.9 mg, $0.01 \mathrm{mmol}, 5$ mol\%) in DCM $(600 \mu \mathrm{L})$ under blue LED irradiation for $12 \mathrm{~h}$. Reaction was carried out with collidine $\cdot \mathrm{HCl}(0.6 \mathrm{mmol}), \mathbf{2 a}(0.2 \mathrm{mmol}), \mathbf{3 g}(0.3 \mathrm{mmol})$. The product 14aag was purified by flash chromatography on silica gel (100:0-90:10, hexane/EtOAc) (Scheme 3; $36.5 \mathrm{mg}, 0.11 \mathrm{mmol}, 54 \%$ isolated yield). Colorless oil. IR (neat) 699, 1162, 1248, 1423, 1689, 2871, 2926, $2972 \mathrm{~cm}^{-1} .{ }^{1} \mathbf{H}$ NMR (400 MHz, $\left.\mathrm{CDCl}_{3}\right) \delta 7.40-7.27(\mathrm{~m}, 5 \mathrm{H}), 5.02(\mathrm{t}, J=6.8 \mathrm{~Hz}, 1 \mathrm{H}), 3.60-3.44(\mathrm{~m}, 2 \mathrm{H}), 3.24-$ $3.08(\mathrm{~m}, 2 \mathrm{H}), 2.27(\mathrm{dd}, J=14.8,6.8 \mathrm{~Hz}, 1 \mathrm{H}), 2.15(\mathrm{dd}, J=14.8,6.8 \mathrm{~Hz}, 1 \mathrm{H}), 1.54(\mathrm{~m}, 1 \mathrm{H}), 1.44$ $1.38(\mathrm{~m}, 10 \mathrm{H}), 1.24(\mathrm{~m}, 1 \mathrm{H}), 1.14(\mathrm{~m}, 1 \mathrm{H}), 0.99(\mathrm{~s}, 3 \mathrm{H}) .{ }^{13} \mathbf{C} \mathbf{N M R}\left(100.6 \mathrm{MHz}, \mathrm{CDCl}_{3}\right) \delta 154.9$, 
143.2, 128.8, 128.3, 126.9, 79.3, 59.9, 51.4, 39.7, 37.1, 32.3, 28.4, 23.4. HRMS-DART $(\mathrm{m} / \mathrm{z})$ : $[\mathrm{M}+\mathrm{H}]^{+}$calcd for $\mathrm{C}_{19} \mathrm{H}_{29} \mathrm{ClNO}_{2}, 338.1881$; found, 338.1882 .

\section{4-(2-Chloro-2-phenylethyl)-4-methyltetrahydro-2H-pyran (14aah)}

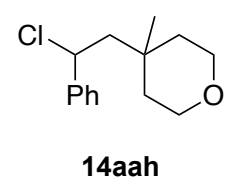

The reaction was carried out with collidine $\cdot \mathrm{HCl}$ 9a $(94.6 \mathrm{mg}, 0.6 \mathrm{mmol}), \mathbf{2 a}(22.9 \mu \mathrm{l}, 0.2 \mathrm{mmol})$, 3h (86.8 mg, $0.3 \mathrm{mmol}$ ), PTH1 (3.9 mg, $0.01 \mathrm{mmol}, 5 \mathrm{~mol} \%$ ), and $\mathrm{LiBF}_{4}$ (0.9 mg, $0.01 \mathrm{mmol}, 5$ mol\%) in DCM $(600 \mu \mathrm{L})$ under blue LED irradiation for $12 \mathrm{~h}$. Reaction was carried out with collidine $\cdot \mathrm{HCl}(0.6 \mathrm{mmol}), \mathbf{2 a}(0.2 \mathrm{mmol}), \mathbf{3 h}(0.3 \mathrm{mmol})$. The product $\mathbf{1 4 a a h}$ was purified by flash chromatography on silica gel (100:0-95:5, hexane/EtOAc) (Scheme 3; $24.8 \mathrm{mg}, 0.10 \mathrm{mmol}, 52 \%$ isolated yield). Pale yellow oil. IR (neat) 699, 1107, 1224, 1455, 2851, 2922, $2955 \mathrm{~cm}^{-1} .{ }^{1} \mathbf{H}$ NMR $\left(400 \mathrm{MHz}, \mathrm{CDCl}_{3}\right) \delta 7.41-7.27(\mathrm{~m}, 5 \mathrm{H}), 5.04(\mathrm{tt}, J=6.8 \mathrm{~Hz}, 1 \mathrm{H}), 3.73(\mathrm{~m}, 1 \mathrm{H}), 3.65-3.50(\mathrm{~m}, 3 \mathrm{H})$, $2.30(\mathrm{dd}, J=14.8,6.8 \mathrm{~Hz}, 1 \mathrm{H}), 2.18(\mathrm{dd}, J=14.8,6.8 \mathrm{~Hz}, 1 \mathrm{H}), 1.66(\mathrm{~m}, 1 \mathrm{H}), 1.46-1.32(\mathrm{~m}, 2 \mathrm{H})$, $1.14(\mathrm{~m}, 1 \mathrm{H}), 1.05(\mathrm{~s}, 3 \mathrm{H}) .{ }^{13} \mathbf{C}$ NMR $\left(100.6 \mathrm{MHz} \mathrm{CDCl}_{3}\right) \delta 143.2,128.7,128.3,126.9,63.7,63.6$, 59.8, 52.2, 38.1, 37.9, 31.6, 23.6. HRMS-DART $(\mathrm{m} / \mathrm{z})$ : $\left[\mathrm{M}+\mathrm{NH}_{4}\right]^{+}$calcd for $\mathrm{C}_{14} \mathrm{H}_{23} \mathrm{ClNO}, 256.1463$; found, 256.1474 . 


\section{- UV-Vis Spectra of Reaction Mixtures}

$\mathrm{UV}-\mathrm{Vis}$ spectra of the reation mixture were measured under identical concentration to the catalytic reaction conditions [3a $(0.2 \mathrm{mmol})$, PTH1 (1 or $10 \mathrm{~mol} \%)$ and $\mathrm{LiBF}_{4}(1$ or $10 \mathrm{~mol} \%)$ in acetonitrile $(600 \mu \mathrm{l})$ solution]. In Figure S2 (Scheme 4A in manuscript), PTH1 (1 mol\%) and LiBF (1 mol\%) were used. In Figure S3, PTH1 (10 mol\%) and LiBF (10 mol\%) were used.

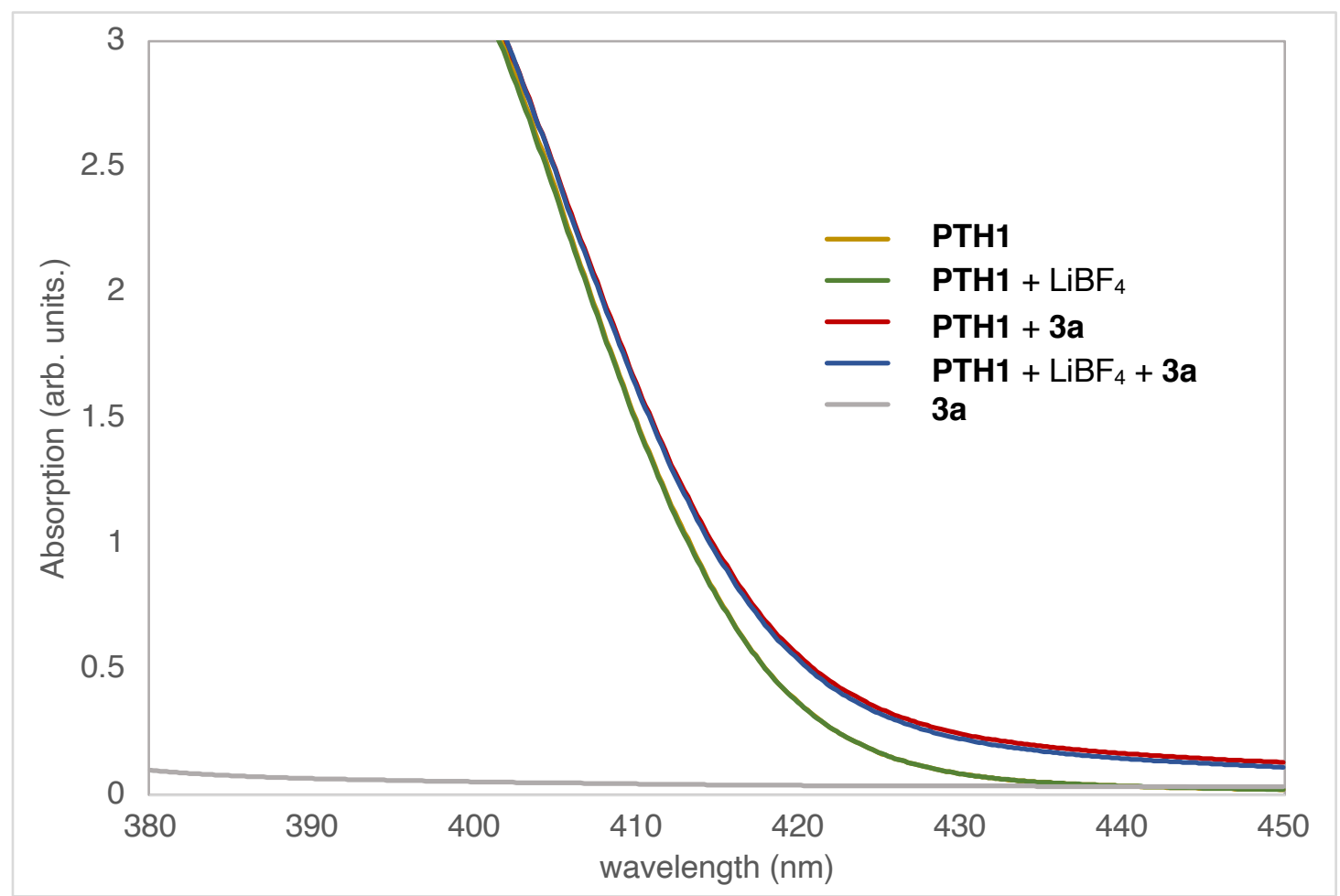

Figure S2. UV-Vis spectra of the reaction mixture [PTH1 (1 mol\%) and $\left.\mathrm{LiBF}_{4}(1 \mathrm{~mol} \%)\right]$.

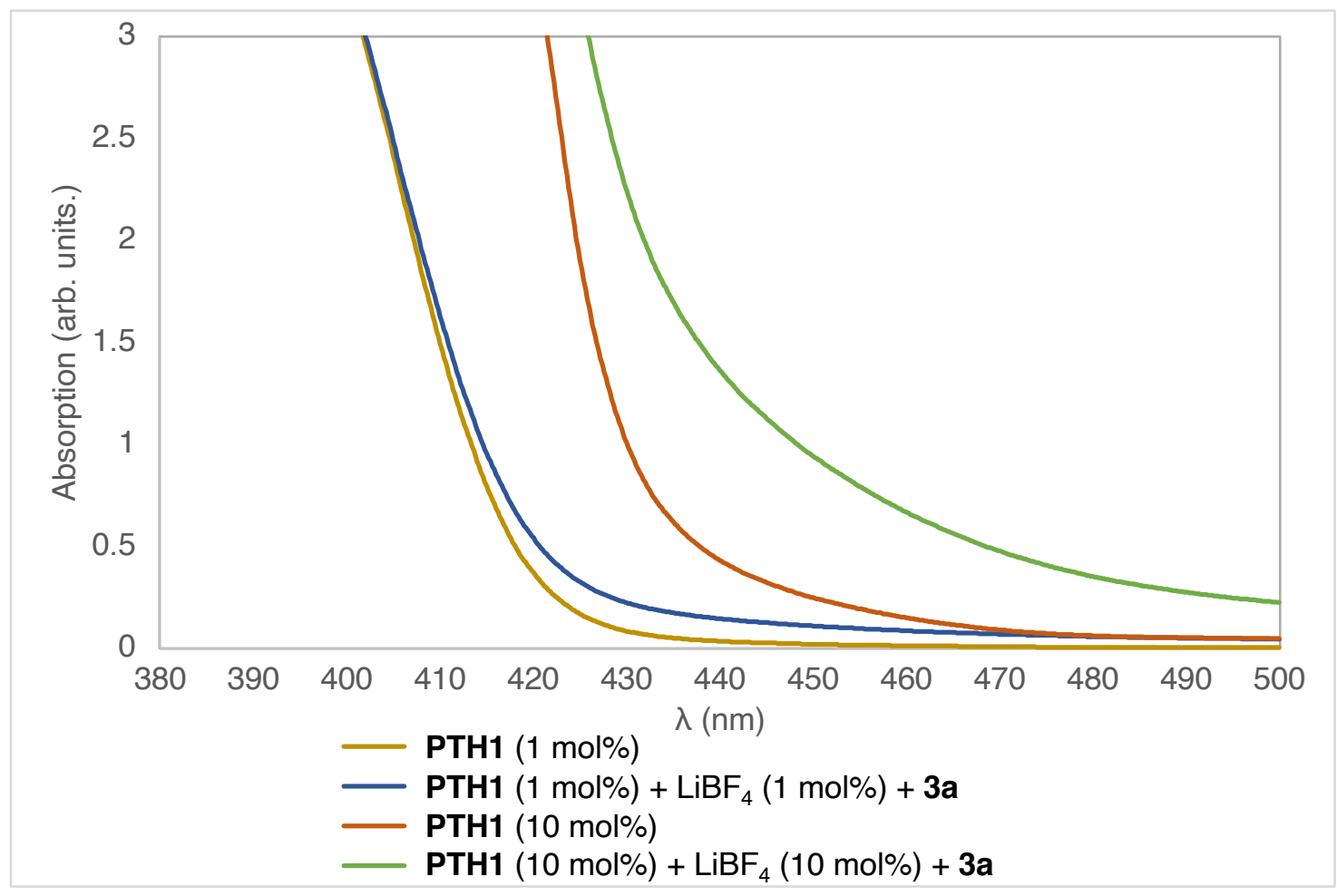

Figure S3. UV-Vis spectra of the reaction mixture [PTH1 (10 mol\%) and LiBF (10 mol\%)]. 


\section{- Color Comparison of Solutions n}

The color of solutions containing reaction components was compared (Figure S4). The concentration of each components was identical to the catalytic reaction conditions $[\mathbf{3 a}(0.2 \mathrm{mmol})$, PTH1 $(1 \mathrm{~mol} \%)$ and $\mathrm{LiBF}_{4}(1 \mathrm{~mol} \%)$ in acetonitrile $(600 \mu \mathrm{l})$ solution]. A yellow-colored solution was obtained in the coexistence of PTH1 and redox active ester $\mathbf{3 a}$.

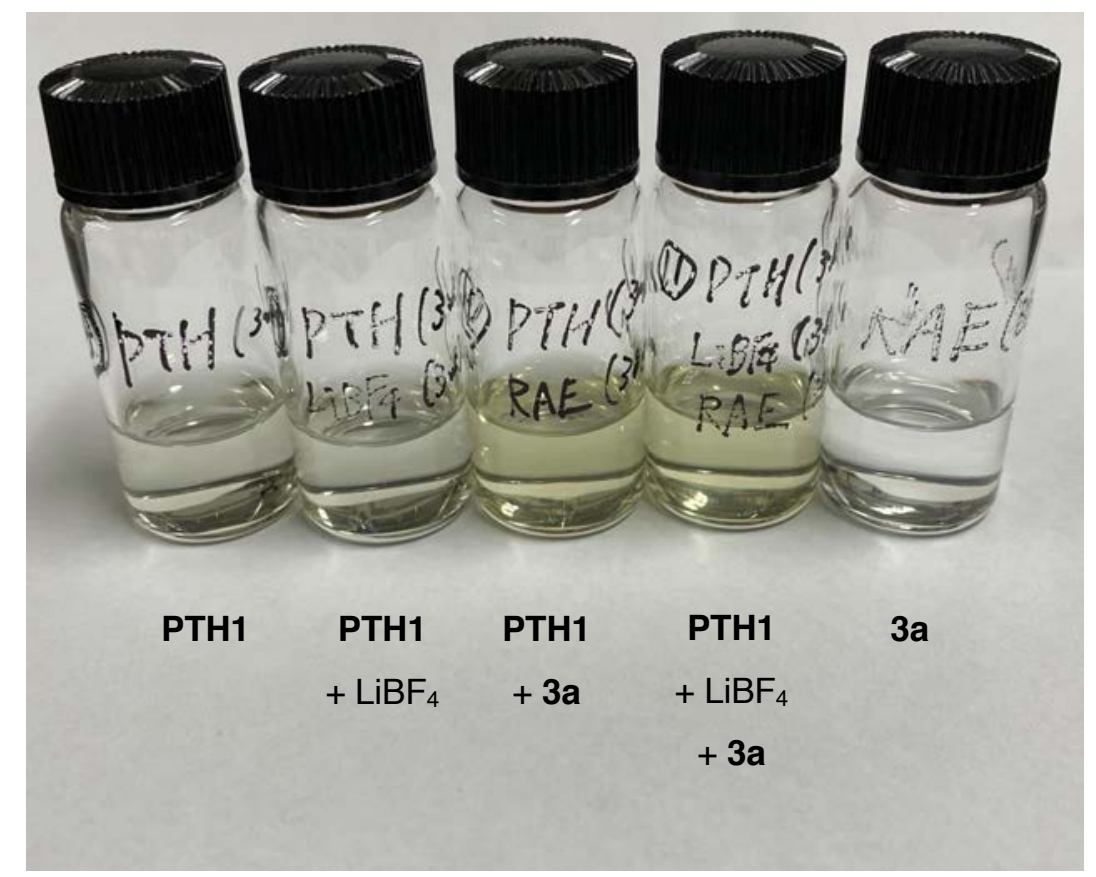

Figure S4. Color of Solutions

\section{- Stoichiometric Reaction}
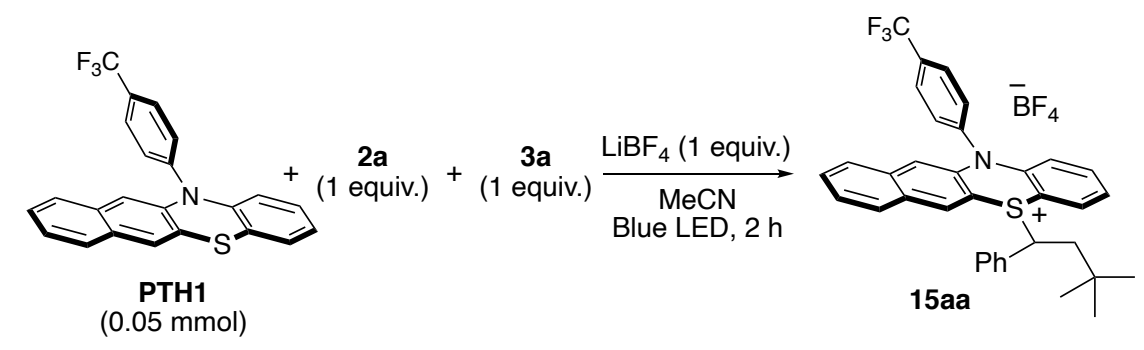

Figure S5. Stoichiometric Reaction

Figure S5 (Scheme 4B in manuscript). In a glovebox, to an oven-dried vial with a stirring bar was added redox active ester $\mathbf{3 a}(12.4 \mathrm{mg}, 0.05 \mathrm{mmol})$, styrene $\mathbf{2 a}(5.7 \mu 1,0.05 \mathrm{mmol})$, PTH1 (19.7 mg, $0.05 \mathrm{mmol}), \mathrm{LiBF}_{4}(4.7 \mathrm{mg}, 0.05 \mathrm{mmol})$ and $\mathrm{MeCN}(375 \mu \mathrm{l})$. After sealing the vial with a cap and removed from the glove box, the reaction was stirred and irradiated with a $34 \mathrm{~W}$ blue LED $(0.5 \mathrm{~cm}$ away) with a cooling fan to keep the temperature around $40{ }^{\circ} \mathrm{C}$ (Figure $\mathrm{S} 1$ ). After $2 \mathrm{~h}$, the reaction solution was directly used for MS analysis (HRMS-DART). 15aa: HRMS-DART $(\mathrm{m} / z)$ : $\left[\mathrm{M}-\mathrm{BF}_{4}\right]^{+}$ calcd for $\mathrm{C}_{35} \mathrm{H}_{31} \mathrm{~F}_{3} \mathrm{NS}$, 554.2129; found, 554.2115. 


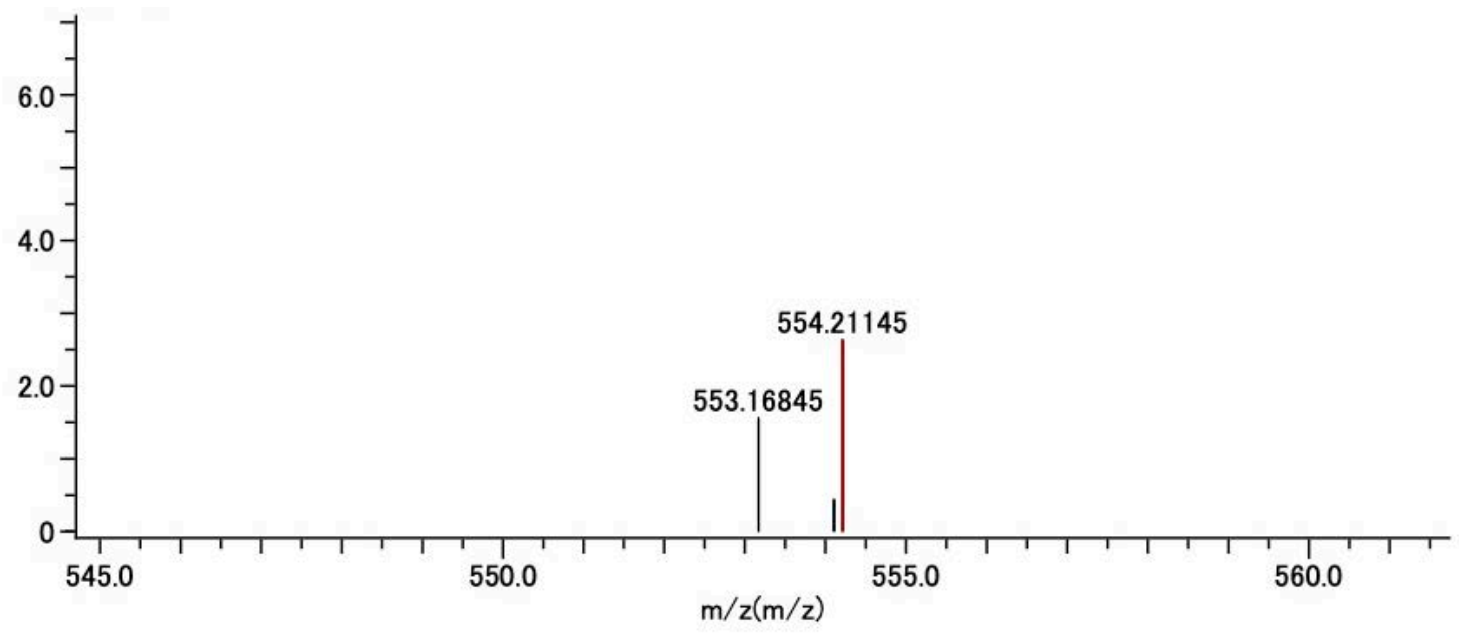

Figure S6. HRMS-DART spectrum of the reaction crude 


\section{- Cyclic Voltammetry of Organophotocatalysts}

$\mathrm{CV}$ measurements were carried out under nitrogen atmosphere in acetonitrile solutions with $0.1 \mathrm{M}$ of tetrabutylammonium tetrafluoroborate $\left({ }^{n} \mathrm{Bu}_{4} \mathrm{NBF}_{4}\right)$ as a supporting electrolyte. Measurements were made with a glassy carbon electrode $\left(\operatorname{area}=0.07 \mathrm{~cm}^{2}\right)$, an $\mathrm{Ag} / \mathrm{AgCl}$ reference electrode, and a Pt wire counter electrode. The concentration of the sample solution was fixed at $10 \mathrm{mM}$ and the sweep rates were set to $100 \mathrm{mV} / \mathrm{s}$.

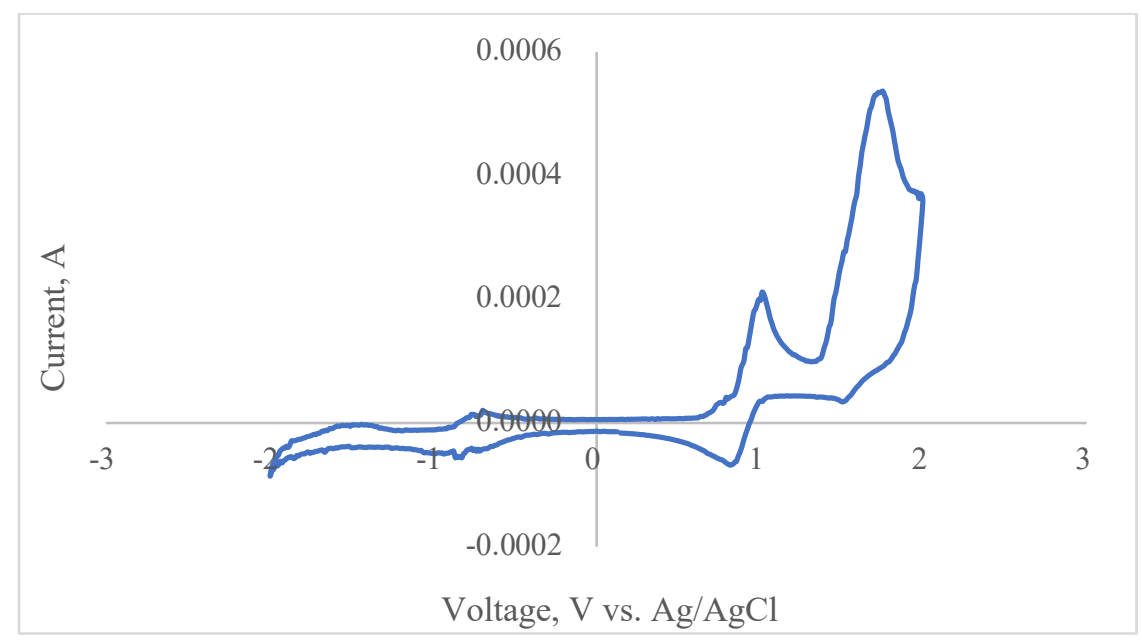

Figure S7. Cyclic voltammogram of PTH1.

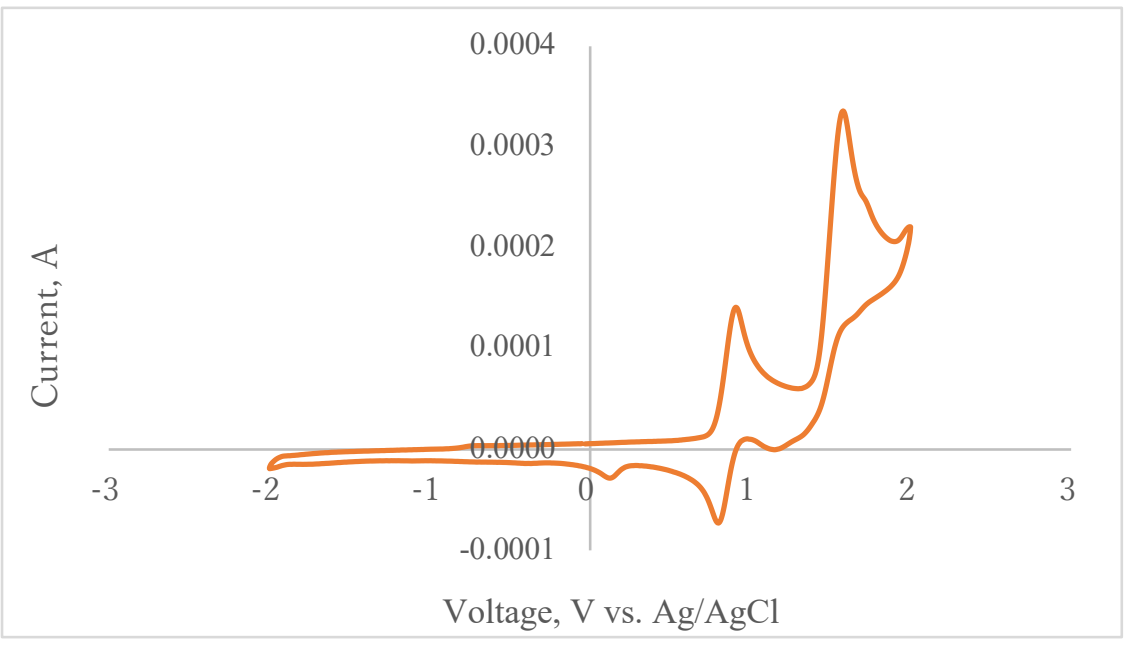

Figure S8. Cyclic voltammogram of PTH3.

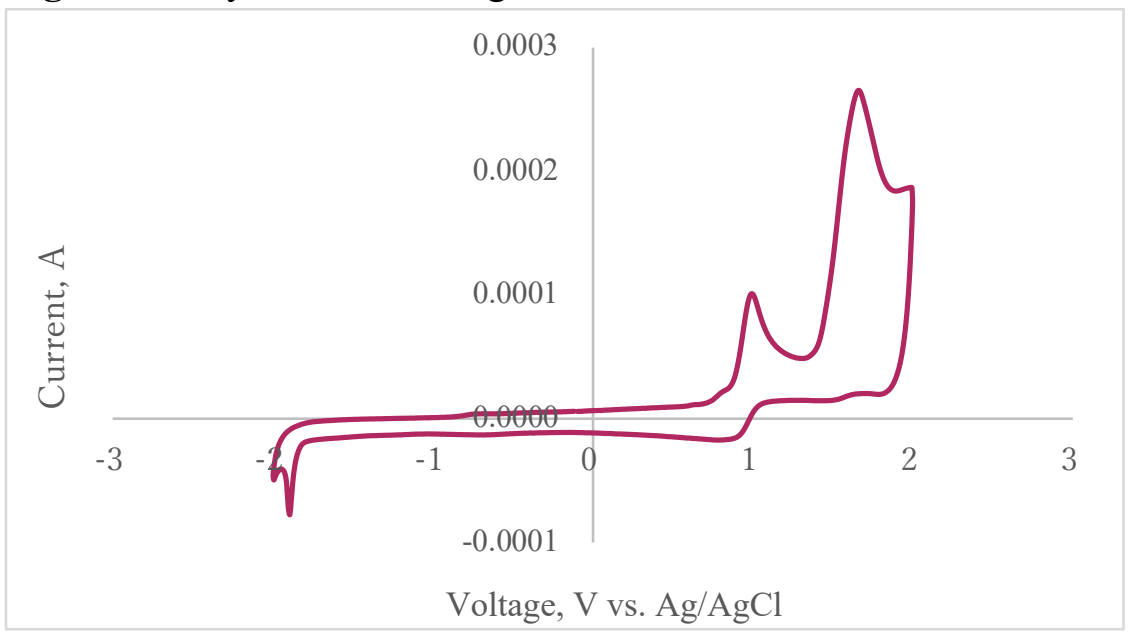

Figure S9. Cyclic voltammogram of PTH4 
- UV-Vis Spectra of Organophotocatalysts

UV-Vis adsorption spectra of organophotocatalysts were measured with $40 \mu \mathrm{M}$ acetonitrile solution.

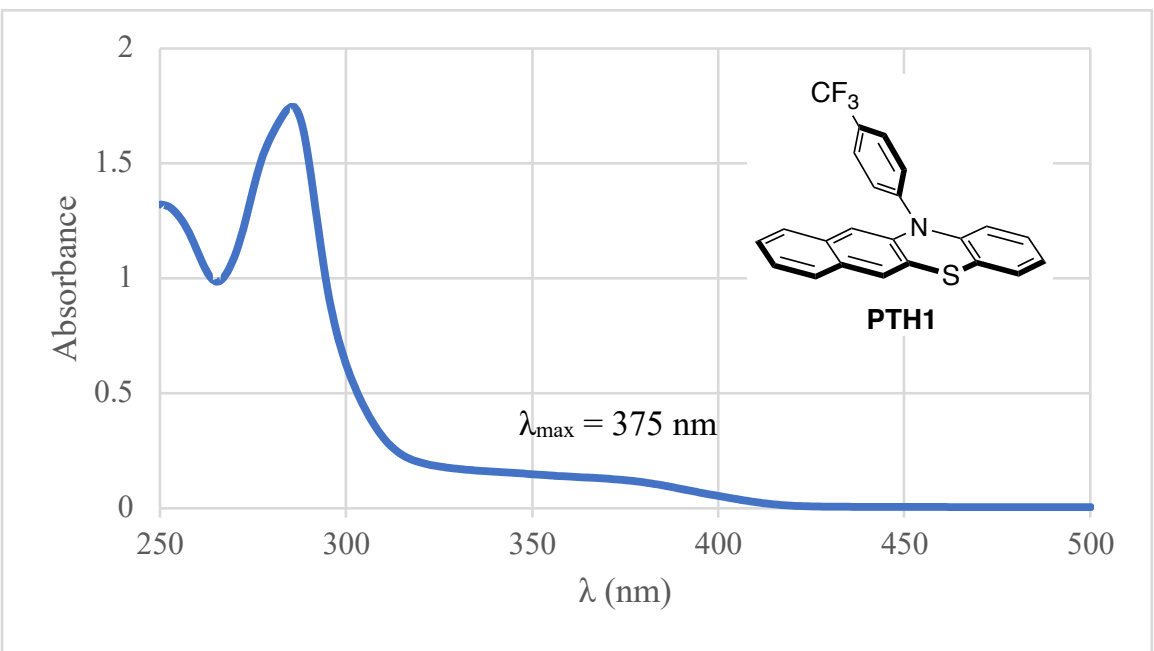

Figure S10. UV-vis spectrum of PTH1.

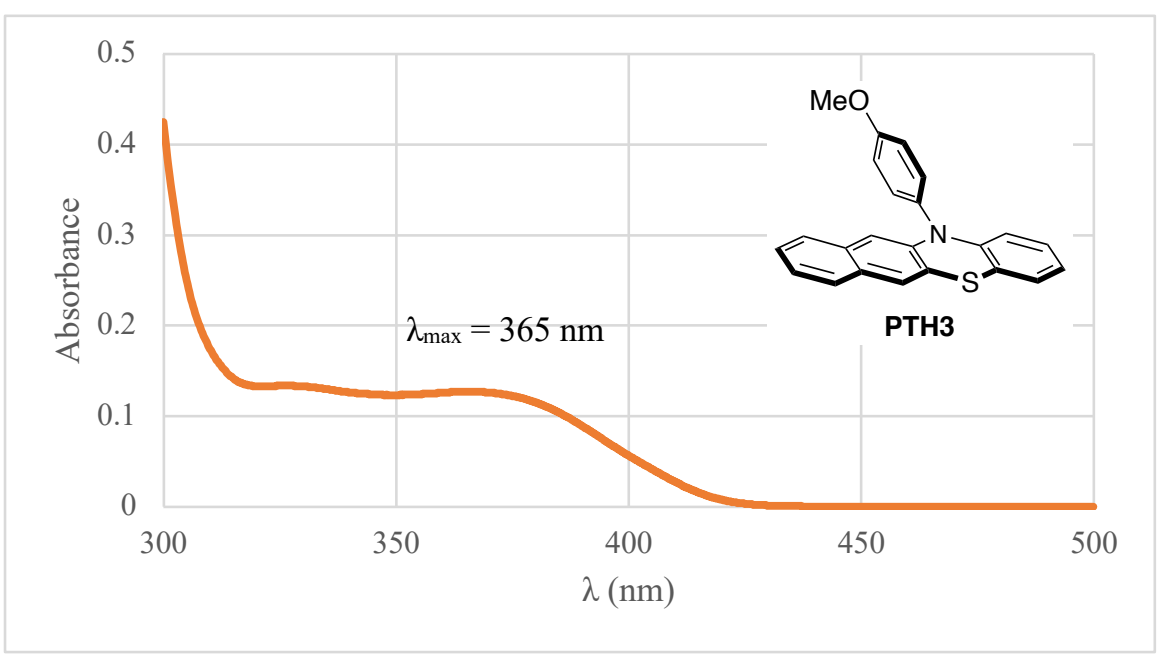

Figure S11. UV-Vis spectrum of PTH3.

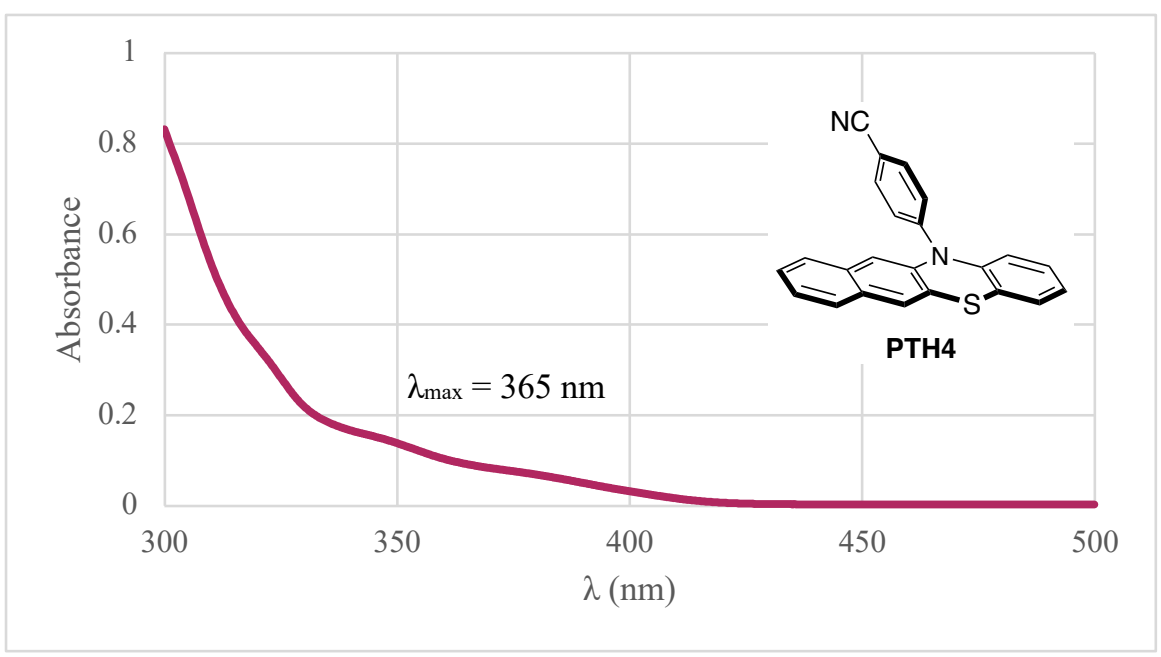

Figure S12. UV-Vis spectrum of PTH4. 
- Fluorescence Spectra of Organophotocatalysts

Fluorescence spectra of organophotocatalysts were measured with $40 \mu \mathrm{M}$ acetonitrile solution.
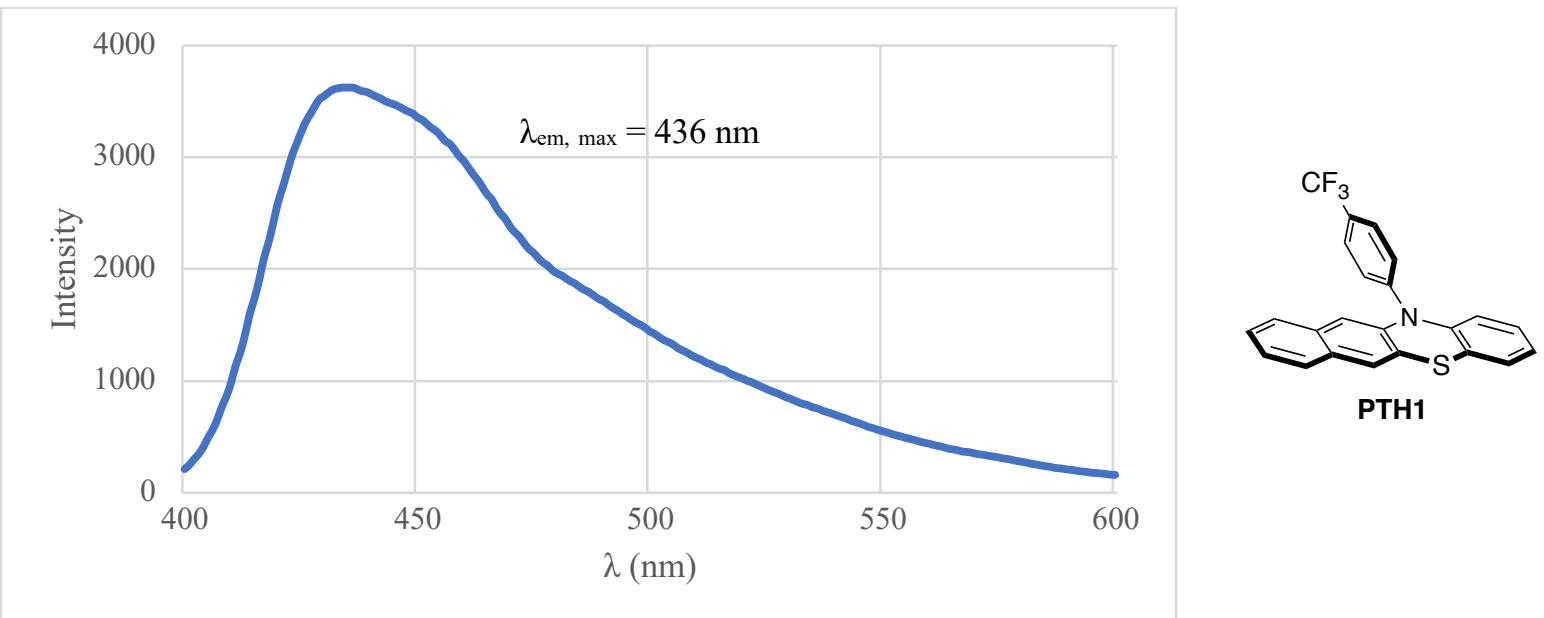

Figure S13. Fluorescence spectrum of PTH1 was scanned from 385 to $760 \mathrm{~nm}$ after excitation at 375 nm.
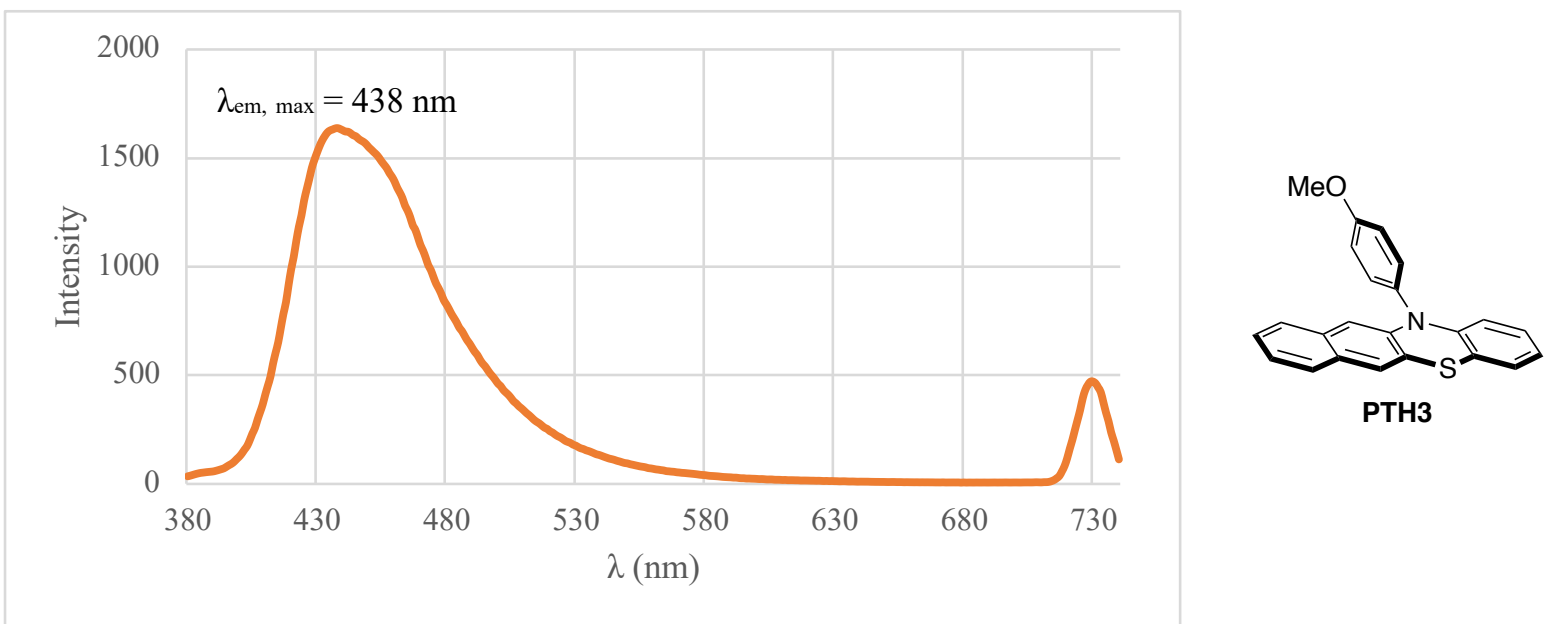

Figure S14. Fluorescence spectrum of PTH3 was scanned from 375 to $740 \mathrm{~nm}$ after excitation at 365 nm.
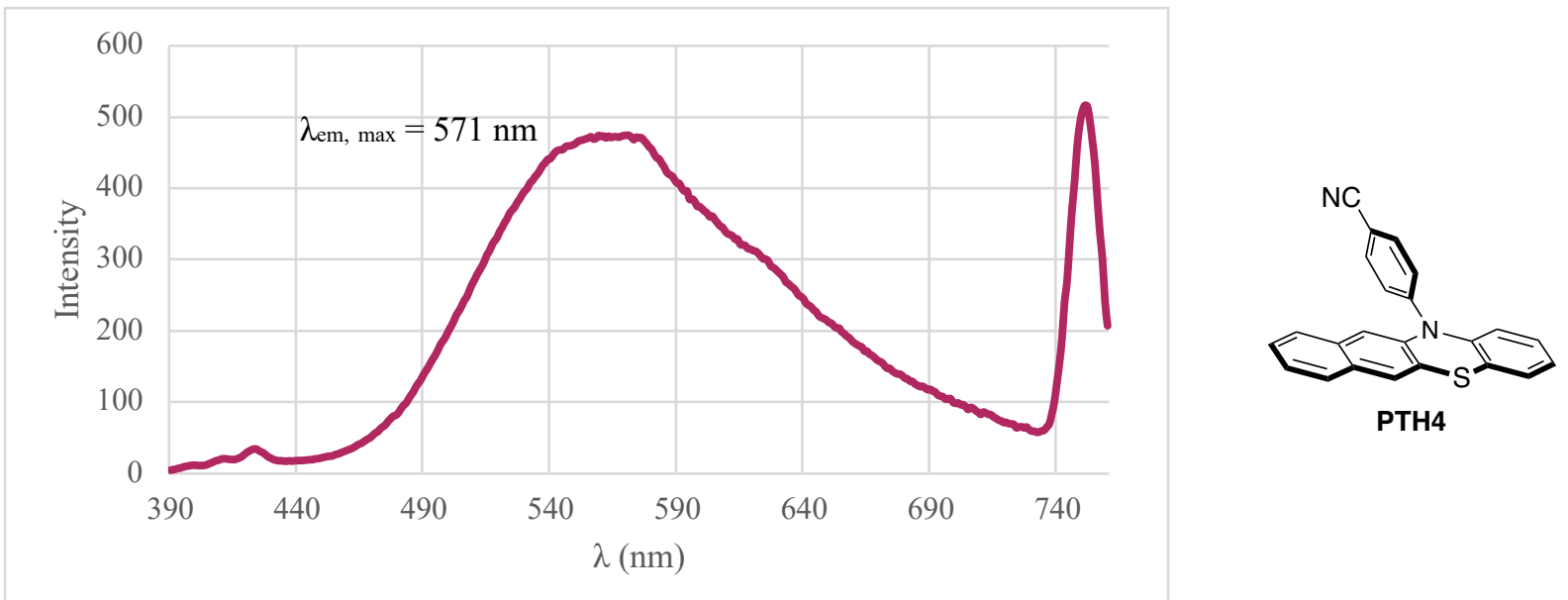

Figure S15. Fluorescence spectrum of PTH4 was scanned from 386 to $762 \mathrm{~nm}$ after excitation at 376 nm. 
- Summary of Redox Properties of Organophotocatalysts

\begin{tabular}{|c|c|c|c|c|}
\hline & PTH1 & PTH2 $^{a}$ & PTH3 & PTH4 \\
\hline $\mathrm{E}_{1 / 2}\left({ }^{2} \mathrm{PC}^{++} / 1 \mathrm{PC}\right)(\mathrm{V} \text { vs } \mathrm{Ag} / \mathrm{AgCl})^{b}$ & 0.91 & - & 0.86 & 0.89 \\
\hline $\mathrm{E}_{1 / 2}\left({ }^{2} \mathrm{PC}{ }^{\circ+} / 1 \mathrm{PC}\right)(\mathrm{V} \text { vs SCE })^{c}$ & 0.87 & 0.85 & 0.81 & 0.84 \\
\hline$\lambda_{\max , \mathrm{em}}(\mathrm{nm})$ & 436 & 439 & 438 & 571 \\
\hline $\mathrm{E}_{\mathrm{S} 1, \exp }(\mathrm{eV})^{d}$ & 2.84 & 2.82 & 2.83 & 2.17 \\
\hline $\mathrm{E}^{0^{*}} \mathrm{~S} 1, \exp \left({ }^{2} \mathrm{PC}^{\cdot+} / 1 \mathrm{PC}^{*}\right)(\mathrm{V} \text { vs SCE})^{e}$ & -1.97 & -1.97 & -2.02 & -1.33 \\
\hline
\end{tabular}

${ }^{a}$ ref $16 .{ }^{b}$ Determined by CV experiments. ${ }^{c} \mathrm{E}_{1 / 2}(\mathrm{Ag} / \mathrm{AgCl})(\mathrm{V}$ vs SCE$)=0.045 \mathrm{~V} .{ }^{d}$ Singlet energies were calculated using the maximum wavelength of emission. ${ }^{e}$ Singlet excited state reduction potentials were calculated using the singlet energies and the $\mathrm{E}_{1 / 2}$.

\section{Computational Study on Gibbs Energies of Benzyl Cation and Benzyl Sulfonium}

All calculations were carried out with Gaussian 09, Revision D.01. ${ }^{17}$ The density functional theory (DFT) functional B3LYP was used with the 6-31G(d,p) basis set for geometry optimizations. We carried out vibrational frequency calculations with $6-31 \mathrm{G}(\mathrm{d}, \mathrm{p})$ to obtain thermal corrections to Gibbs free energies $(\mathrm{G})$ at $298.15 \mathrm{~K}\left(25^{\circ} \mathrm{C}\right)$.

\begin{tabular}{|c|c|c|c|}
\hline & & \\
& -1637.129968 & -309.896079 & -1947.049654 \\
\hline Gibbs Free energy (hartree) & -1637.129968 & -309.896079 & -1947.049654 \\
\hline Gibbs Free energy (hartree) & -1027315.426 & -194462.8885 & -1221793.128 \\
\hline Gibbs Free energy (kcal/mol) & -1221778.315 & -1221793.128 \\
\hline total (kcal/mol) & \multicolumn{2}{|c|}{0} \\
\hline$\Delta \mathrm{G}(\mathrm{kcal} / \mathrm{mol})$ & & -14.81362857 \\
\hline
\end{tabular}




\section{(1) PTH1}

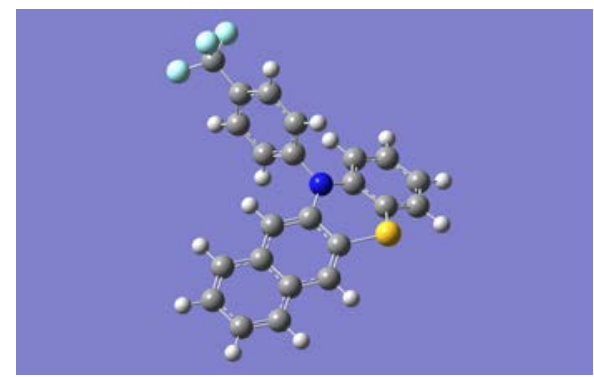

\begin{tabular}{|c|c|c|c|}
\hline $\mathrm{C}$ & -3.23031700 & -4.48003700 & 0.58430000 \\
\hline $\mathrm{C}$ & -2.15070900 & -3.62431200 & 0.55974600 \\
\hline $\mathrm{C}$ & -2.31991000 & -2.25041700 & 0.24298900 \\
\hline $\mathrm{C}$ & -3.63355700 & -1.77140600 & -0.05324200 \\
\hline $\mathrm{C}$ & -4.72614300 & -2.67563800 & -0.02139100 \\
\hline $\mathrm{C}$ & -4.53036500 & -4.00297500 & 0.29170600 \\
\hline $\mathrm{C}$ & -1.22909800 & -1.34197400 & 0.23099500 \\
\hline $\mathrm{C}$ & -3.78602300 & -0.39859300 & -0.37669300 \\
\hline $\mathrm{C}$ & -2.70693100 & 0.45387100 & -0.42394200 \\
\hline $\mathrm{C}$ & -1.39350700 & -0.00581500 & -0.08967600 \\
\hline $\mathrm{C}$ & -0.46246200 & 2.28270200 & 0.17657500 \\
\hline $\mathrm{C}$ & -1.66429000 & 2.94440400 & -0.14256800 \\
\hline $\mathrm{H}$ & -0.24632600 & -1.72605300 & 0.47502100 \\
\hline $\mathrm{H}$ & -3.08584500 & -5.52824300 & 0.82903800 \\
\hline $\mathrm{H}$ & -1.15281000 & -3.99179700 & 0.78446600 \\
\hline $\mathrm{H}$ & -5.72157600 & -2.30322100 & -0.24885500 \\
\hline $\mathrm{H}$ & -5.37283300 & -4.68765100 & 0.31464100 \\
\hline $\mathrm{H}$ & -4.77482200 & -0.01841900 & -0.61789700 \\
\hline $\mathrm{C}$ & 0.56481200 & 3.03210000 & 0.77201100 \\
\hline $\mathrm{C}$ & 0.39818600 & 4.39044500 & 1.04000200 \\
\hline $\mathrm{H}$ & 1.21647800 & 4.94259700 & 1.49203300 \\
\hline $\mathrm{C}$ & -0.80429600 & 5.02919100 & 0.74660300 \\
\hline $\mathrm{H}$ & -0.94170700 & 6.08304100 & 0.96572900 \\
\hline $\mathrm{C}$ & -1.83637100 & 4.29527000 & 0.16393800 \\
\hline $\mathrm{N}$ & -0.30204700 & 0.90210300 & -0.11951400 \\
\hline $\mathrm{S}$ & -2.93522500 & 2.09962800 & -1.05458700 \\
\hline $\mathrm{H}$ & 1.50521700 & 2.55601000 & 1.01815700 \\
\hline $\mathrm{H}$ & -2.78256100 & 4.77087100 & -0.07610000 \\
\hline $\mathrm{C}$ & 1.02757900 & 0.35704600 & -0.10299900 \\
\hline $\mathrm{C}$ & 1.71565500 & 0.22444300 & -1.31162200 \\
\hline
\end{tabular}




$\begin{array}{lrrr}\mathrm{C} & 1.63780100 & -0.04320300 & 1.09247500 \\ \mathrm{C} & 3.00640000 & -0.30061700 & -1.32906600 \\ \mathrm{H} & 1.22907900 & 0.53308500 & -2.23064600 \\ \mathrm{C} & 2.92782600 & -0.56854200 & 1.07684700 \\ \mathrm{H} & 1.09635000 & 0.05692700 & 2.02754500 \\ \mathrm{C} & 3.61240200 & -0.69685700 & -0.13482200 \\ \mathrm{H} & 3.53890400 & -0.41014600 & -2.26718700 \\ \mathrm{H} & 3.39986600 & -0.88571400 & 2.00021900 \\ \mathrm{C} & 5.02798600 & -1.21030100 & -0.14236800 \\ \mathrm{~F} & 5.33889900 & -1.81113100 & -1.31133100 \\ \mathrm{~F} & 5.24097000 & -2.10608100 & 0.84687400 \\ \mathrm{~F} & 5.92041100 & -0.20865100 & 0.03612700\end{array}$

\section{(2) Benzyl cation}

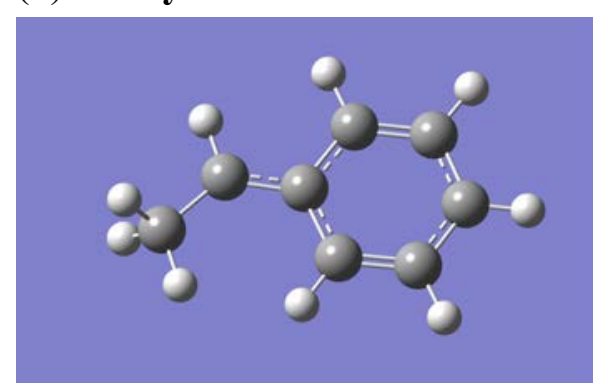

$\begin{array}{lrrr}\mathrm{C} & -2.28592400 & -0.30323900 & -0.00000300 \\ \mathrm{C} & -1.86029200 & 1.03603300 & -0.00000100 \\ \mathrm{C} & -0.50895400 & 1.31333600 & 0.00000300 \\ \mathrm{C} & 0.45219100 & 0.24615300 & 0.00000400 \\ \mathrm{C} & -0.01467700 & -1.11080200 & 0.00000400 \\ \mathrm{C} & -1.36770800 & -1.37192600 & -0.00000100 \\ \mathrm{H} & -3.35011700 & -0.52130200 & -0.00000400 \\ \mathrm{H} & -2.58921300 & 1.83863600 & -0.00000300 \\ \mathrm{H} & -0.15634700 & 2.34050900 & 0.00000500 \\ \mathrm{H} & 0.69551100 & -1.92939500 & 0.00000800 \\ \mathrm{H} & -1.72998200 & -2.39393200 & -0.00000200 \\ \mathrm{C} & 1.80217200 & 0.58059600 & -0.00000100 \\ \mathrm{H} & 2.03079300 & 1.64738800 & -0.00000300 \\ \mathrm{C} & 2.97331700 & -0.30599200 & -0.00000300 \\ \mathrm{H} & 3.60207400 & -0.05734200 & -0.86870700 \\ \mathrm{H} & 3.60208300 & -0.05733800 & 0.86869400 \\ \mathrm{H} & 2.75444800 & -1.37217500 & 0.00000000\end{array}$




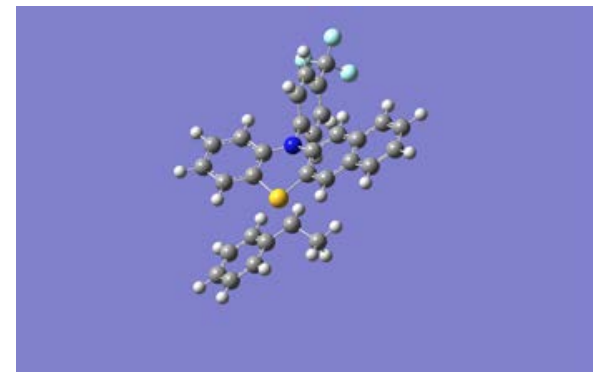

\begin{tabular}{|c|c|c|c|}
\hline $\mathrm{C}$ & 0.70014147 & -0.72842998 & 0.00000000 \\
\hline $\mathrm{C}$ & 0.38347747 & -2.06706298 & 0.01108200 \\
\hline $\mathrm{C}$ & 1.39017647 & -3.04336298 & 0.24382800 \\
\hline $\mathrm{C}$ & 2.73584147 & -2.59992898 & 0.46299200 \\
\hline $\mathrm{C}$ & 3.03160247 & -1.20950298 & 0.44731500 \\
\hline $\mathrm{C}$ & 2.03283547 & -0.29337398 & 0.22033800 \\
\hline $\mathrm{C}$ & 1.08805347 & -4.42544698 & 0.28474000 \\
\hline $\mathrm{C}$ & 3.72383447 & -3.58247298 & 0.67454400 \\
\hline $\mathrm{C}$ & 3.40181547 & -4.92870198 & 0.67682700 \\
\hline $\mathrm{C}$ & 2.05854947 & -5.39157298 & 0.51538700 \\
\hline $\mathrm{C}$ & 2.57470747 & -7.72877798 & 1.16637400 \\
\hline $\mathrm{C}$ & 3.95626147 & -7.51279598 & 1.37842200 \\
\hline $\mathrm{H}$ & 0.05701947 & -4.72389398 & 0.14693200 \\
\hline $\mathrm{H}$ & -0.07684453 & 0.00817302 & -0.17819300 \\
\hline $\mathrm{H}$ & -0.63895053 & -2.39268898 & -0.15522900 \\
\hline $\mathrm{H}$ & 4.05404147 & -0.88455898 & 0.61686300 \\
\hline $\mathrm{H}$ & 2.25825747 & 0.76785302 & 0.20921000 \\
\hline $\mathrm{H}$ & 4.75475147 & -3.27461198 & 0.82867400 \\
\hline $\mathrm{C}$ & 2.07006447 & -8.98397398 & 1.56856900 \\
\hline $\mathrm{C}$ & 2.88698947 & -9.93019498 & 2.17374100 \\
\hline $\mathrm{H}$ & 2.45119947 & -10.87798198 & 2.47321900 \\
\hline $\mathrm{C}$ & 4.24013447 & -9.67639698 & 2.41927000 \\
\hline $\mathrm{H}$ & 4.86541947 & -10.41219298 & 2.91151300 \\
\hline $\mathrm{C}$ & 4.77163447 & -8.46104898 & 2.01622700 \\
\hline $\mathrm{N}$ & 1.72843147 & -6.76161798 & 0.60383300 \\
\hline $\mathrm{S}$ & 4.76333347 & -6.05092998 & 0.80270100 \\
\hline $\mathrm{H}$ & 1.02340547 & -9.20894298 & 1.41667000 \\
\hline $\mathrm{H}$ & 5.81973347 & -8.23441998 & 2.18131300 \\
\hline $\mathrm{C}$ & 0.35630347 & -7.13617198 & 0.33226700 \\
\hline $\mathrm{C}$ & 0.00766747 & -7.57064998 & -0.94720600 \\
\hline
\end{tabular}




$\begin{array}{lllr}\mathrm{C} & -0.60766753 & -7.05512398 & 1.34217300 \\ \mathrm{C} & -1.31126053 & -7.93173098 & -1.21926500 \\ \mathrm{H} & 0.76362847 & -7.62270098 & -1.72360400 \\ \mathrm{C} & -1.92492953 & -7.41638998 & 1.06822600 \\ \mathrm{H} & -0.32550153 & -6.70980398 & 2.33156000 \\ \mathrm{C} & -2.27487753 & -7.85545298 & -0.21177200 \\ \mathrm{H} & -1.59286653 & -8.26420398 & -2.21188500 \\ \mathrm{H} & -2.68090853 & -7.35098298 & 1.84262600 \\ \mathrm{C} & -3.69198453 & -8.30093698 & -0.48921900 \\ \mathrm{~F} & -4.01670953 & -8.13125098 & -1.78598300 \\ \mathrm{~F} & -4.57789553 & -7.61168198 & 0.25665000 \\ \mathrm{~F} & -3.85083253 & -9.60914498 & -0.19947400 \\ \mathrm{C} & 5.33862447 & -6.41118198 & -1.03574100 \\ \mathrm{H} & 5.61694447 & -5.38742898 & -1.30721200 \\ \mathrm{C} & 4.19258847 & -6.90278198 & -1.90295000 \\ \mathrm{H} & 3.38539047 & -6.16868098 & -1.94616700 \\ \mathrm{H} & -9.79982847 & -9.42131398 & -0.68105700 \\ \mathrm{H} & & & -2.91942200 \\ \mathrm{C} & & & \\ \mathrm{H} & & & \end{array}$




\section{- References匹}

(1) Odell, L. R.; Lindh, J.; Gustafsson, T.; Larhed, M. Eur. J. Org. Chem. 2010, 2270-2274.

(2) Sakaguchi, H.; Uetake, Y.; Ohashi, M.; Niwa, T.; Ogoshi, S.; Hosoya, T. J. Am. Chem. Soc. 2017, 139, 12855-12862.

(3) Kondoh, A.; Koda, K.; Terada, M. Org. Lett. 2019, 21, 2277-2280.

(4) Chan, C.-M.; Xing, Q.; Chow, Y.-C.; Hung, S.-F.; Yu, W.-Y. Org. Lett. 2019, 21, 8037-8043.

(5) Ghosh, K. K.; Uttry, A.; Koldemir, A.; Ong, M.; van Gemmeren, M. Org. Lett. 2019, 21, $7154-7157$.

(6) Correia, J. T. M.; Gustavo Piva da Silva, G. P. da.; Kisukuri, C. M.; André, E.; Pires, B.; Carneiro, P. S.; Paixão, M. W. J. Org. Chem. 2020, 85, 9820-9834.

(7) Qin, T.; Malins, L. R.; Edwards, J. T.; Merchant, R. R.; Novak, A. J. E.; Zhong, J. Z.; Mills, R. B.; Yan, M.; Yuan, C.; Eastgate, M. D.; Baran, P. S. Angew. Chem., Int. Ed. 2017, 56, 260-265.

(8) Li, H.; Breen, C. P.; Seo, H.; Jamison, T. F.; Fang, Y.-Q.; Bio, M. M. Org. Lett. 2018, 20, $1338-1341$.

(9) Qin, T.; Cornella, J.; Li, C.; Malins, L. R.; Edwards, J. T.; Kawamura, S.; Maxwell, B. D.; Eastgate, M. D.; Baran, P. S. Science 2016, 352, 801-805.

(10) Antonchick, A. P.; Samanta, R.; Kulikov, K.; Lategahn, J. Angew. Chem., Int. Ed. 2011, 50, $8605-8608$.

(11) Ghosh, T.; Jana, S.; Dash, J. Org. Lett. 2019, 21, 6690- 6694.

(12) M, Maria V.; H, Daniel A.; L, Enrique L.; B, Andrea B. J.; K, Teodoro S. RSC Adv. 2017. 45. 28298-28307.

(13) Dadashi-Silab, S.; Pan, X.; Matyjaszewski, K. Chem. Eur. J. 2017, 23, 5972-5977.

(14) Kennedy, N.; Lu, G.; Liu, P.; Cohen, T. J. Org. Chem. 2015, 80, 8571-8582.

(15) Shimizu, N.; Watanabe, S.; Tsuno, Y. Bull. Chem. Soc. Jpn. 1991, 64, 2249-2254.

(16) Shibutani, S.; Kodo, T.; Takeda, M.; Nagao, K.; Tokunaga, N.; Sasaki, Y.; Ohmiya, H. J. Am. Chem. Soc. 2020, 142, 1211-1216.

(17) Gaussian 09, Revision D.01, M. J. Frisch, G. W. Trucks, H. B. Schlegel, G. E. Scuseria, M. A. Robb, J. R. Cheeseman, G. Scalmani, V. Barone, B. Mennucci, G. A. Petersson, H. Nakatsuji, M. Caricato, X. Li, H. P. Hratchian, A. F. Izmaylov, J. Bloino, G. Zheng, J. L. Sonnenberg, M. Hada, M. Ehara, K. Toyota, R. Fukuda, J. Hasegawa, M. Ishida, T. Nakajima, Y. Honda, O. Kitao, H. Nakai, T. Vreven, J. A. Montgomery, Jr., J. E. Peralta, F. Ogliaro, M. Bearpark, J. J. Heyd, E. Brothers, K. N. Kudin, V. N. Staroverov, T. Keith, R. Kobayashi, J. Normand, K. Raghavachari, A. Rendell, J. C. Burant, S. S. Iyengar, J. Tomasi, M. Cossi, N. Rega, J. M. Millam, M. Klene, J. E. Knox, J. B. Cross, V. Bakken, C. Adamo, J. Jaramillo, R. Gomperts, R. E. Stratmann, O. Yazyev, A. J. Austin, R. Cammi, C. Pomelli, J. W. Ochterski, R. L. Martin, K. Morokuma, V. G. Zakrzewski, G. A. Voth, P. Salvador, J. J. Dannenberg, S. Dapprich, A. D. Daniels, O. Farkas, J. B. Foresman, J. V. Ortiz, J. Cioslowski, and D. J. Fox, Gaussian, Inc., Wallingford CT, 2013 


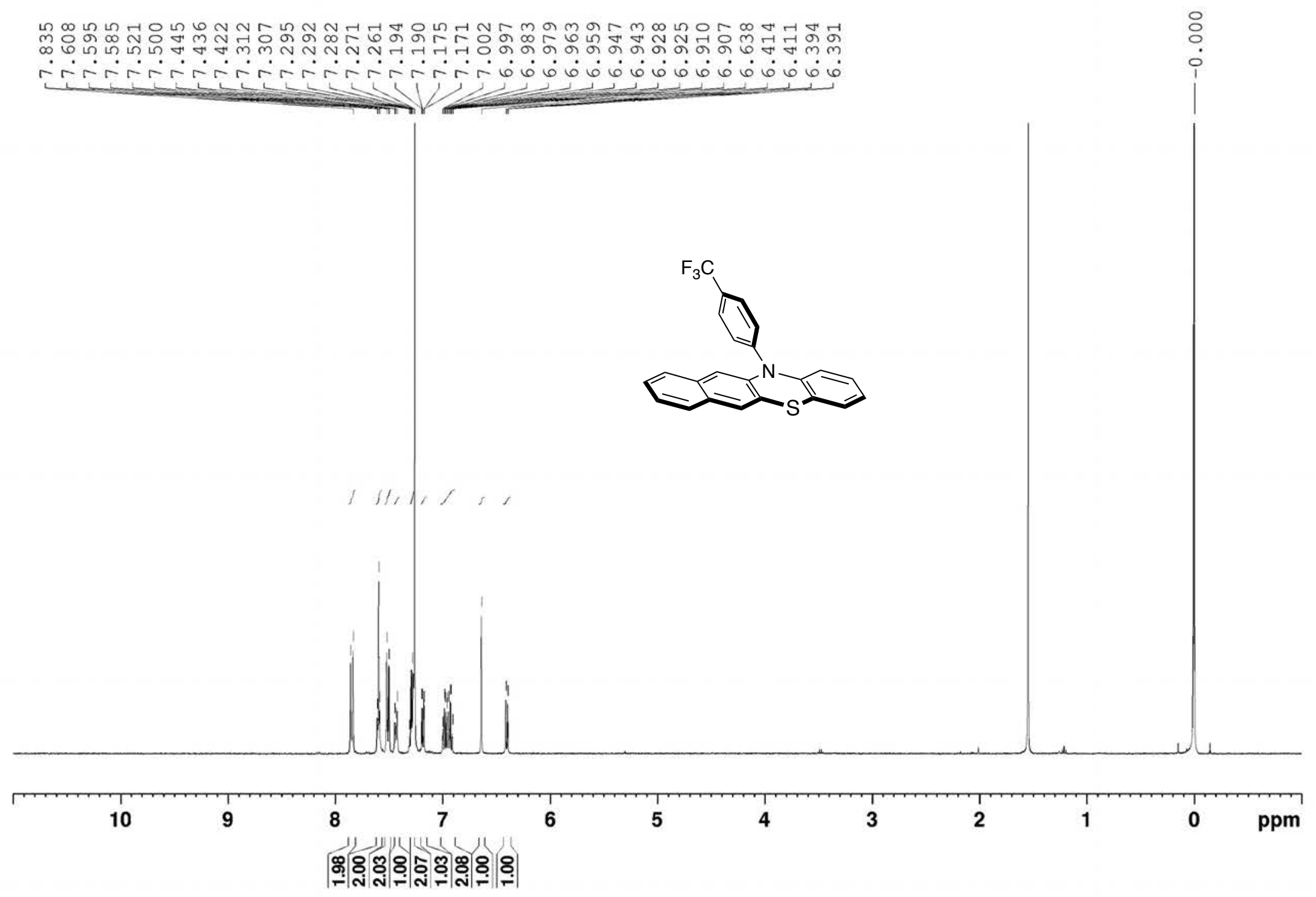

${ }^{1} \mathrm{H}$ NMR spectrum of PTH1 (400 MHz, $\mathrm{CDCl}_{3}$ ) 

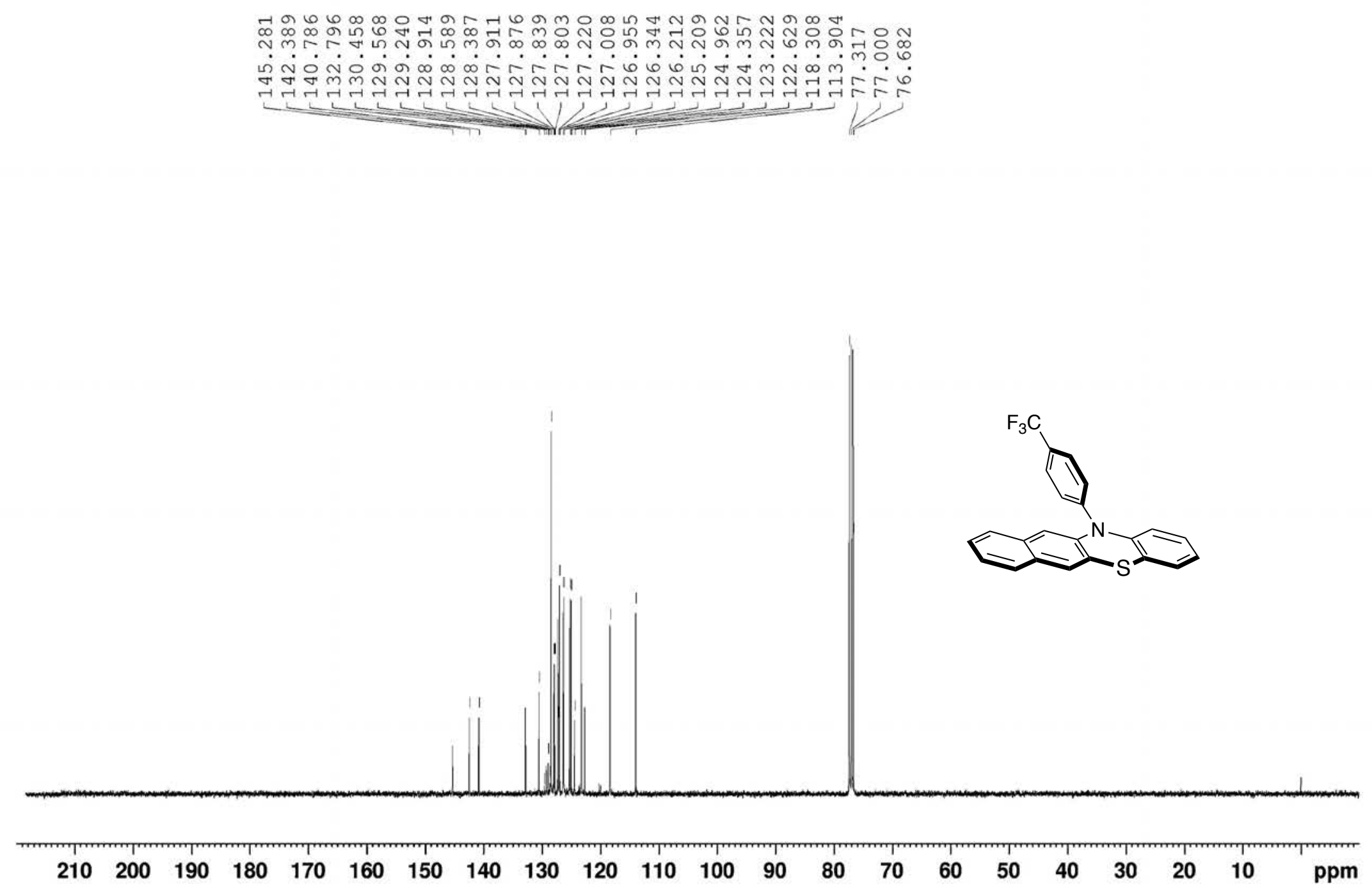

${ }^{13} \mathrm{C}$ NMR spectrum of PTH1 $\left(100.6 \mathrm{MHz}, \mathrm{CDCl}_{3}\right)$ 


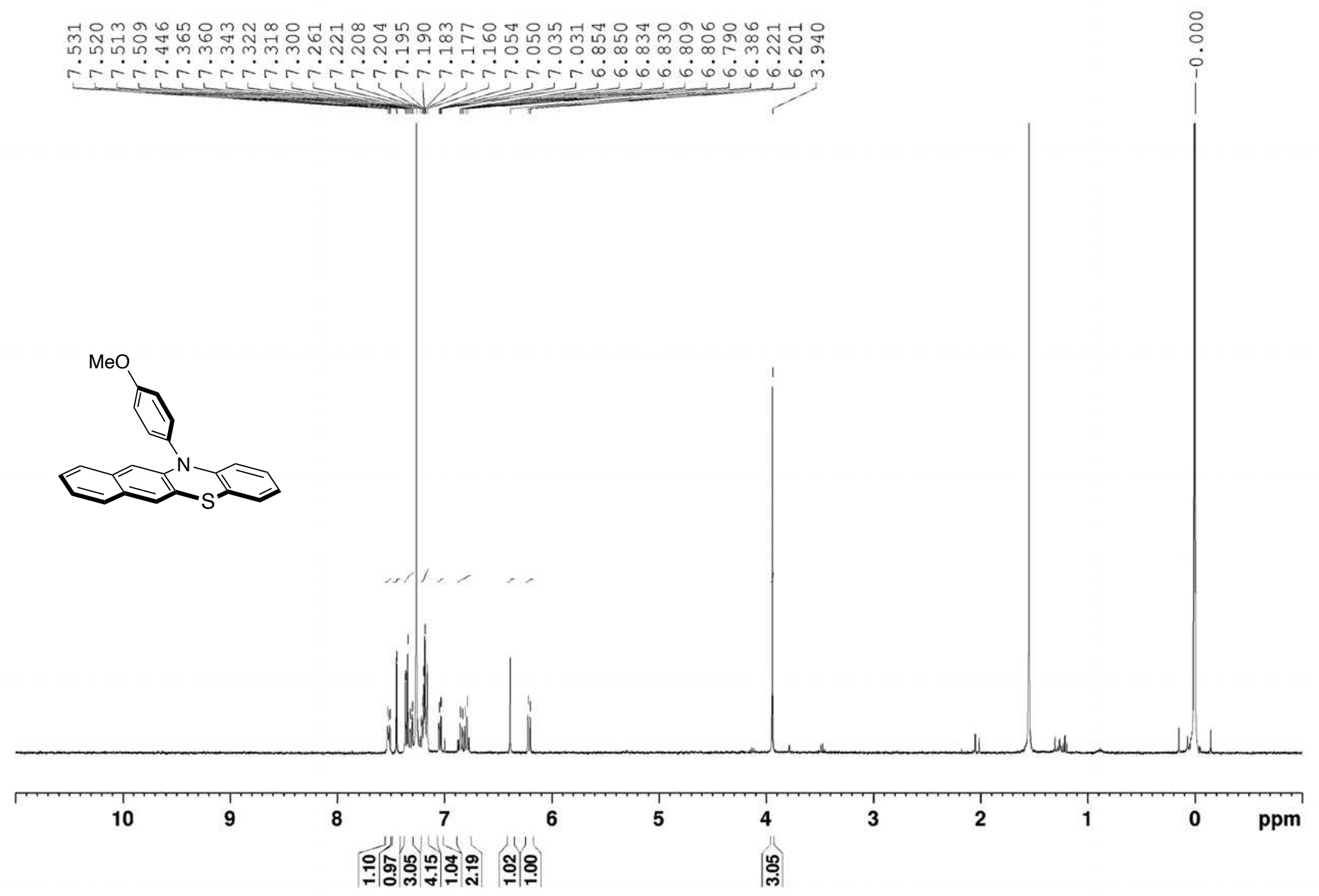

${ }^{1} \mathrm{H}$ NMR spectrum of PTH3 (400 MHz, $\mathrm{CDCl}_{3}$ ) 


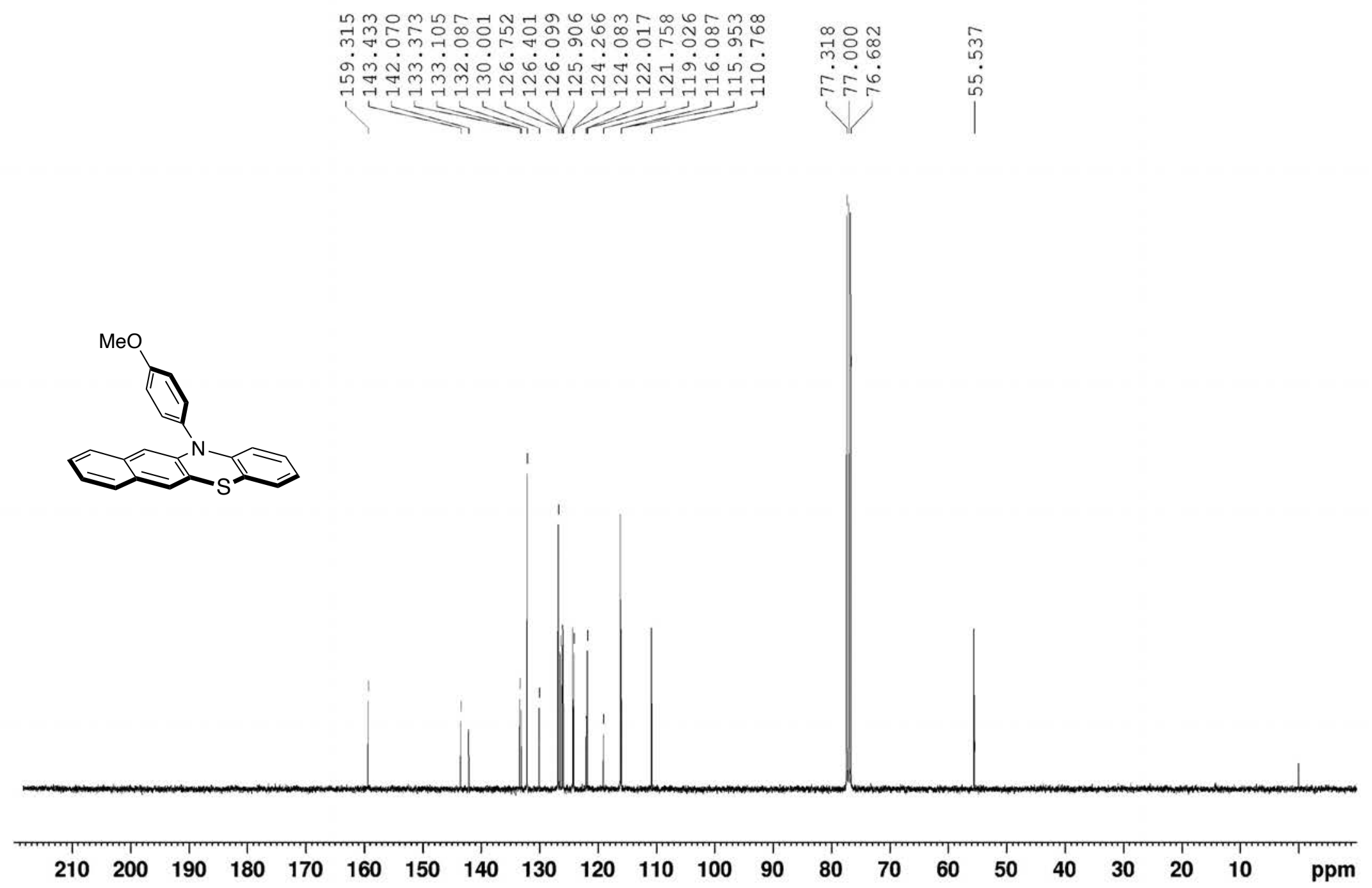

${ }^{13} \mathrm{C}$ NMR spectrum of PTH3 (100.6 MHz, $\left.\mathrm{CDCl}_{3}\right)$ 


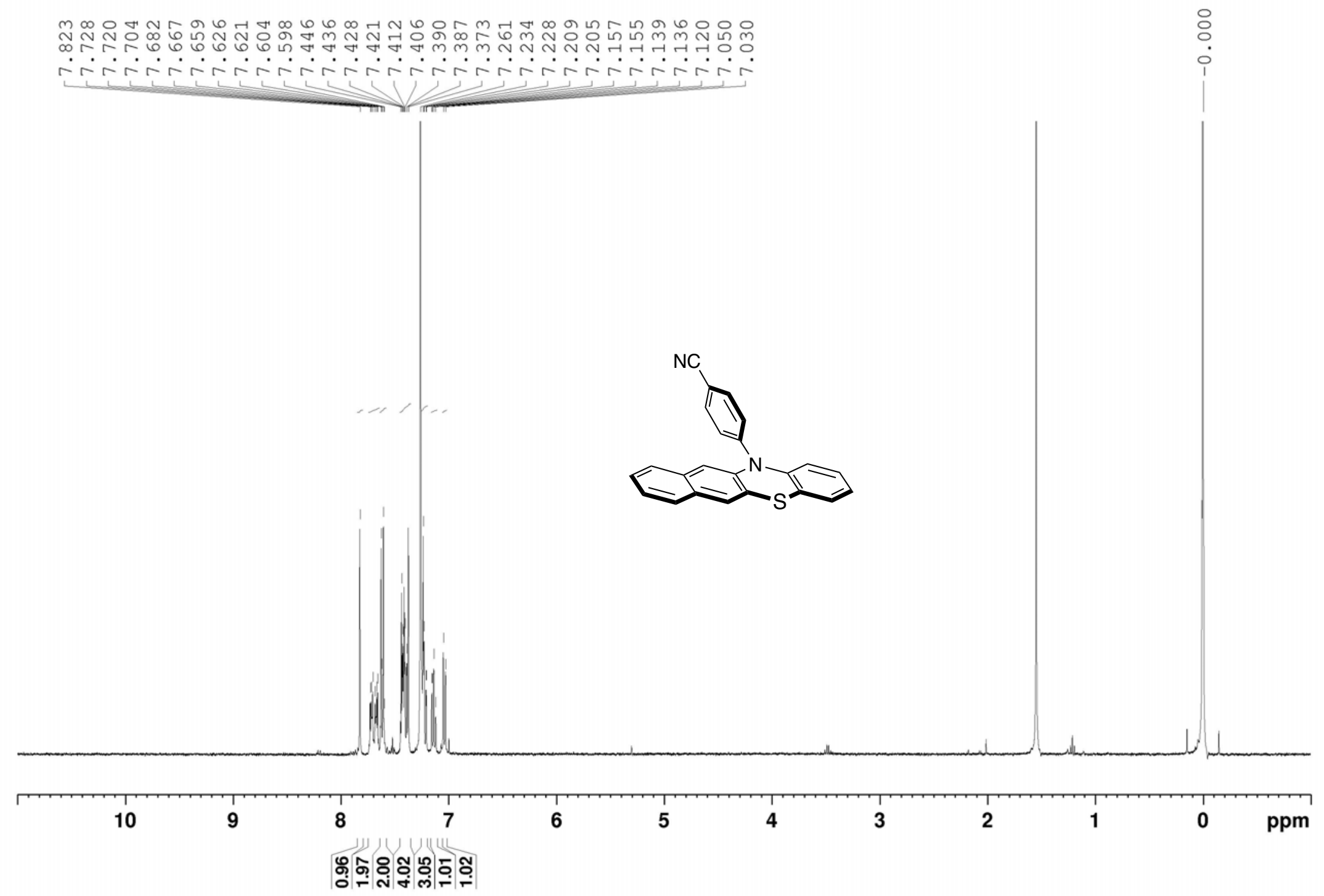

${ }^{1} \mathrm{H}$ NMR spectrum of PTH4 (400 MHz, $\mathrm{CDCl}_{3}$ ) 


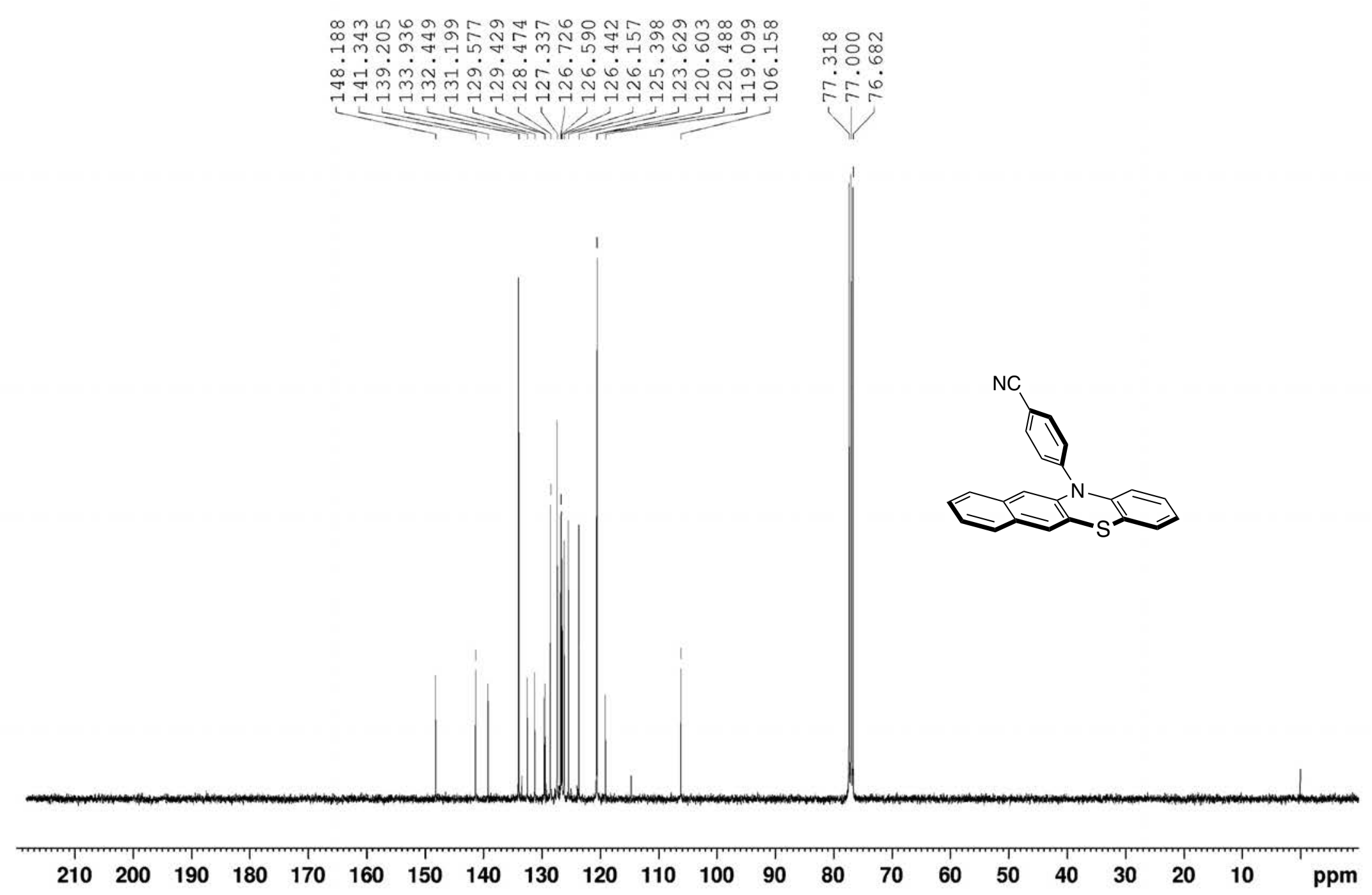

${ }^{13} \mathrm{C}$ NMR spectrum of PTH4 (100.6 MHz, $\left.\mathrm{CDCl}_{3}\right)$ 


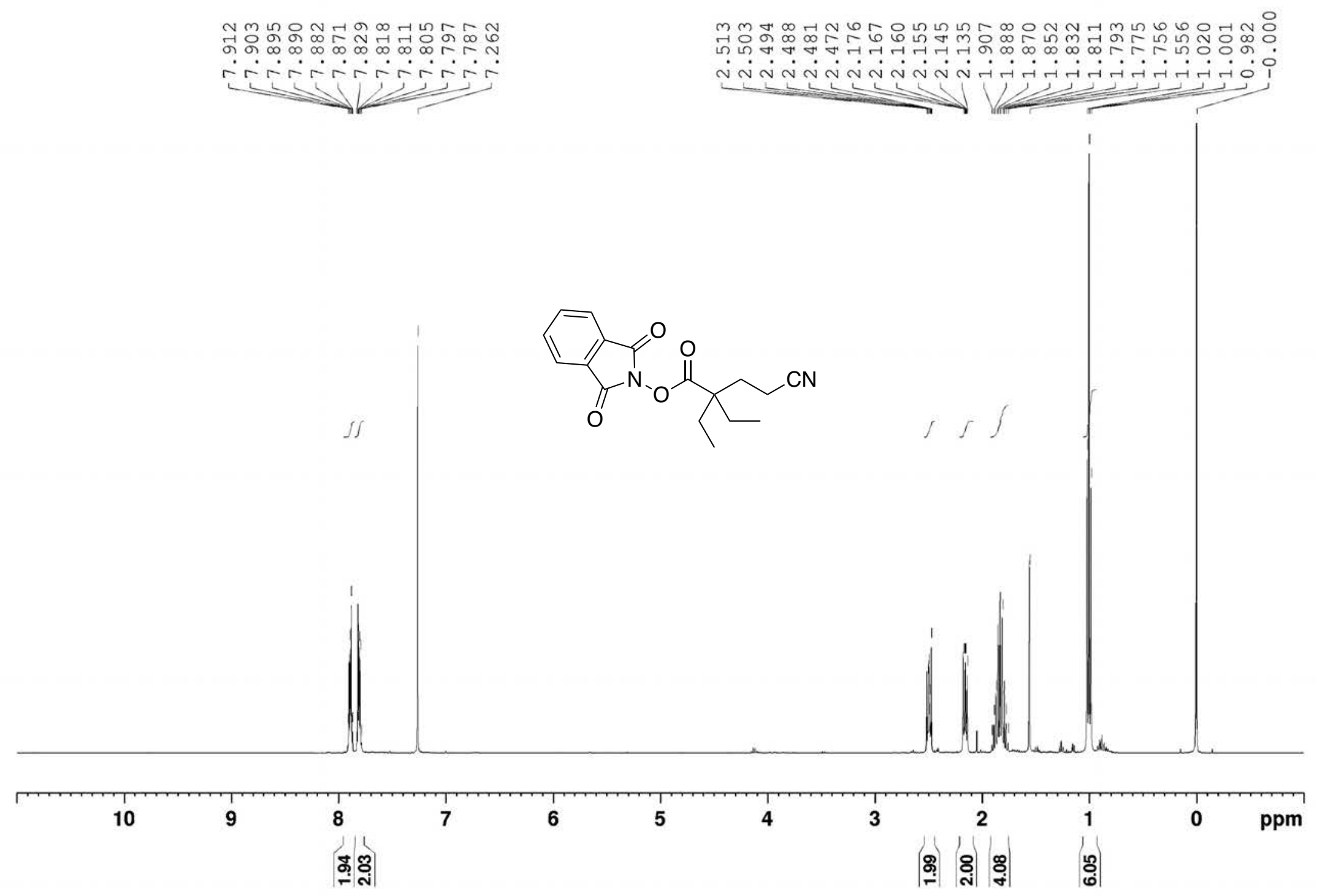

${ }^{1} \mathrm{H}$ NMR spectrum of $\mathbf{3 d}\left(400 \mathrm{MHz}, \mathrm{CDCl}_{3}\right)$ 


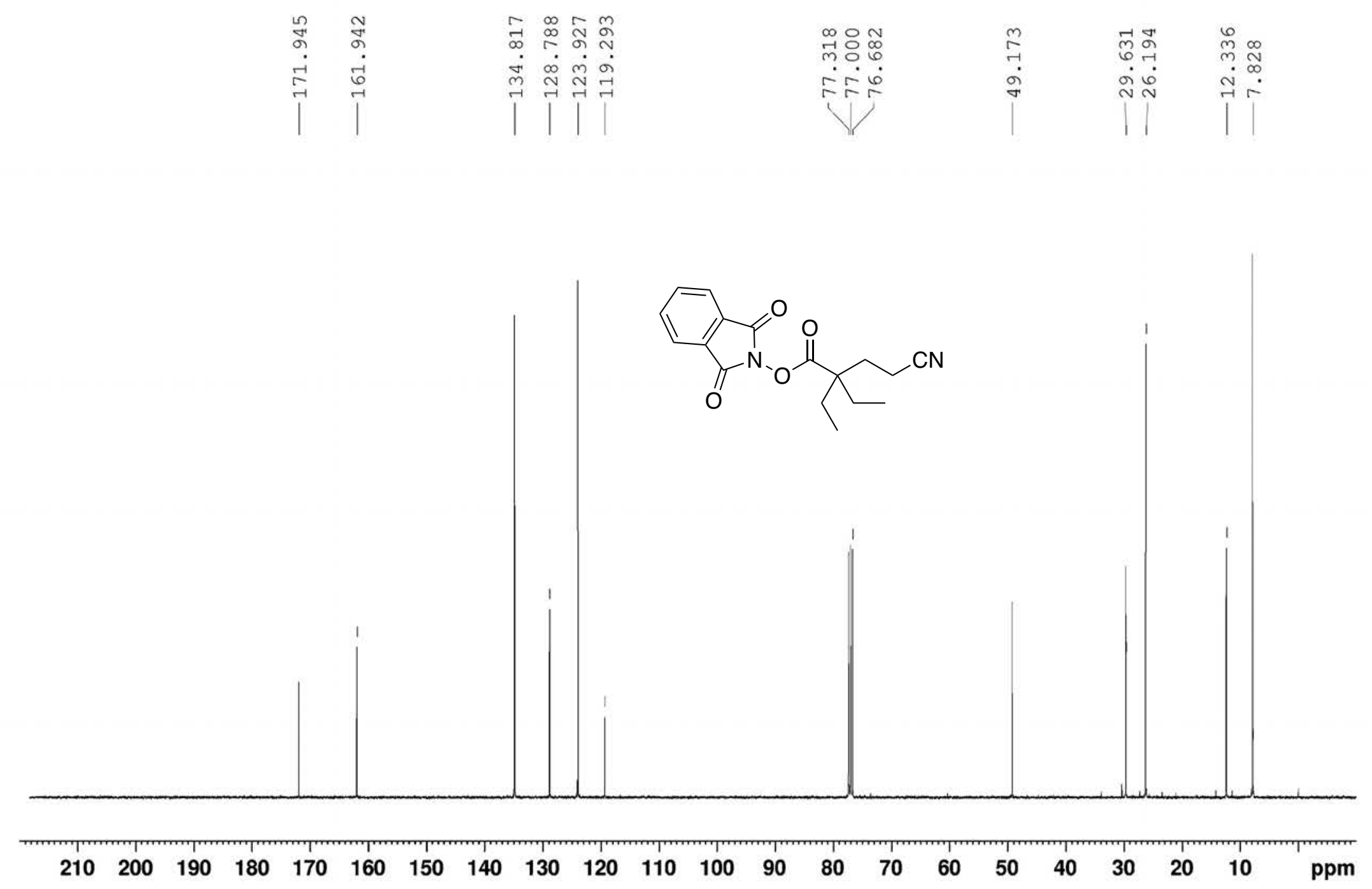

${ }^{13} \mathrm{C}$ NMR spectrum of $\mathbf{3 d}\left(100.6 \mathrm{MHz}, \mathrm{CDCl}_{3}\right)$ 


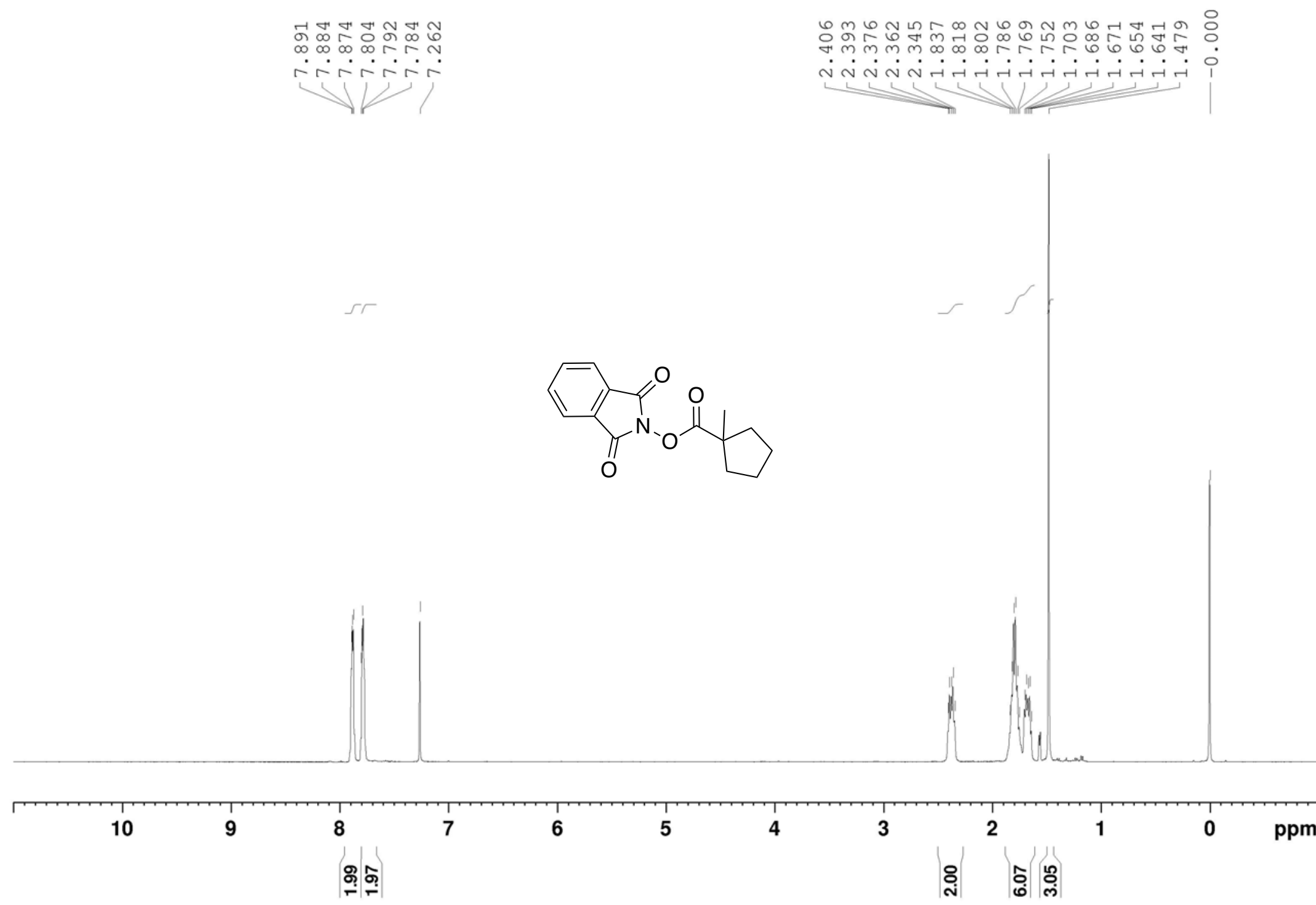

${ }^{1} \mathrm{H}$ NMR spectrum of $\mathbf{3 e}\left(400 \mathrm{MHz}, \mathrm{CDCl}_{3}\right)$ 


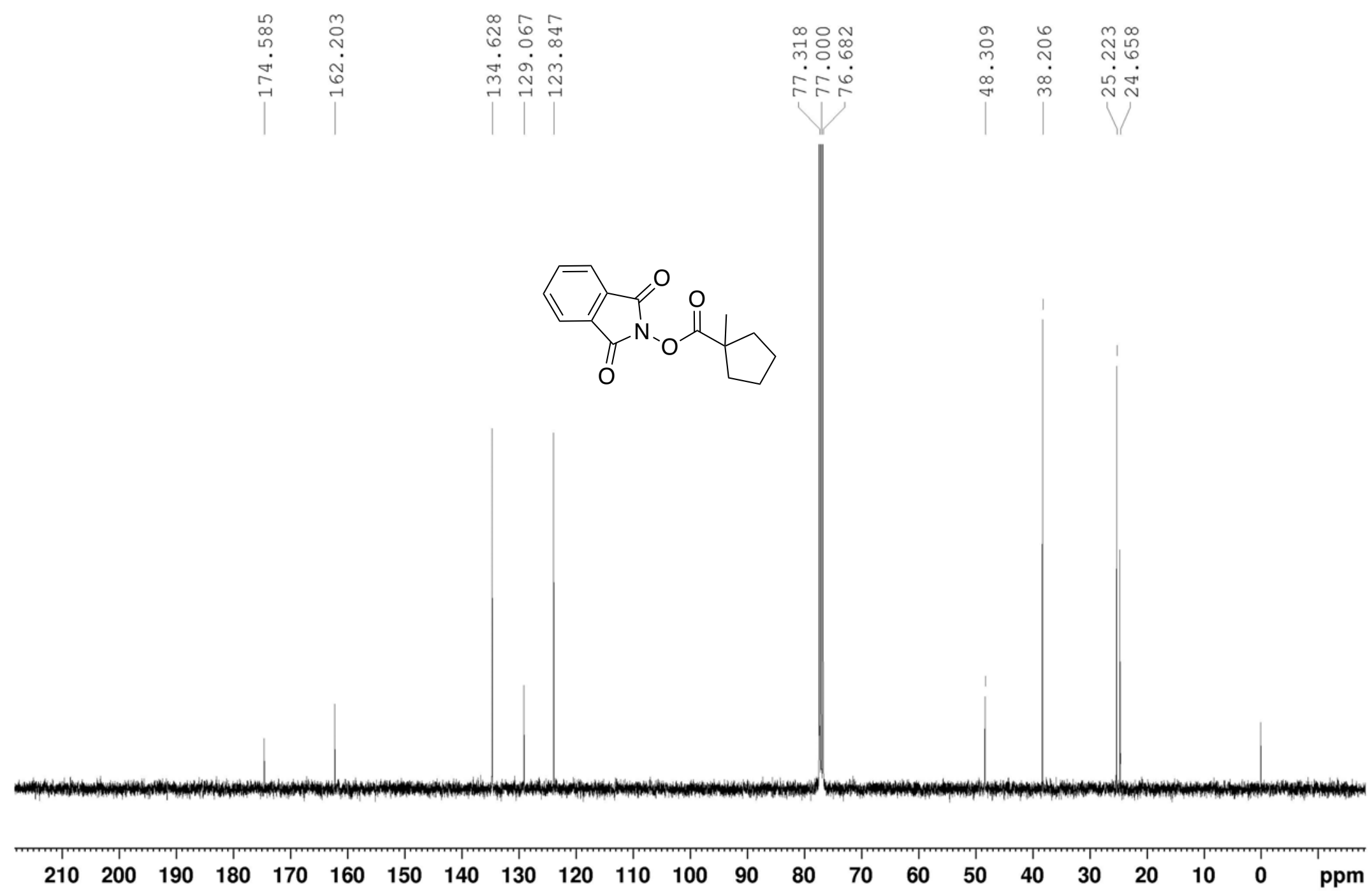

${ }^{13} \mathrm{C}$ NMR spectrum of $\mathbf{3 e}\left(100.6 \mathrm{MHz}, \mathrm{CDCl}_{3}\right)$ 


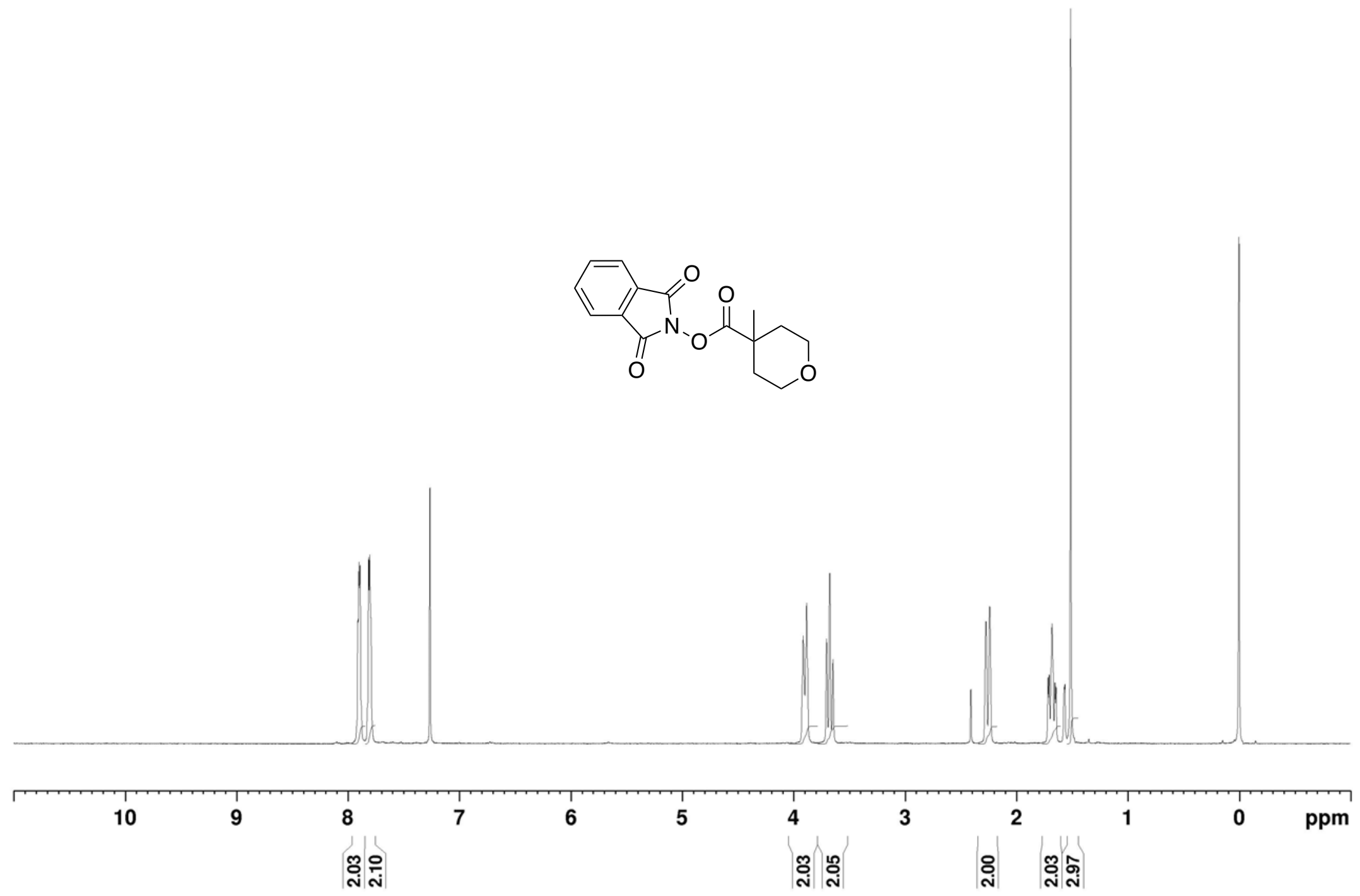

${ }^{1} \mathrm{H}$ NMR spectrum of $\mathbf{3 h}\left(400 \mathrm{MHz}, \mathrm{CDCl}_{3}\right)$ 

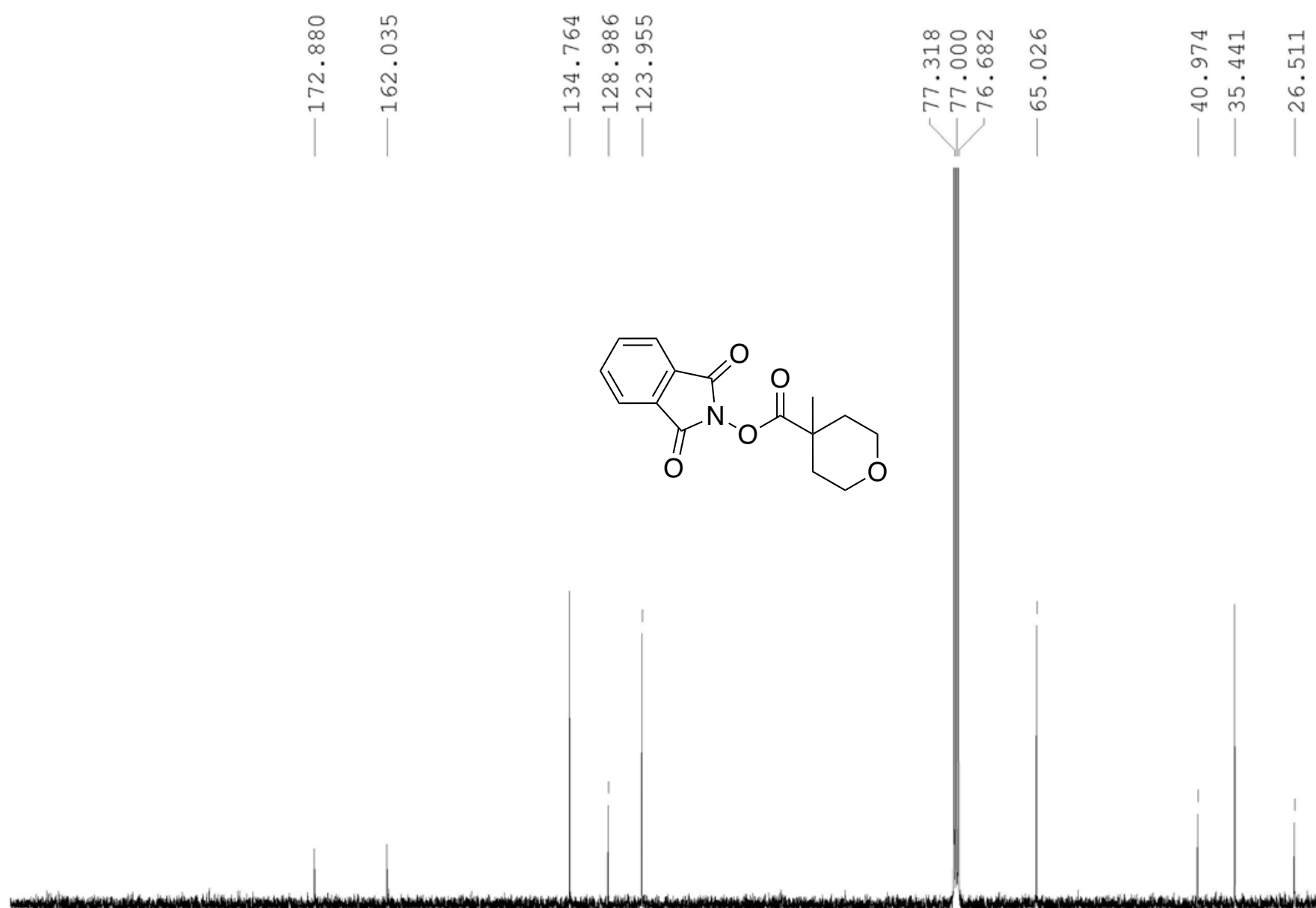

$\begin{array}{llllllllllllllllllllllll}210 & 200 & 190 & 180 & 170 & 160 & 150 & 140 & 130 & 120 & 110 & 100 & 90 & 80 & 70 & 60 & 50 & 40 & 30 & 20 & 10 & 0 & \text { ppm }\end{array}$

${ }^{13} \mathrm{C}$ NMR spectrum of $\mathbf{3 h}\left(100.6 \mathrm{MHz}, \mathrm{CDCl}_{3}\right)$ 


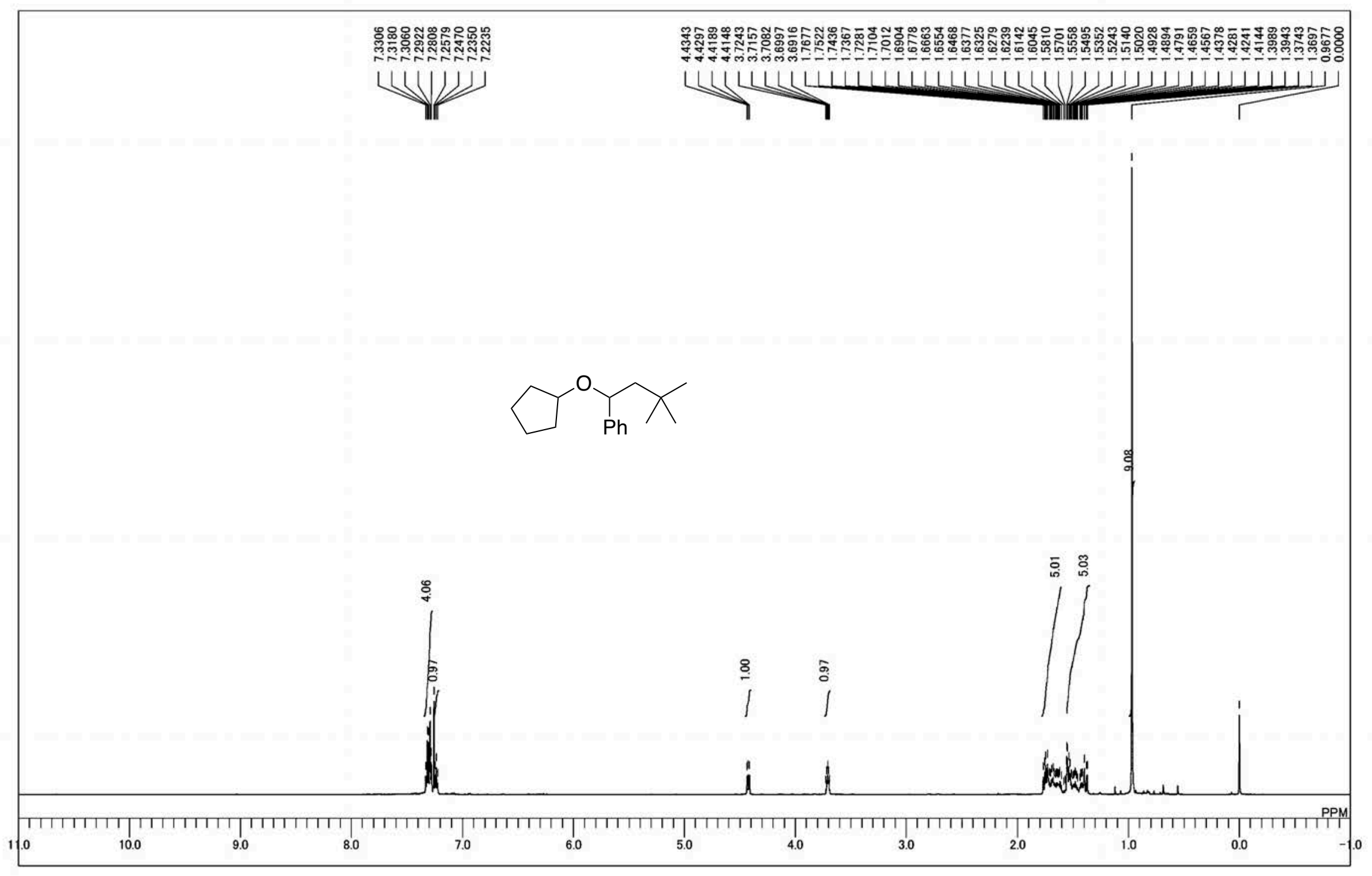

${ }^{1} \mathrm{H}$ NMR spectrum of 4 aaa $\left(600 \mathrm{MHz}, \mathrm{CDCl}_{3}\right)$ 


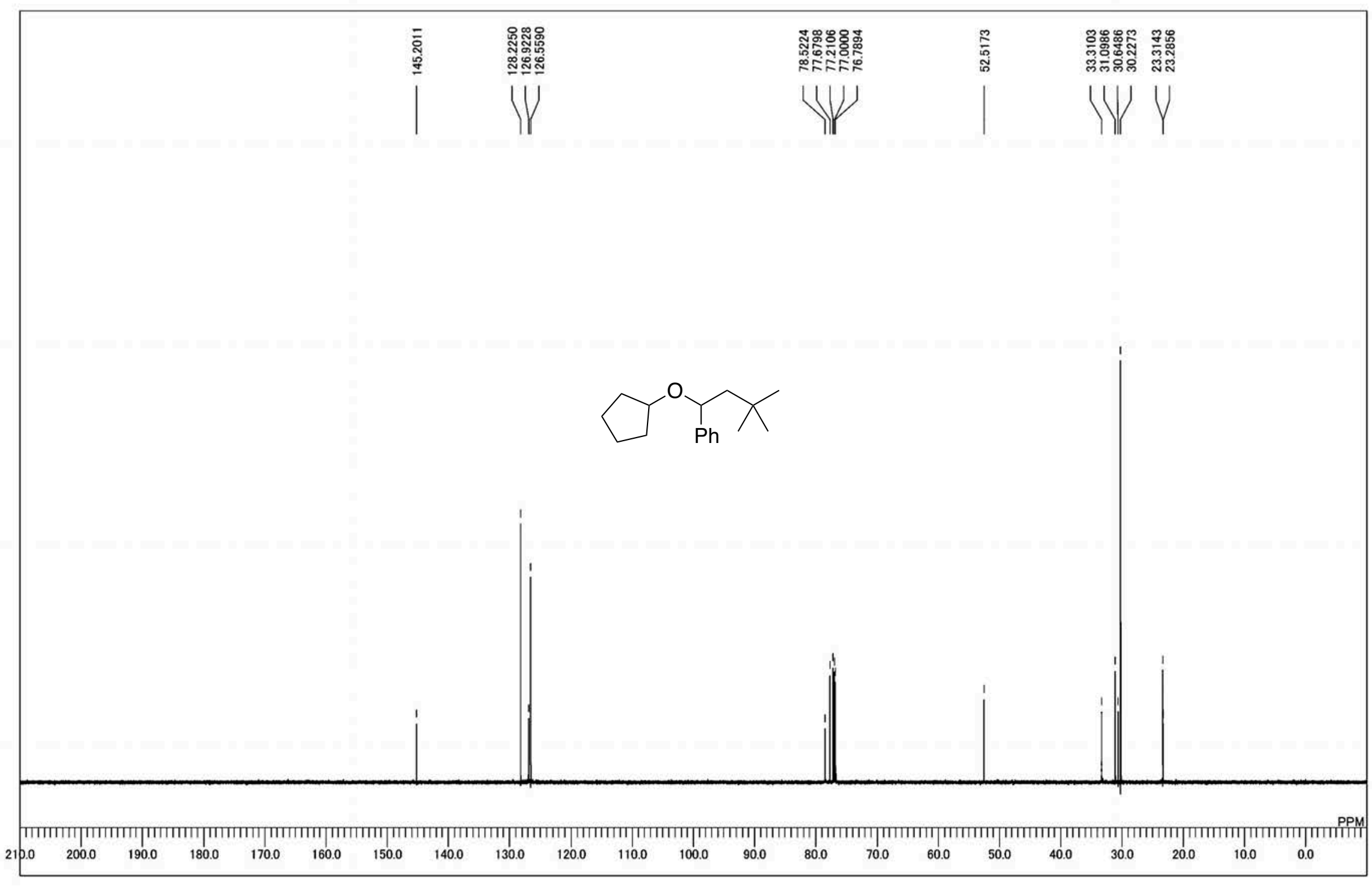

${ }^{13} \mathrm{C}$ NMR spectrum of 4 aaa $\left(150.9 \mathrm{MHz}, \mathrm{CDCl}_{3}\right)$ 


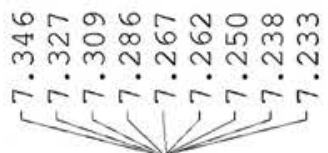

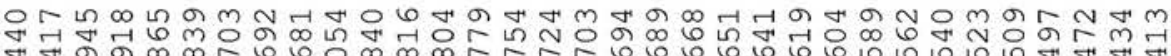

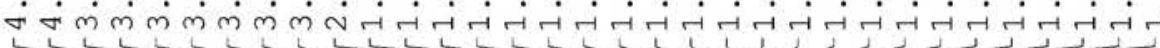
एकी

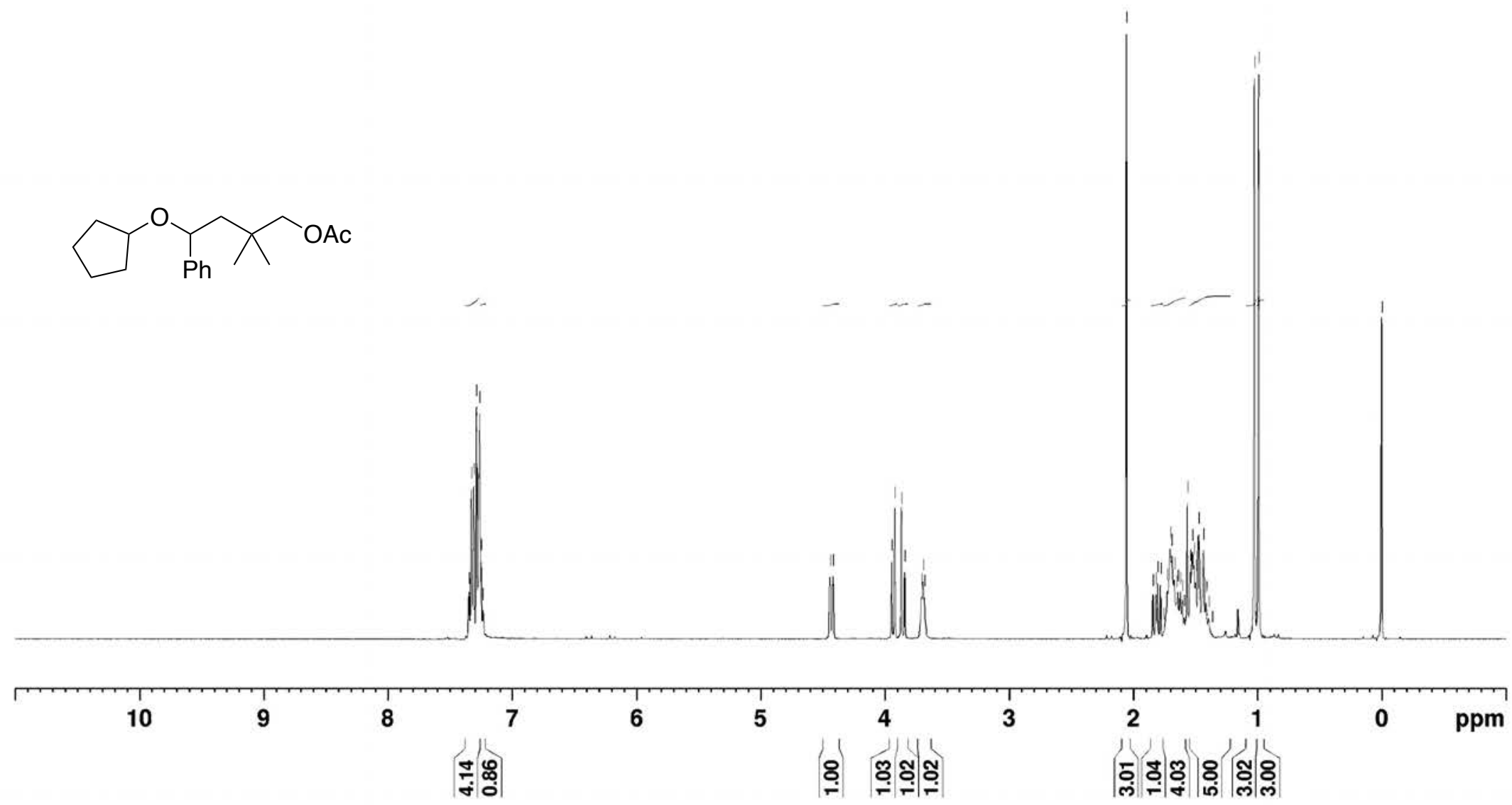

${ }^{1} \mathrm{H}$ NMR spectrum of $\mathbf{4 a a b}\left(400 \mathrm{MHz}, \mathrm{CDCl}_{3}\right)$ 


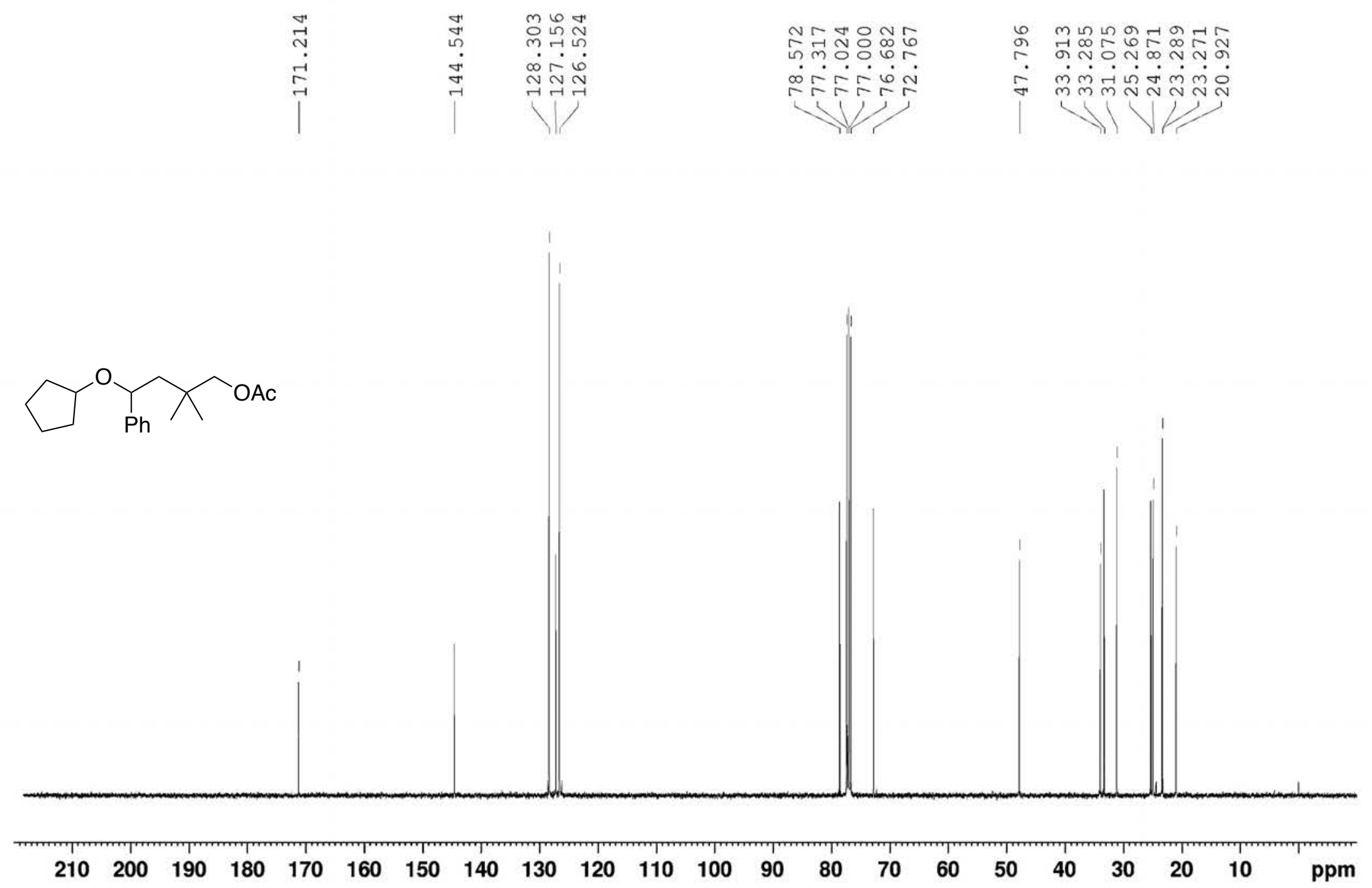

${ }^{13} \mathrm{C}$ NMR spectrum of $\mathbf{4 a a b}\left(100.6 \mathrm{MHz}, \mathrm{CDCl}_{3}\right)$ 


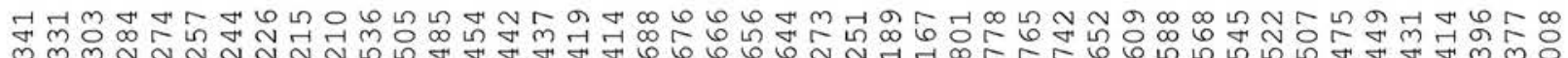

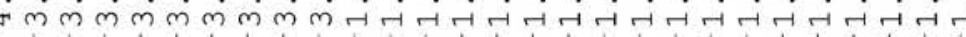

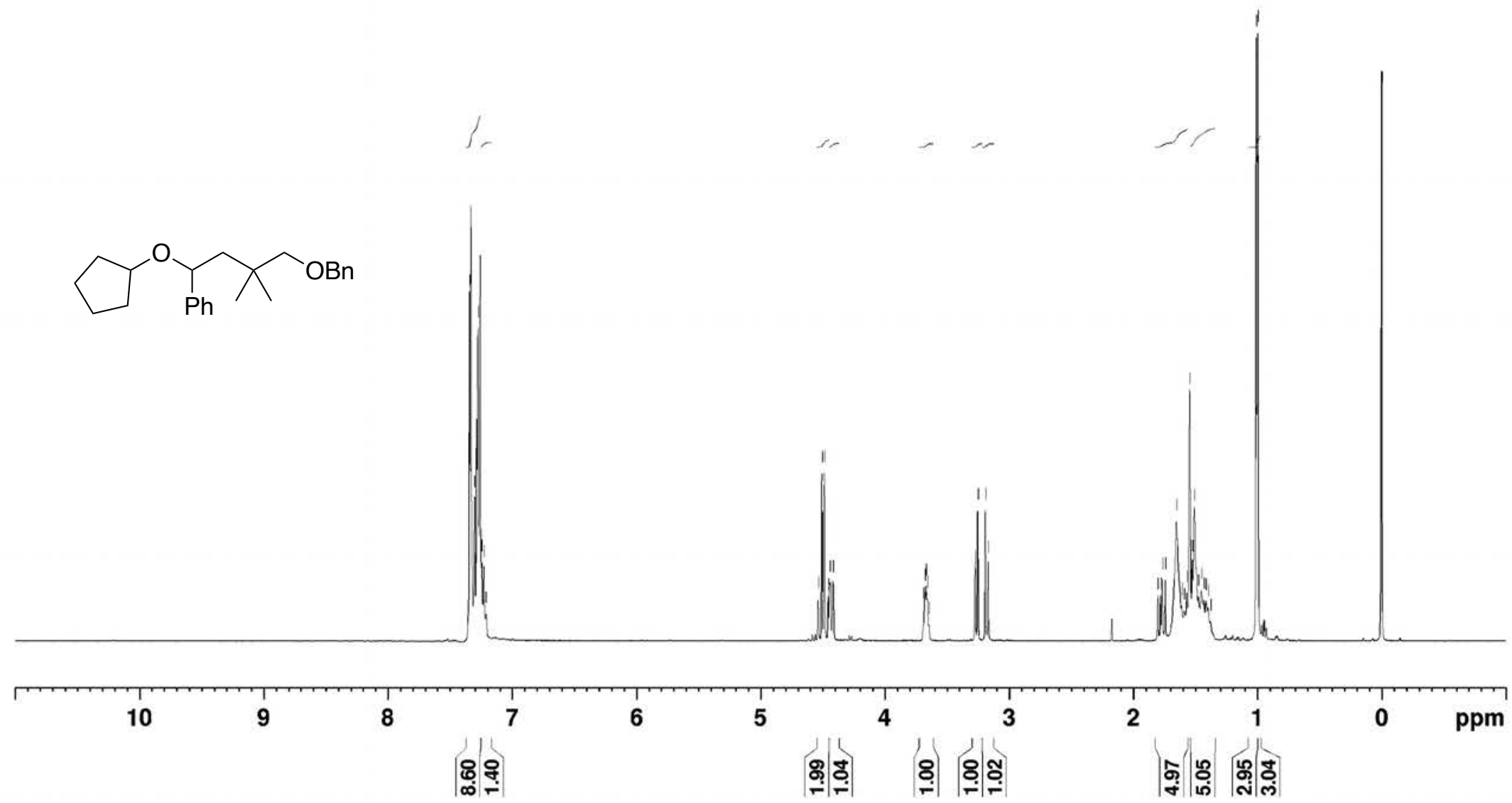

${ }^{1} \mathrm{H}$ NMR spectrum of $4 \mathbf{a a c}\left(400 \mathrm{MHz}, \mathrm{CDCl}_{3}\right)$ 


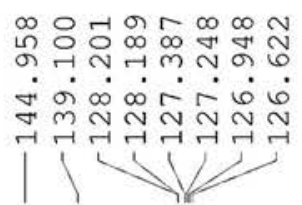

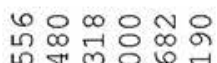

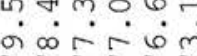

$\longrightarrow \mathrm{OBn}$ $\mathrm{Ph}$

$\mathrm{Bn}$
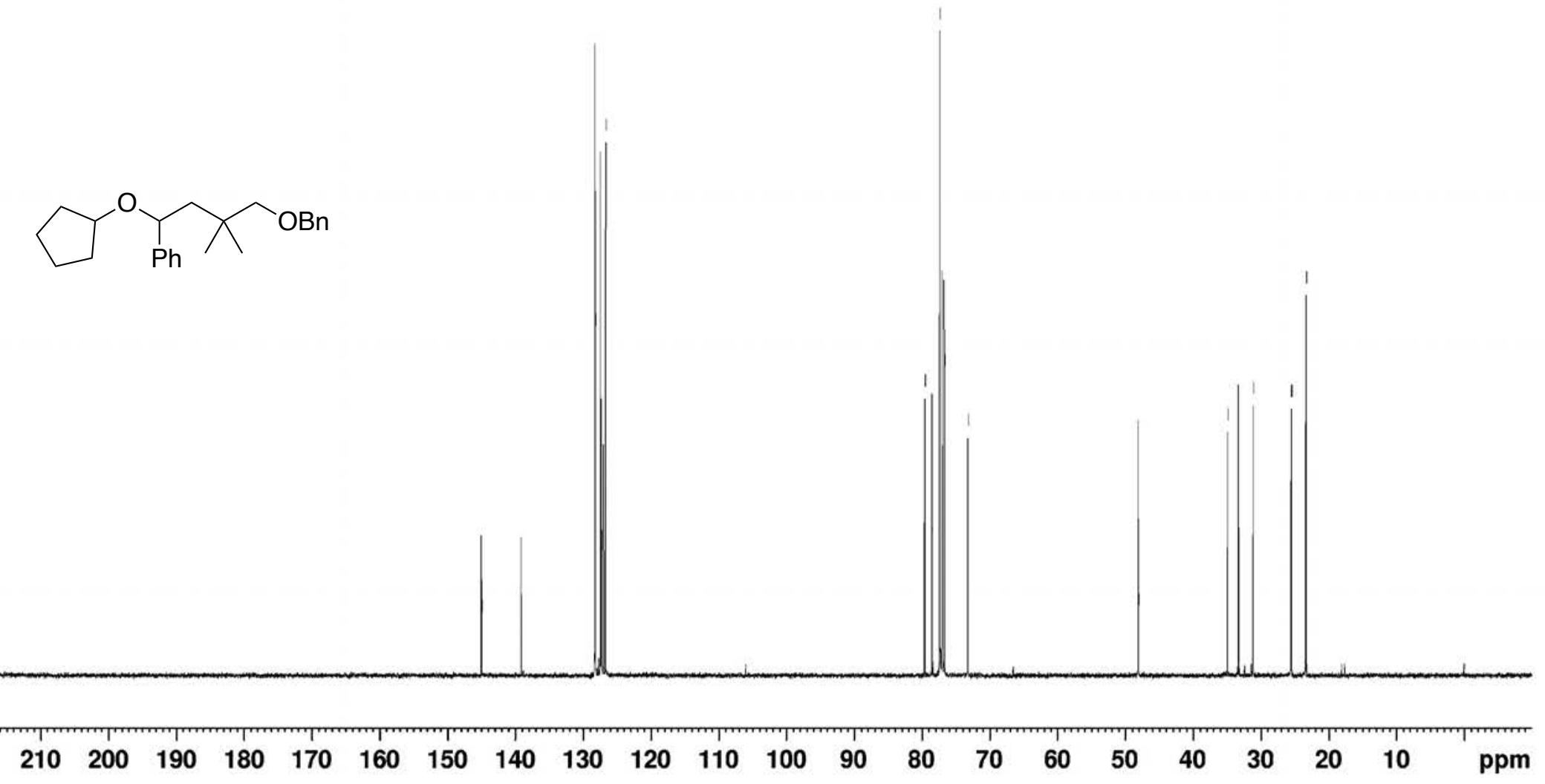

${ }^{13} \mathrm{C}$ NMR spectrum of 4 aac $\left(100.6 \mathrm{MHz}, \mathrm{CDCl}_{3}\right)$ 


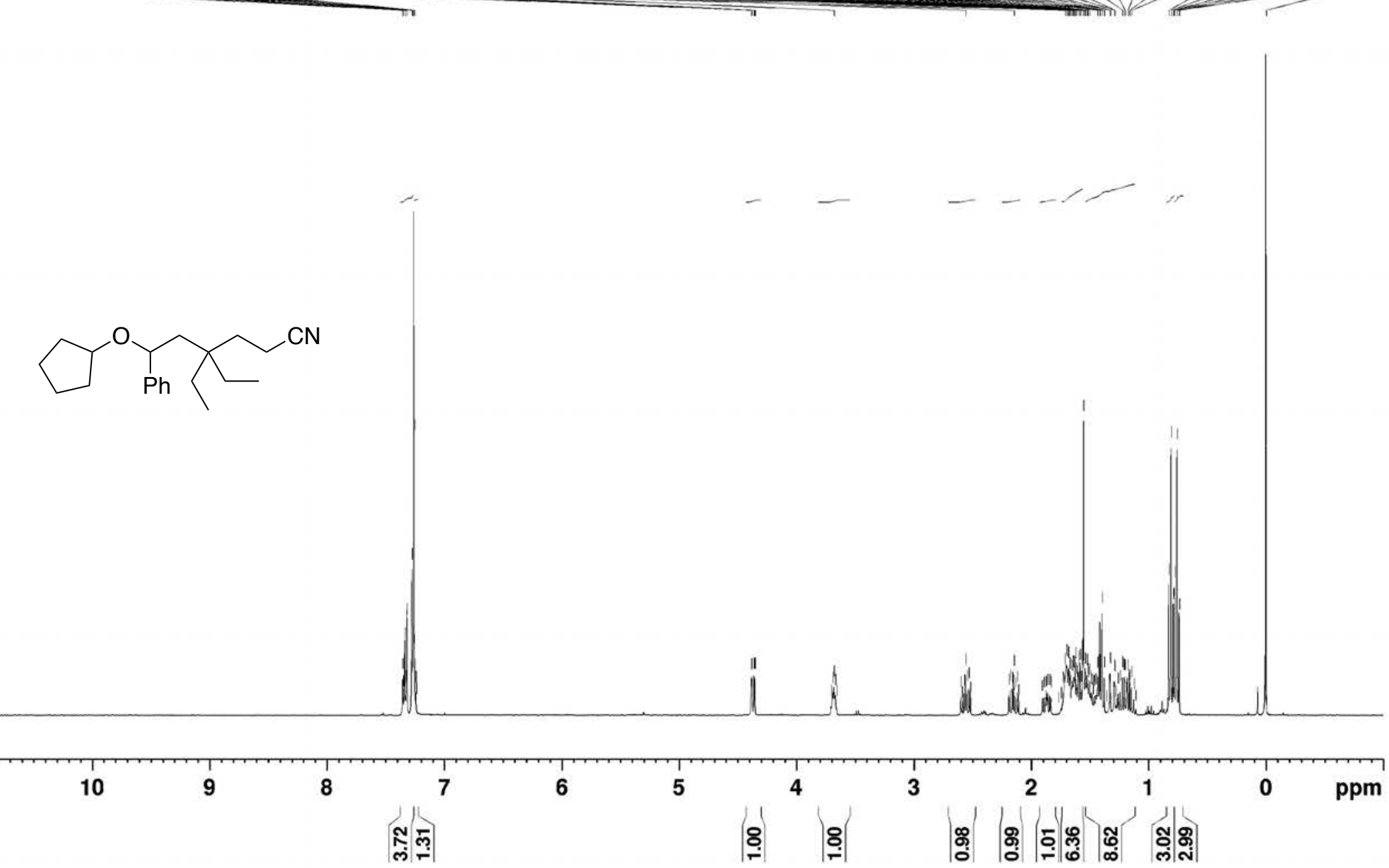

${ }^{1} \mathrm{H}$ NMR spectrum of $\mathbf{4 a a d}\left(400 \mathrm{MHz}, \mathrm{CDCl}_{3}\right)$ 


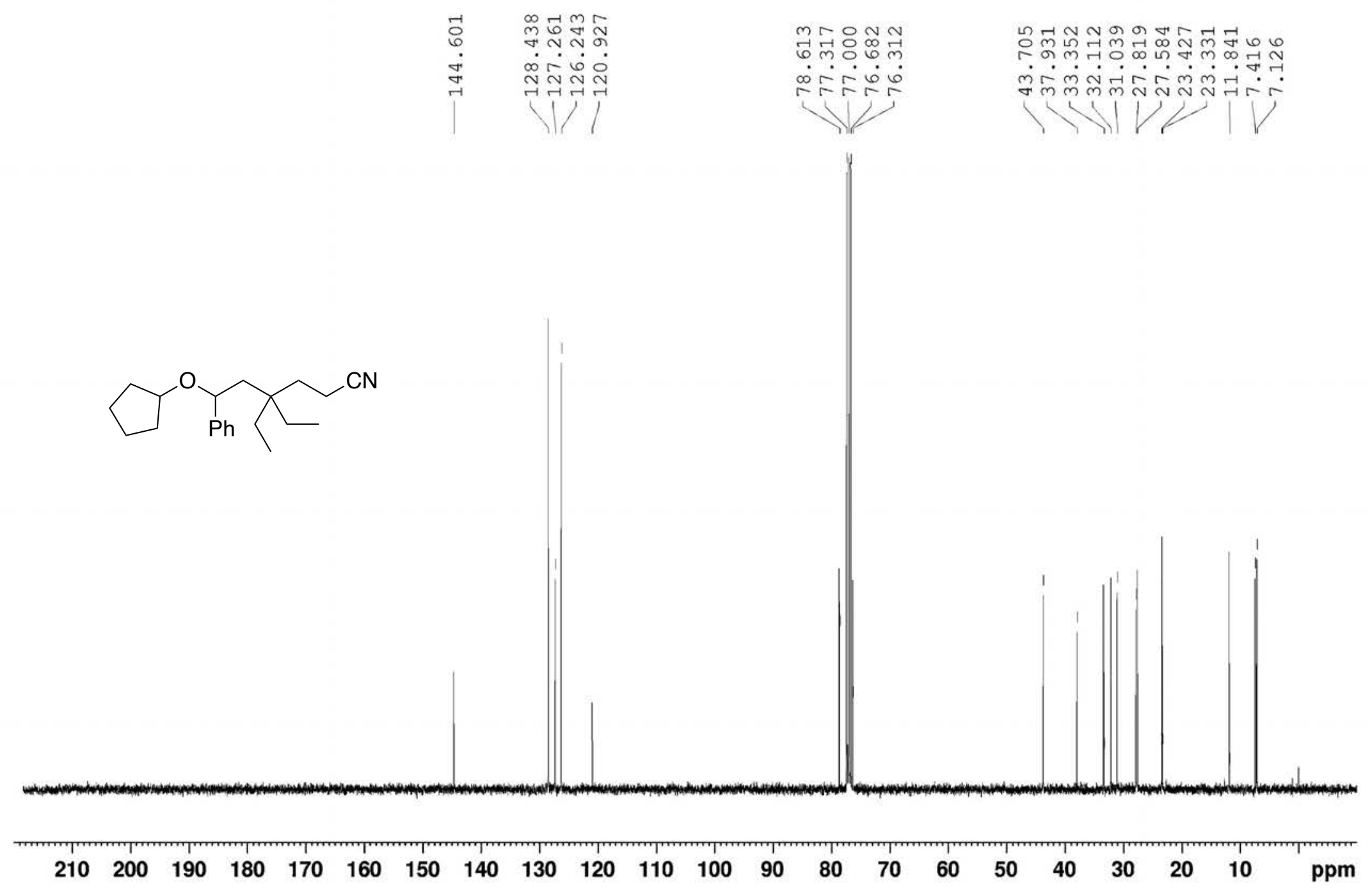

${ }^{13} \mathrm{C}$ NMR spectrum of $\mathbf{4 a a d}\left(100.6 \mathrm{MHz}, \mathrm{CDCl}_{3}\right)$ 


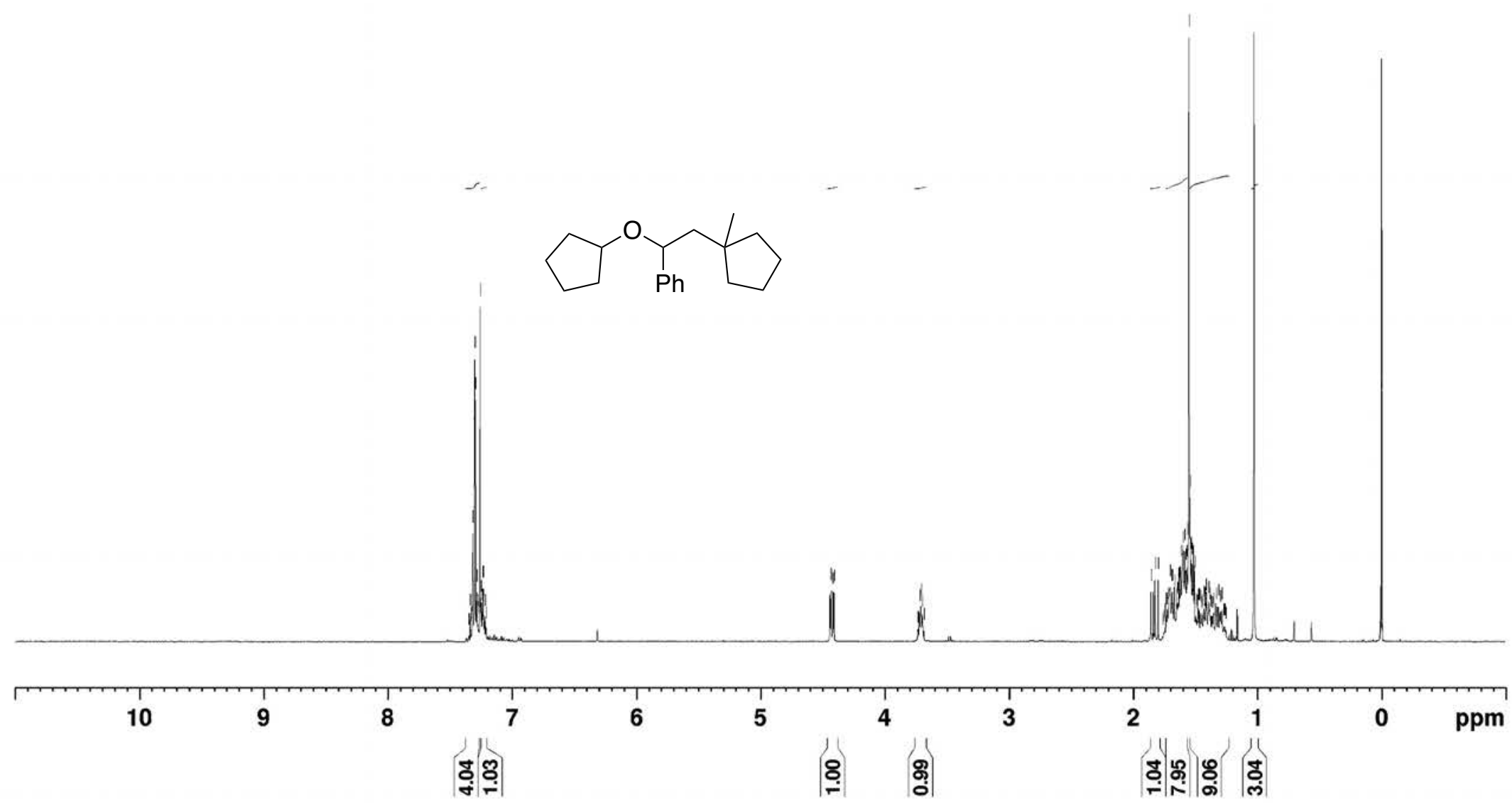

${ }^{1} \mathrm{H}$ NMR spectrum of 4 aae $\left(400 \mathrm{MHz}, \mathrm{CDCl}_{3}\right)$ 


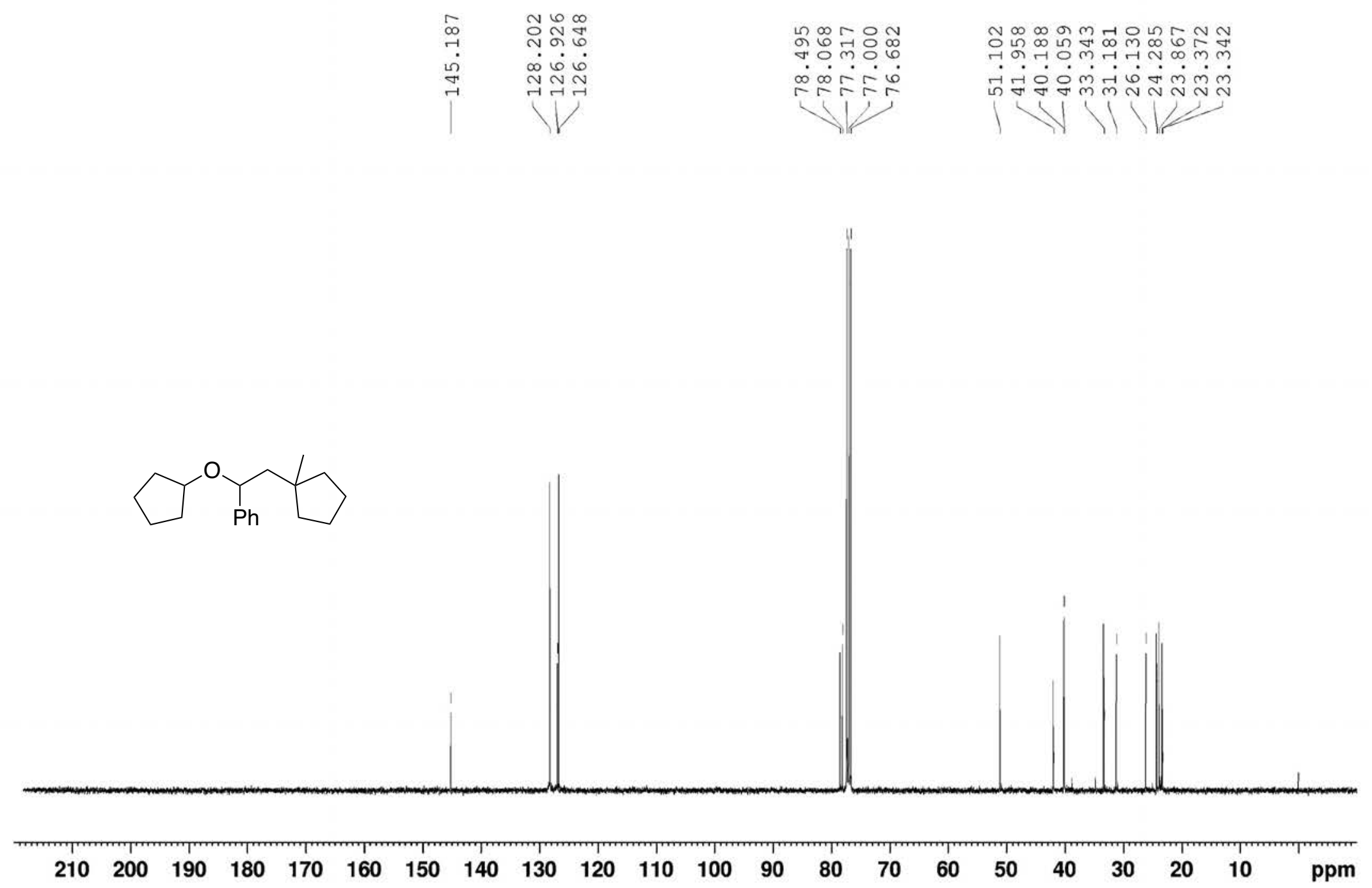

${ }^{13} \mathrm{C}$ NMR spectrum of 4 aae $\left(100.6 \mathrm{MHz}, \mathrm{CDCl}_{3}\right)$ 


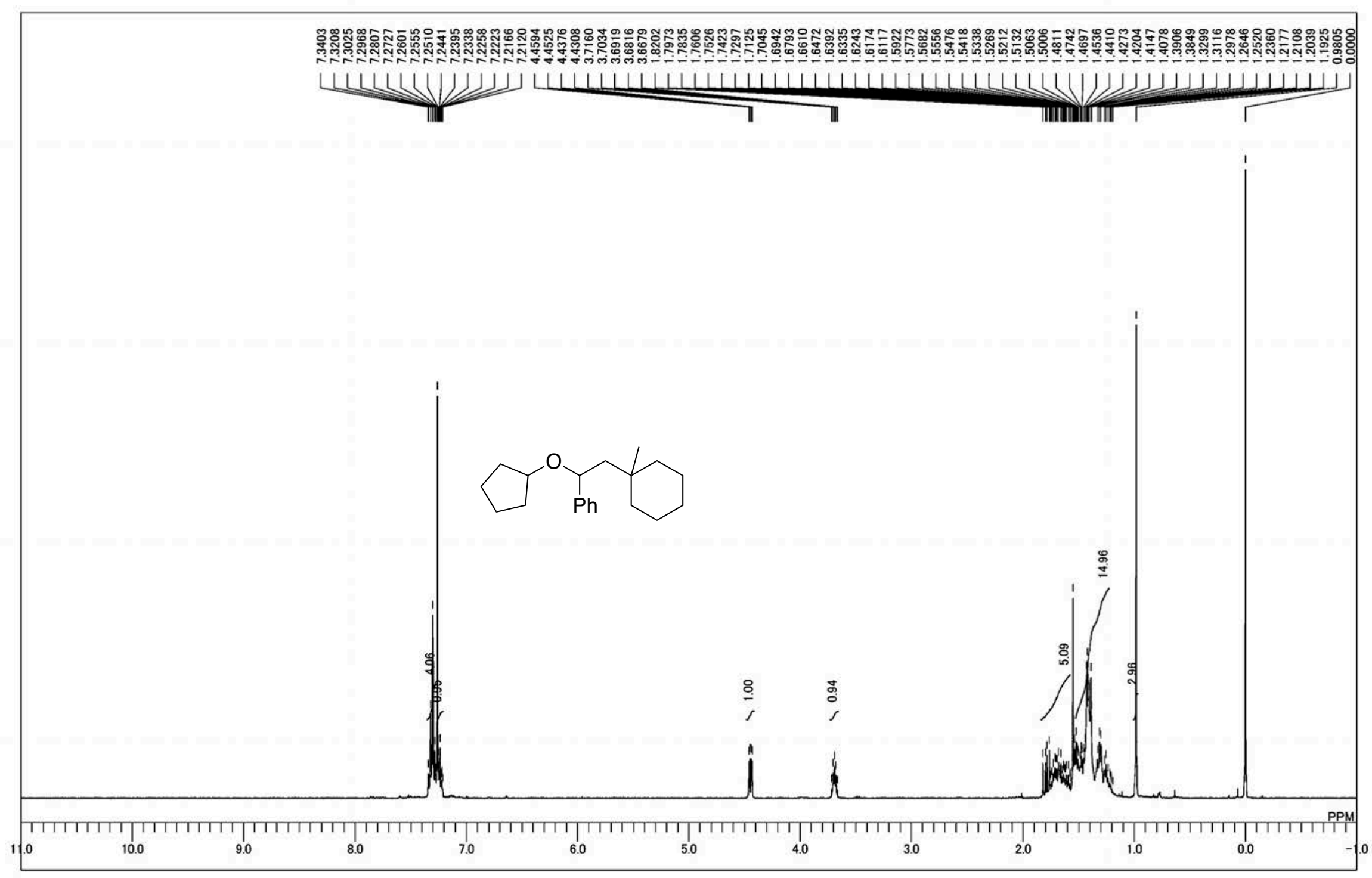

${ }^{1} \mathrm{H}$ NMR spectrum of 4 aaf $\left(400 \mathrm{MHz}, \mathrm{CDCl}_{3}\right)$ 


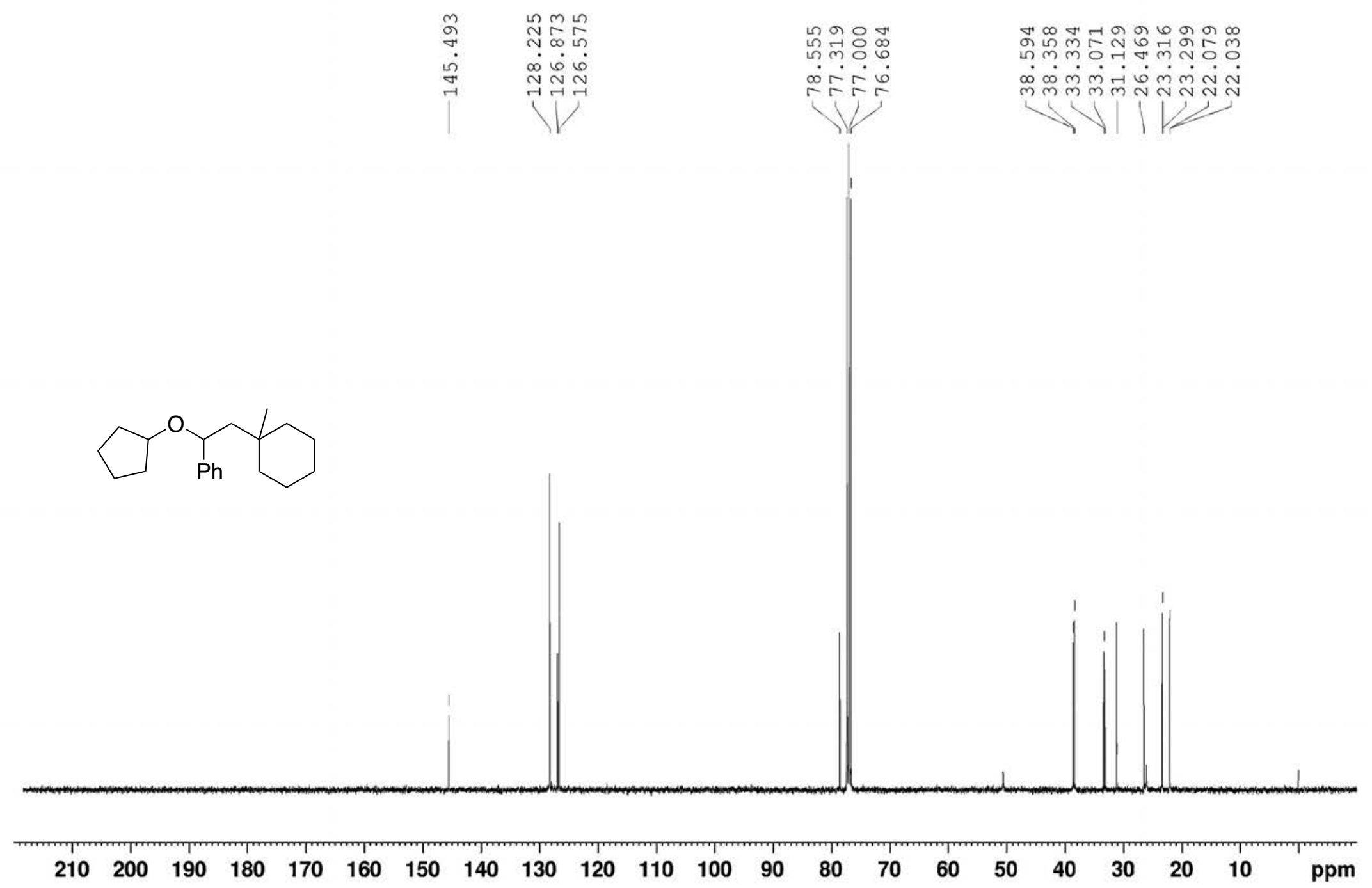

${ }^{13} \mathrm{C}$ NMR spectrum of 4 aaf $\left(100.6 \mathrm{MHz}, \mathrm{CDCl}_{3}\right)$ 


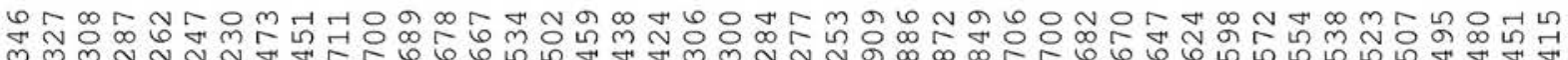
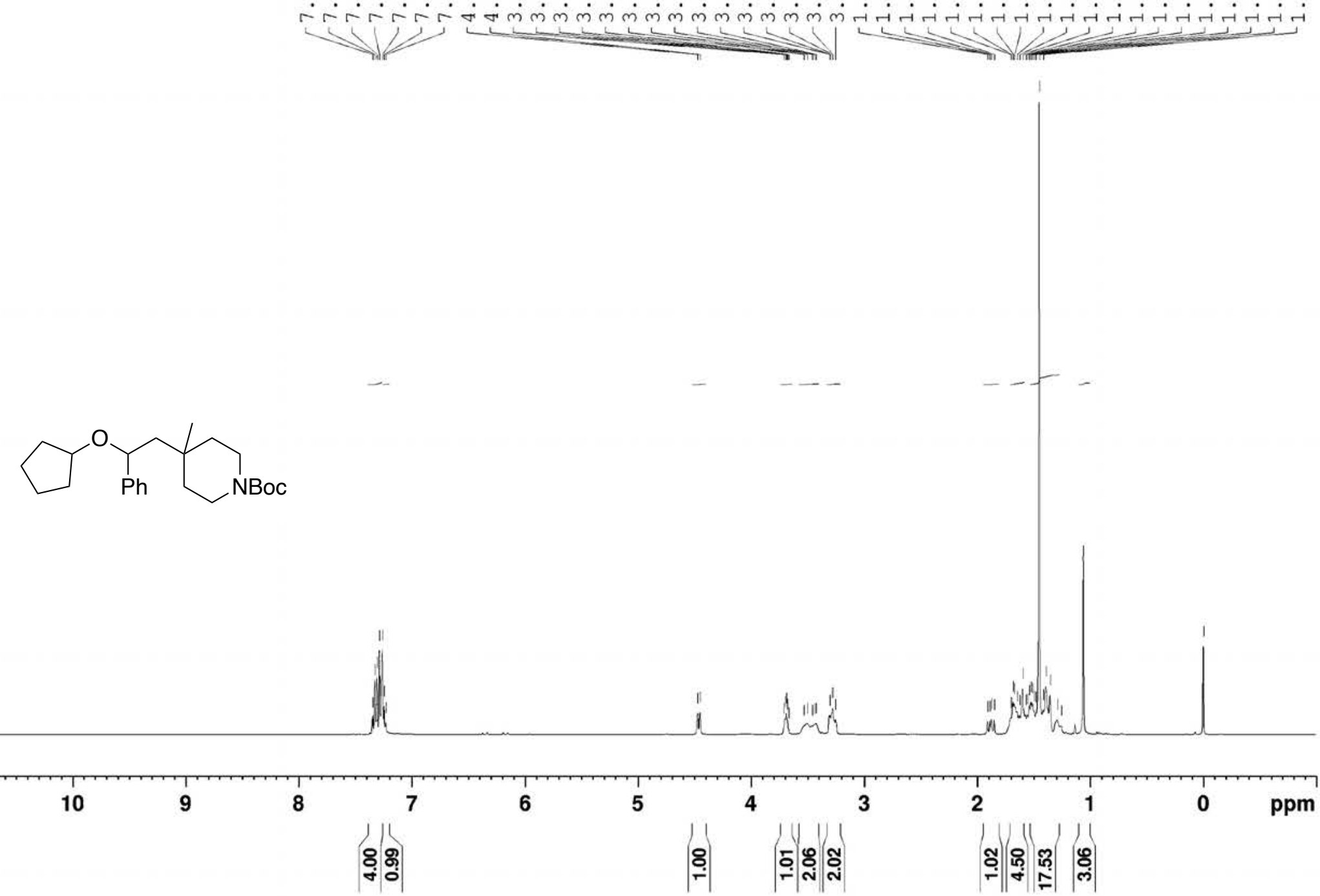

${ }^{1} \mathrm{H}$ NMR spectrum of $\mathbf{4 a a g}\left(400 \mathrm{MHz}, \mathrm{CDCl}_{3}\right)$ 


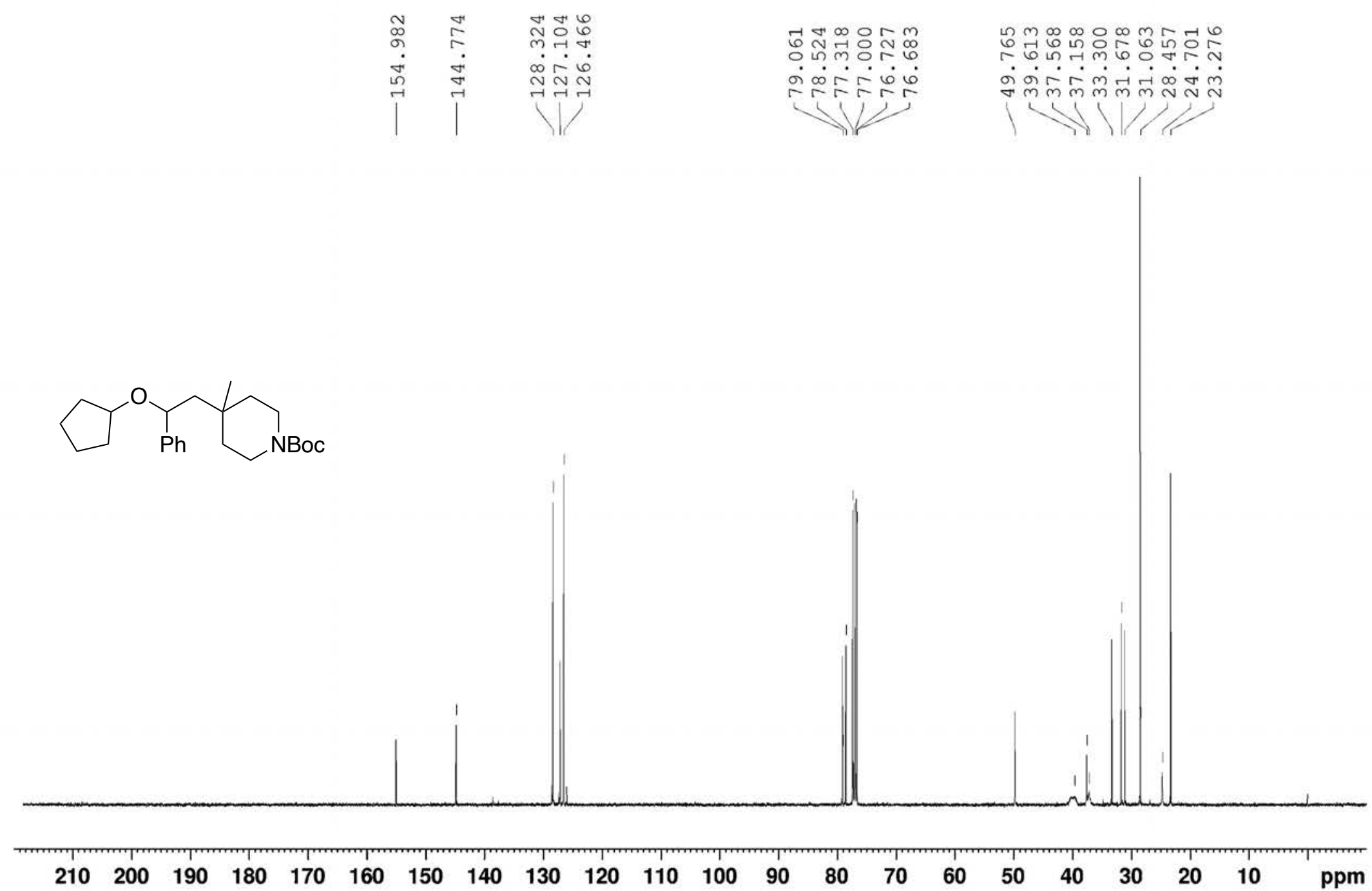

${ }^{13} \mathrm{C}$ NMR spectrum of $\mathbf{4 a a g}\left(100.6 \mathrm{MHz}, \mathrm{CDCl}_{3}\right)$ 


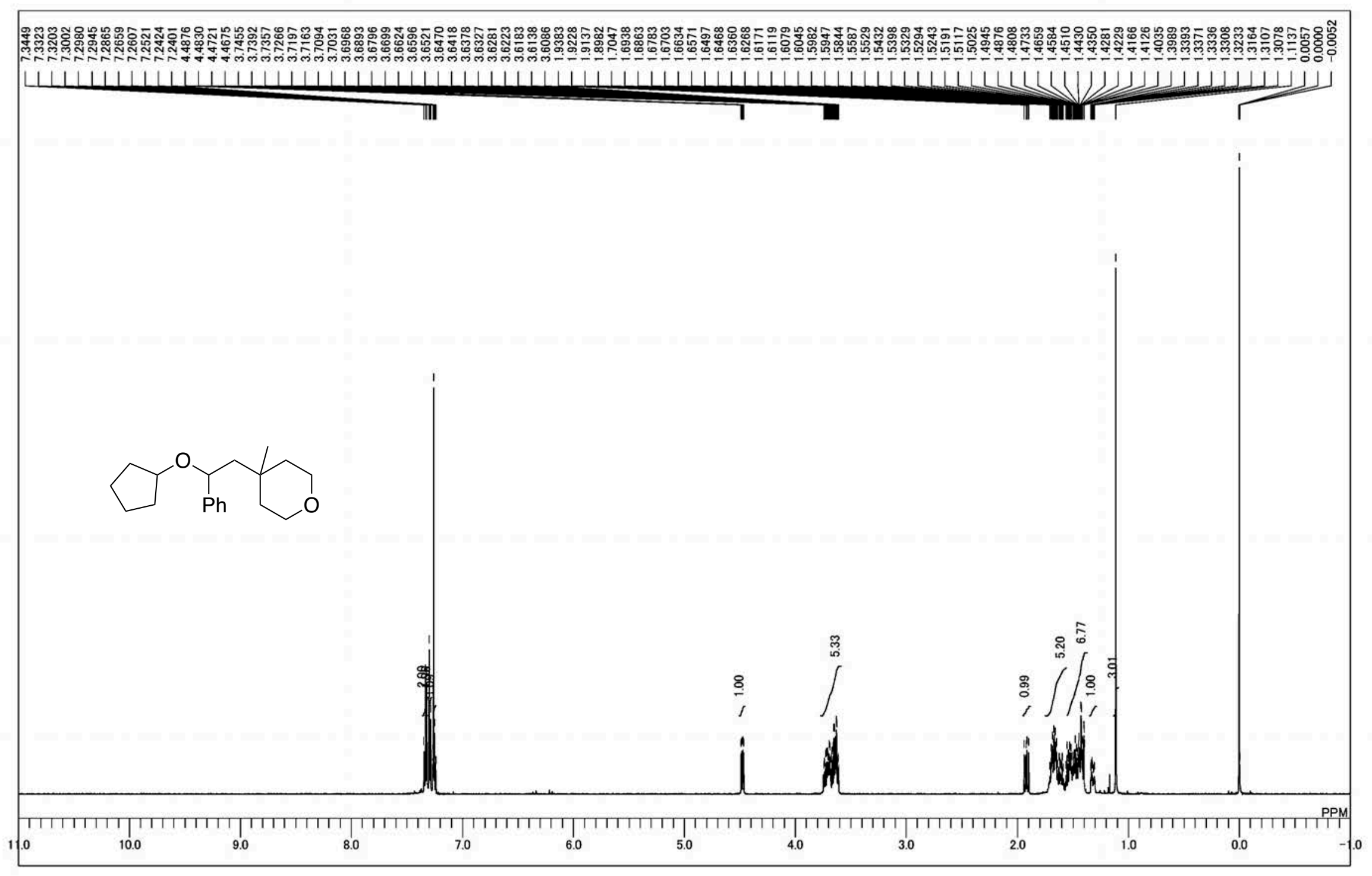

${ }^{1} \mathrm{H}$ NMR spectrum of $\mathbf{4 a a h}\left(600 \mathrm{MHz}, \mathrm{CDCl}_{3}\right)$ 


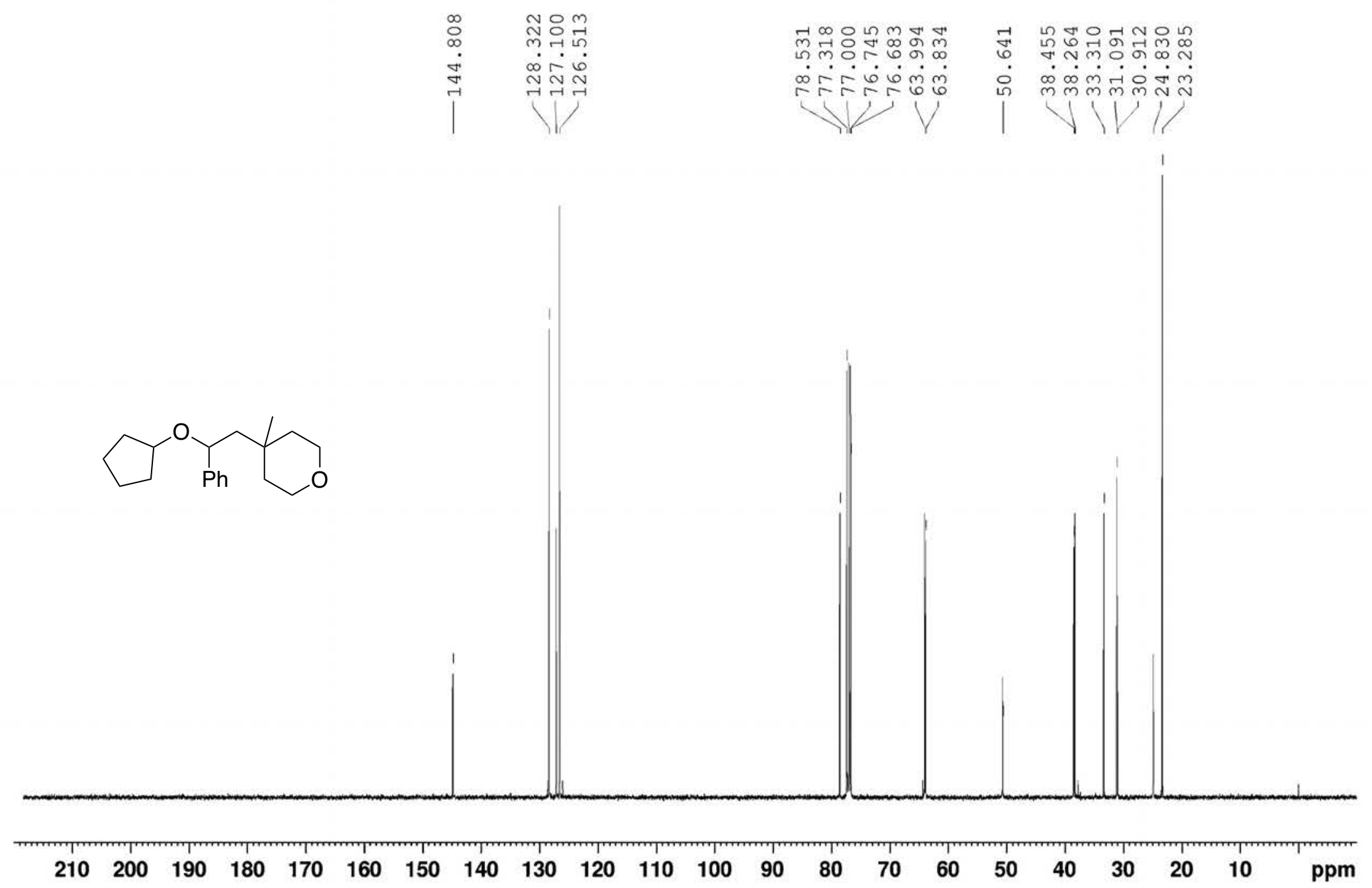

${ }^{13} \mathrm{C}$ NMR spectrum of $\mathbf{4 a a h}\left(100.6 \mathrm{MHz}, \mathrm{CDCl}_{3}\right)$ 


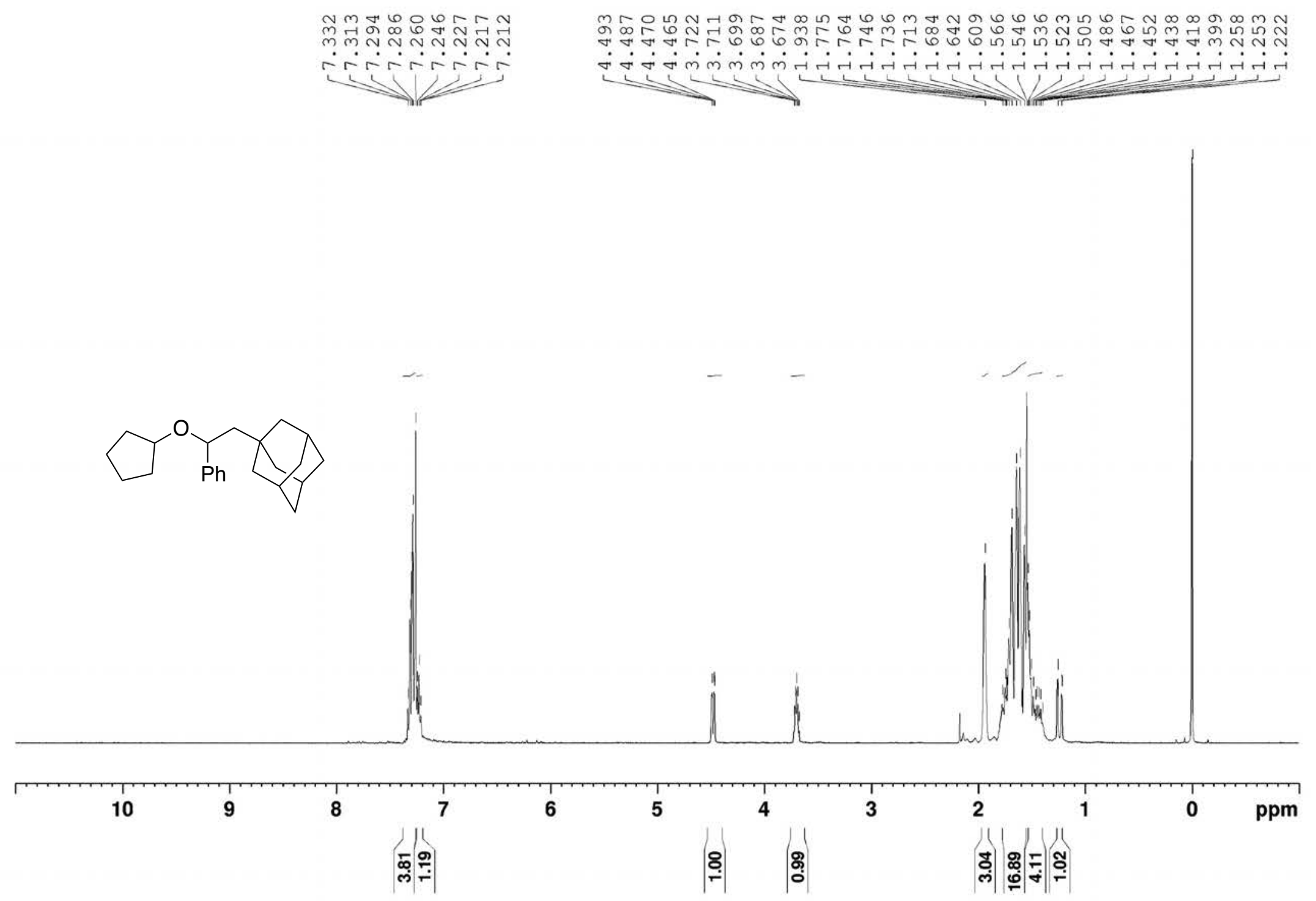

${ }^{1} \mathrm{H}$ NMR spectrum of $4 \mathbf{a a i}\left(400 \mathrm{MHz}, \mathrm{CDCl}_{3}\right)$ 


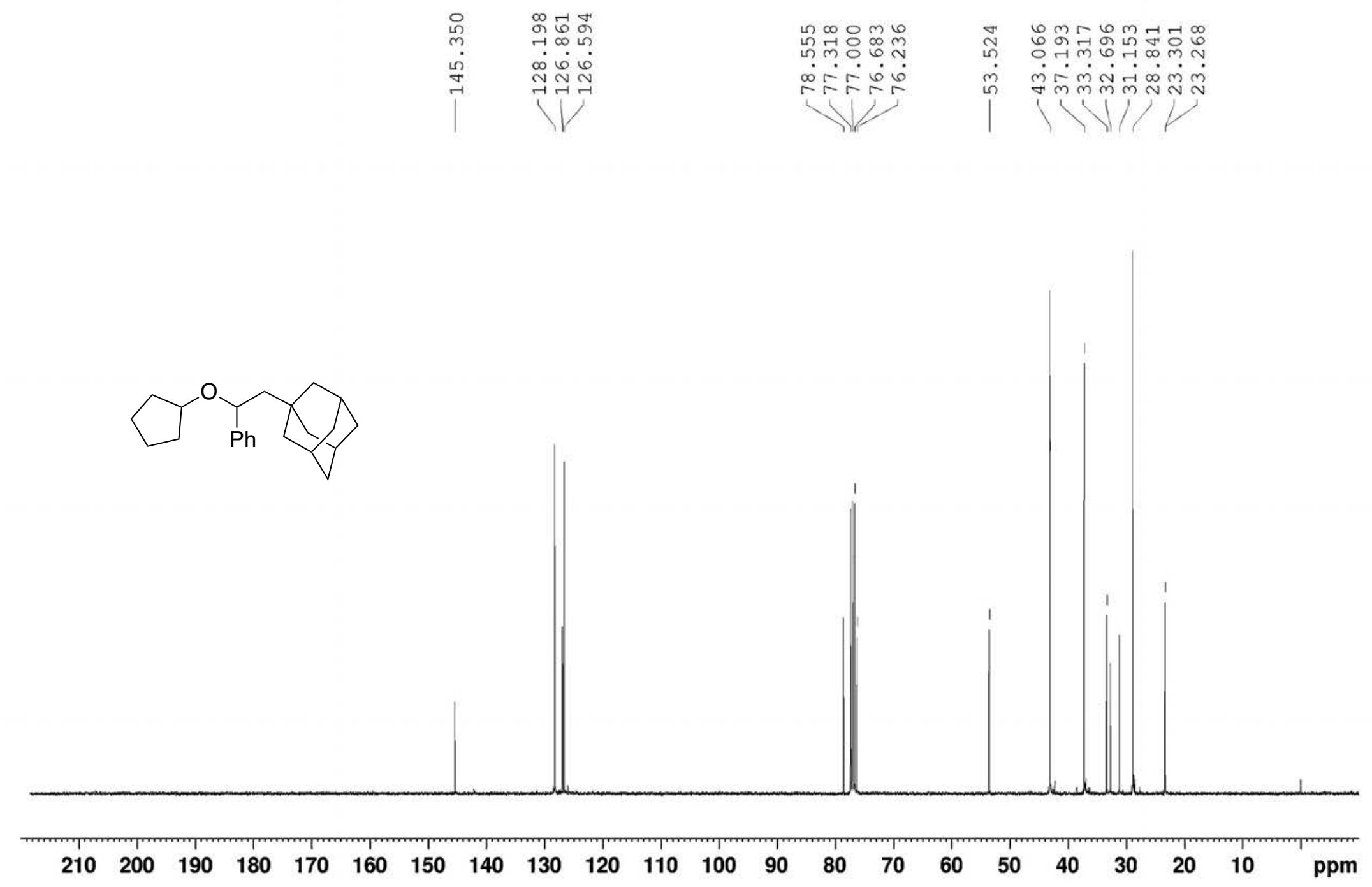

${ }^{13} \mathrm{C}$ NMR spectrum of 4 aai $\left(100.6 \mathrm{MHz}, \mathrm{CDCl}_{3}\right)$ 


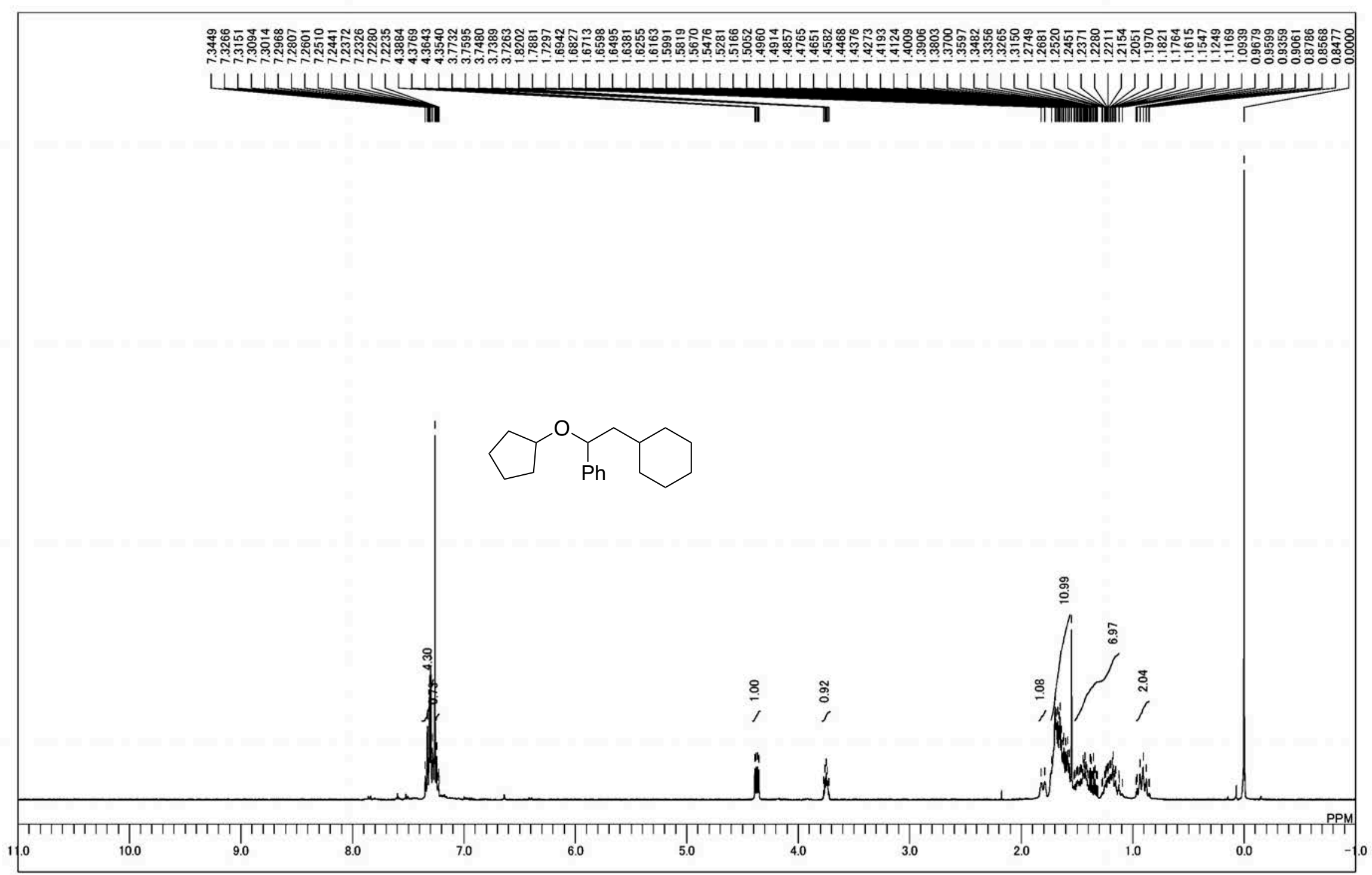

${ }^{1} \mathrm{H}$ NMR spectrum of $4 \mathbf{a a j}\left(400 \mathrm{MHz}, \mathrm{CDCl}_{3}\right)$ 


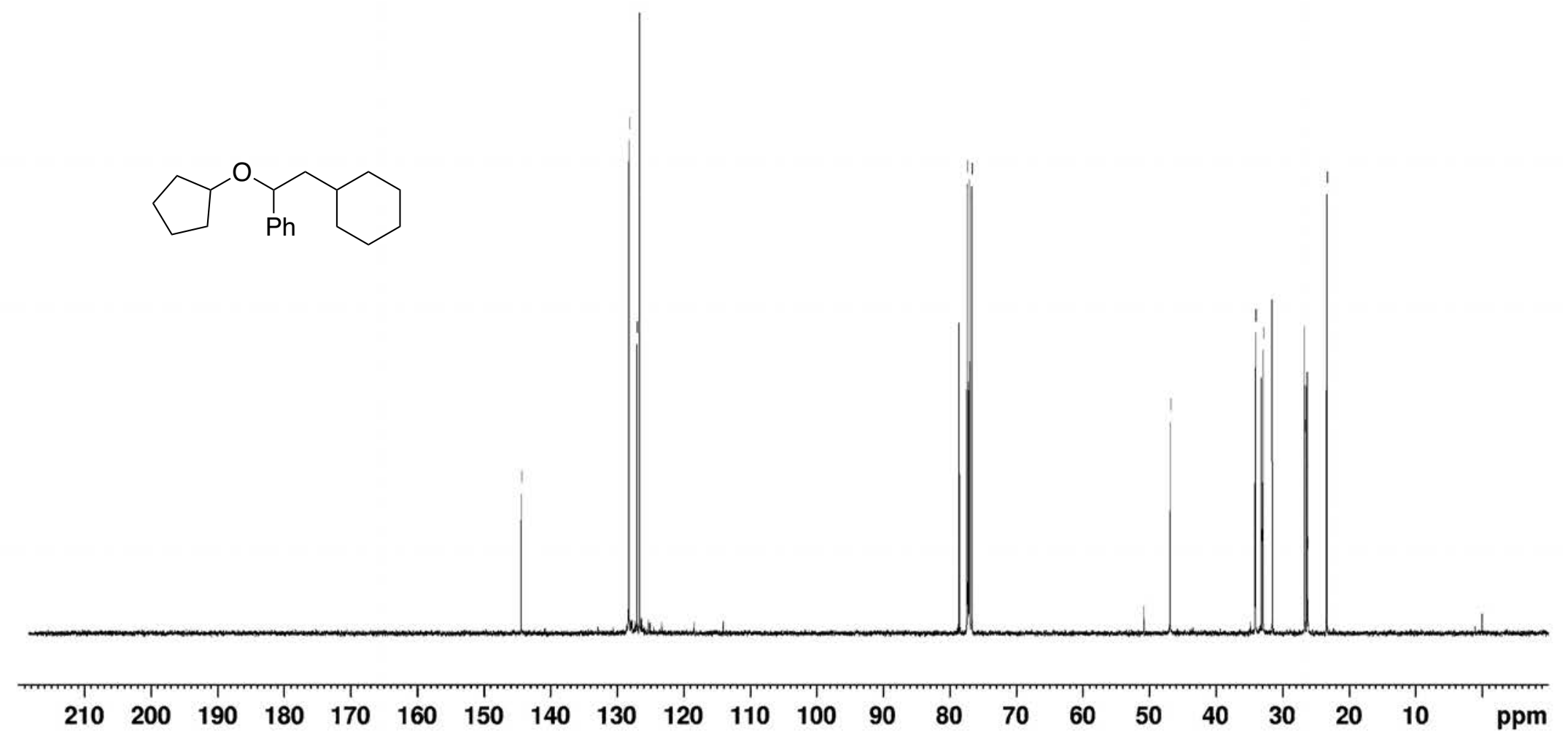

${ }^{13} \mathrm{C}$ NMR spectrum of 4 aaj $\left(100.6 \mathrm{MHz}, \mathrm{CDCl}_{3}\right)$ 


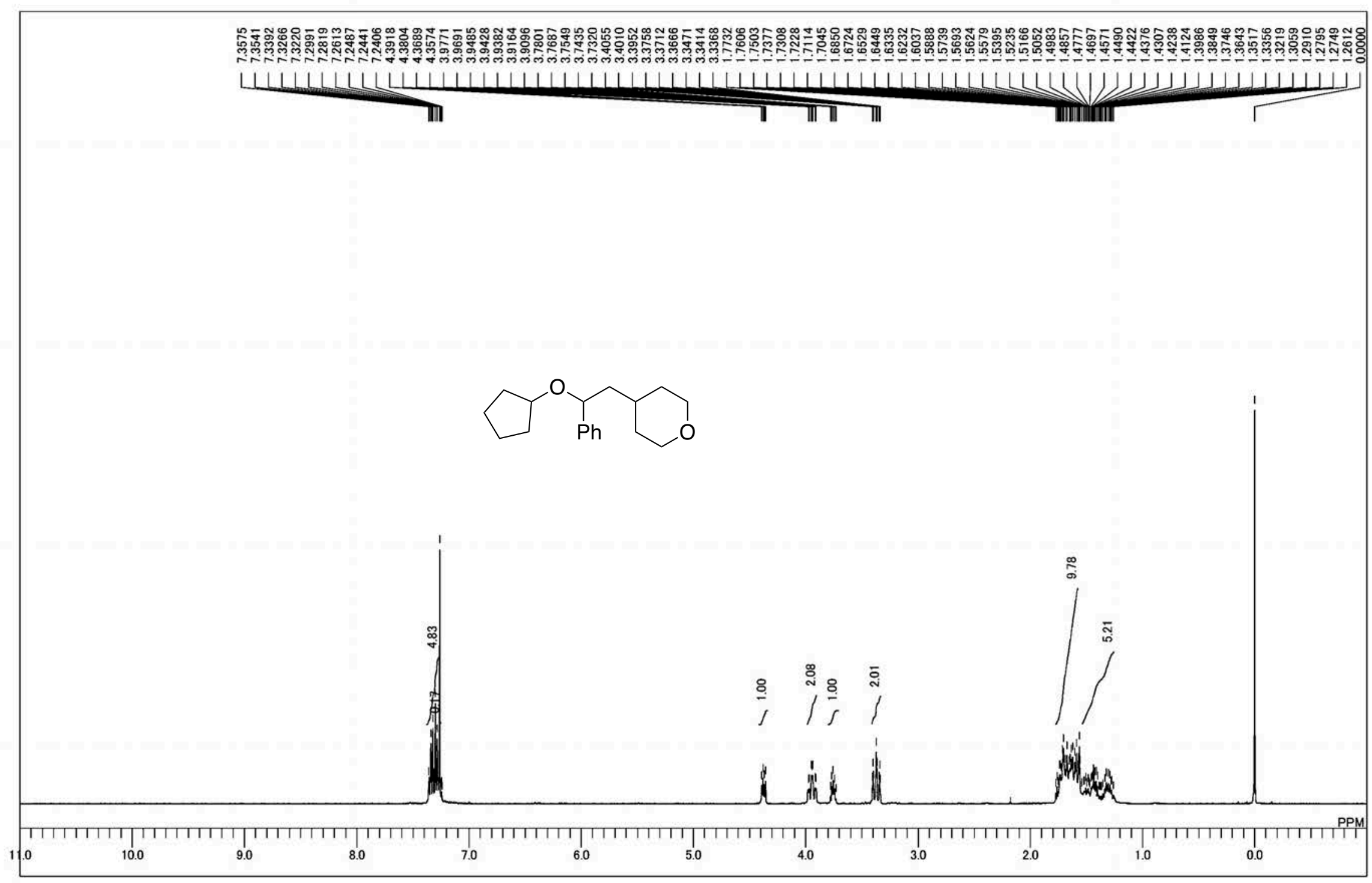

${ }^{1} \mathrm{H}$ NMR spectrum of $\mathbf{4 a a k}\left(400 \mathrm{MHz}, \mathrm{CDCl}_{3}\right)$ 


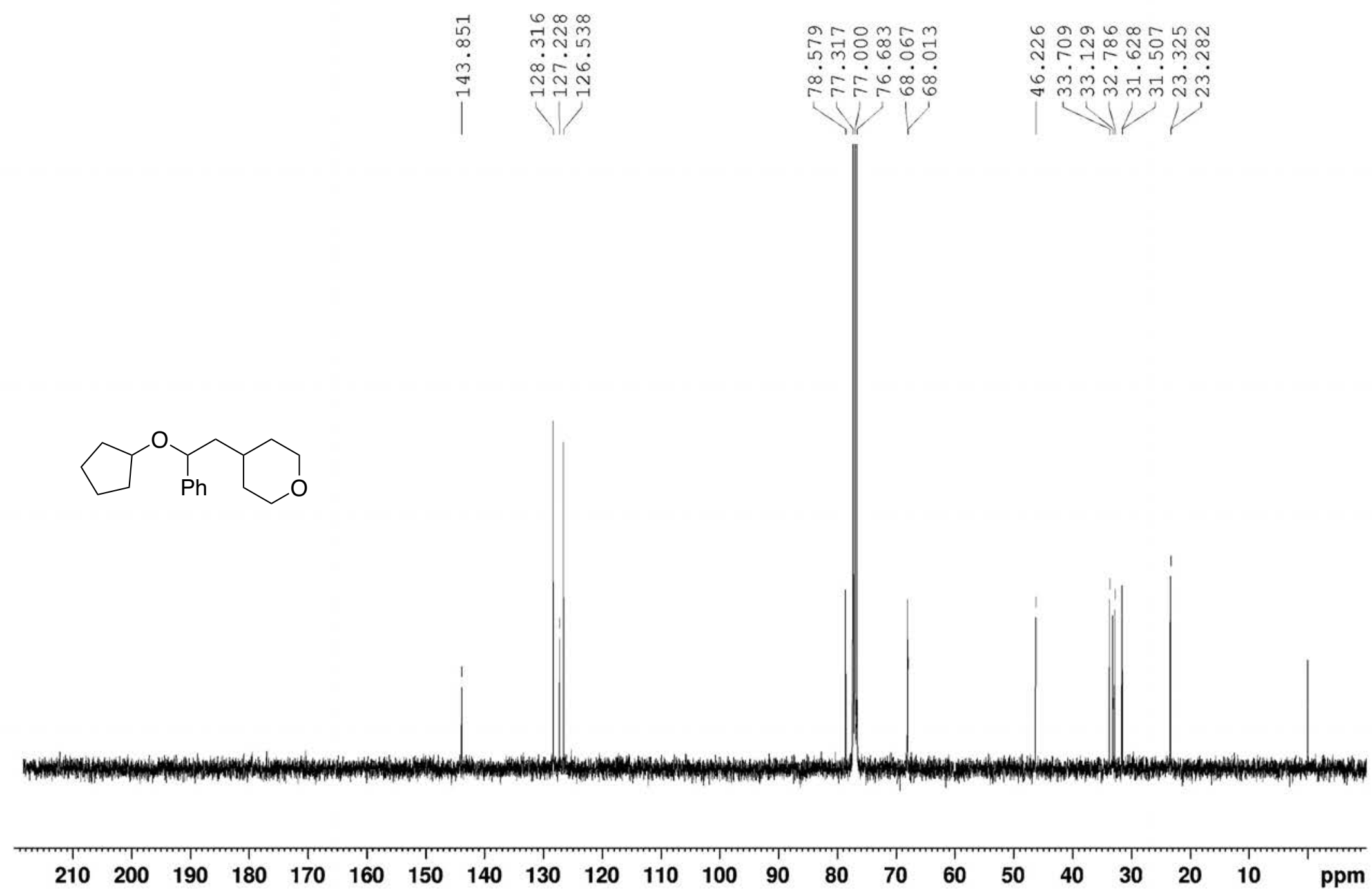

${ }^{13} \mathrm{C}$ NMR spectrum of $\mathbf{4 a a k}\left(100.6 \mathrm{MHz}, \mathrm{CDCl}_{3}\right)$ 


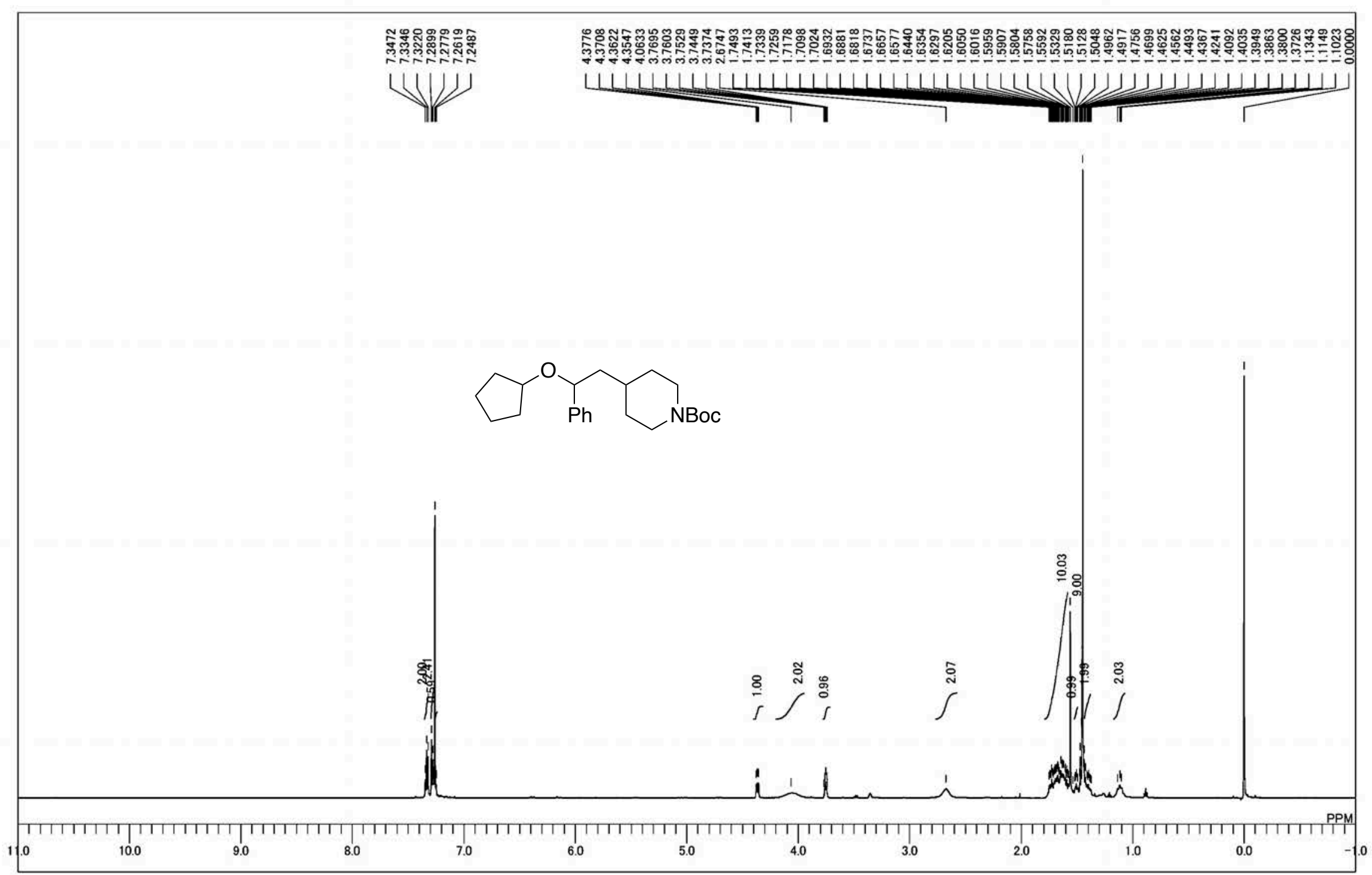

${ }^{1} \mathrm{H}$ NMR spectrum of 4 aal $\left(400 \mathrm{MHz}, \mathrm{CDCl}_{3}\right)$ 


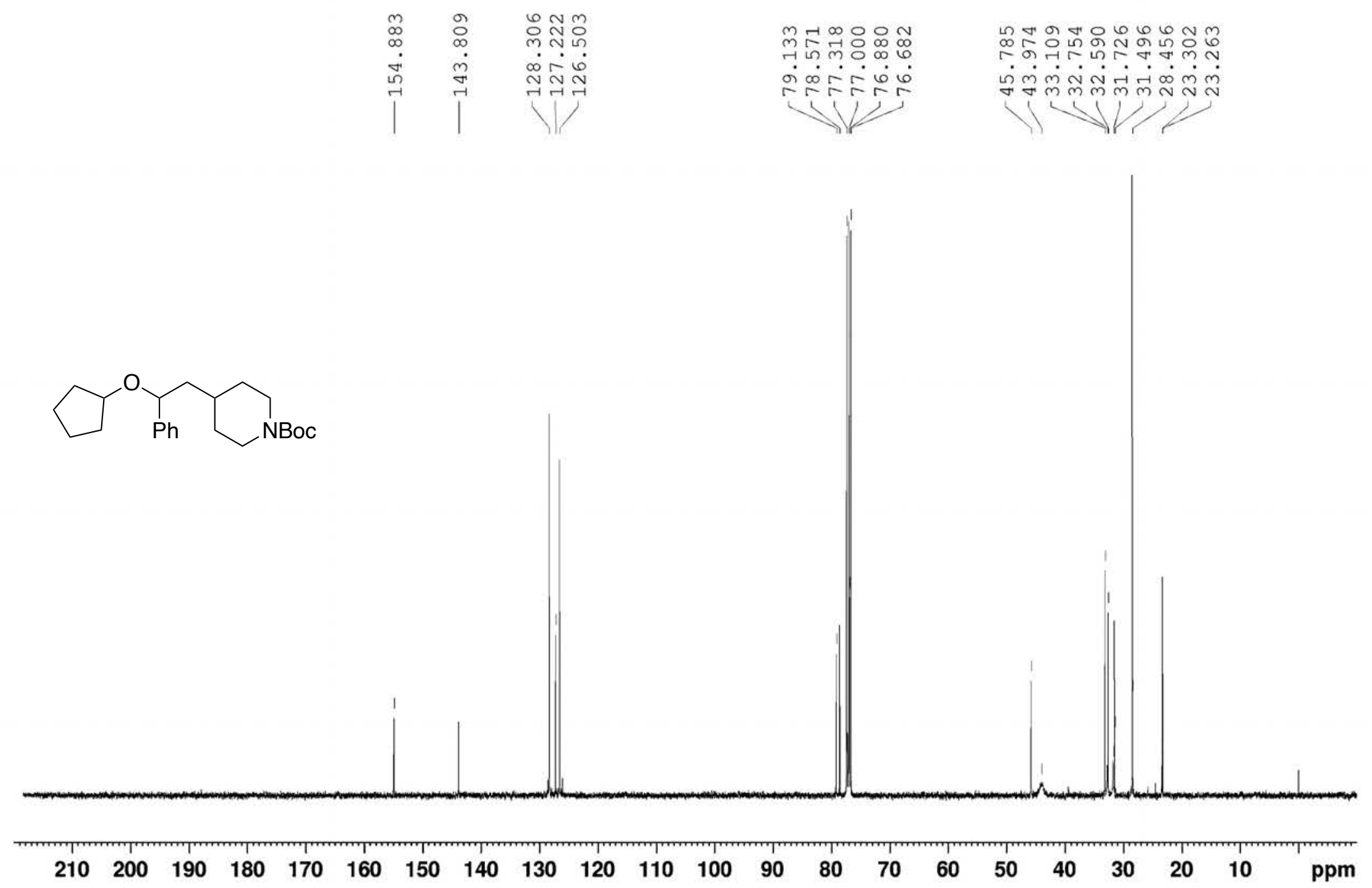

${ }^{13} \mathrm{C}$ NMR spectrum of 4 aal $\left(100.6 \mathrm{MHz}, \mathrm{CDCl}_{3}\right)$ 


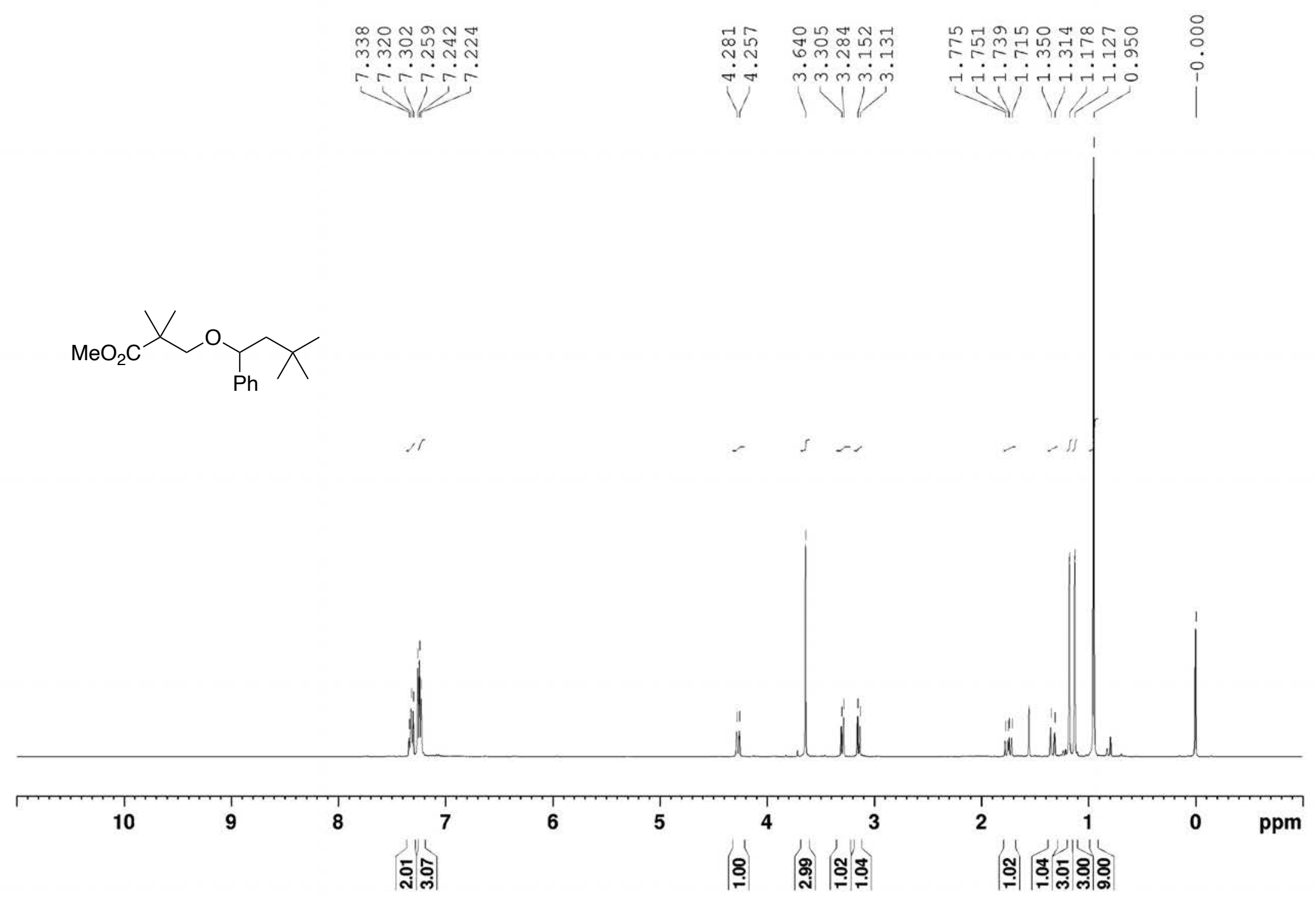

${ }^{1} \mathrm{H}$ NMR spectrum of $\mathbf{4 b a a}\left(400 \mathrm{MHz}, \mathrm{CDCl}_{3}\right.$ ) 


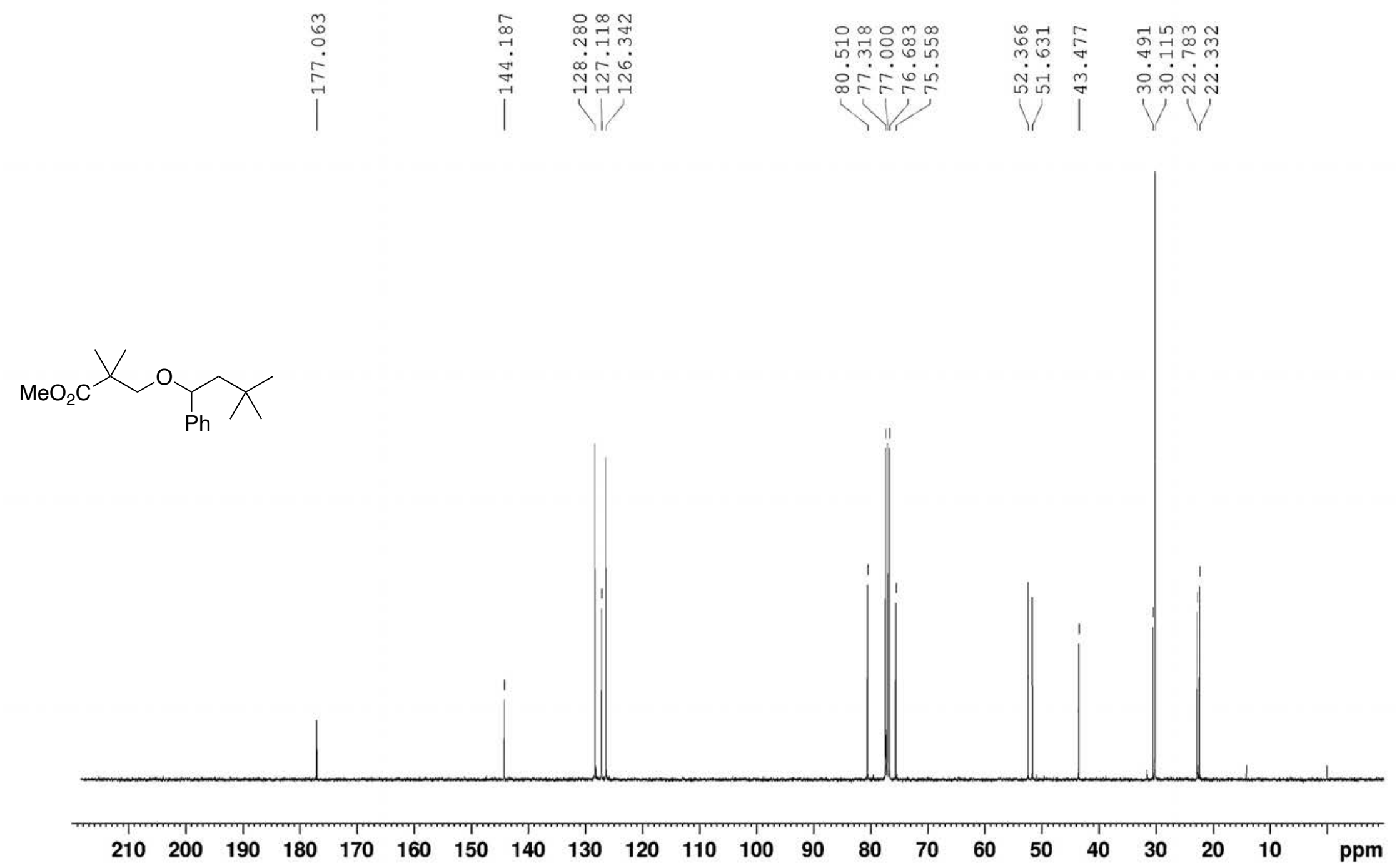

${ }^{13} \mathrm{C}$ NMR spectrum of $\mathbf{4 b a a}\left(100.6 \mathrm{MHz}, \mathrm{CDCl}_{3}\right)$ 


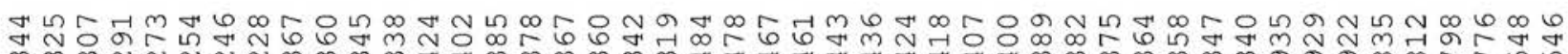
$-\rightarrow$ mंm
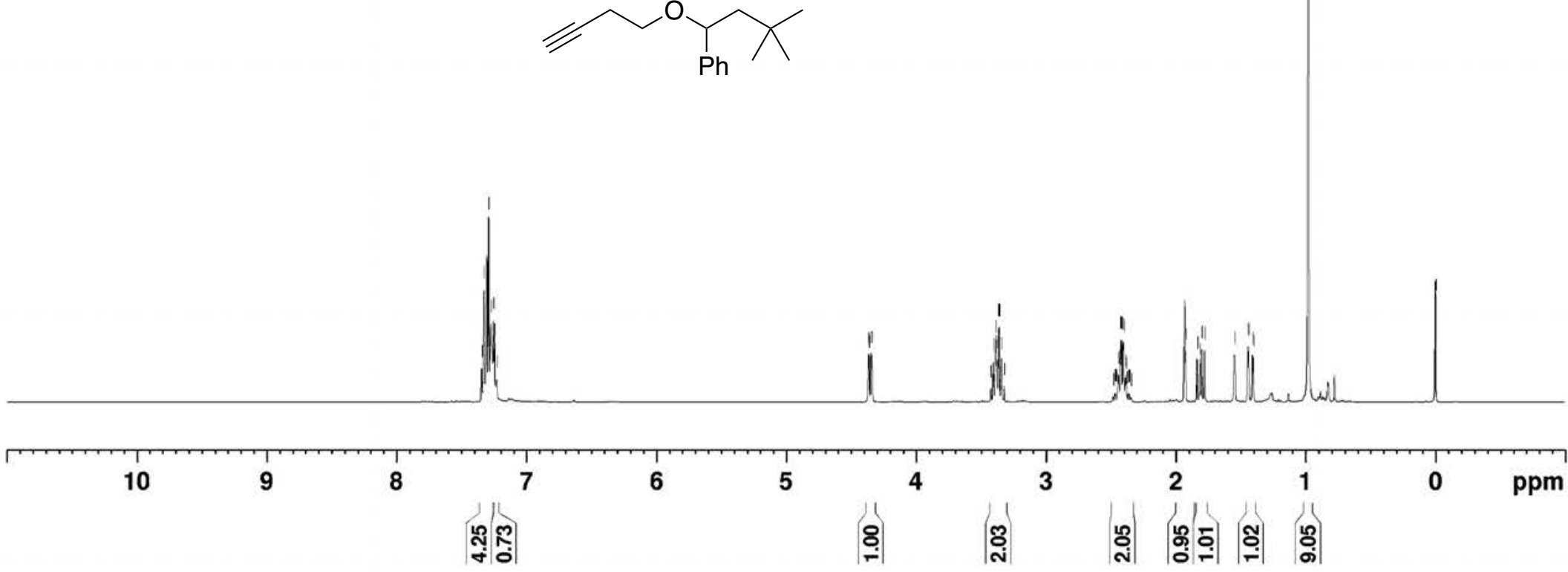

${ }^{1} \mathrm{H}$ NMR spectrum of 4 caa $\left(400 \mathrm{MHz}, \mathrm{CDCl}_{3}\right)$ 


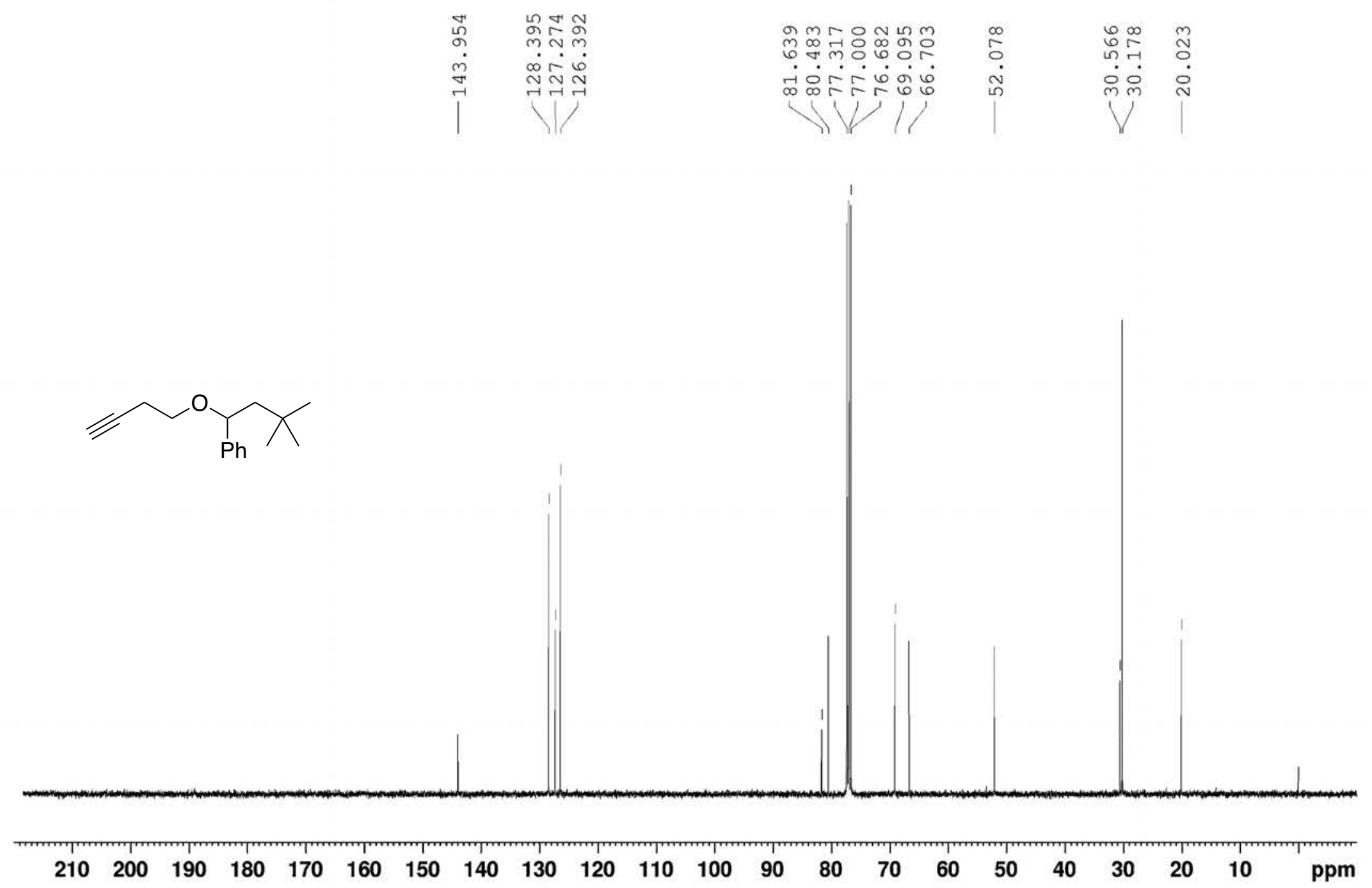

${ }^{13} \mathrm{C}$ NMR spectrum of $\mathbf{4 c a a}\left(100.6 \mathrm{MHz}, \mathrm{CDCl}_{3}\right)$ 


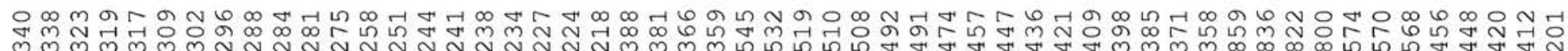
.

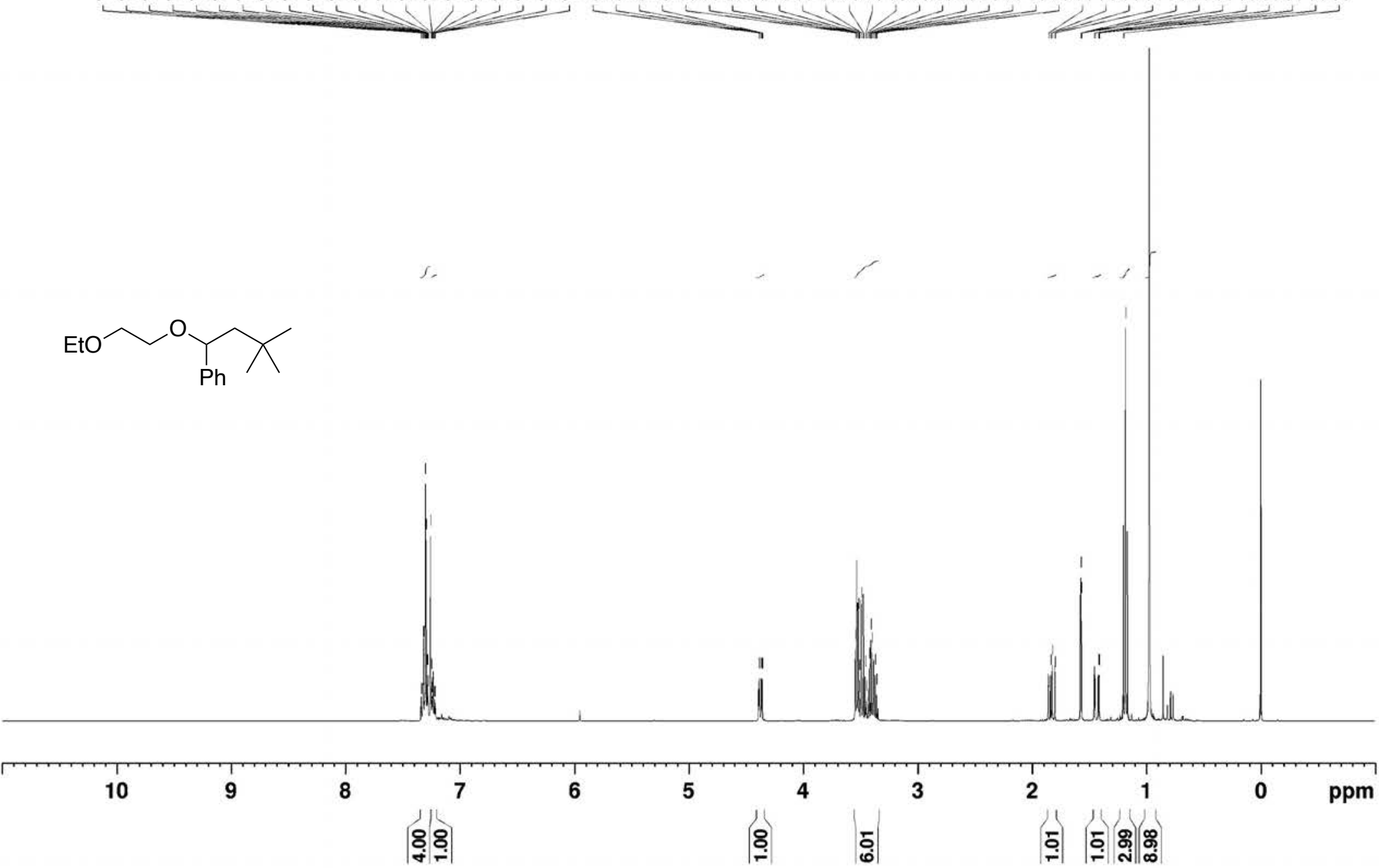

${ }^{1} \mathrm{H}$ NMR spectrum of 4 daa $\left(400 \mathrm{MHz}, \mathrm{CDCl}_{3}\right)$ 


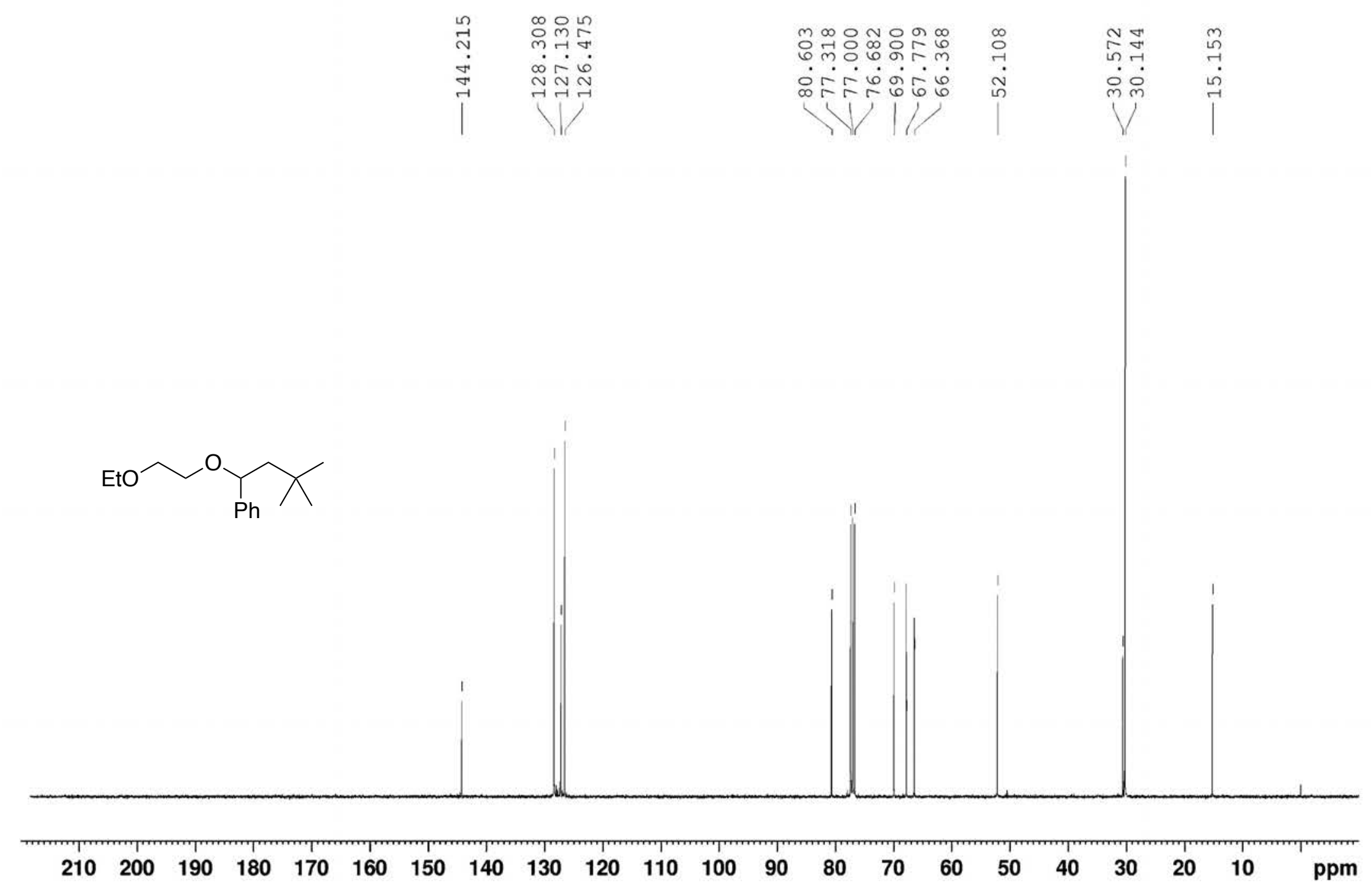

${ }^{13} \mathrm{C}$ NMR spectrum of 4 daa $\left(100.6 \mathrm{MHz}, \mathrm{CDCl}_{3}\right)$ 


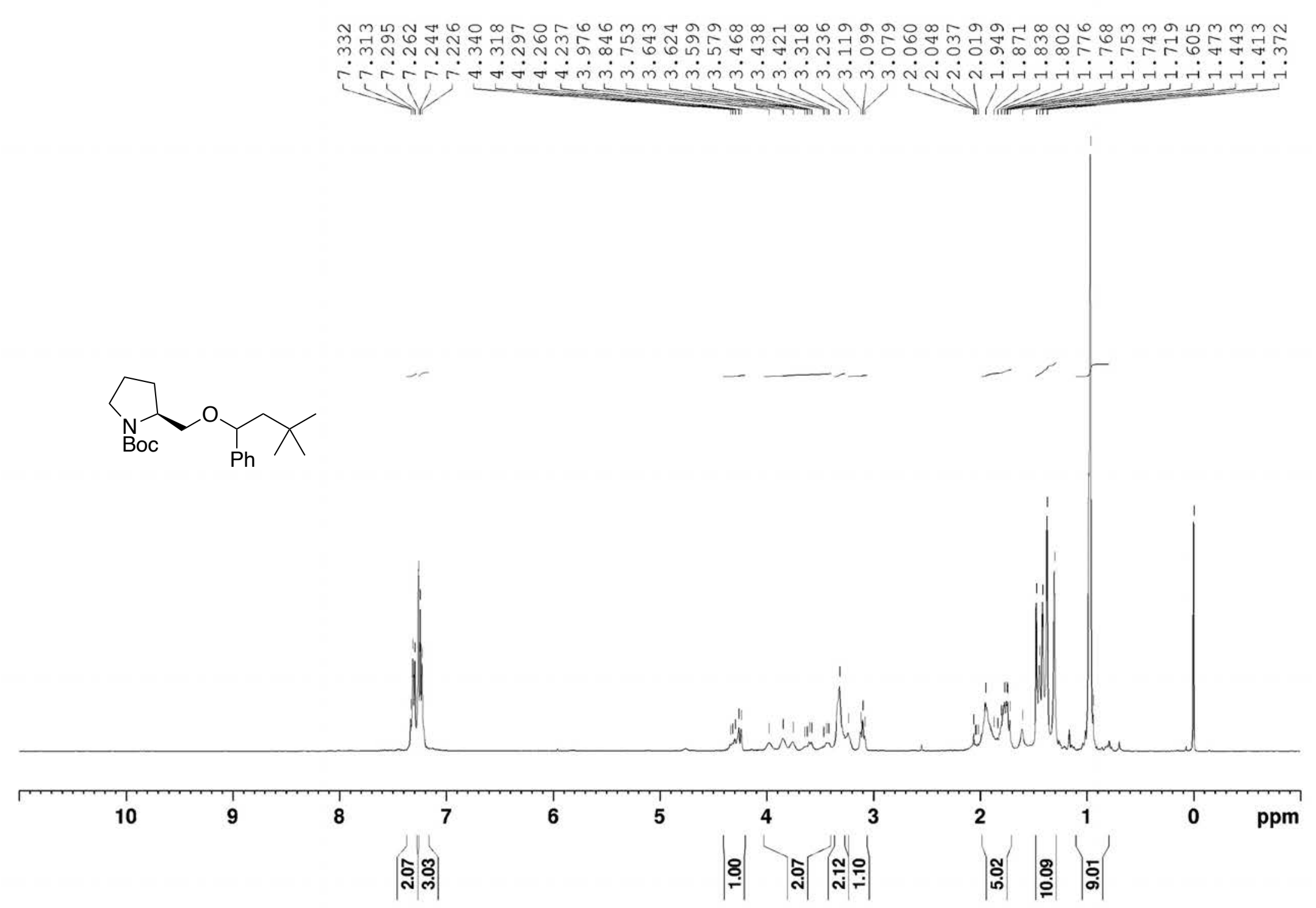

${ }^{1} \mathrm{H}$ NMR spectrum of $4 \mathbf{e a a}\left(400 \mathrm{MHz}, \mathrm{CDCl}_{3}\right)$ 


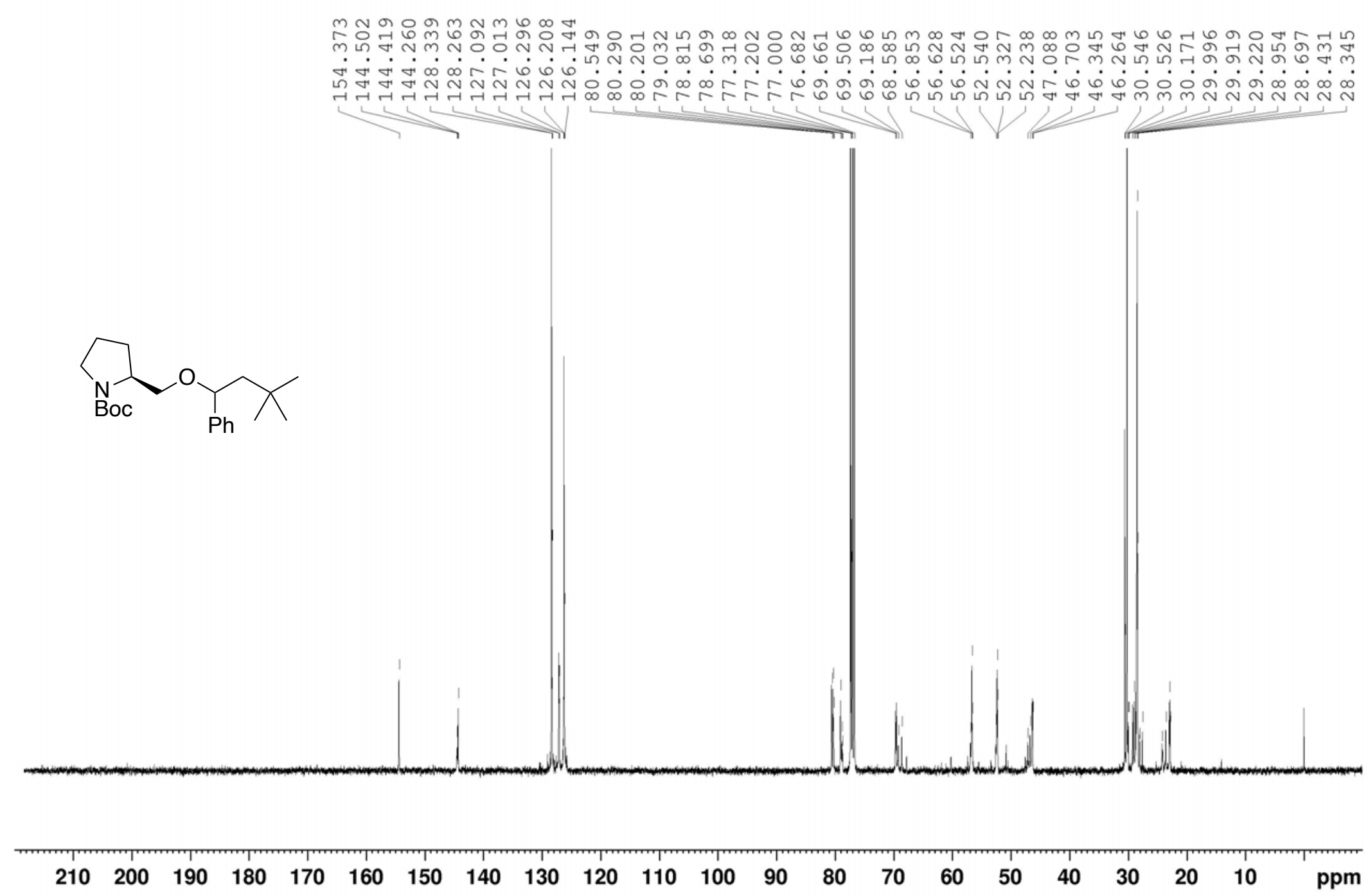

${ }^{13} \mathrm{C}$ NMR spectrum of 4 eaa $\left(100.6 \mathrm{MHz}, \mathrm{CDCl}_{3}\right)$ 


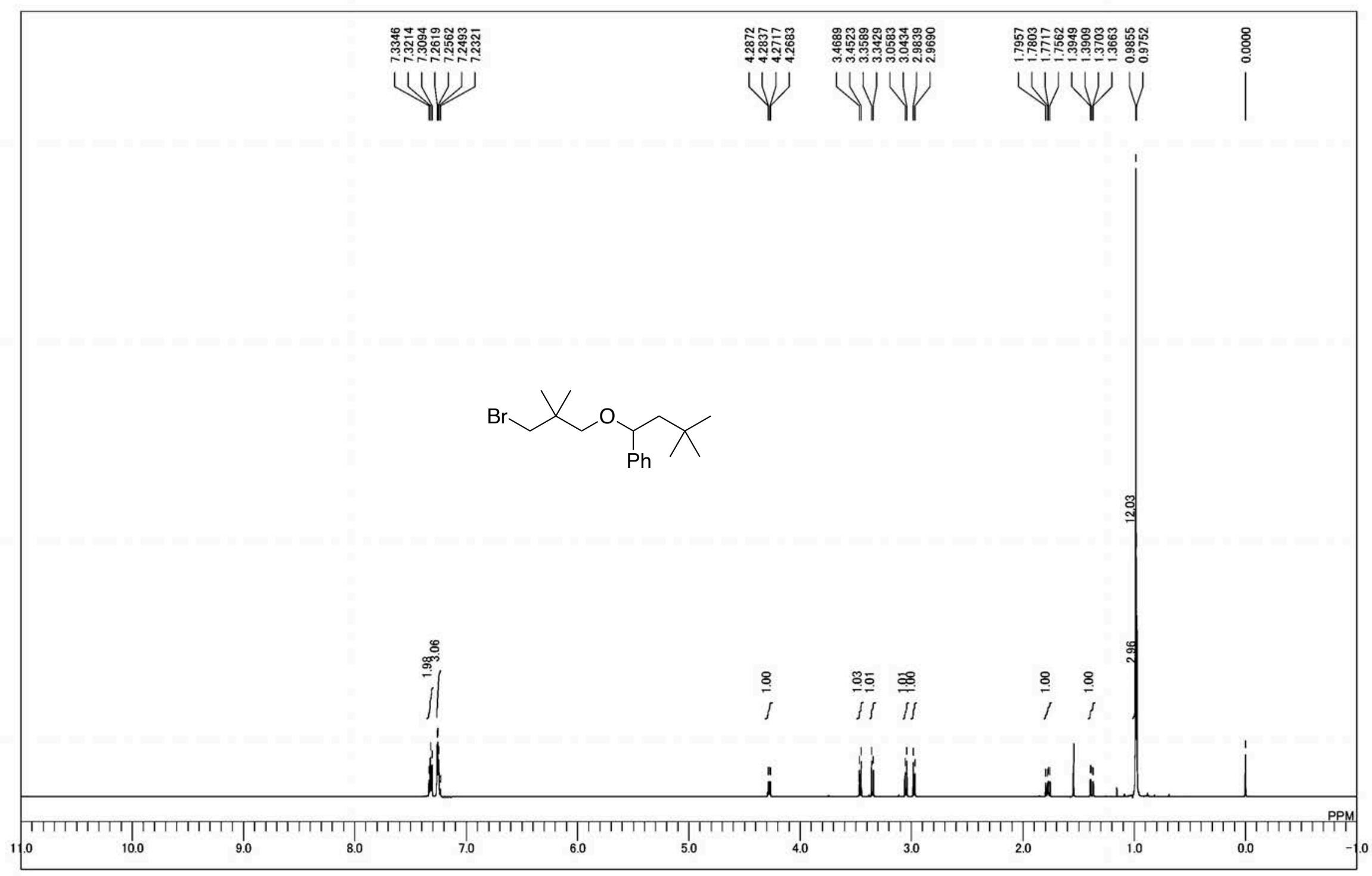

${ }^{1} \mathrm{H}$ NMR spectrum of 4 faa $\left(600 \mathrm{MHz}, \mathrm{CDCl}_{3}\right)$ 

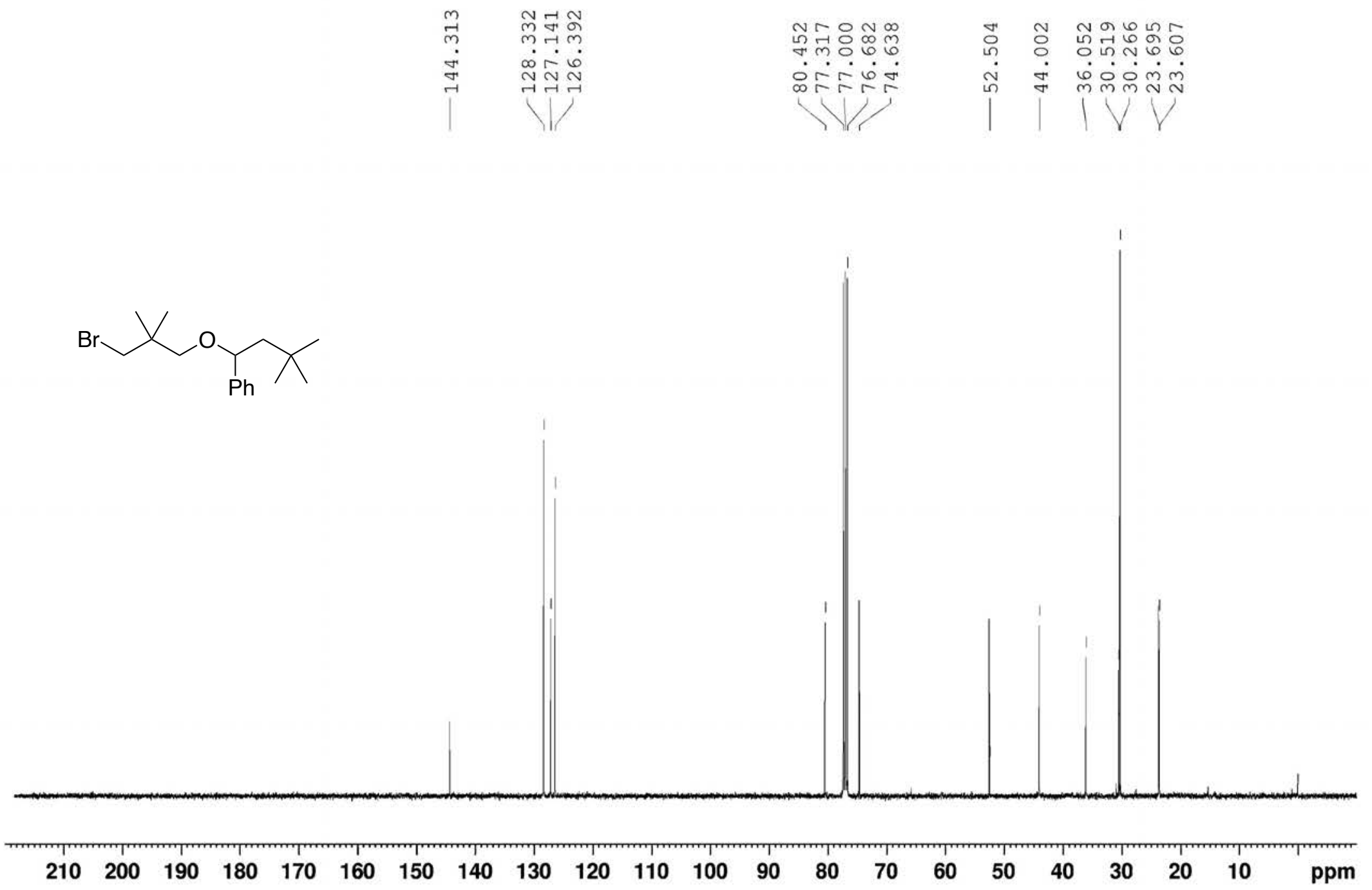

${ }^{13} \mathrm{C}$ NMR spectrum of $\mathbf{4 f a a}\left(100.6 \mathrm{MHz}, \mathrm{CDCl}_{3}\right)$ 


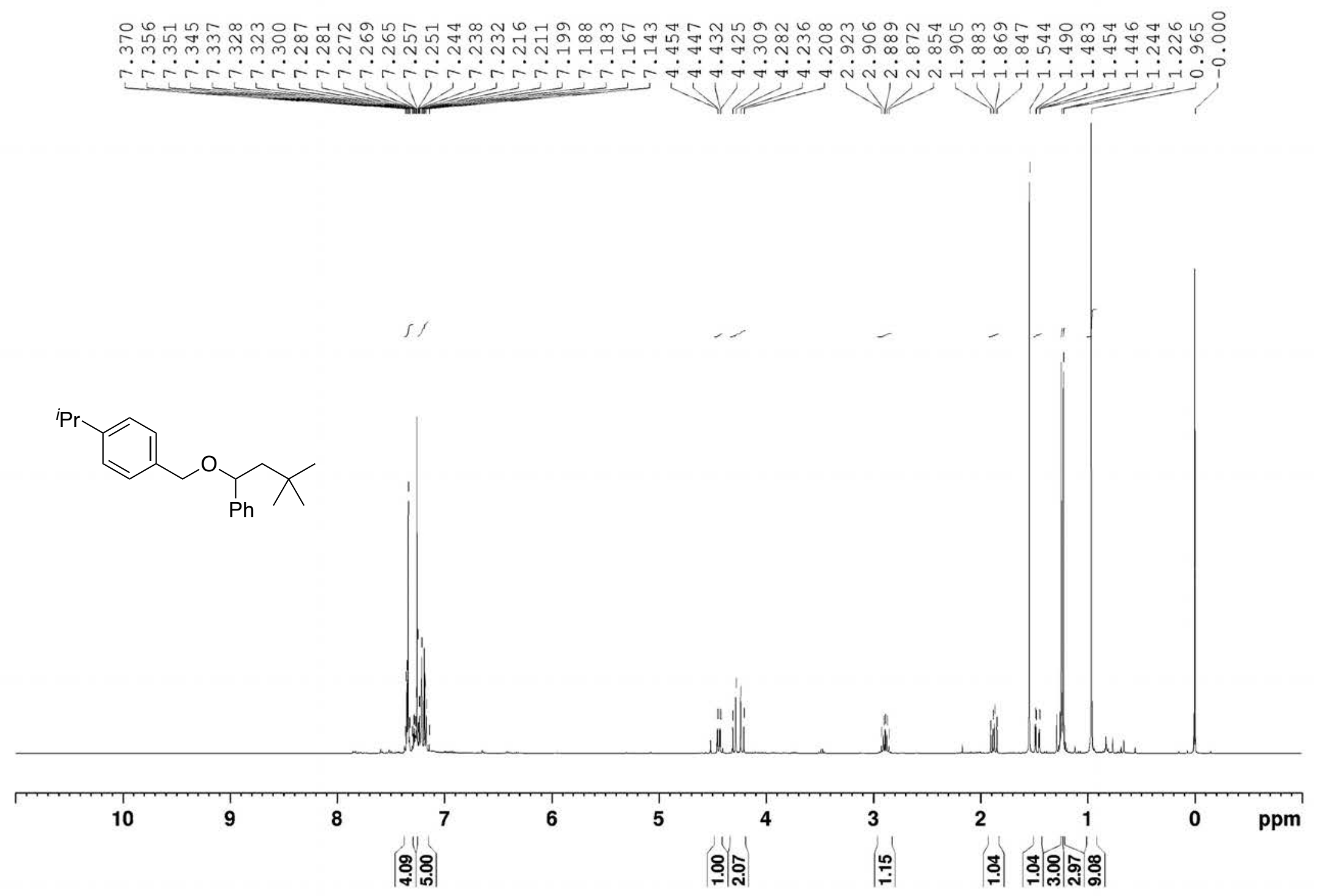

${ }^{1} \mathrm{H}$ NMR spectrum of 4 gaa $\left(400 \mathrm{MHz}, \mathrm{CDCl}_{3}\right.$ ) 


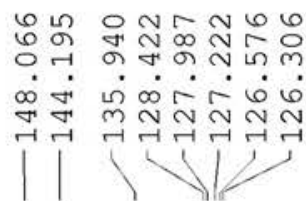

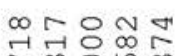

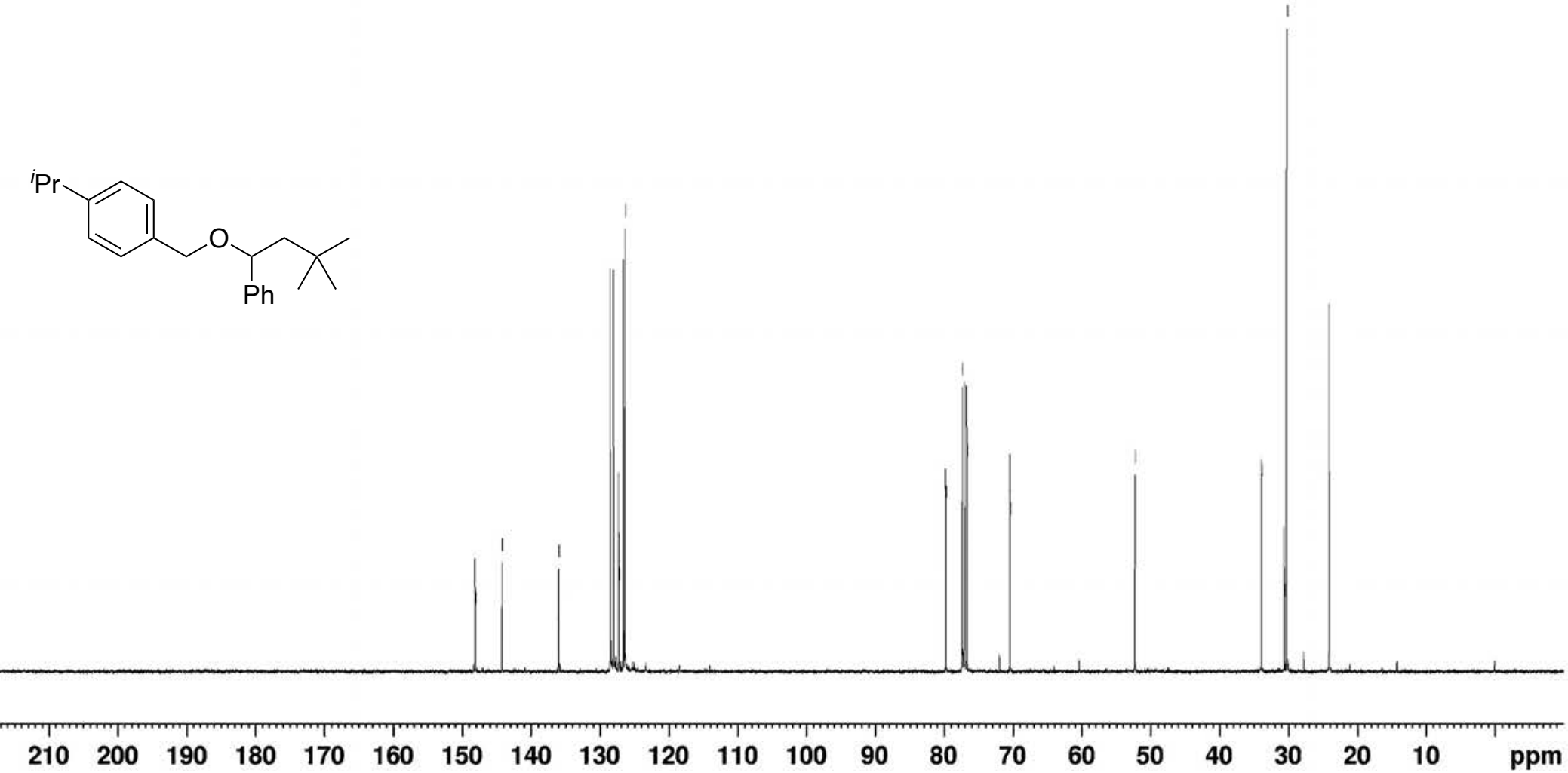

${ }^{13} \mathrm{C}$ NMR spectrum of 4 gaa $\left(100.6 \mathrm{MHz}, \mathrm{CDCl}_{3}\right)$ 


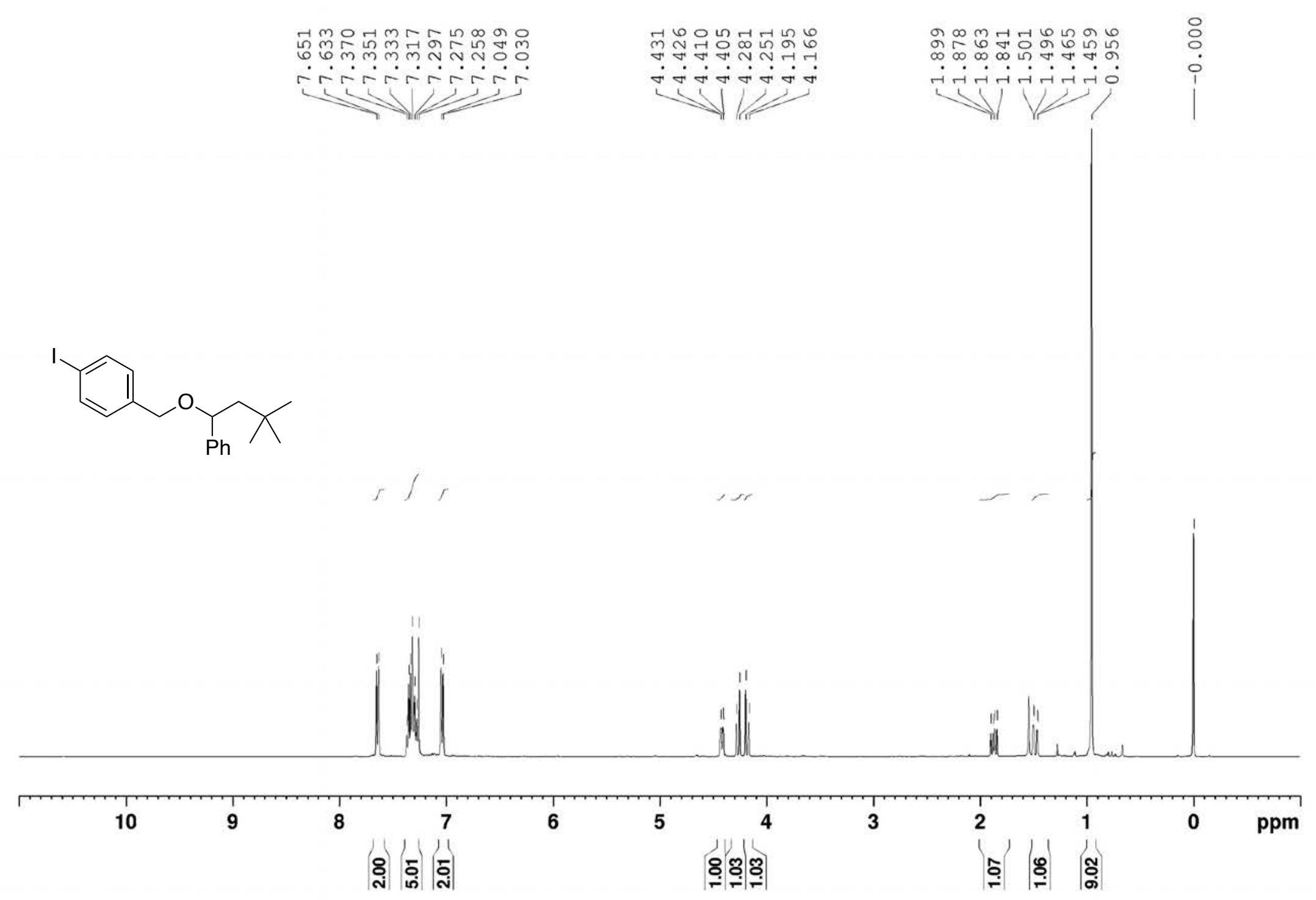

${ }^{1} \mathrm{H}$ NMR spectrum of 4 haa $\left(400 \mathrm{MHz}, \mathrm{CDCl}_{3}\right.$ ) 


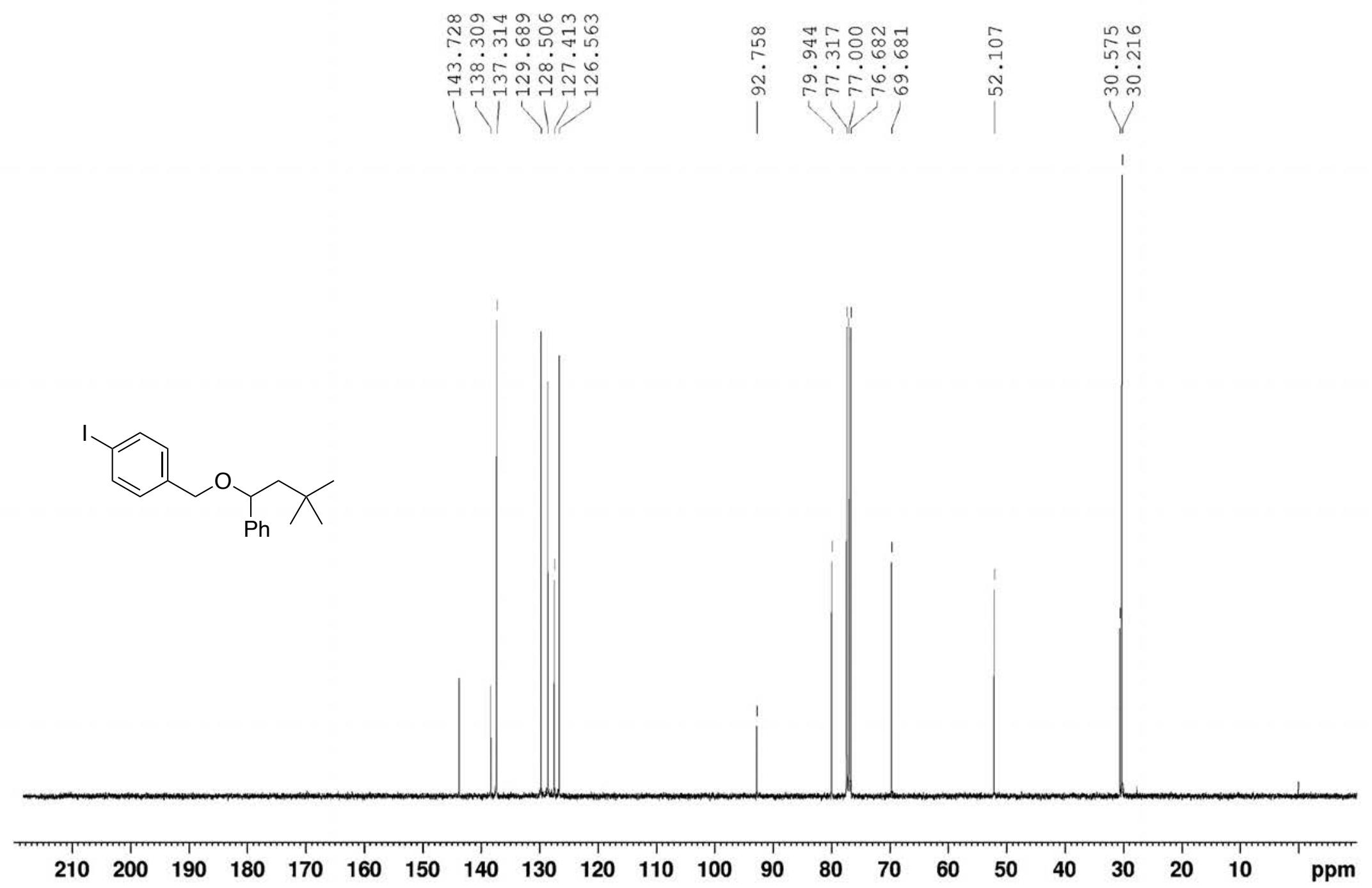

${ }^{13} \mathrm{C}$ NMR spectrum of 4 haa $\left(100.6 \mathrm{MHz}, \mathrm{CDCl}_{3}\right)$ 


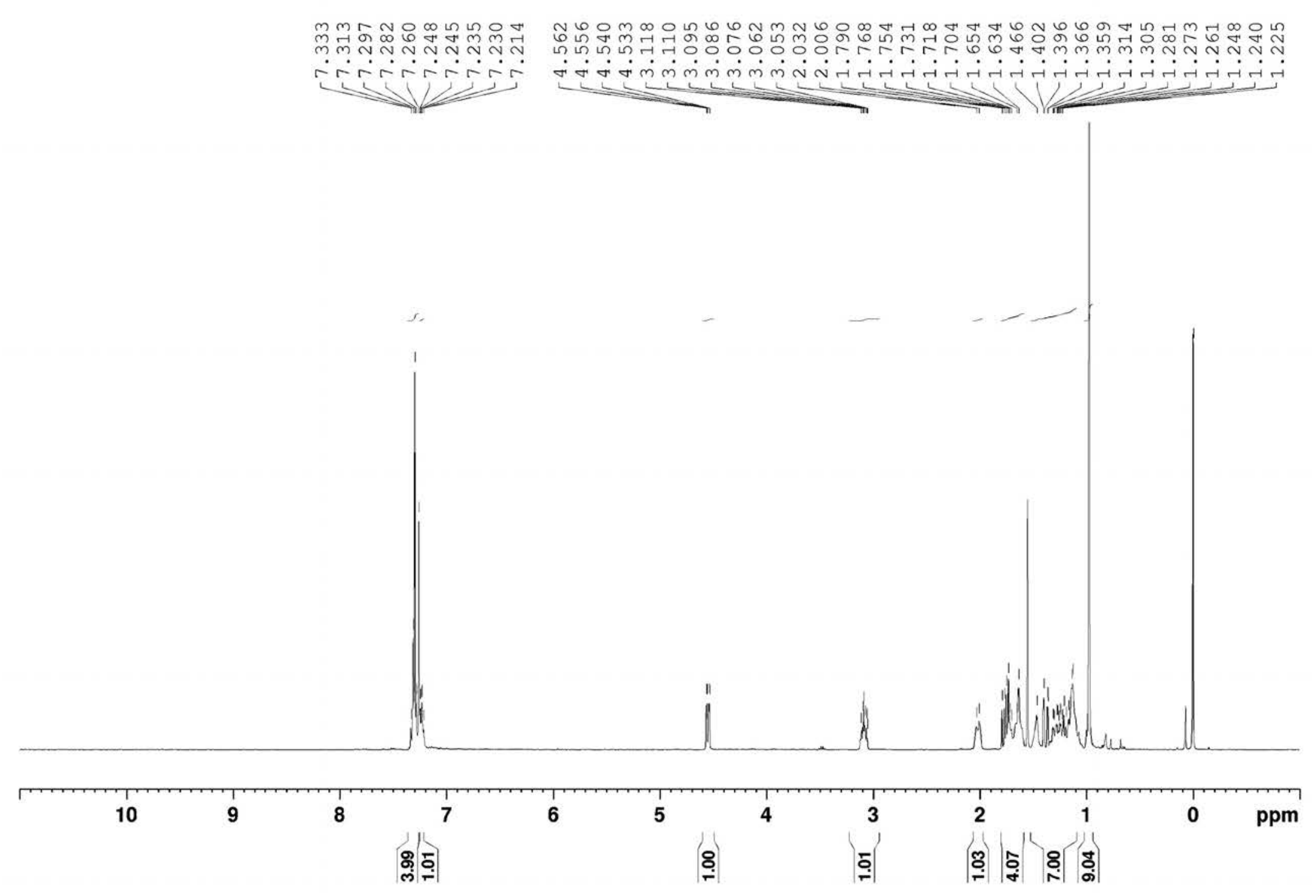

${ }^{1} \mathrm{H}$ NMR spectrum of 4 iaa $\left(400 \mathrm{MHz}, \mathrm{CDCl}_{3}\right)$ 


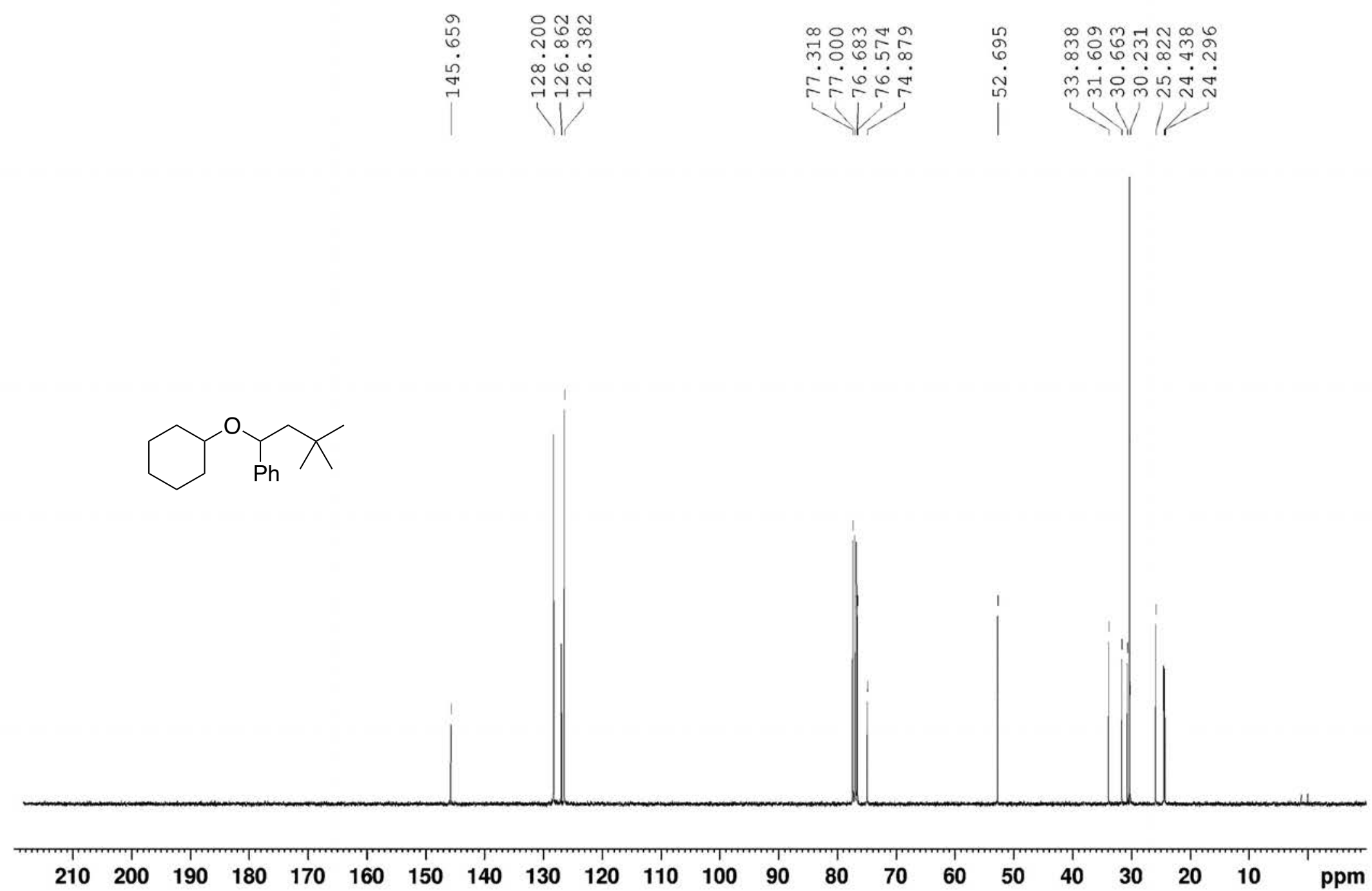

${ }^{13} \mathrm{C}$ NMR spectrum of 4 iaa $\left(100.6 \mathrm{MHz}, \mathrm{CDCl}_{3}\right)$ 


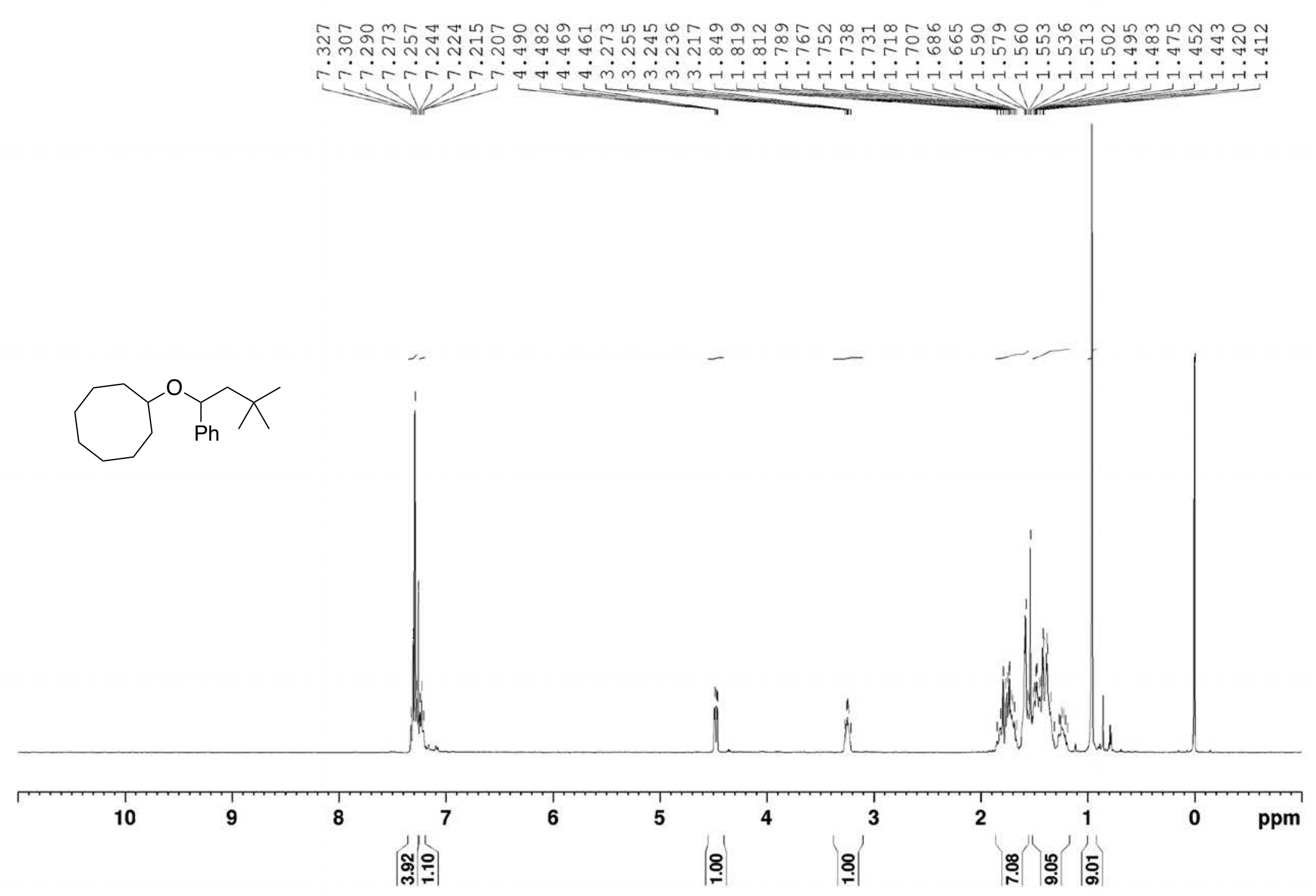

${ }^{1} \mathrm{H}$ NMR spectrum of $\mathbf{4 j a a}\left(400 \mathrm{MHz}, \mathrm{CDCl}_{3}\right)$ 


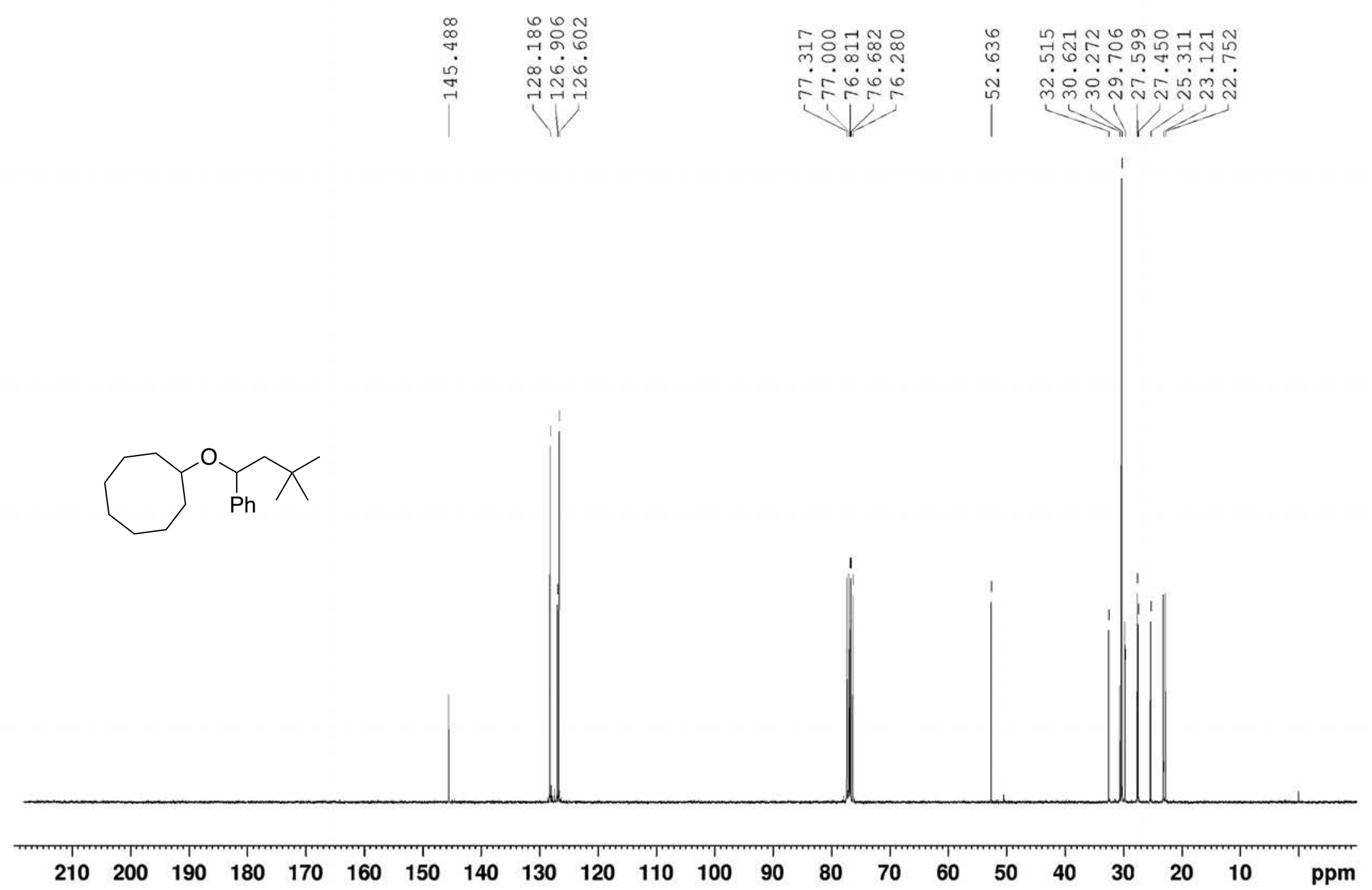

${ }^{13} \mathrm{C}$ NMR spectrum of $\mathbf{4 j a a}\left(100.6 \mathrm{MHz}, \mathrm{CDCl}_{3}\right)$ 
স্যু

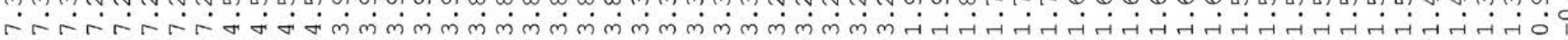

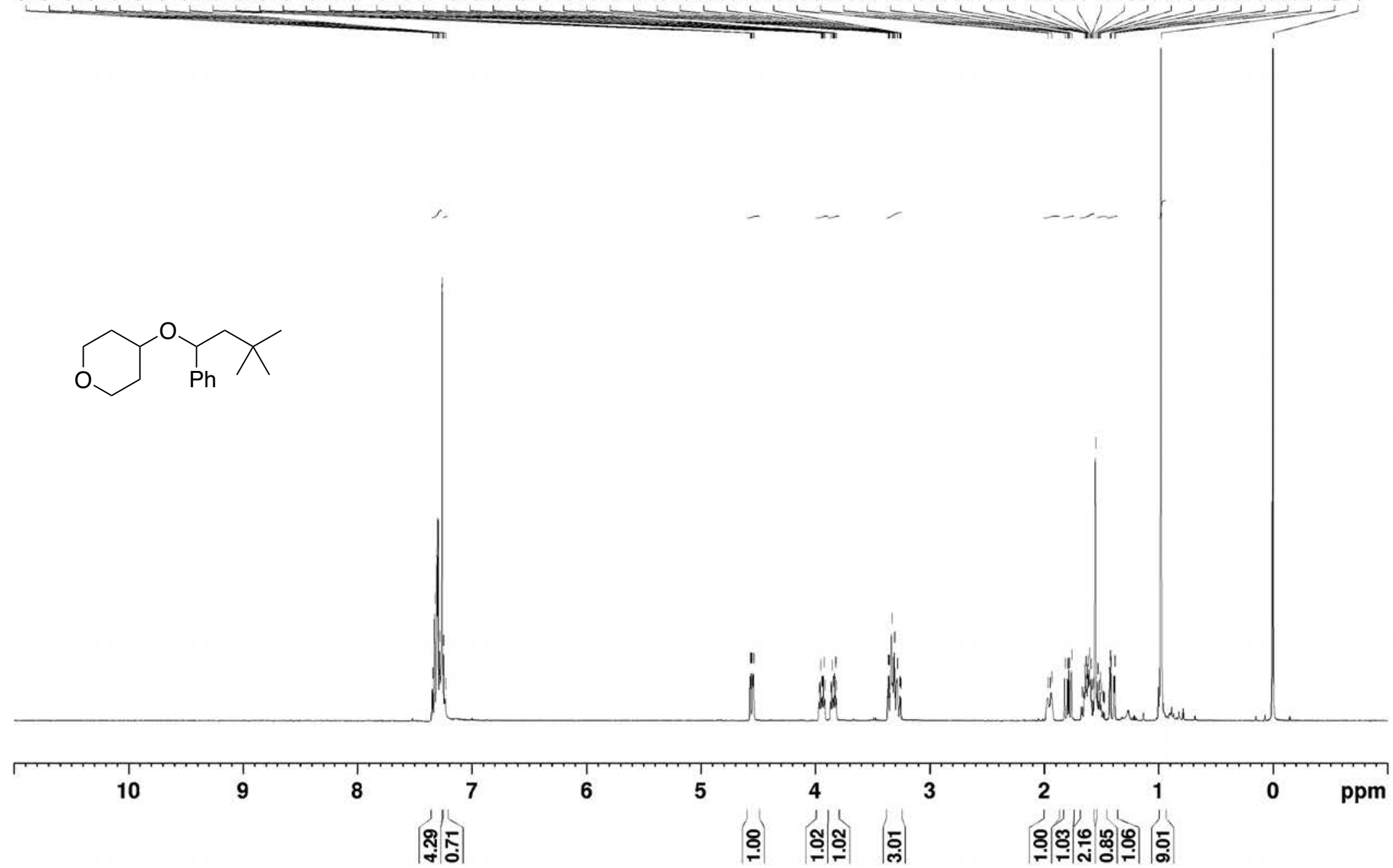

${ }^{1} \mathrm{H}$ NMR spectrum of $\mathbf{4} \mathbf{k a a}\left(400 \mathrm{MHz}, \mathrm{CDCl}_{3}\right)$ 


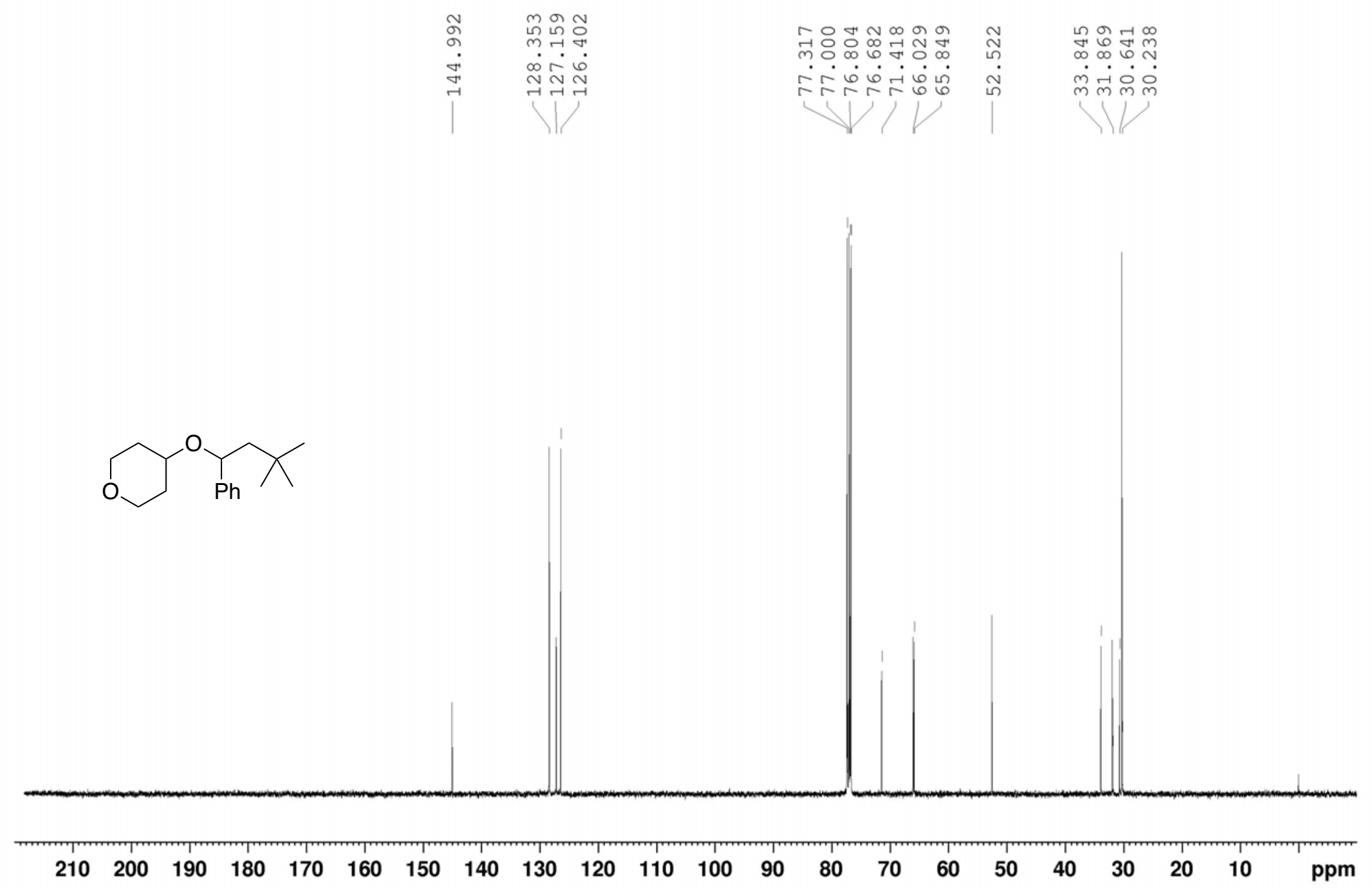

${ }^{13} \mathrm{C}$ NMR spectrum of $\mathbf{4} \mathbf{k a a}\left(100.6 \mathrm{MHz}, \mathrm{CDCl}_{3}\right)$ 


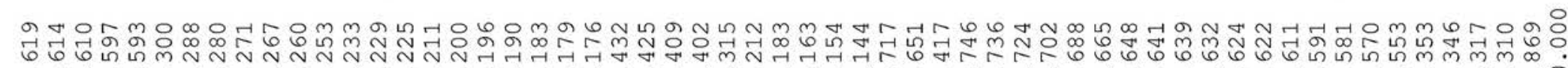
.

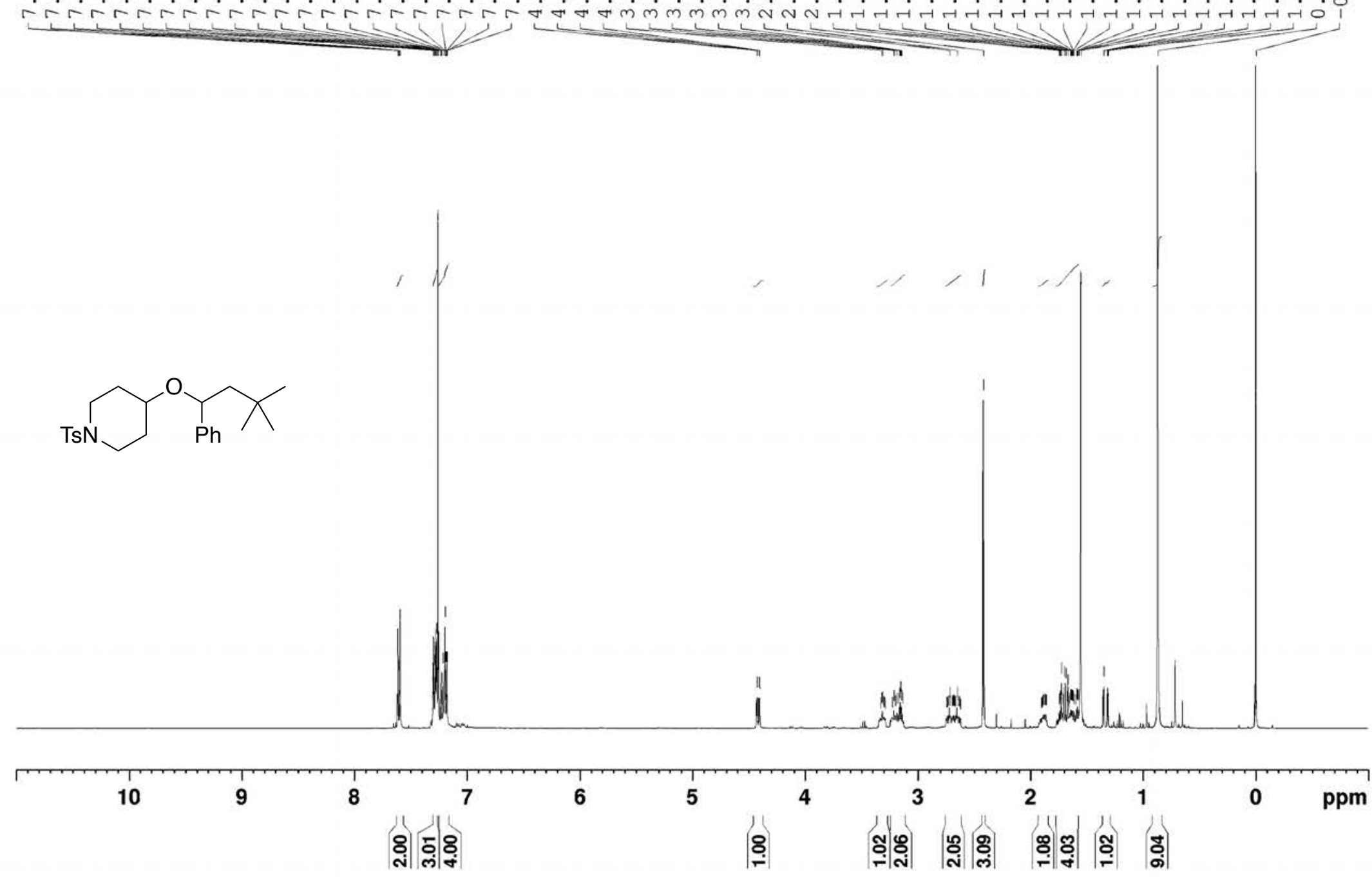

${ }^{1} \mathrm{H}$ NMR spectrum of 4laa (400 MHz, $\mathrm{CDCl}_{3}$ ) 


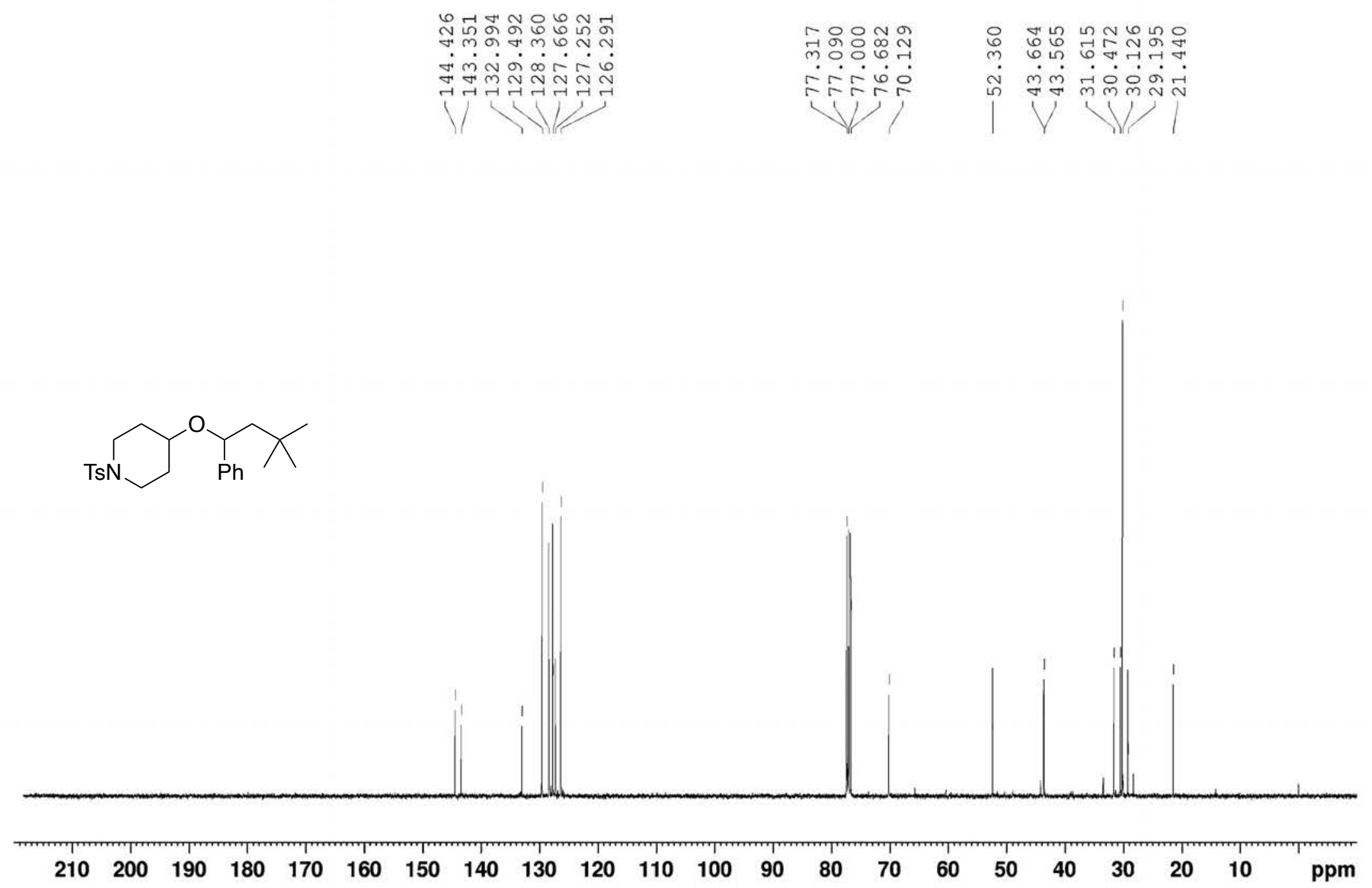

${ }^{13} \mathrm{C}$ NMR spectrum of 4 laa $\left(100.6 \mathrm{MHz}, \mathrm{CDCl}_{3}\right)$ 


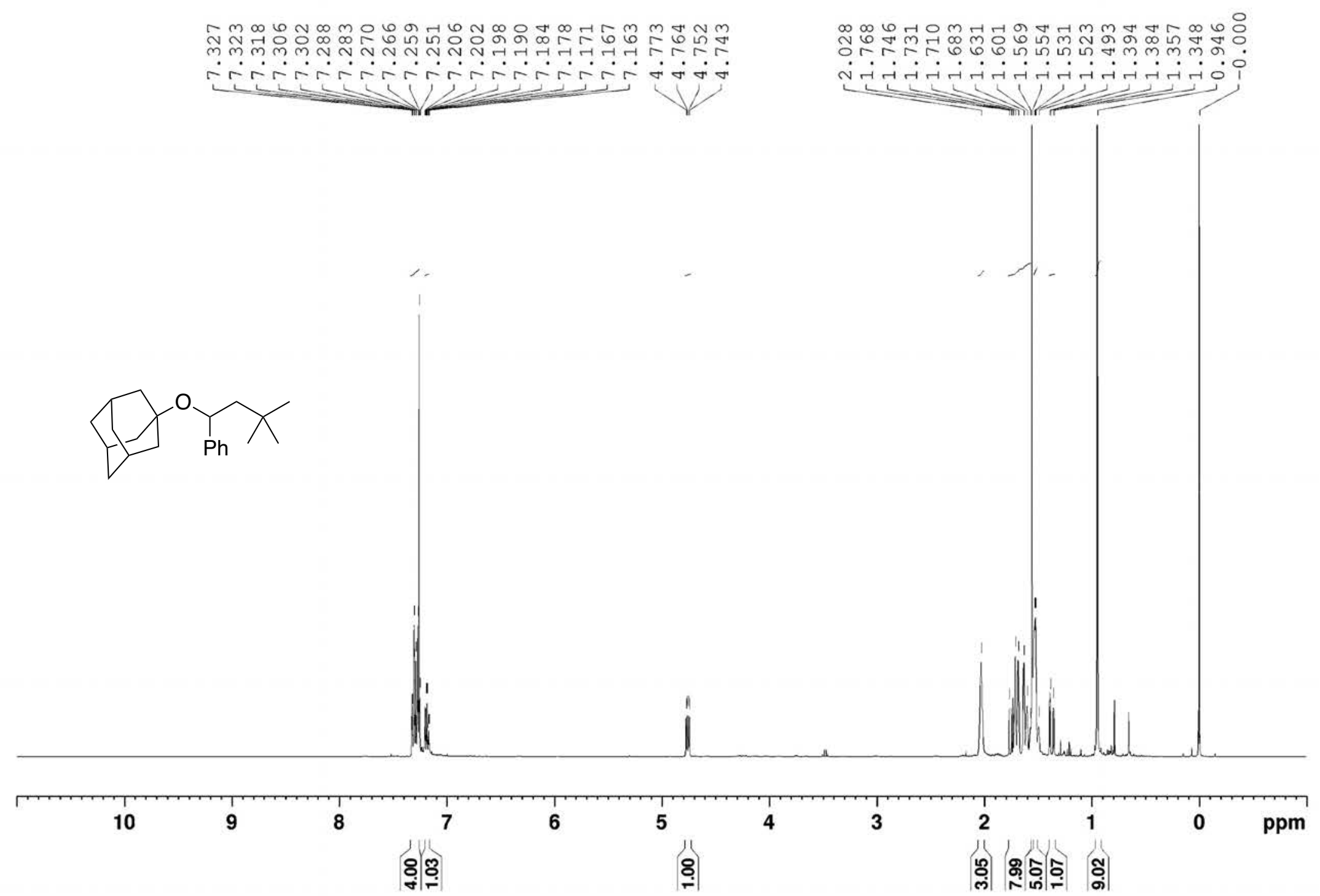

${ }^{1} \mathrm{H}$ NMR spectrum of $\mathbf{4 m a a}\left(400 \mathrm{MHz}, \mathrm{CDCl}_{3}\right)$ 


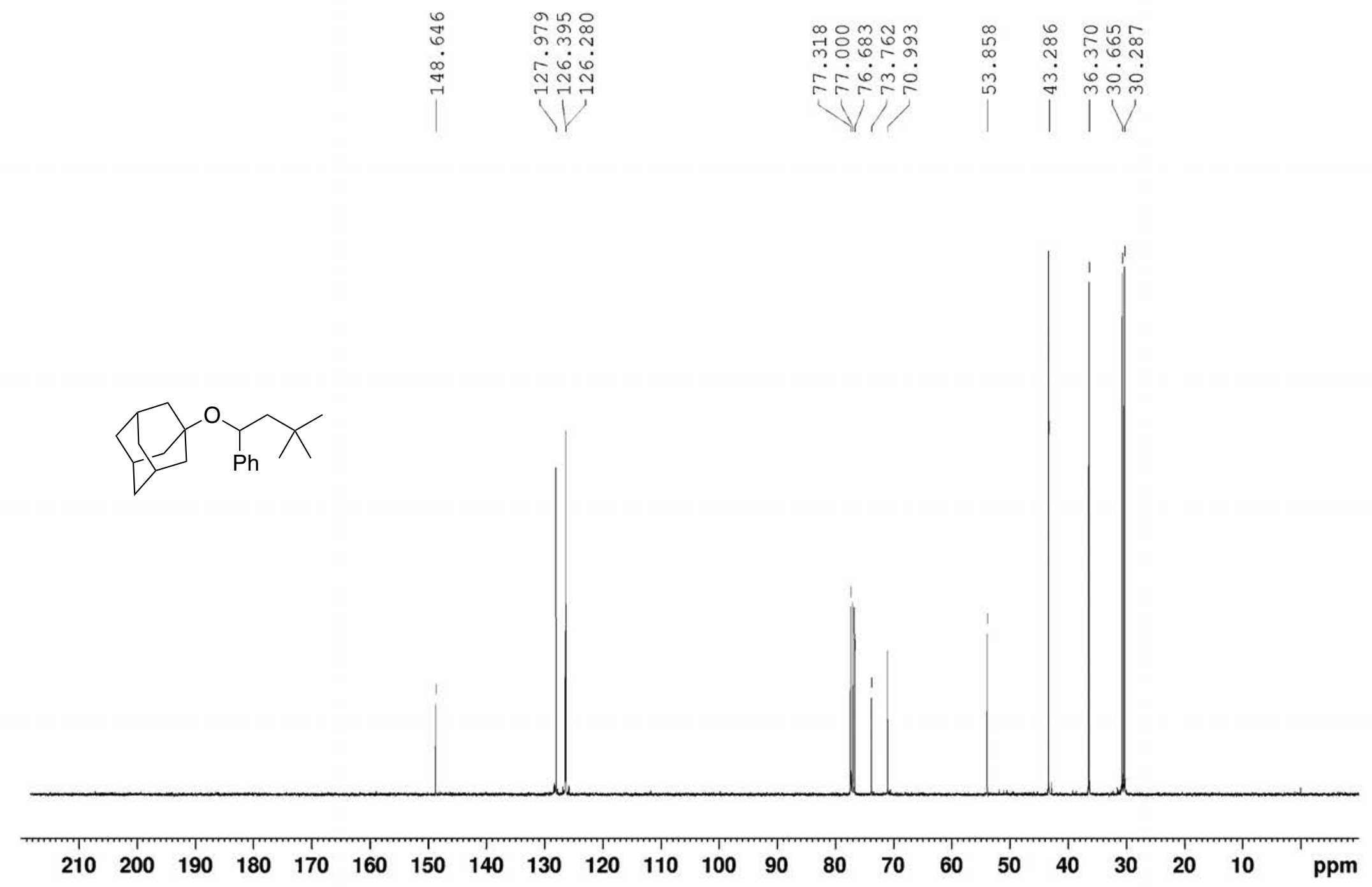

${ }^{13} \mathrm{C}$ NMR spectrum of 4 maa $\left(100.6 \mathrm{MHz}, \mathrm{CDCl}_{3}\right)$ 


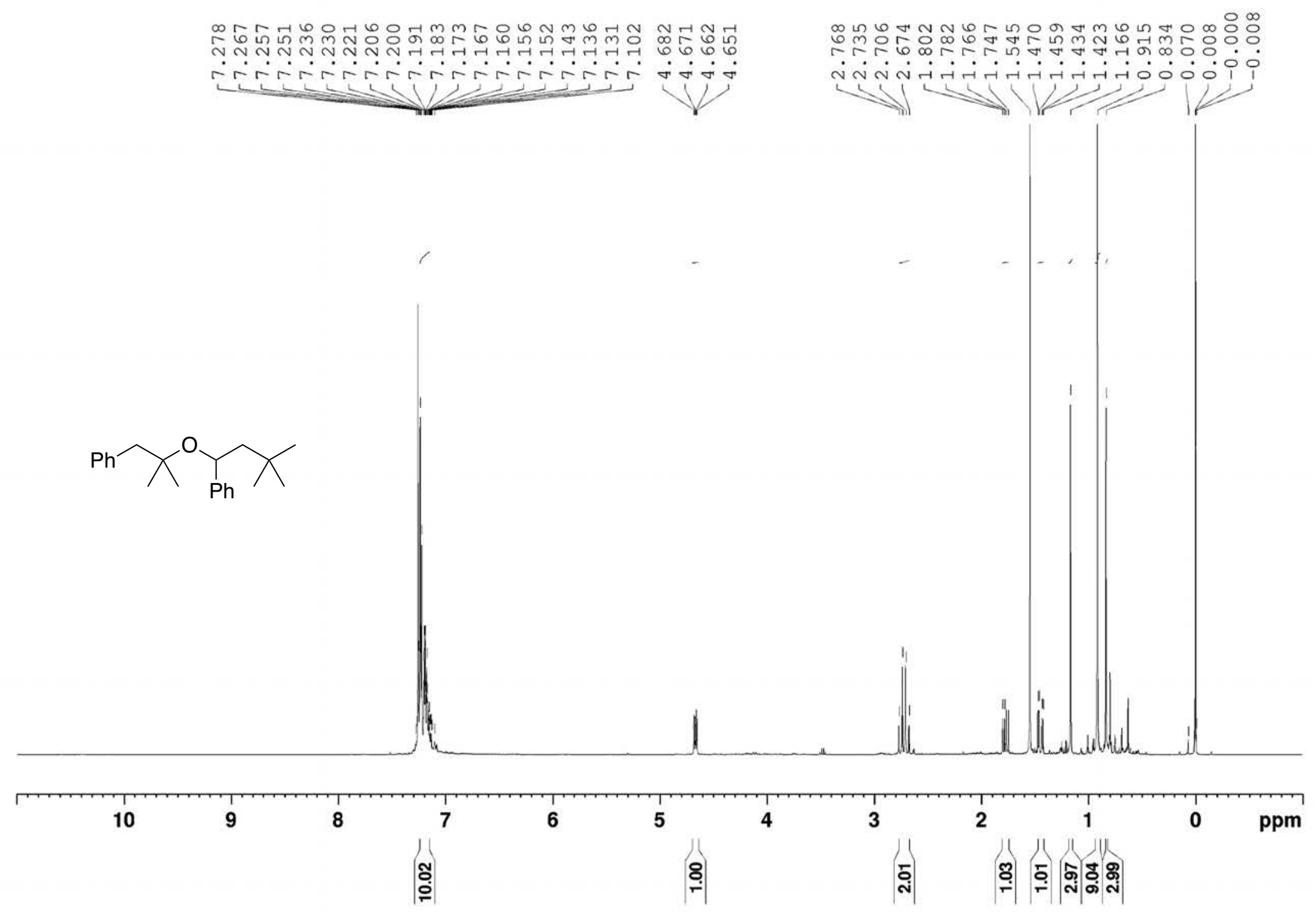

${ }^{1} \mathrm{H}$ NMR spectrum of 4 naa $\left(400 \mathrm{MHz}, \mathrm{CDCl}_{3}\right.$ ) 


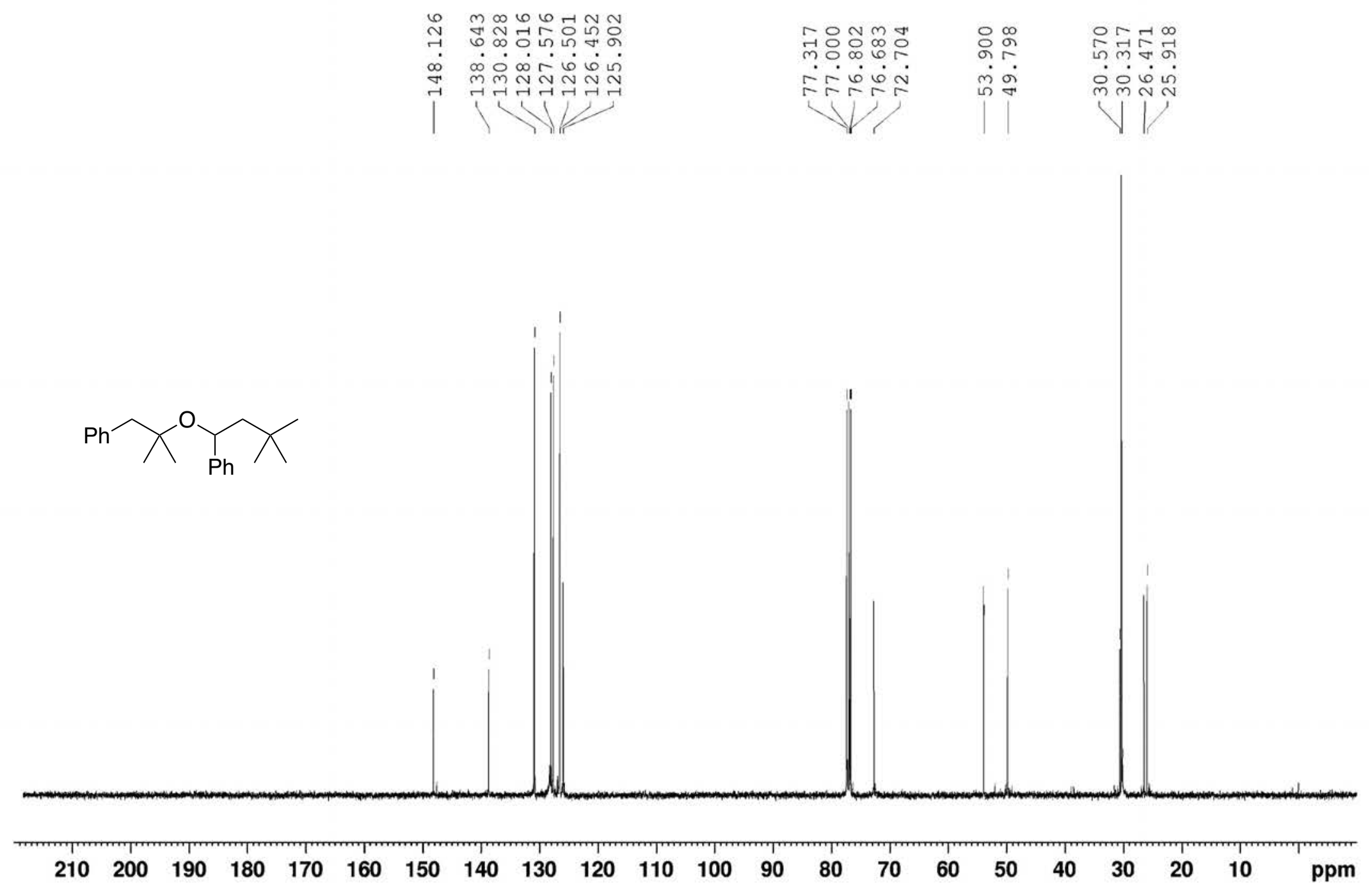

${ }^{13} \mathrm{C}$ NMR spectrum of 4 naa $\left(100.6 \mathrm{MHz}, \mathrm{CDCl}_{3}\right)$ 


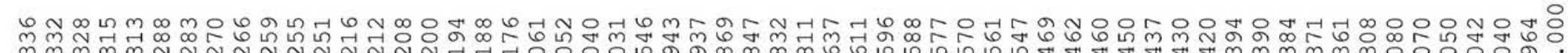

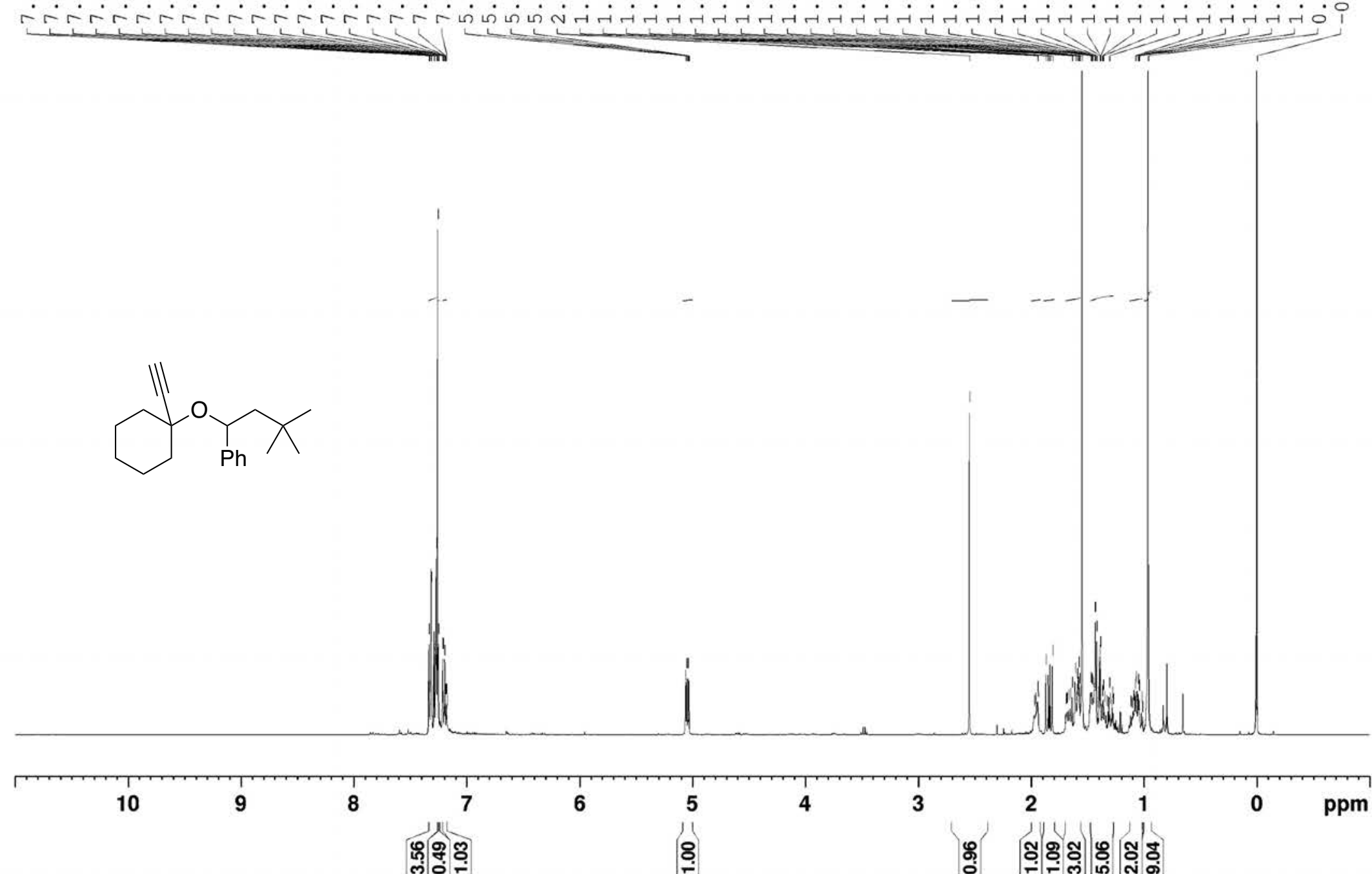

${ }^{1} \mathrm{H}$ NMR spectrum of $4 \mathbf{o a a}\left(400 \mathrm{MHz}, \mathrm{CDCl}_{3}\right)$ 


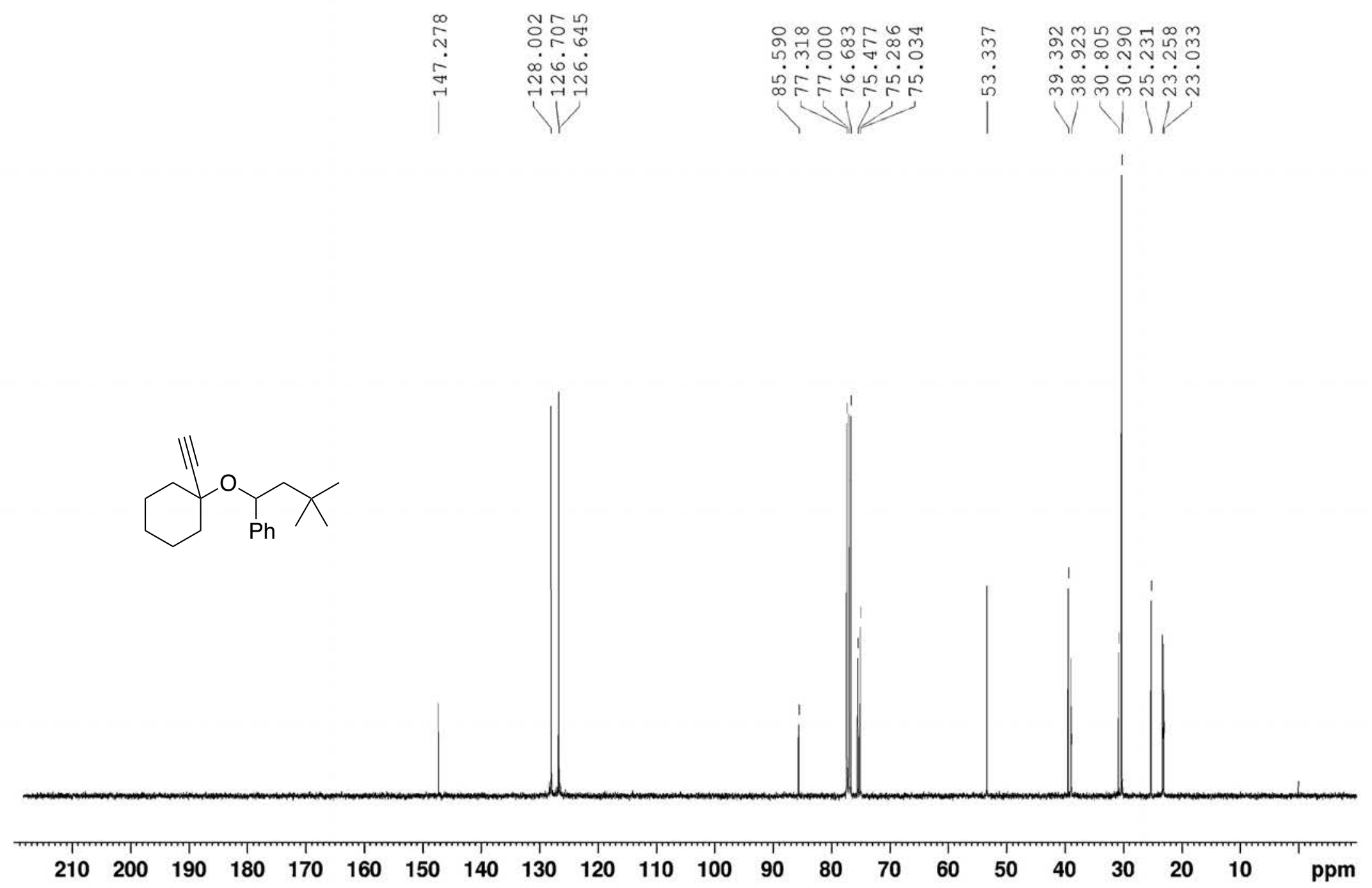

${ }^{13} \mathrm{C}$ NMR spectrum of 4 oaa $\left(100.6 \mathrm{MHz}, \mathrm{CDCl}_{3}\right)$ 


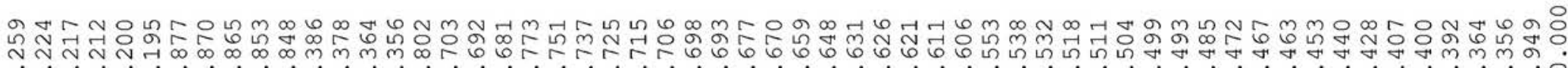

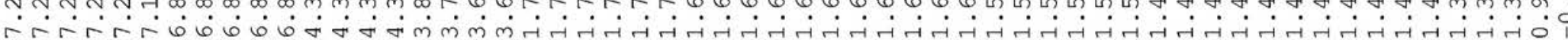

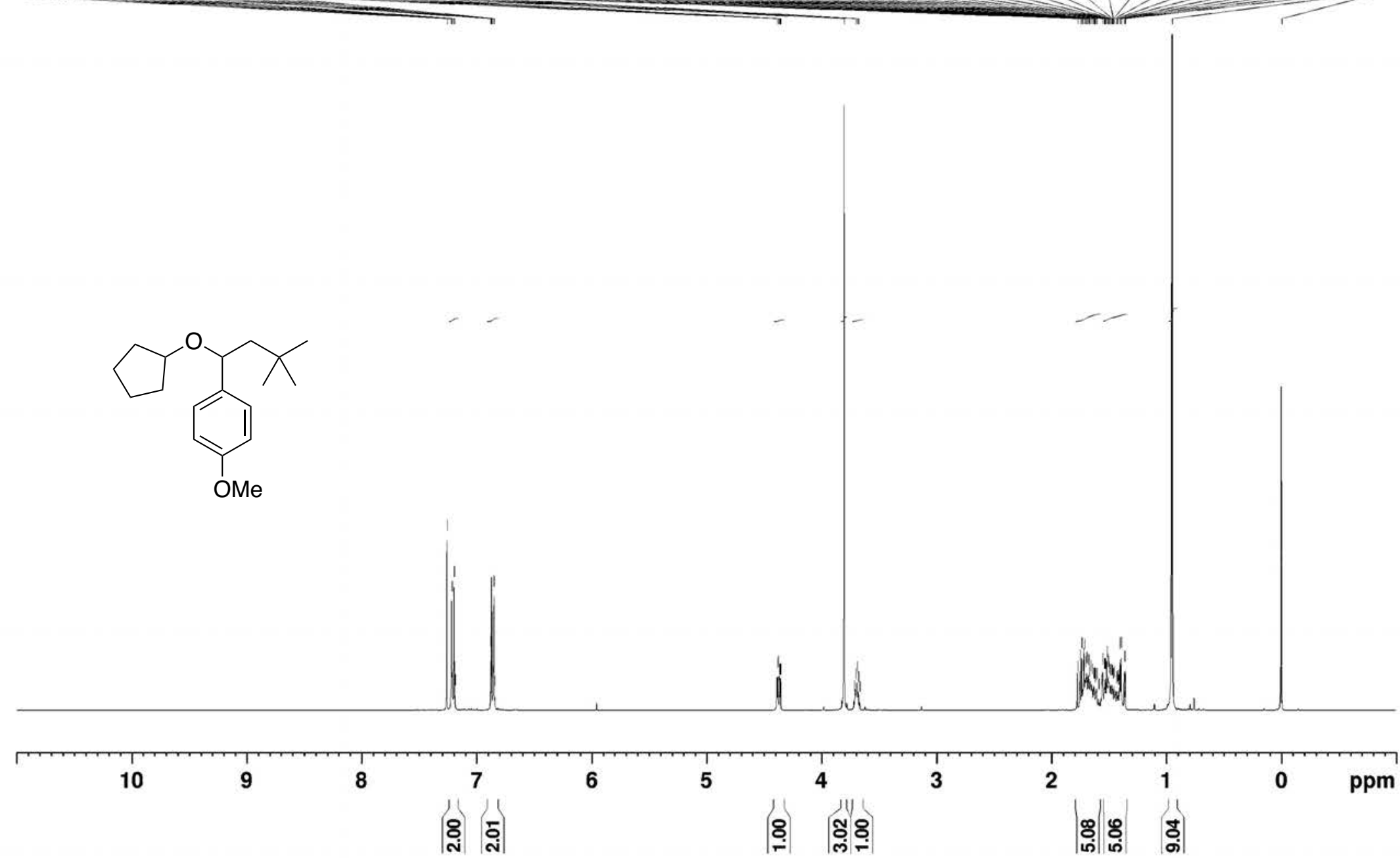

${ }^{1} \mathrm{H}$ NMR spectrum of $\mathbf{4 a b a}\left(400 \mathrm{MHz}, \mathrm{CDCl}_{3}\right)$ 


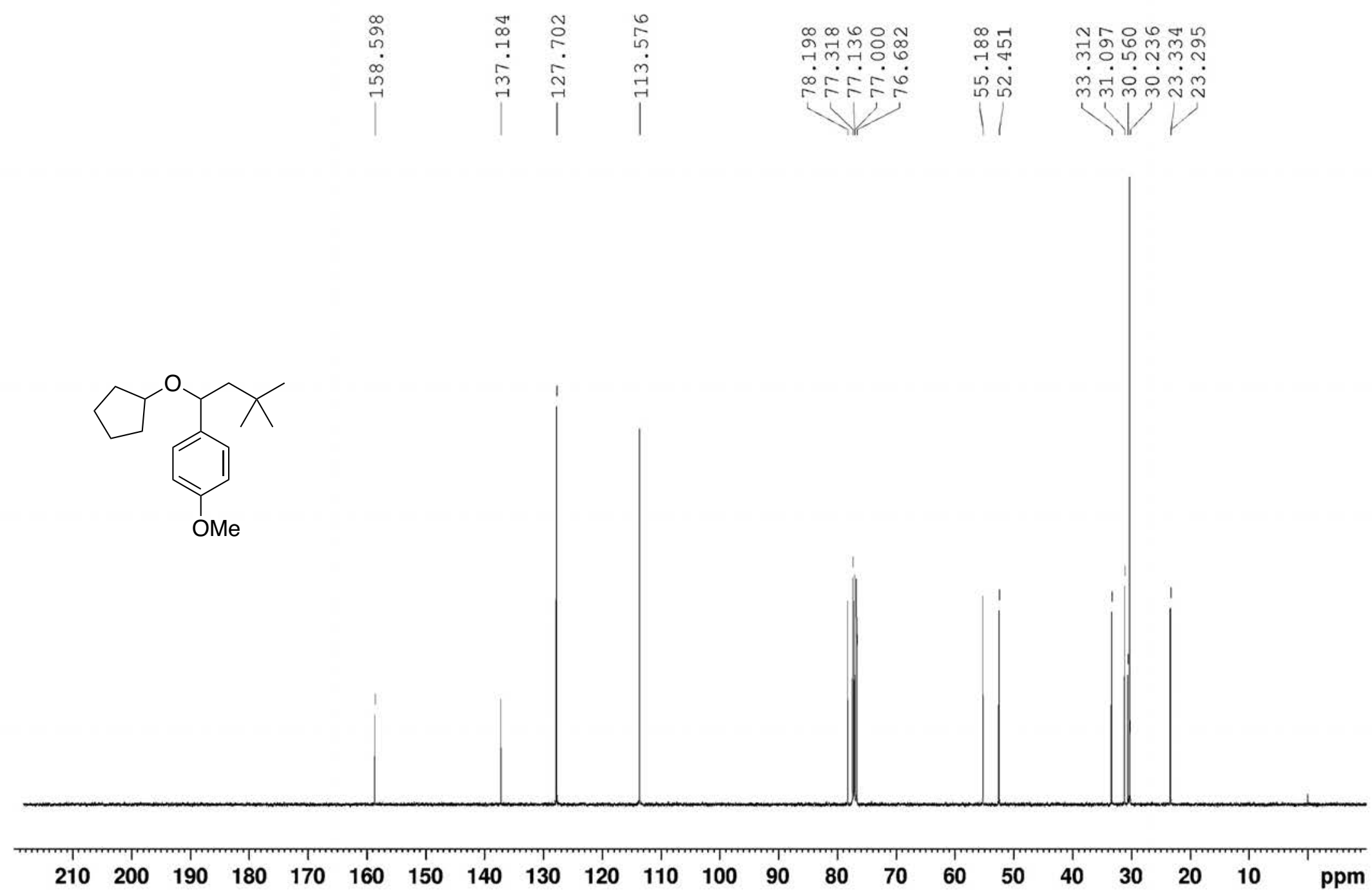

${ }^{13} \mathrm{C}$ NMR spectrum of $\mathbf{4 a b a}\left(100.6 \mathrm{MHz}, \mathrm{CDCl}_{3}\right)$ 


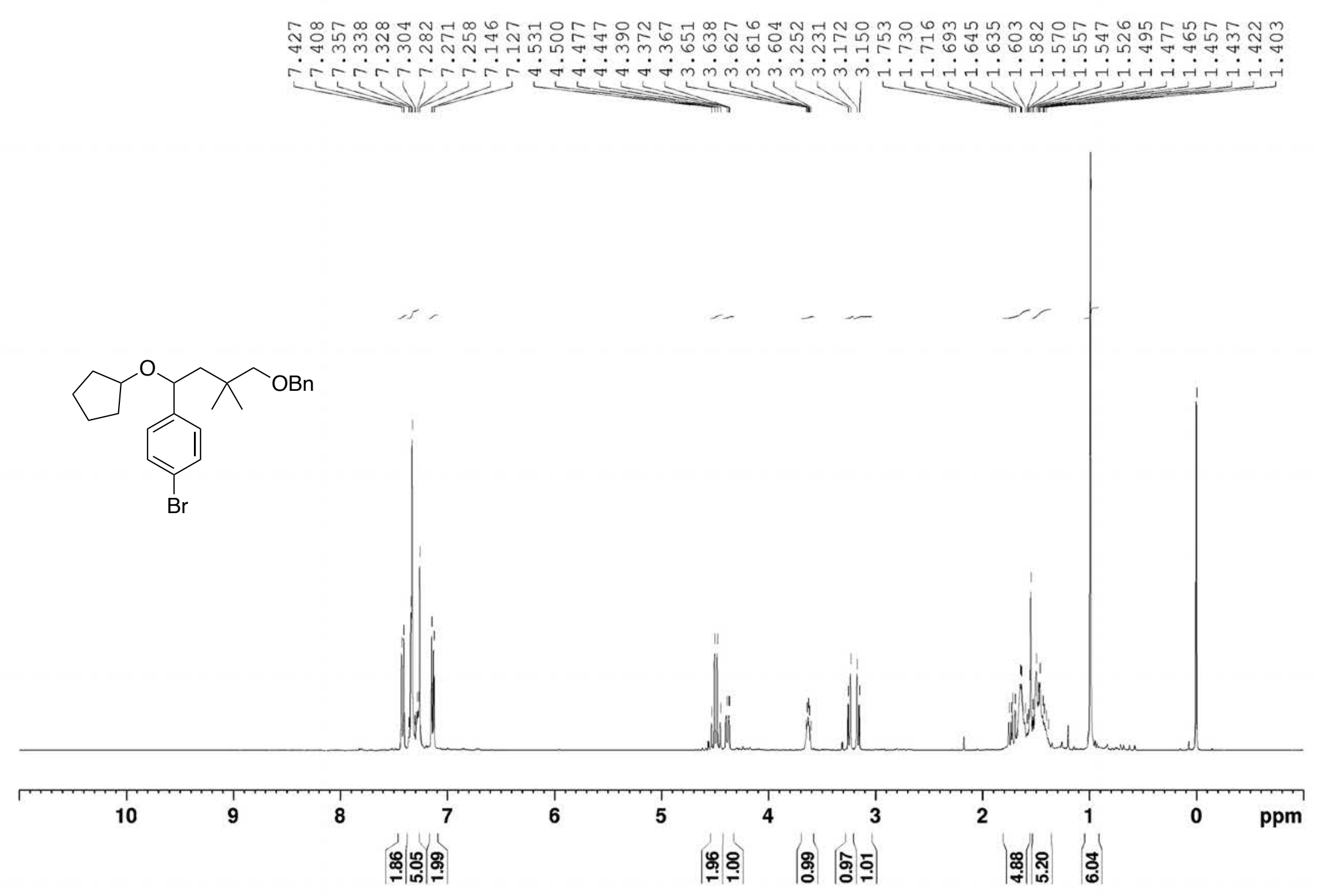

${ }^{1} \mathrm{H}$ NMR spectrum of 4 acc $\left(400 \mathrm{MHz}, \mathrm{CDCl}_{3}\right)$ 


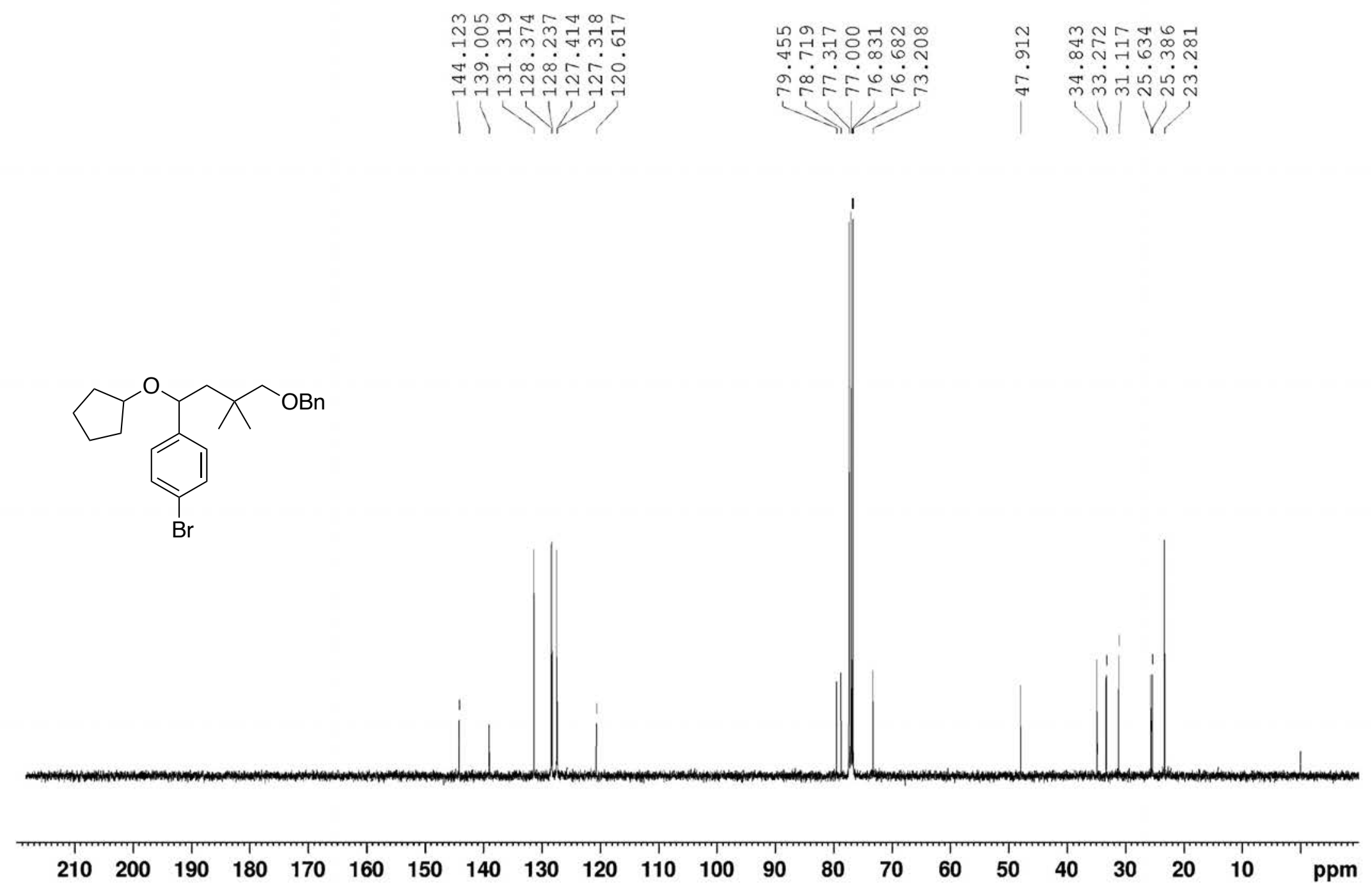

${ }^{13} \mathrm{C}$ NMR spectrum of $4 \operatorname{acc}\left(100.6 \mathrm{MHz}, \mathrm{CDCl}_{3}\right)$ 


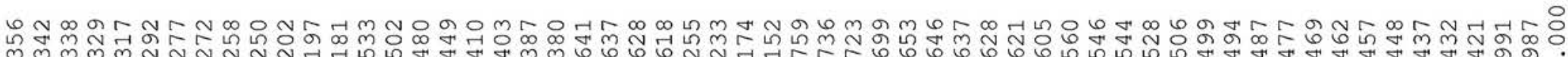

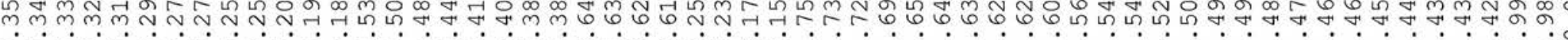

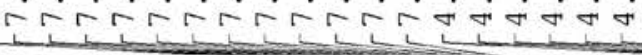

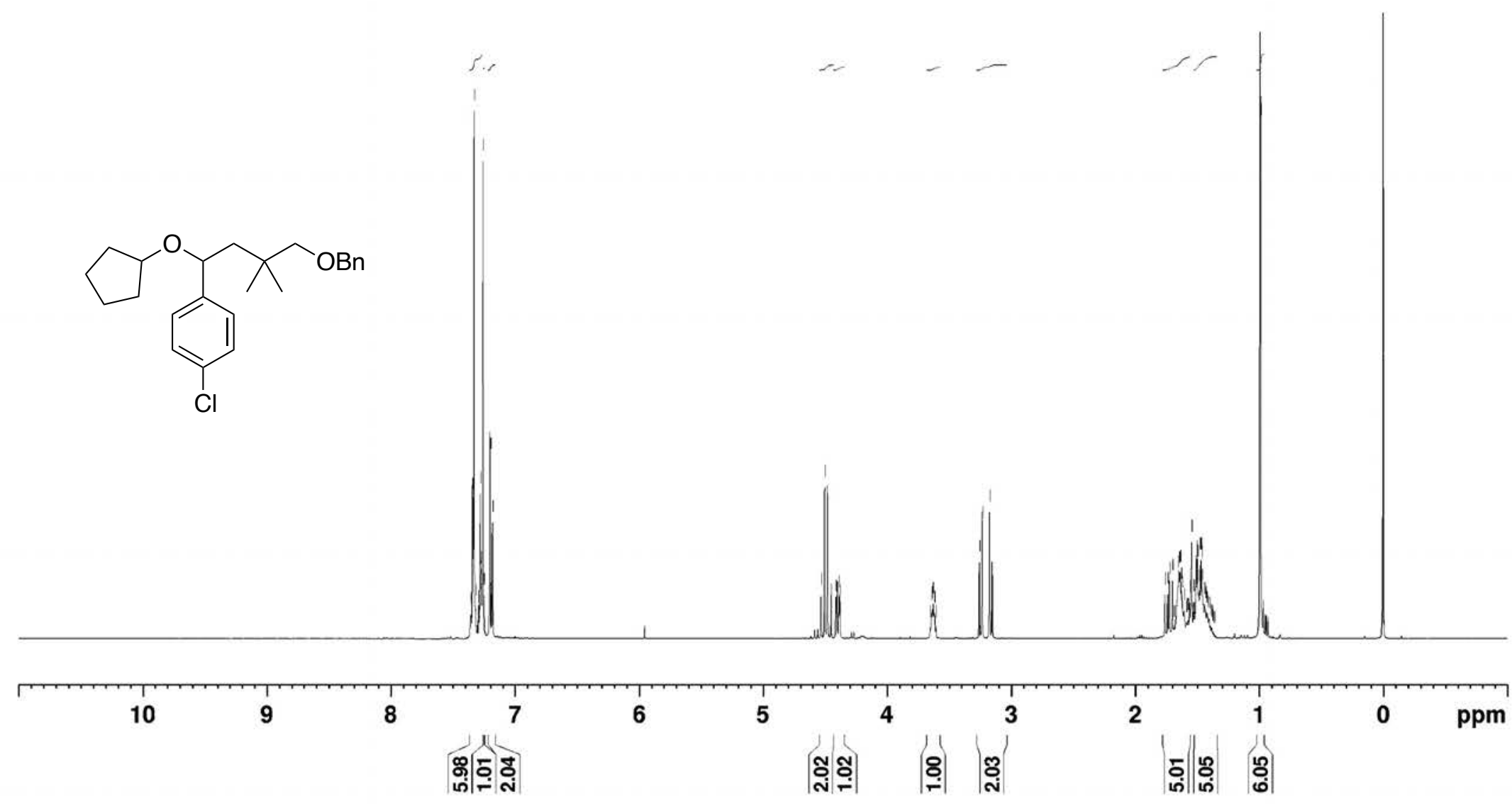

${ }^{1} \mathrm{H}$ NMR spectrum of 4 adc $\left(400 \mathrm{MHz}, \mathrm{CDCl}_{3}\right)$ 


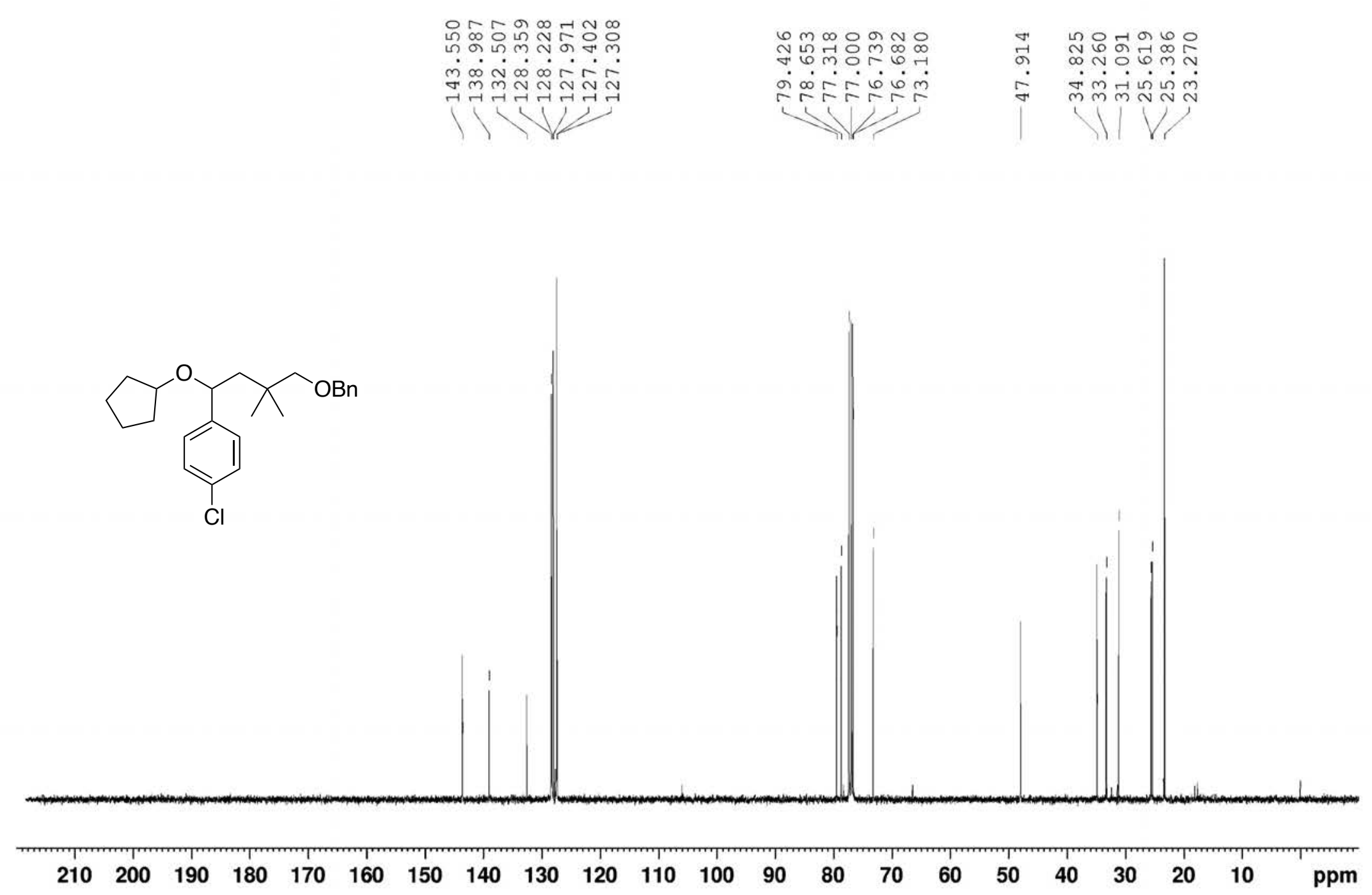

${ }^{13} \mathrm{C}$ NMR spectrum of 4 adc $\left(100.6 \mathrm{MHz}, \mathrm{CDCl}_{3}\right)$ 


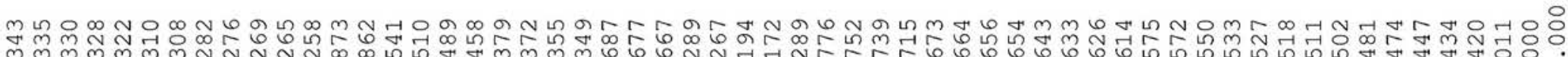

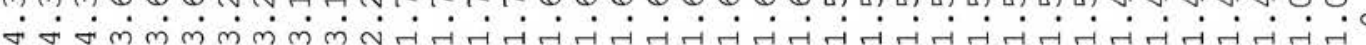

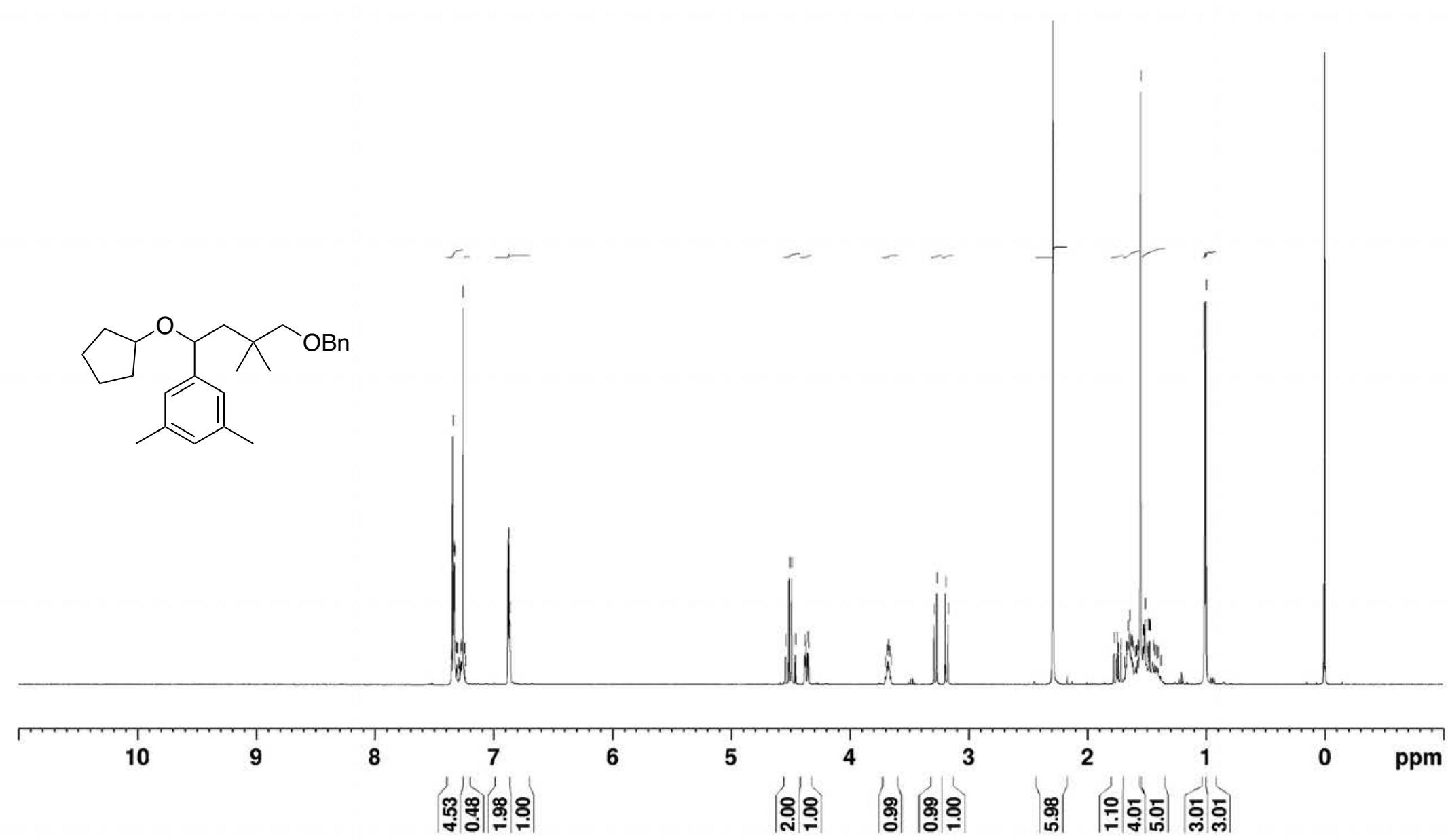

${ }^{1} \mathrm{H}$ NMR spectrum of 4 aec $\left(400 \mathrm{MHz}, \mathrm{CDCl}_{3}\right)$ 


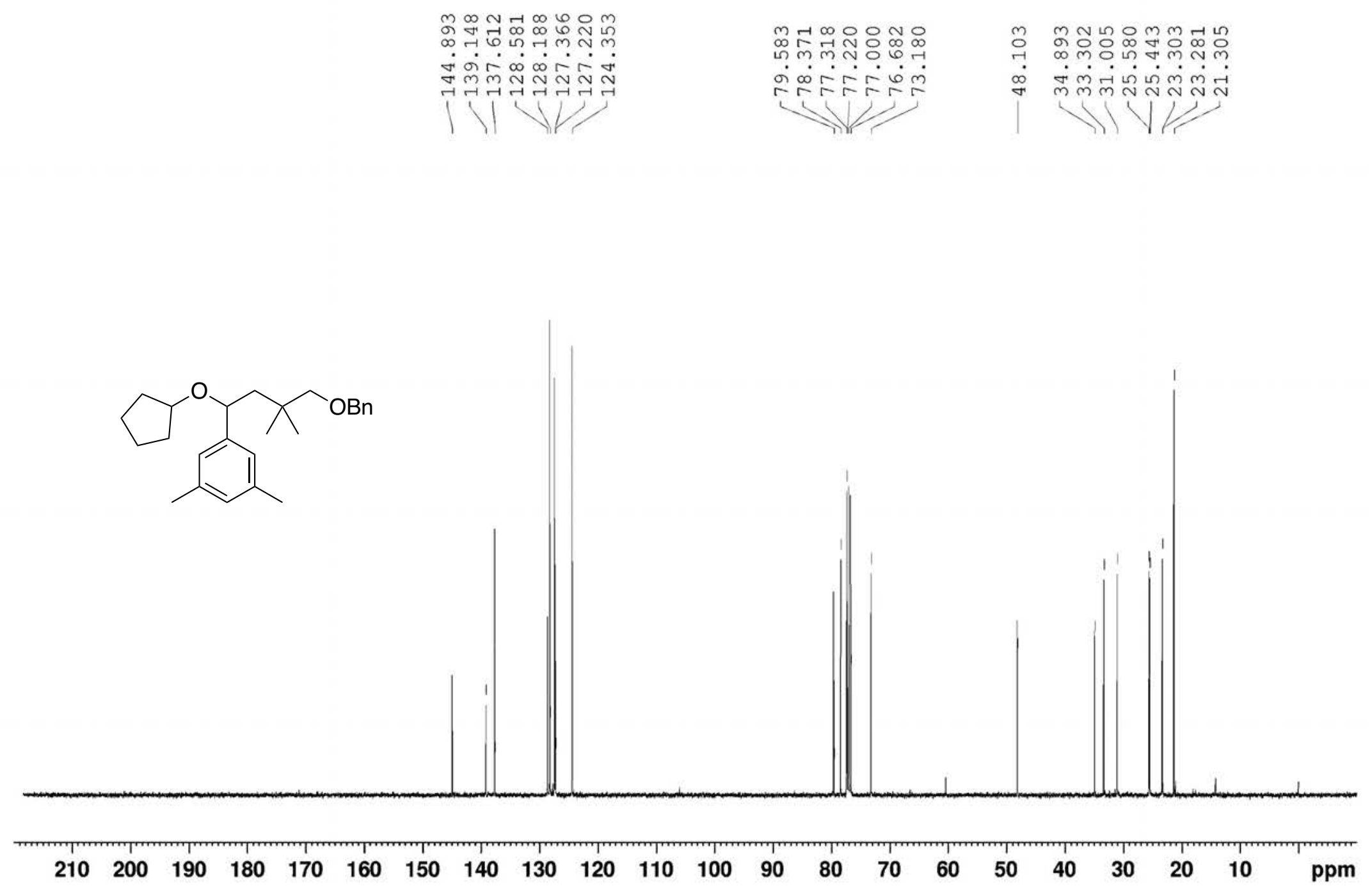

${ }^{13} \mathrm{C}$ NMR spectrum of $4 \mathbf{a e c}\left(100.6 \mathrm{MHz}, \mathrm{CDCl}_{3}\right)$ 


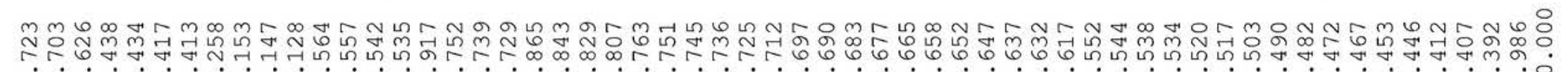
rirriि,

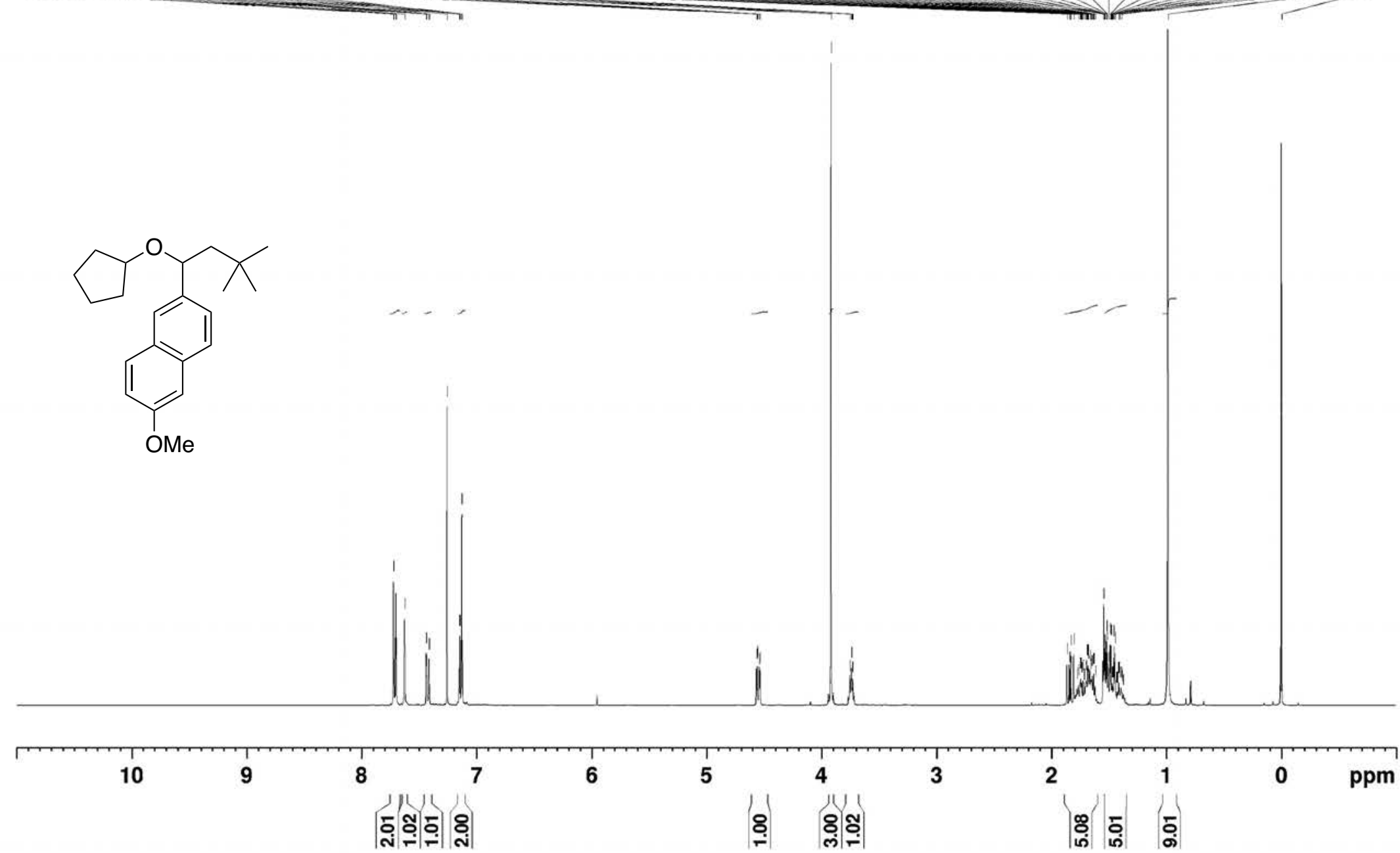

${ }^{1} \mathrm{H}$ NMR spectrum of 4 afa $\left(400 \mathrm{MHz}, \mathrm{CDCl}_{3}\right)$ 


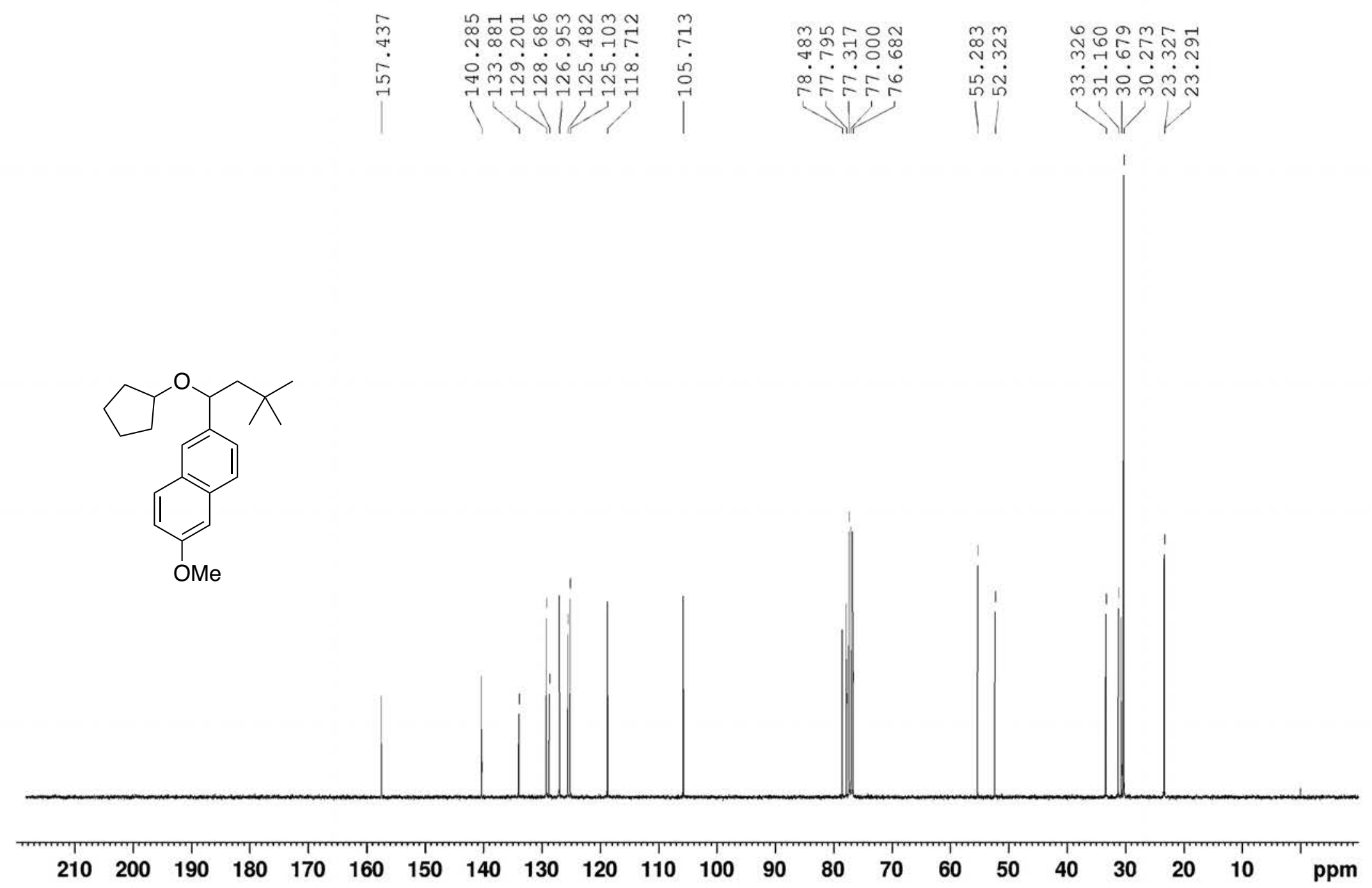

${ }^{13} \mathrm{C}$ NMR spectrum of $\mathbf{4 a f a}\left(100.6 \mathrm{MHz}, \mathrm{CDCl}_{3}\right)$ 


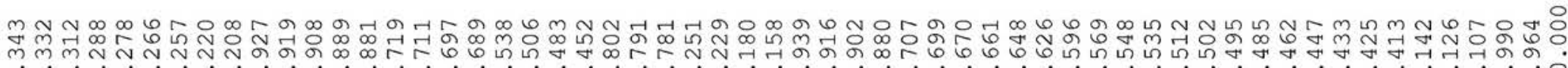

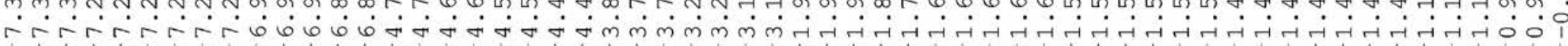

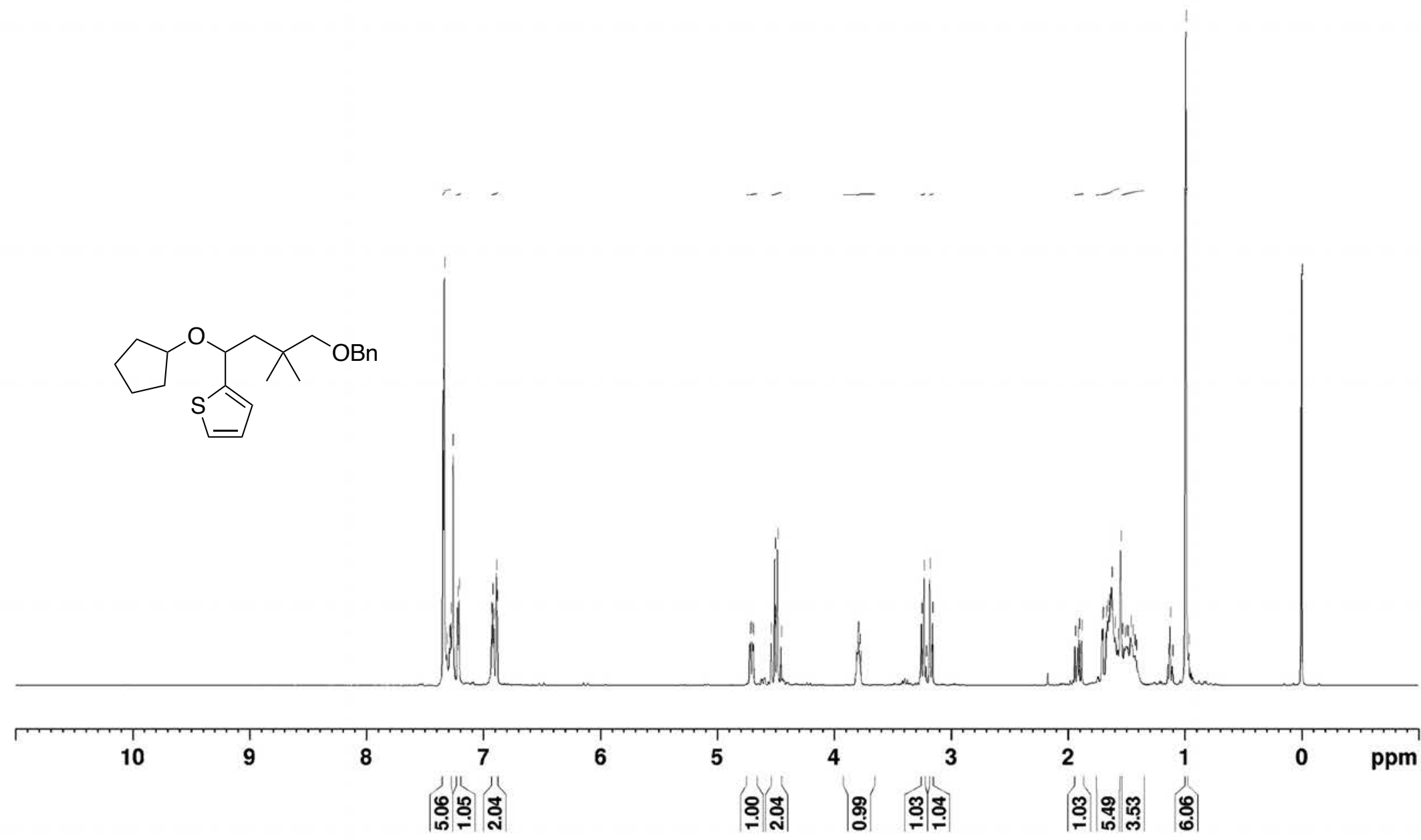

${ }^{1} \mathrm{H}$ NMR spectrum of 4 agc $\left(400 \mathrm{MHz}, \mathrm{CDCl}_{3}\right)$ 


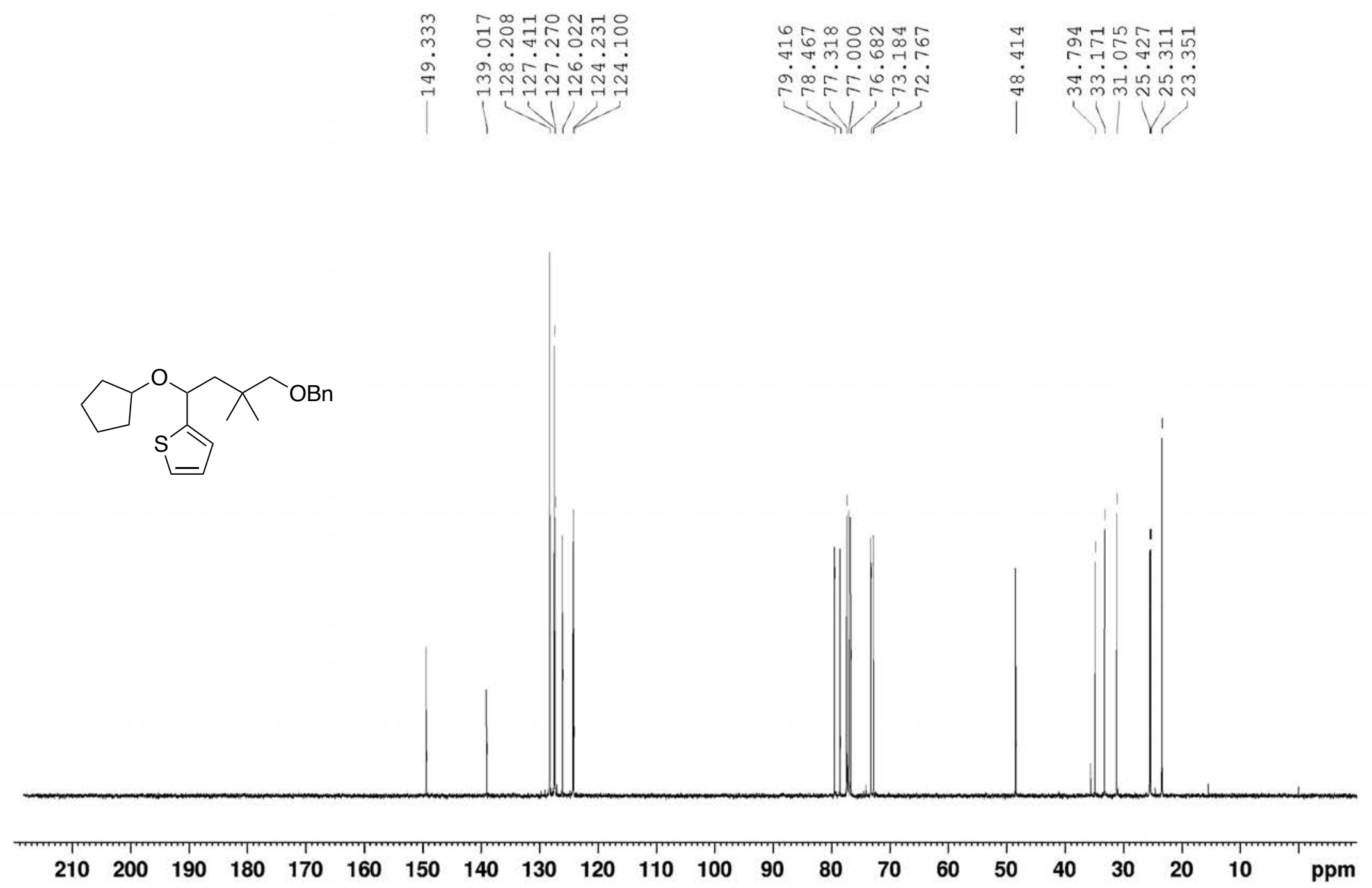

${ }^{13} \mathrm{C}$ NMR spectrum of 4 age $\left(100.6 \mathrm{MHz}, \mathrm{CDCl}_{3}\right)$ 


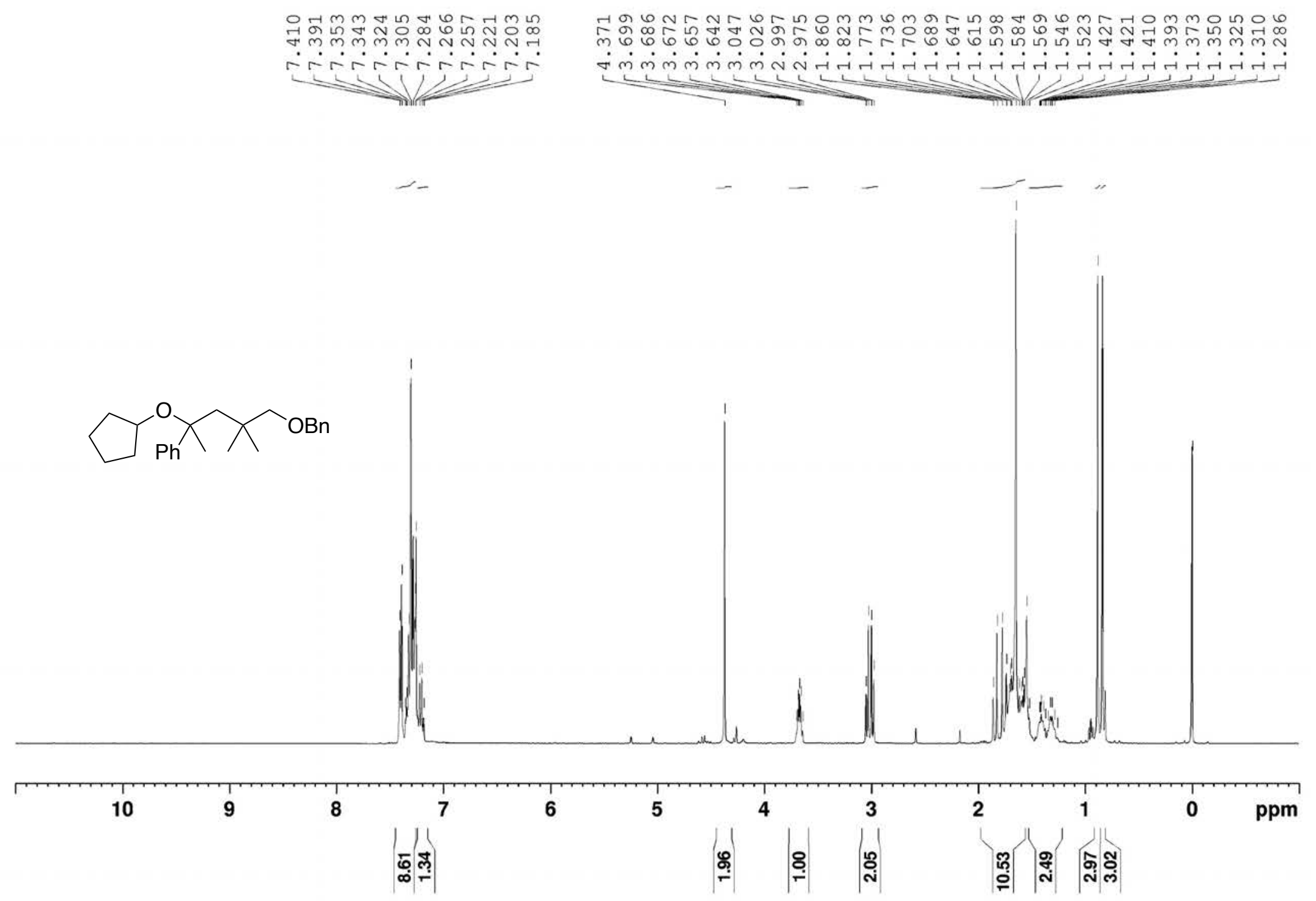

${ }^{1} \mathrm{H}$ NMR spectrum of $\mathbf{4 a h c}\left(400 \mathrm{MHz}, \mathrm{CDCl}_{3}\right)$ 


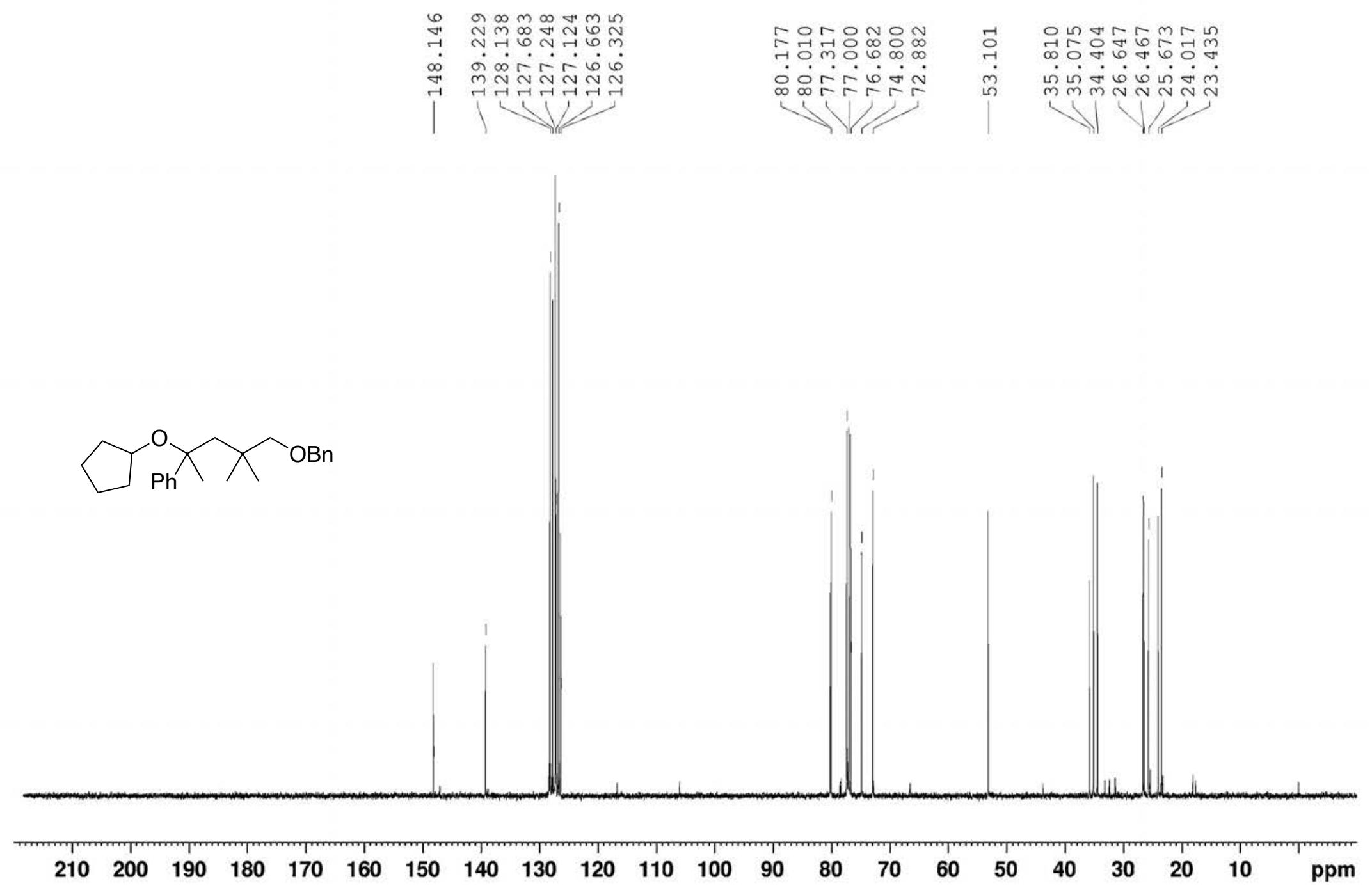

${ }^{13} \mathrm{C}$ NMR spectrum of $\mathbf{4 a h c}\left(100.6 \mathrm{MHz}, \mathrm{CDCl}_{3}\right)$ 


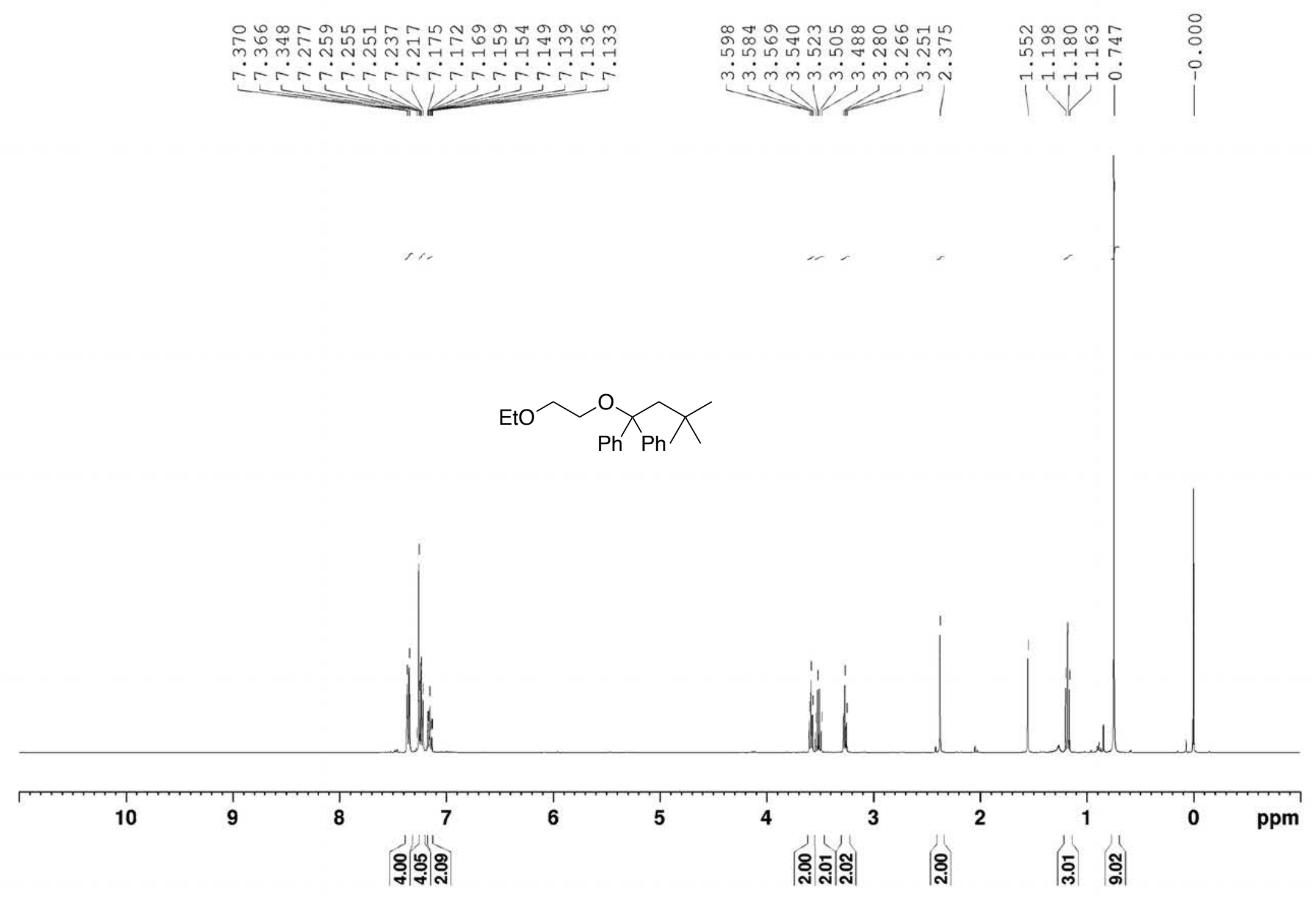

${ }^{1} \mathrm{H}$ NMR spectrum of 4 dia $\left(400 \mathrm{MHz}, \mathrm{CDCl}_{3}\right)$ 


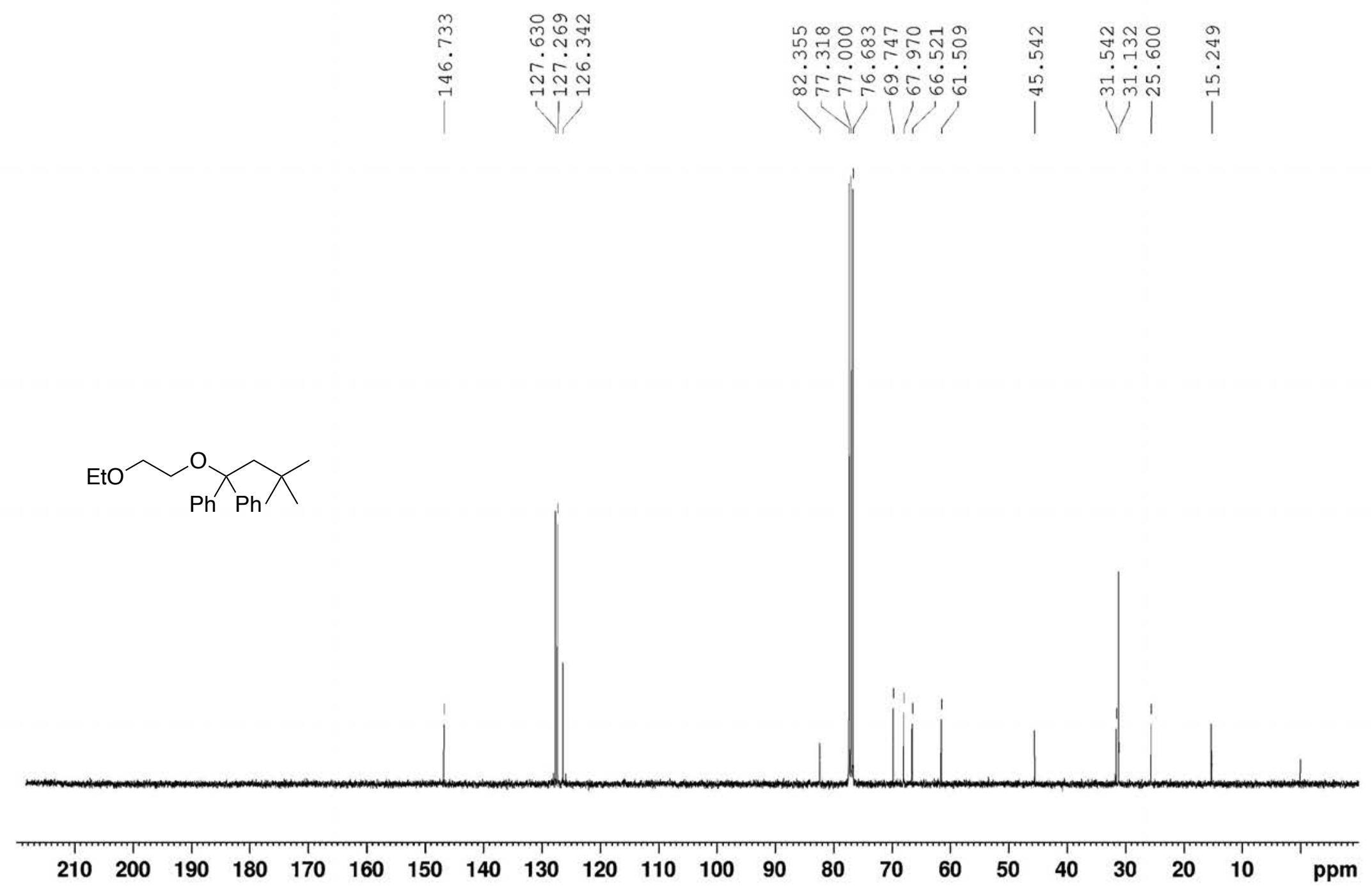

${ }^{13} \mathrm{C}$ NMR spectrum of 4 dia $\left(100.6 \mathrm{MHz}, \mathrm{CDCl}_{3}\right)$ 
क ॠ

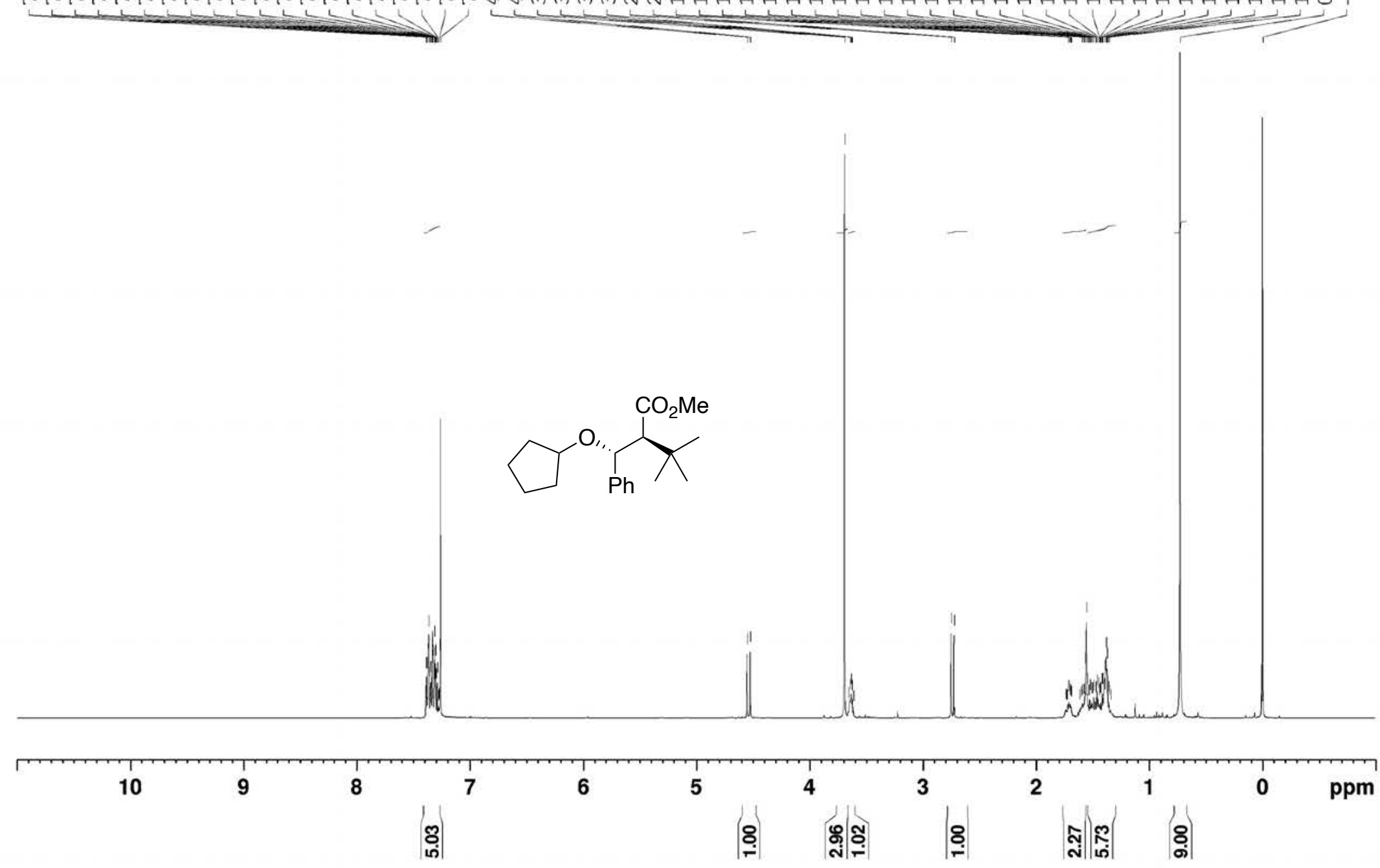

${ }^{1} \mathrm{H}$ NMR spectrum of 4aja (400 MHz, $\mathrm{CDCl}_{3}$ ) 

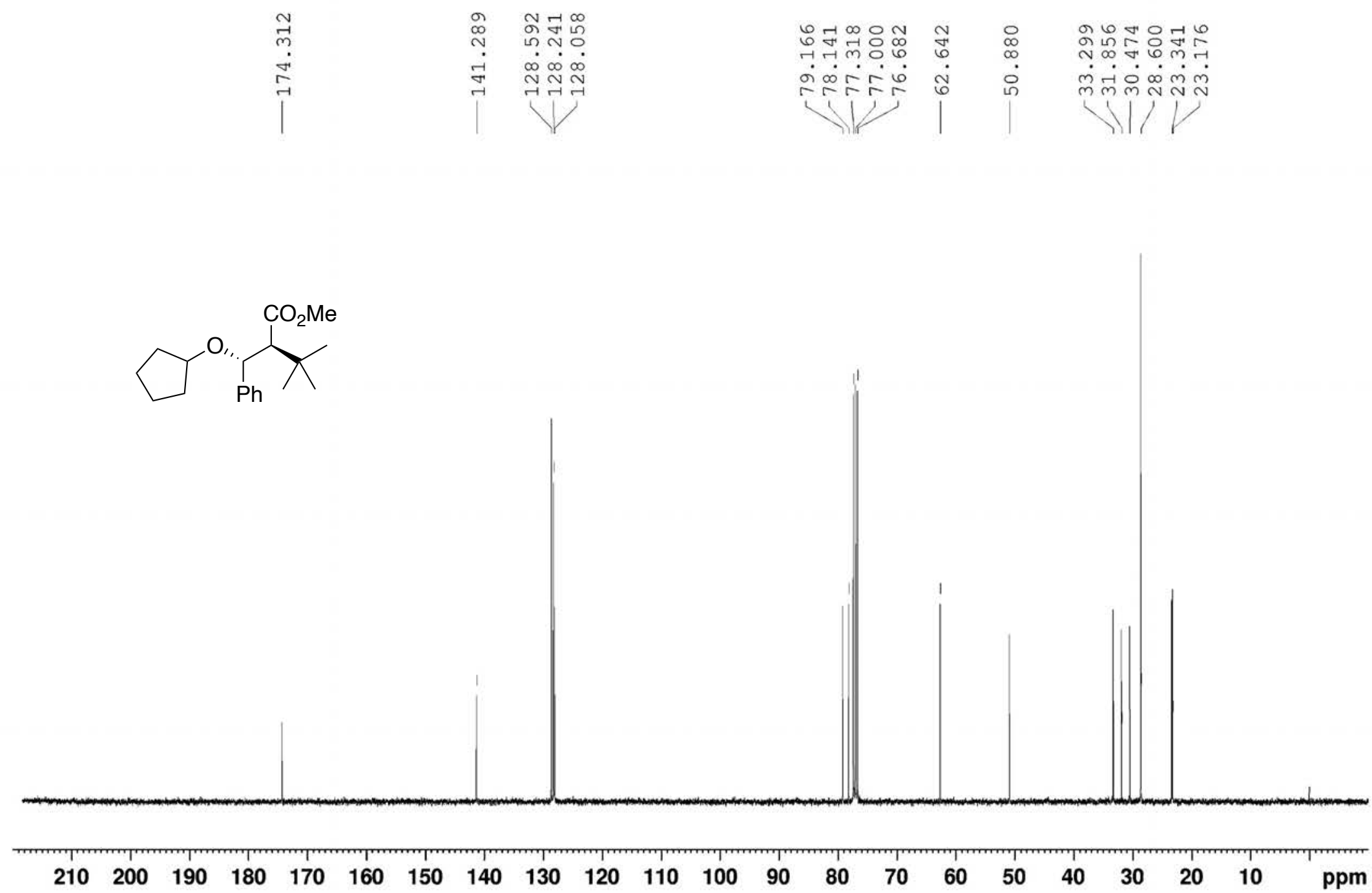

${ }^{13} \mathrm{C}$ NMR spectrum of 4aja $\left(100.6 \mathrm{MHz}, \mathrm{CDCl}_{3}\right)$ 


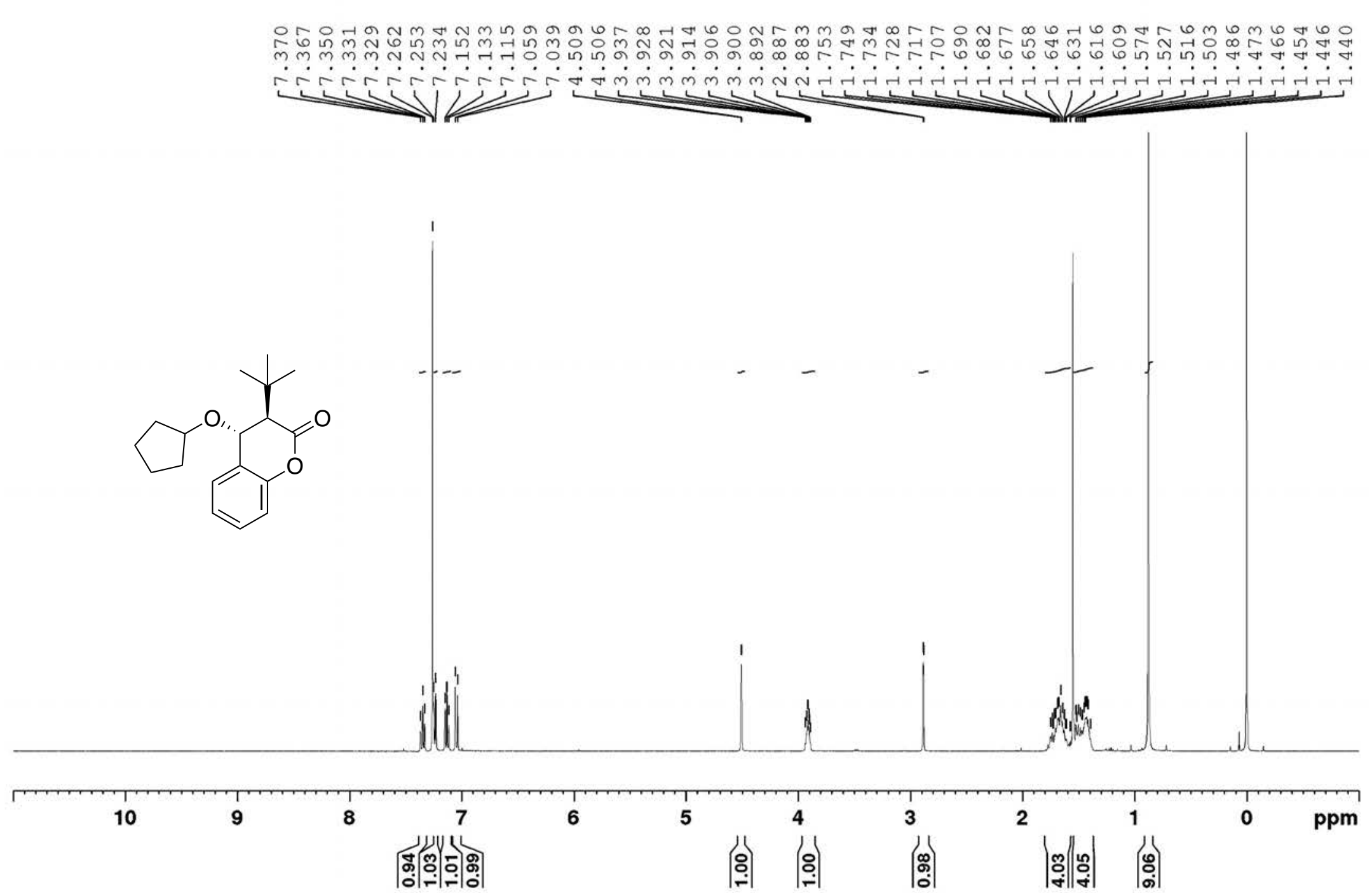

${ }^{1} \mathrm{H}$ NMR spectrum of $\mathbf{4 a k a}\left(400 \mathrm{MHz}, \mathrm{CDCl}_{3}\right.$ ) 


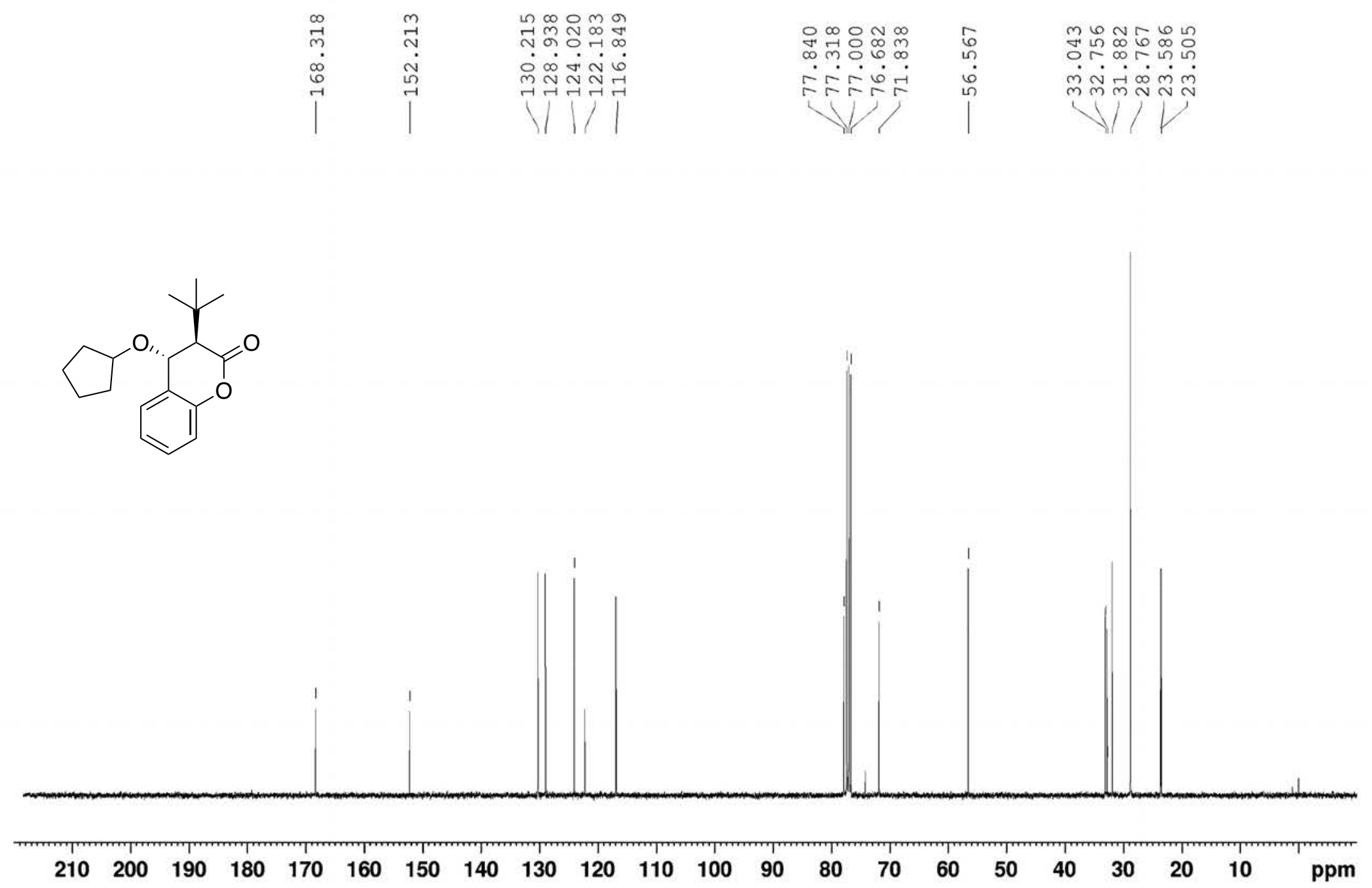

${ }^{13} \mathrm{C}$ NMR spectrum of $\mathbf{4 a k a}\left(100.6 \mathrm{MHz}, \mathrm{CDCl}_{3}\right)$ 


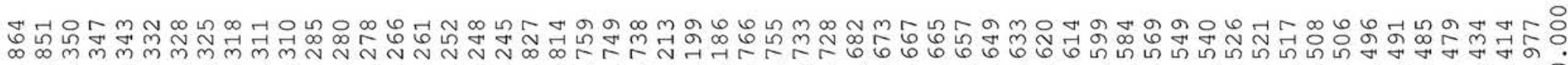

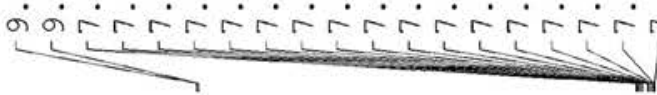

mmmnNN $m$ m

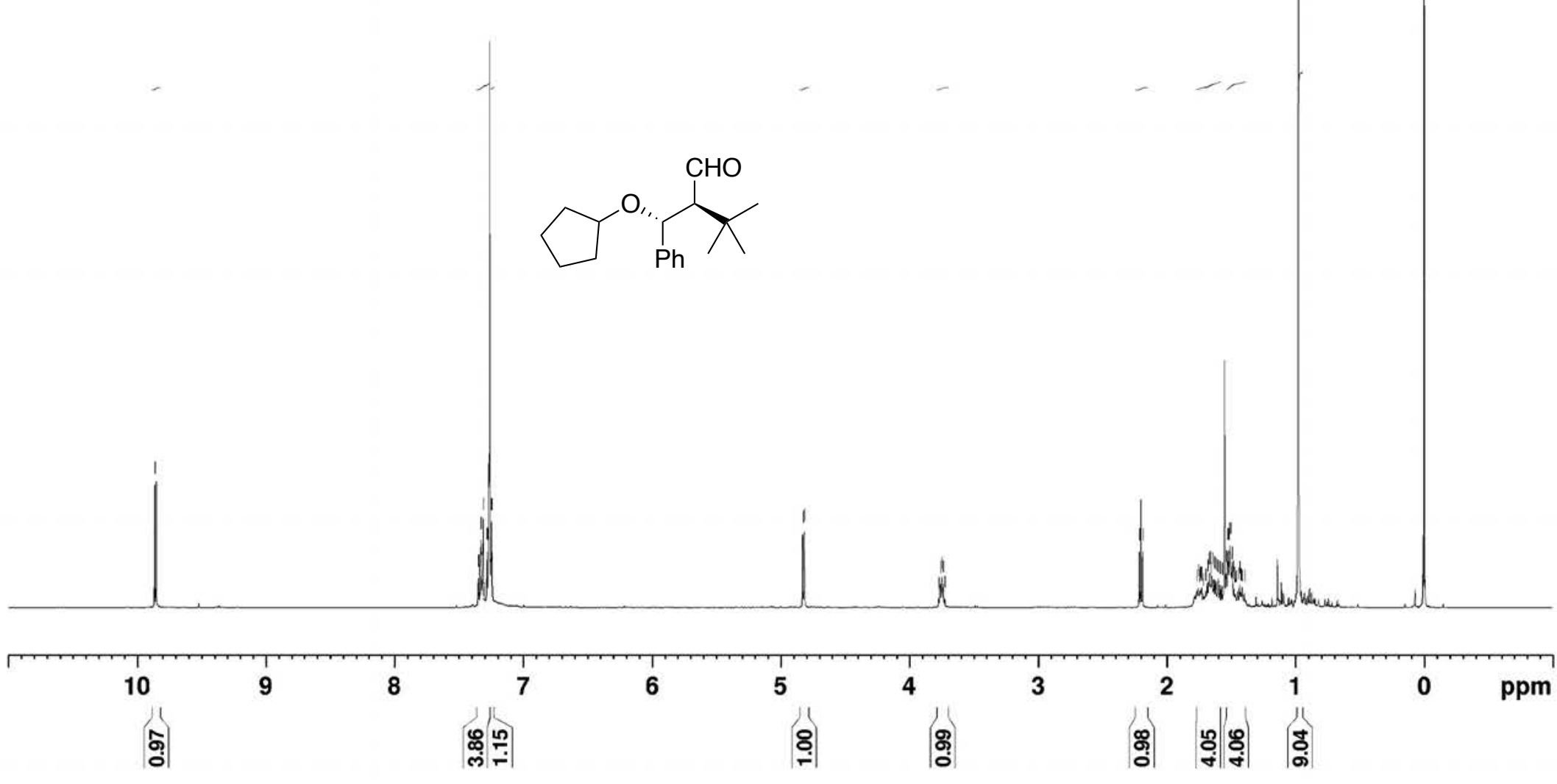

${ }^{1} \mathrm{H}$ NMR spectrum of 4 ala $\left(400 \mathrm{MHz}, \mathrm{CDCl}_{3}\right)$ 

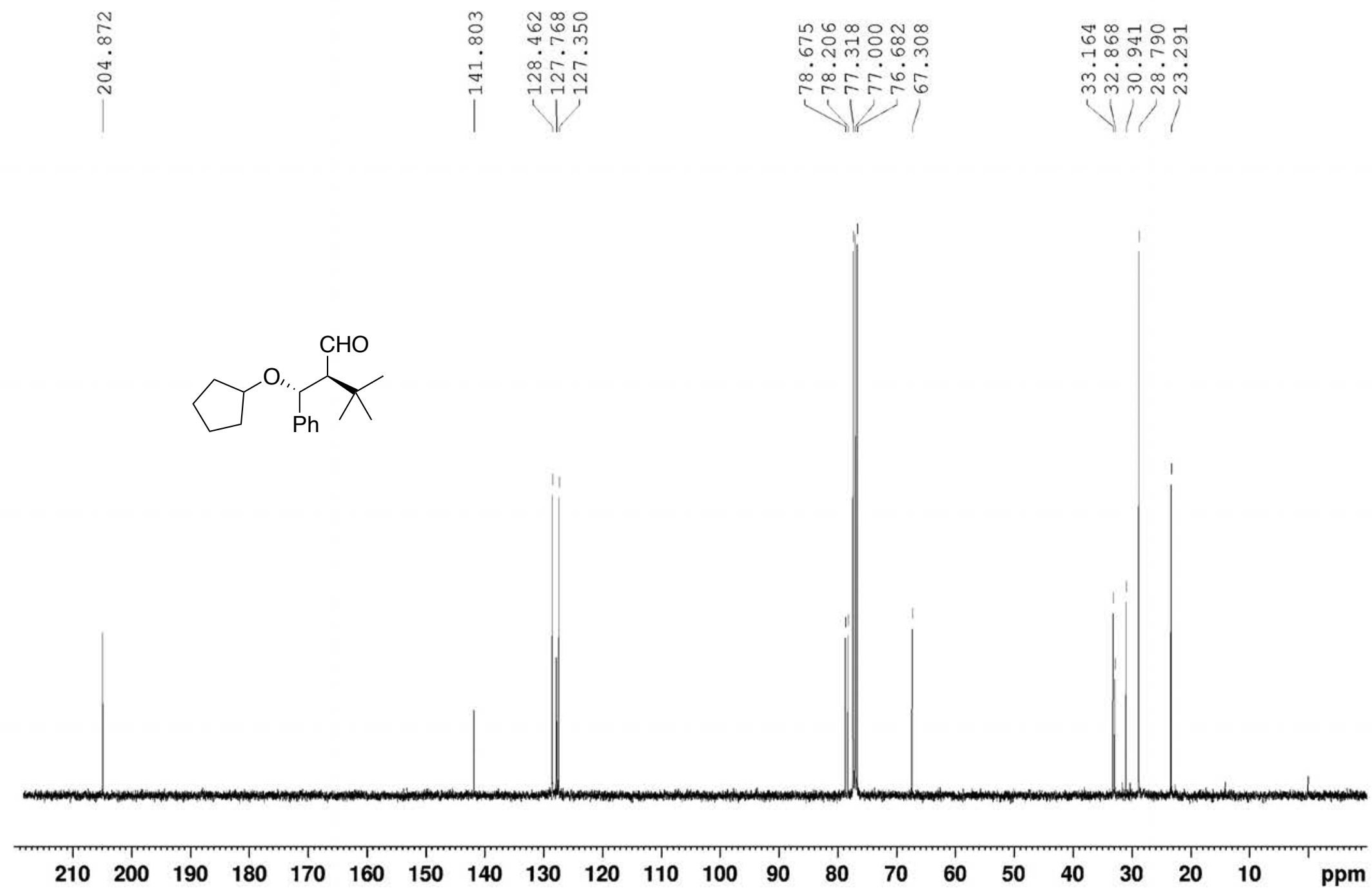

${ }^{13} \mathrm{C}$ NMR spectrum of 4 ala $\left(100.6 \mathrm{MHz}, \mathrm{CDCl}_{3}\right)$ 


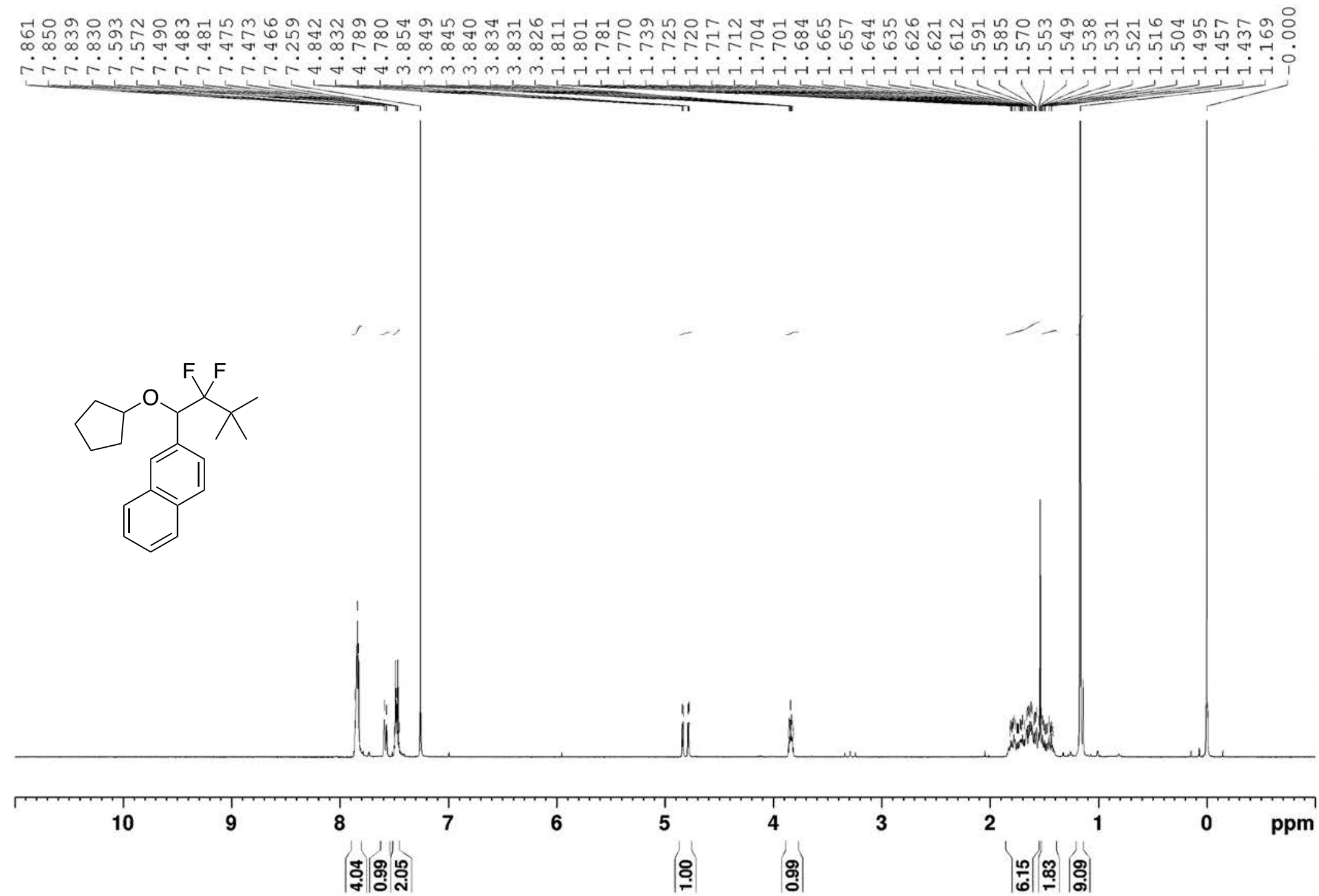

${ }^{1} \mathrm{H}$ NMR spectrum of $4 \mathbf{a m a}\left(400 \mathrm{MHz}, \mathrm{CDCl}_{3}\right.$ ) 

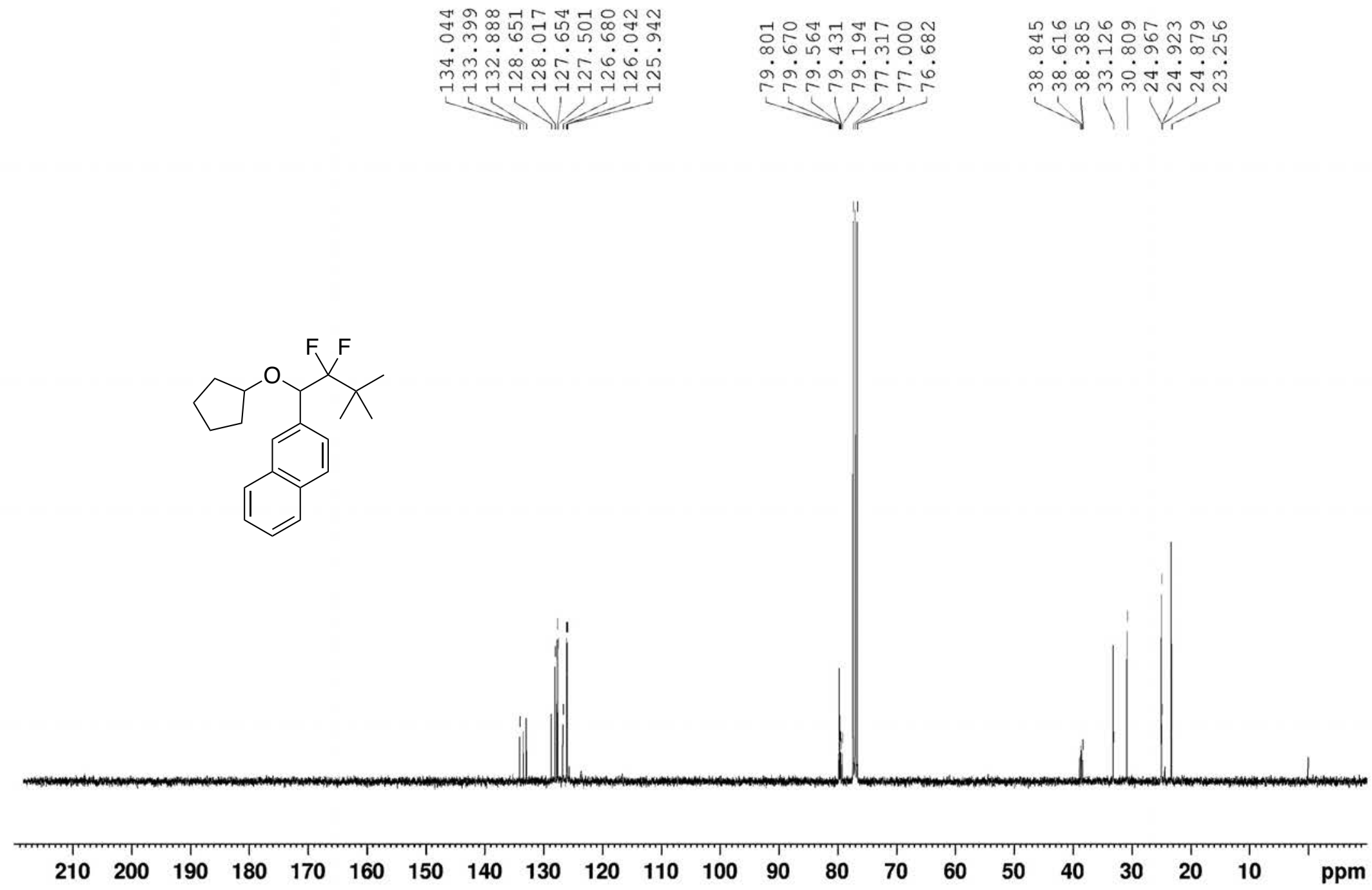

${ }^{13} \mathrm{C}$ NMR spectrum of 4 ama $\left(100.6 \mathrm{MHz}, \mathrm{CDCl}_{3}\right)$ 


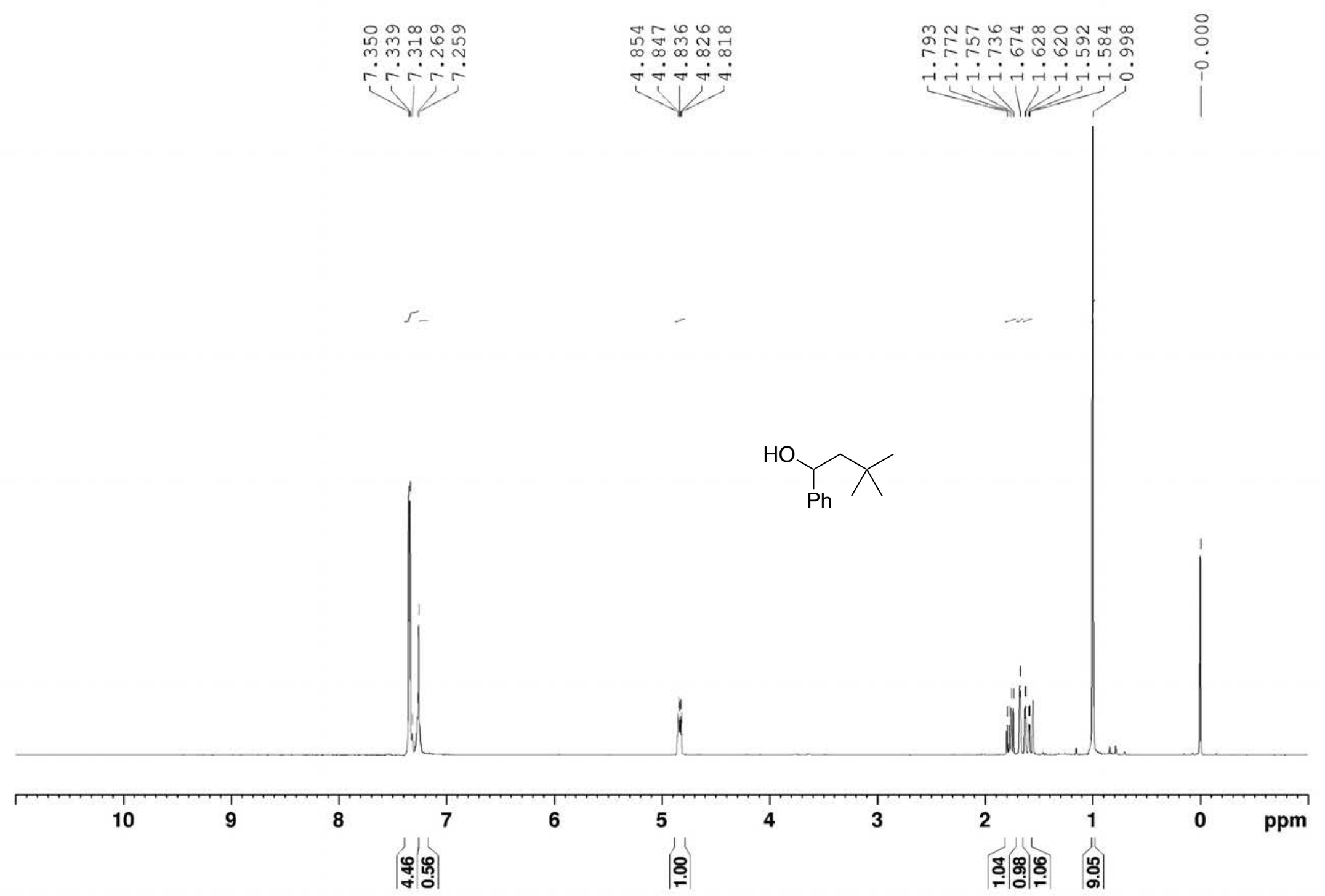

${ }^{1} \mathrm{H}$ NMR spectrum of $\mathbf{1 0 a a a}\left(400 \mathrm{MHz}, \mathrm{CDCl}_{3}\right.$ ) 


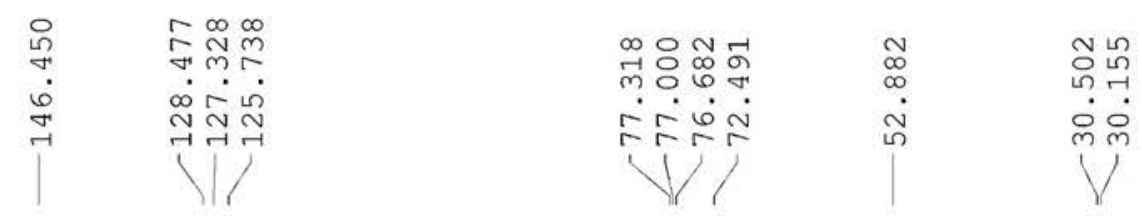

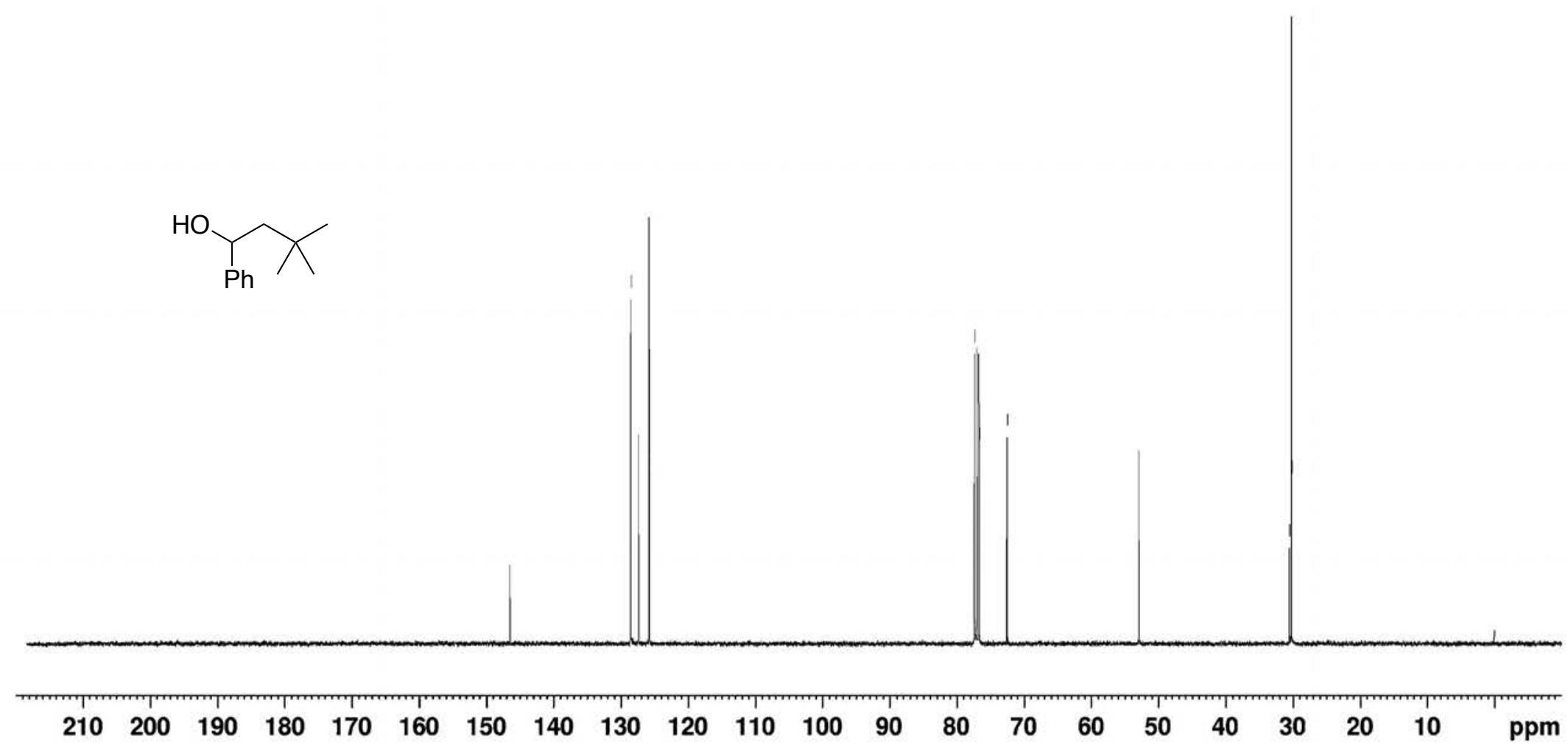

${ }^{13} \mathrm{C}$ NMR spectrum of $\mathbf{1 0 a a a}\left(100.6 \mathrm{MHz}, \mathrm{CDCl}_{3}\right)$ 


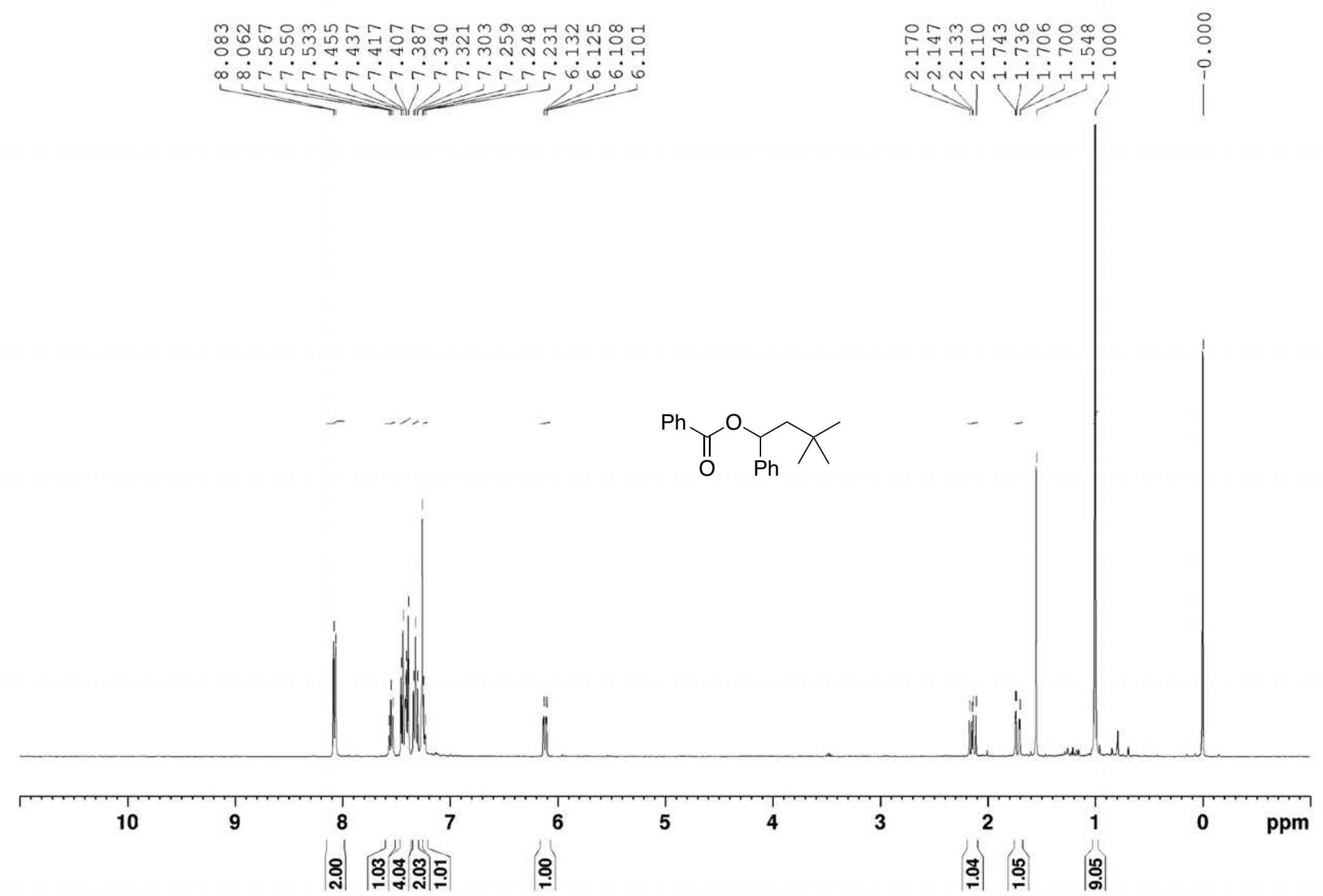

${ }^{1} \mathrm{H}$ NMR spectrum of 11 aaa (400 MHz, $\mathrm{CDCl}_{3}$ ) 


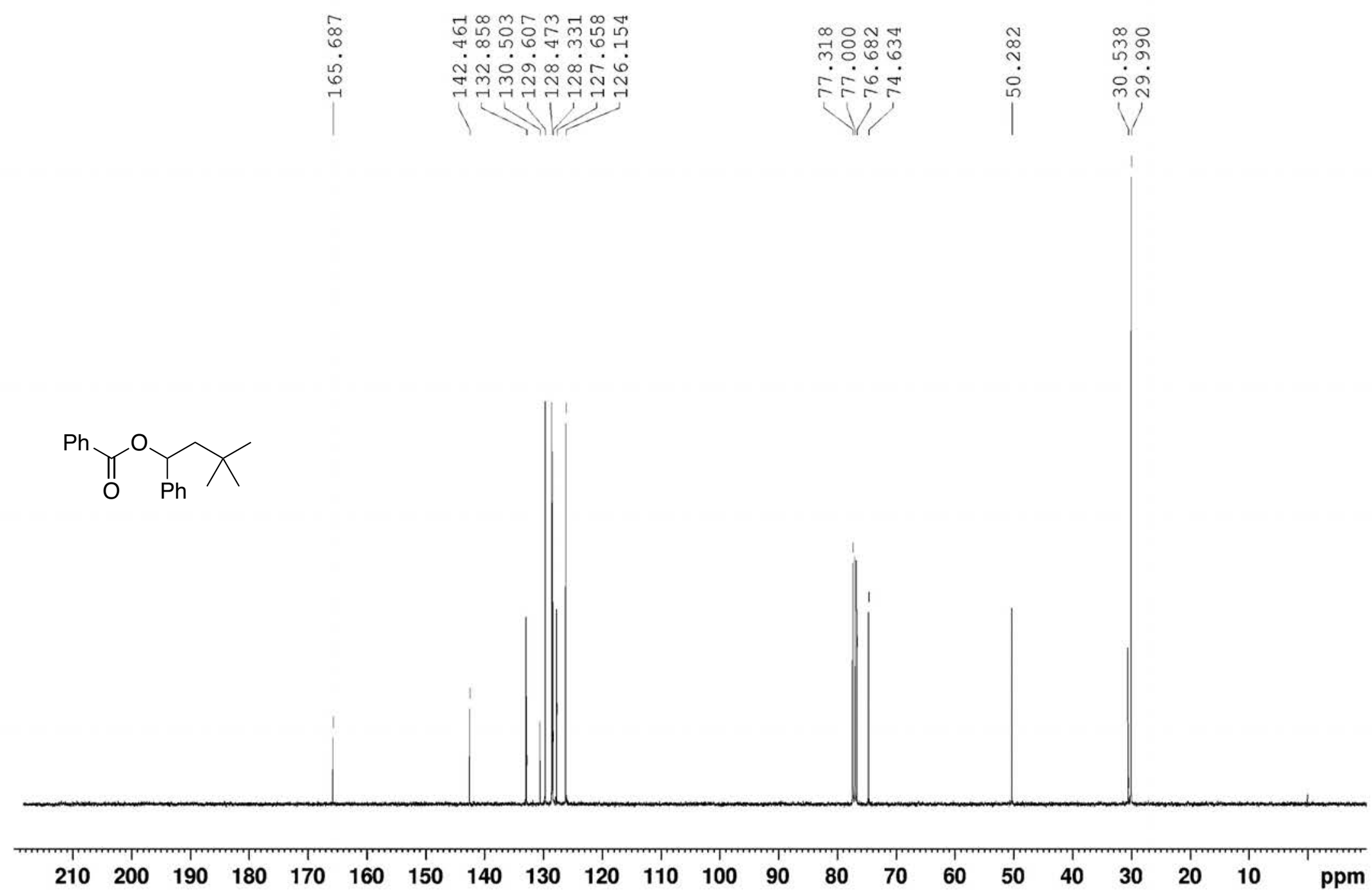

${ }^{13} \mathrm{C}$ NMR spectrum of $\mathbf{1 1 a a a}\left(100.6 \mathrm{MHz}, \mathrm{CDCl}_{3}\right)$ 


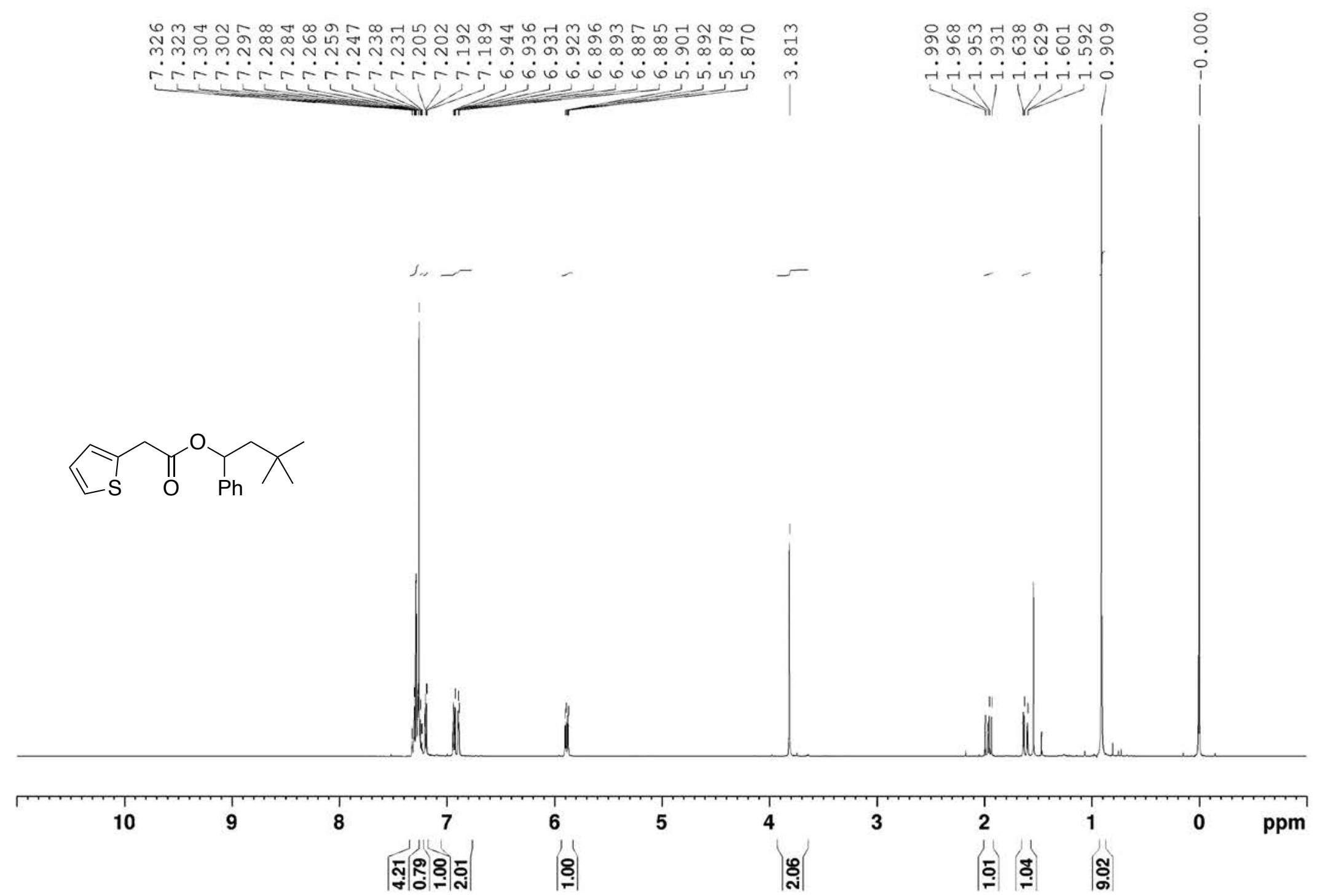

${ }^{1} \mathrm{H}$ NMR spectrum of $\mathbf{1 1} \mathbf{b a a}\left(400 \mathrm{MHz}, \mathrm{CDCl}_{3}\right.$ ) 


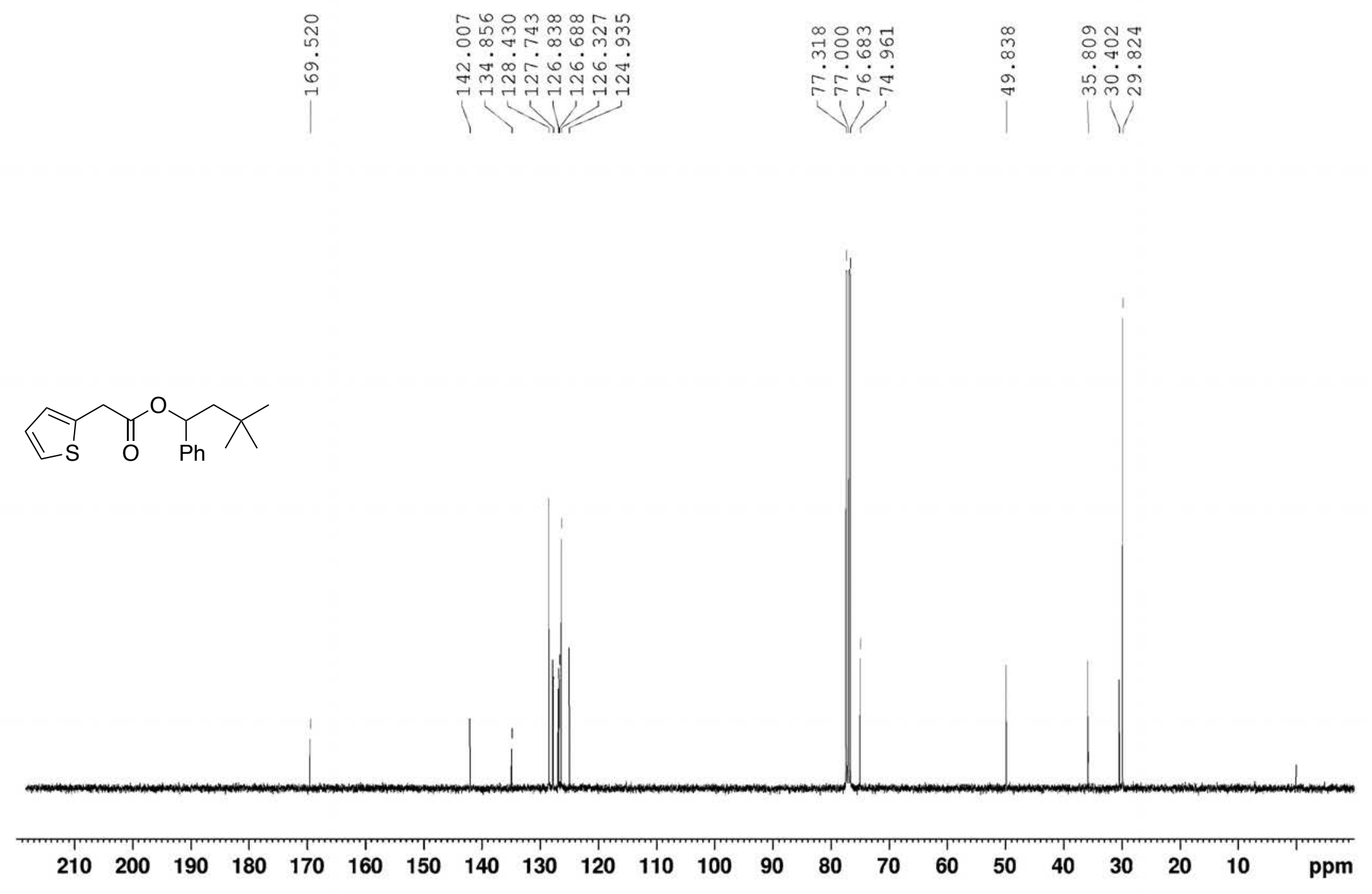

${ }^{13} \mathrm{C}$ NMR spectrum of $\mathbf{1 1}$ baa $\left(100.6 \mathrm{MHz}, \mathrm{CDCl}_{3}\right.$ ) 


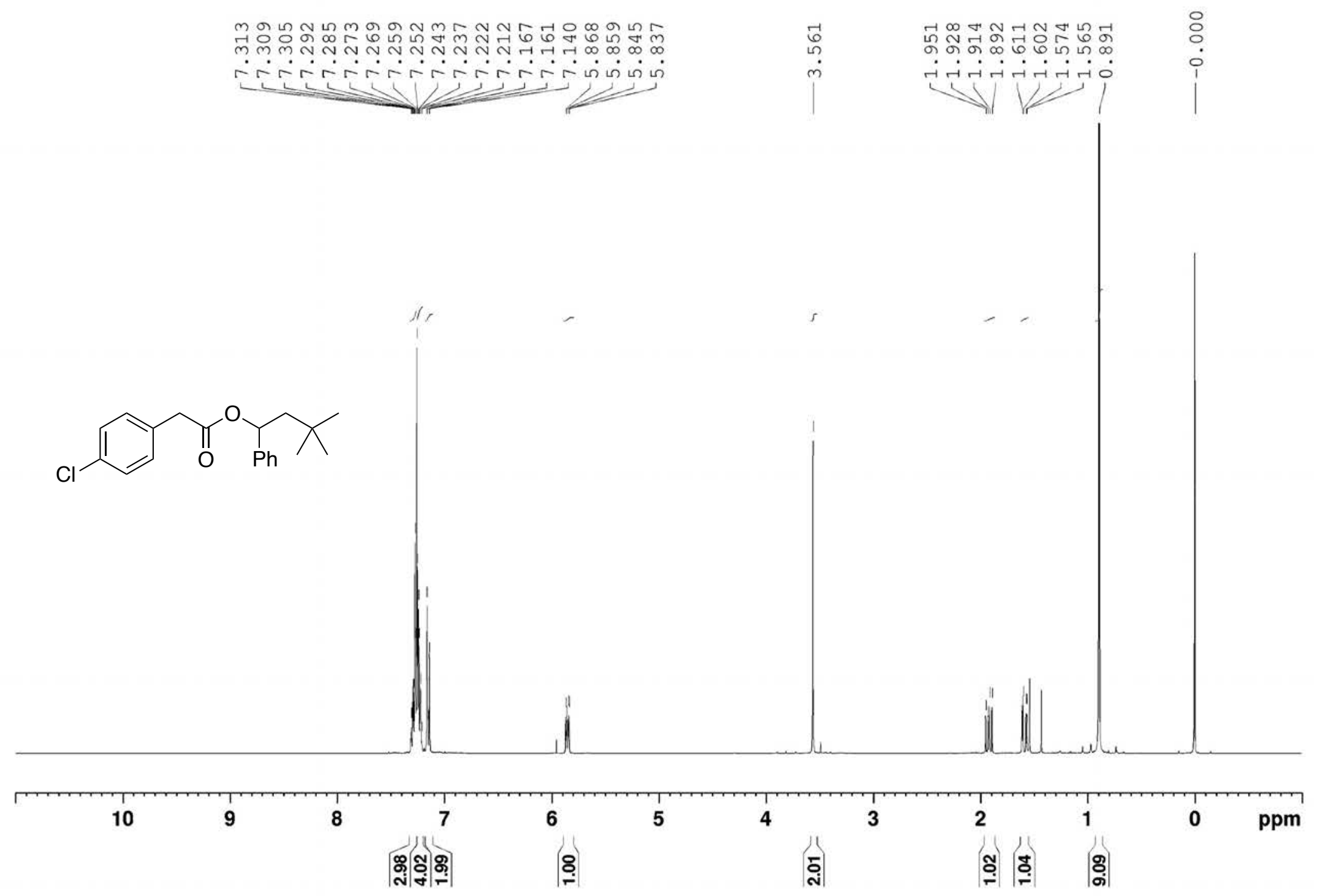

${ }^{1} \mathrm{H}$ NMR spectrum of $11 \mathrm{caa}\left(400 \mathrm{MHz}, \mathrm{CDCl}_{3}\right)$ 


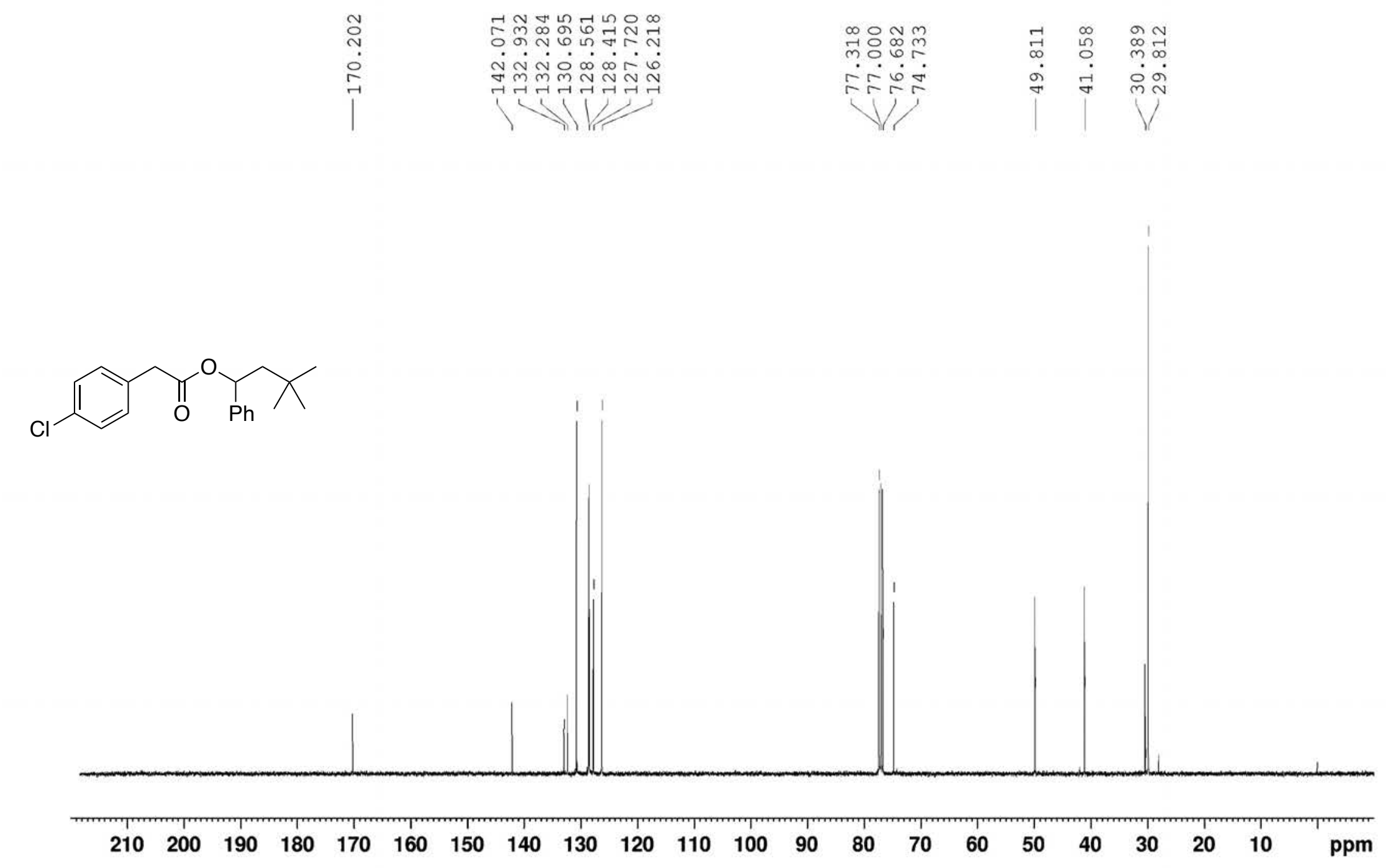

${ }^{13} \mathrm{C}$ NMR spectrum of 11 caa $\left(100.6 \mathrm{MHz}, \mathrm{CDCl}_{3}\right)$ 


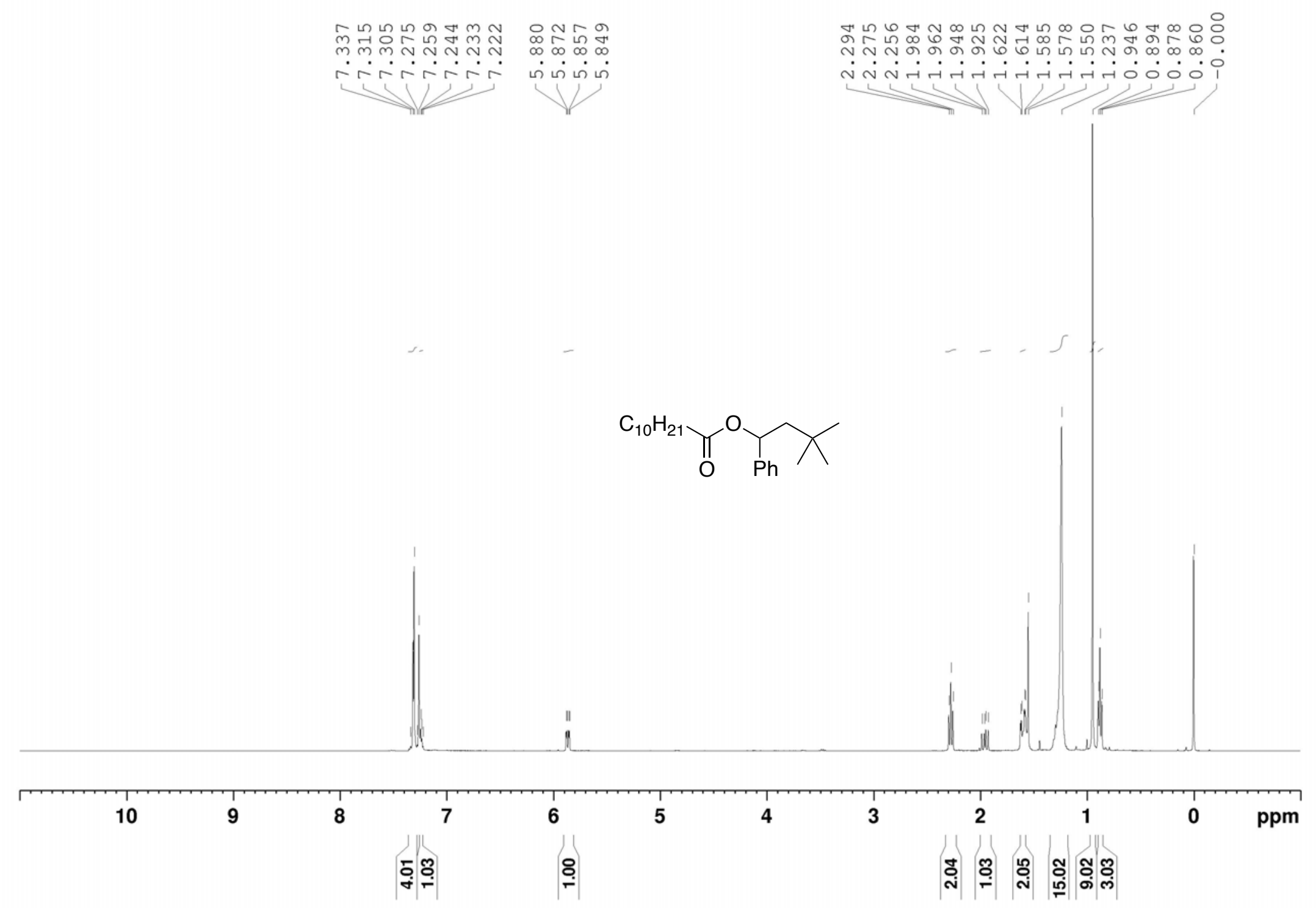

${ }^{1} \mathrm{H}$ NMR spectrum of $\mathbf{1 1 d a a}\left(400 \mathrm{MHz}, \mathrm{CDCl}_{3}\right.$ ) 


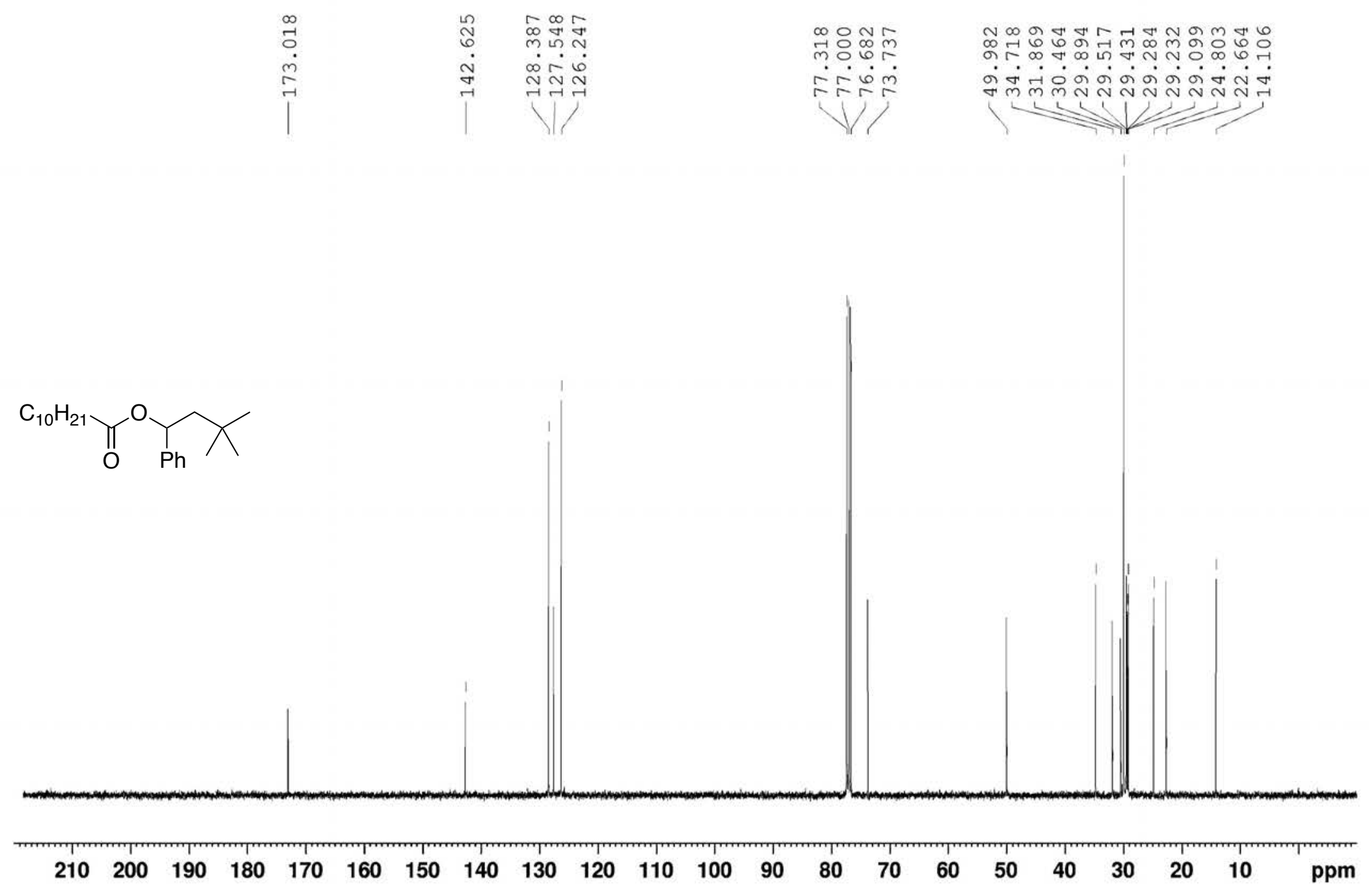

${ }^{13} \mathrm{C}$ NMR spectrum of $\mathbf{1 1 d a a}\left(100.6 \mathrm{MHz}, \mathrm{CDCl}_{3}\right)$ 


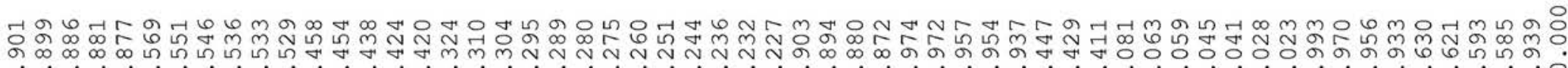

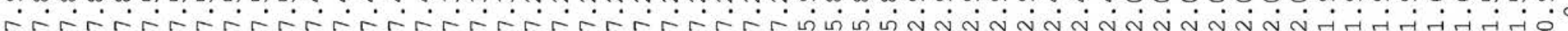

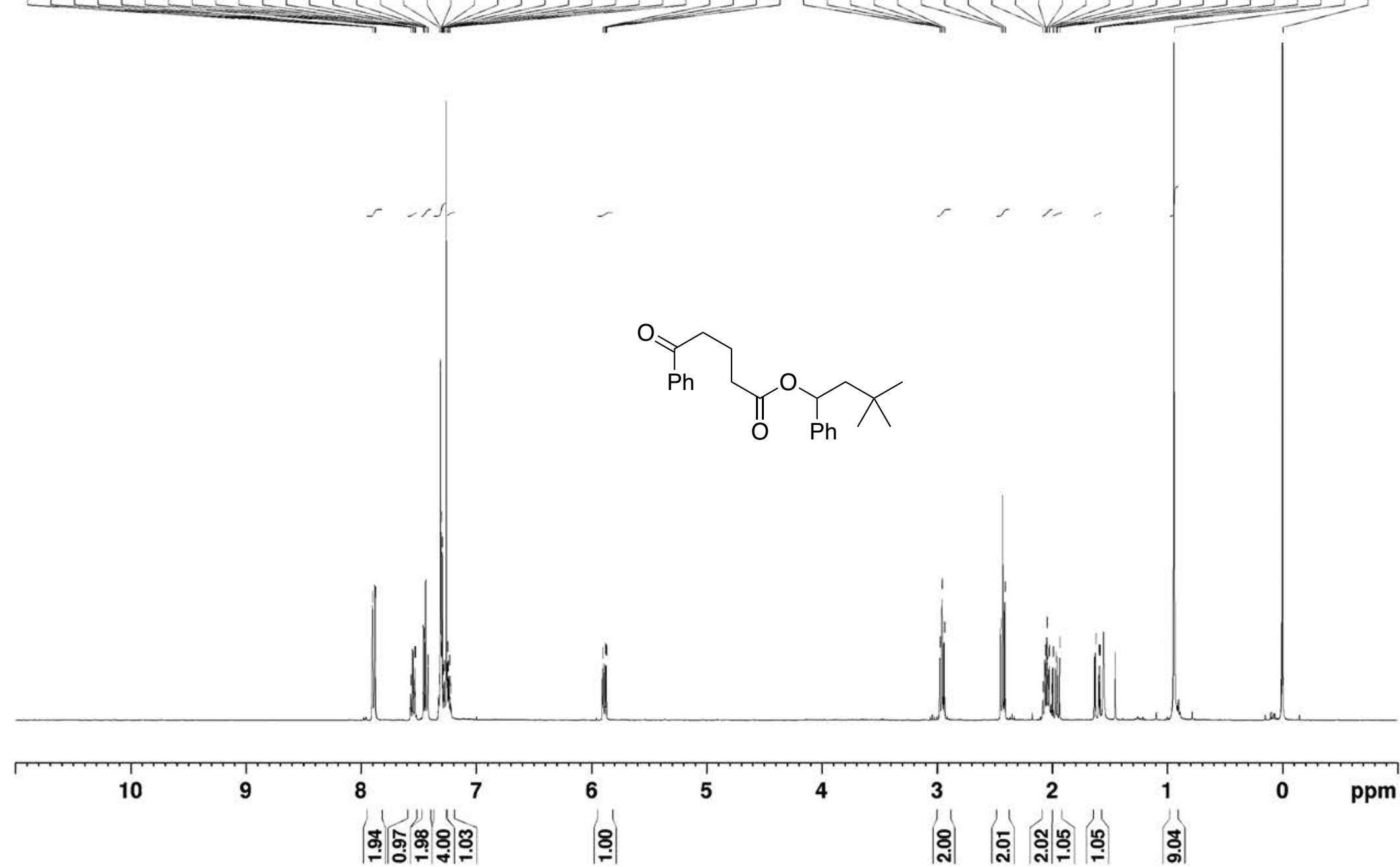

${ }^{1} \mathrm{H}$ NMR spectrum of 11 eaa $\left(400 \mathrm{MHz}, \mathrm{CDCl}_{3}\right)$ 


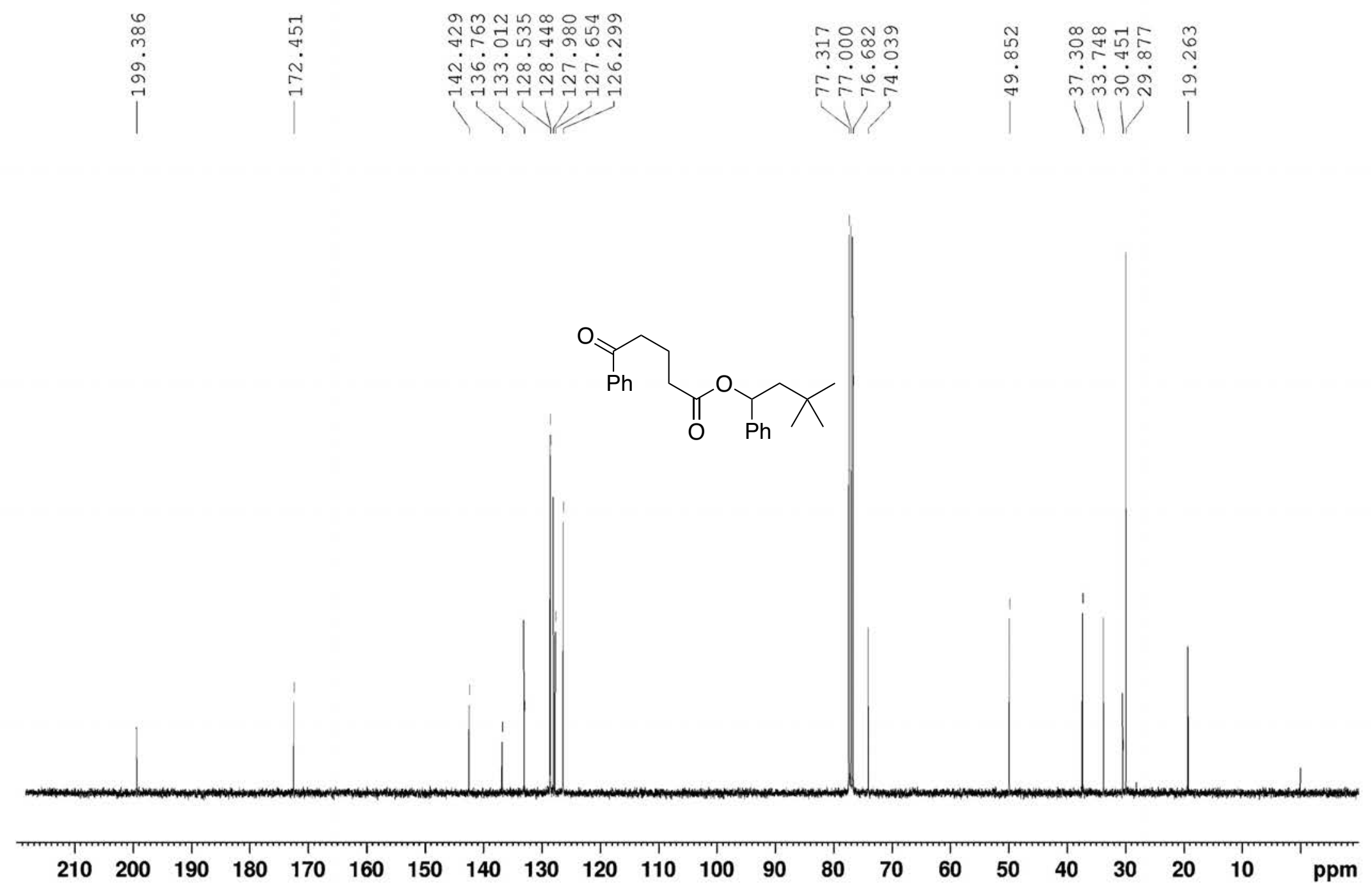

${ }^{13} \mathrm{C}$ NMR spectrum of 11 eaa $\left(100.6 \mathrm{MHz}, \mathrm{CDCl}_{3}\right)$ 


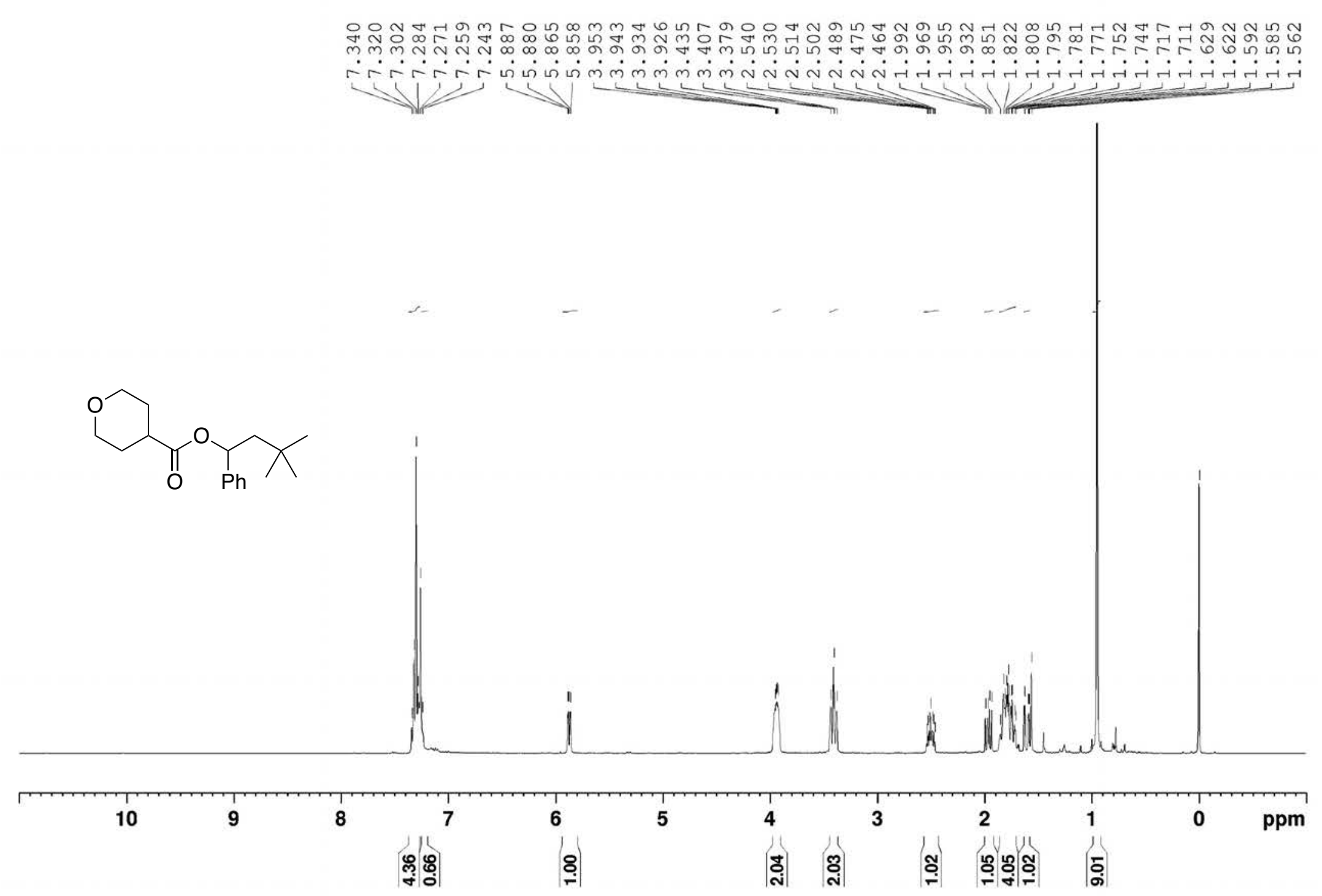

${ }^{1} \mathrm{H}$ NMR spectrum of 11 faa $\left(400 \mathrm{MHz}, \mathrm{CDCl}_{3}\right)$ 


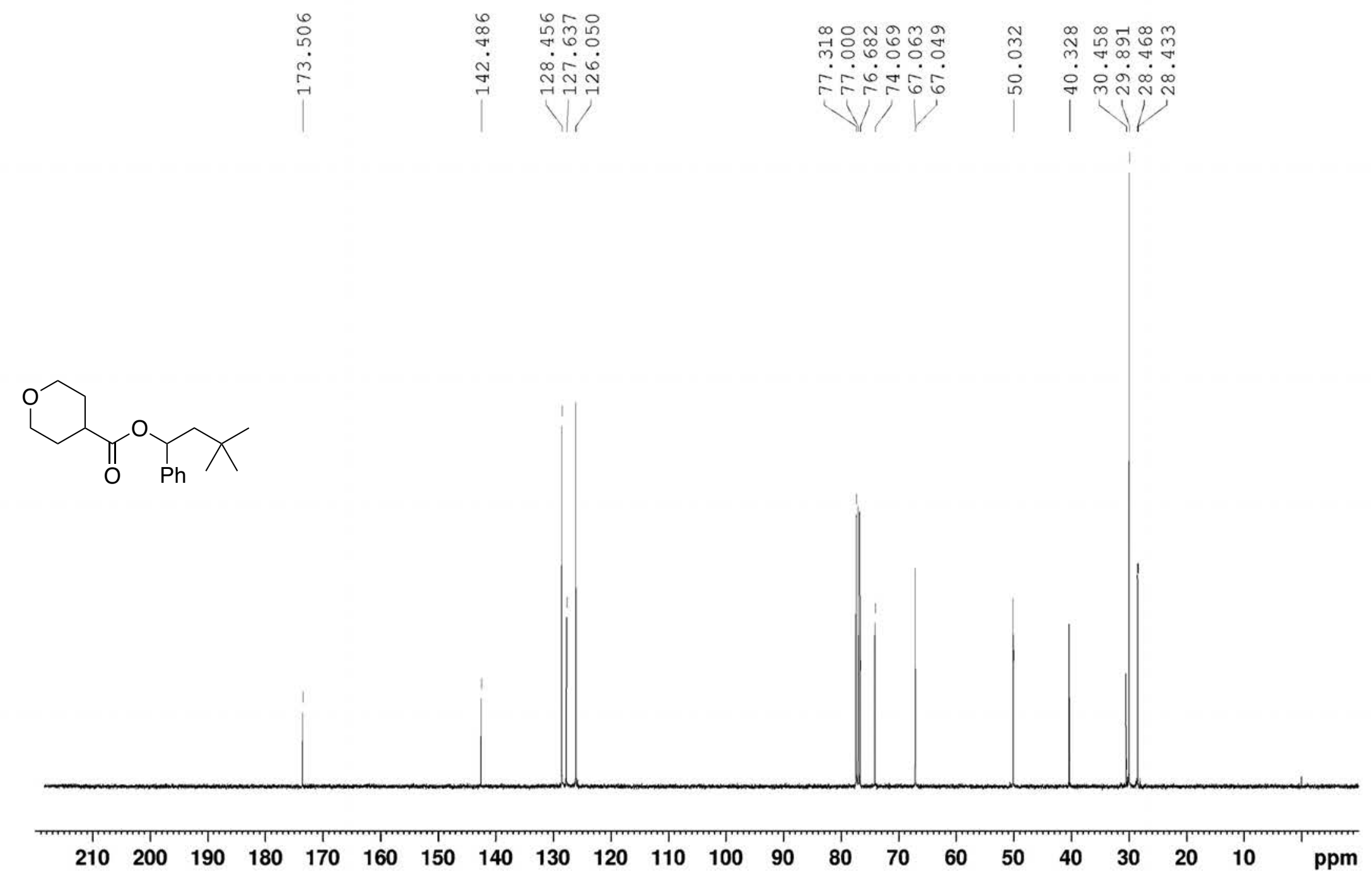

${ }^{13} \mathrm{C}$ NMR spectrum of $\mathbf{1 1}$ faa $\left(100.6 \mathrm{MHz}, \mathrm{CDCl}_{3}\right)$ 


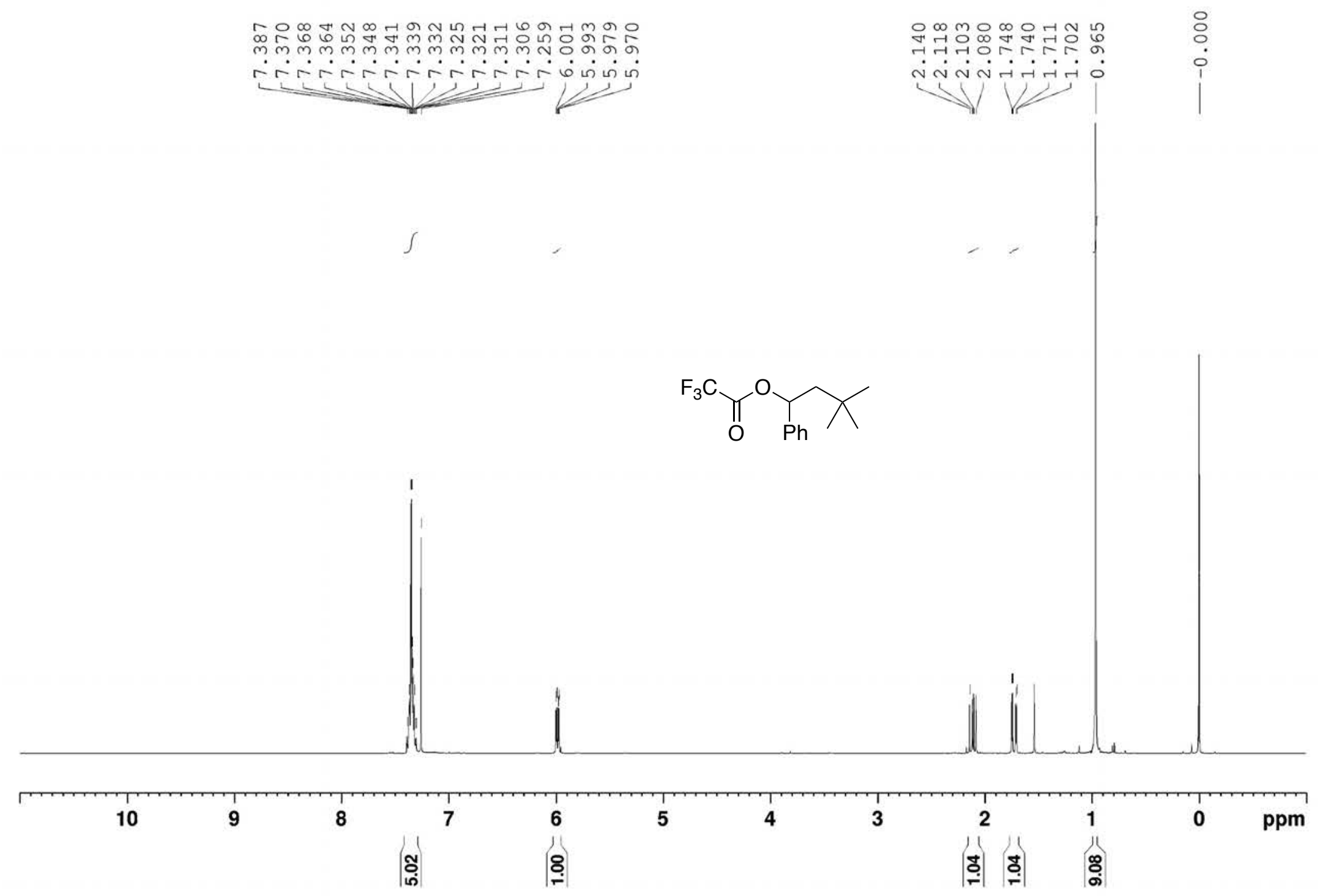

${ }^{1} \mathrm{H}$ NMR spectrum of 11 gaa (400 MHz, $\mathrm{CDCl}_{3}$ ) 


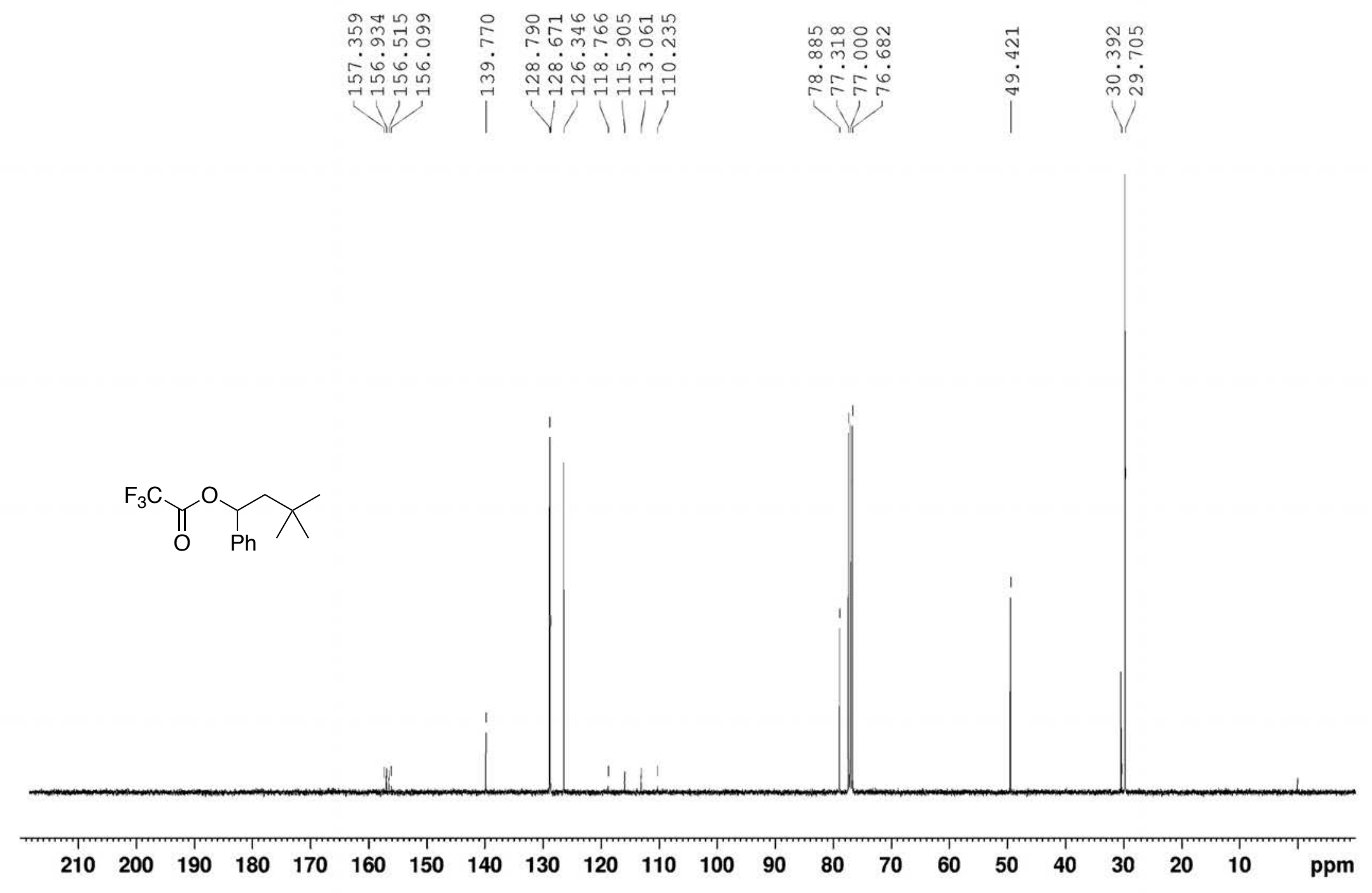

${ }^{13} \mathrm{C}$ NMR spectrum of 11 gaa $\left(100.6 \mathrm{MHz}, \mathrm{CDCl}_{3}\right)$ 


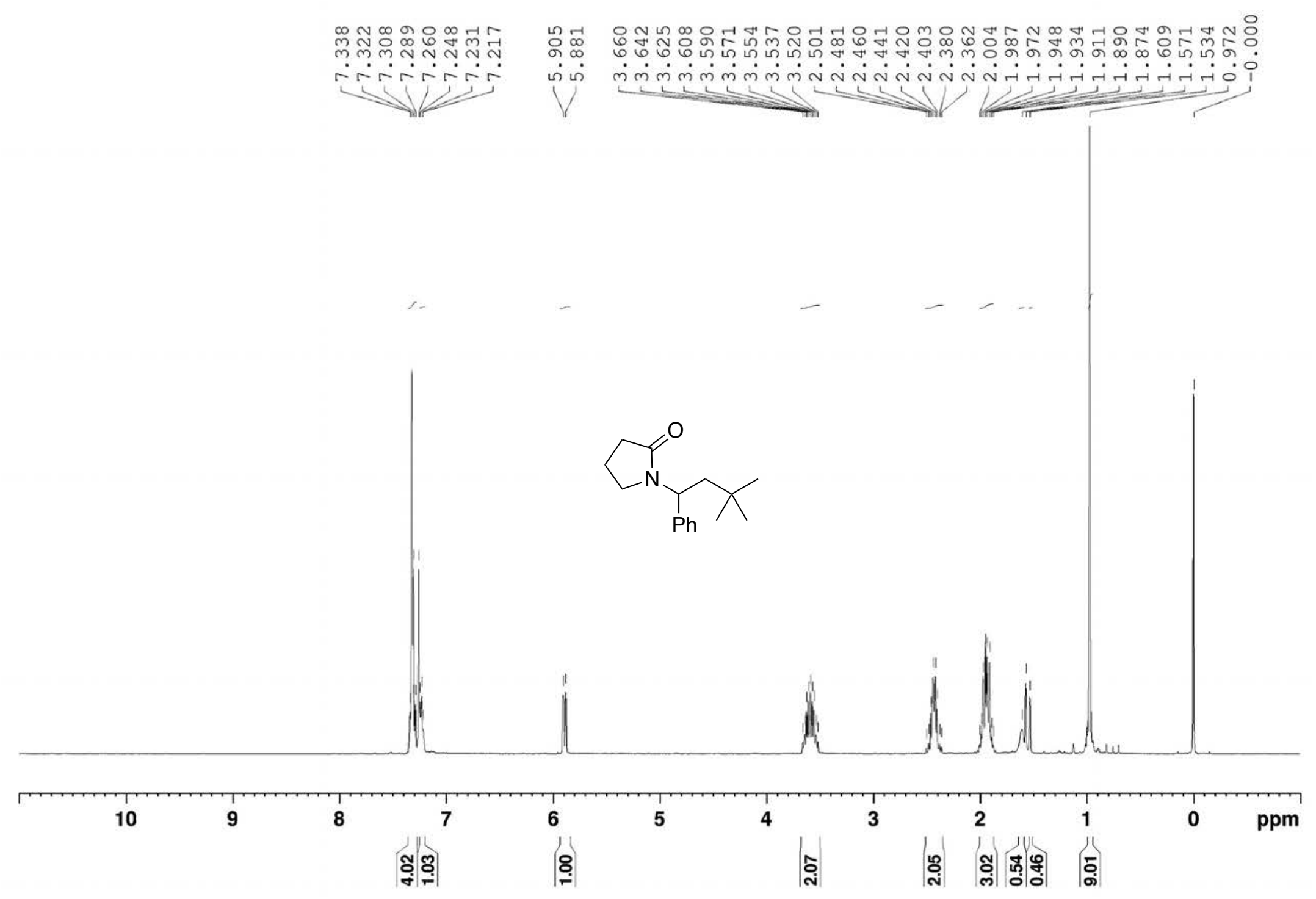

${ }^{1} \mathrm{H}$ NMR spectrum of $\mathbf{1 2 a a a}\left(400 \mathrm{MHz}, \mathrm{CDCl}_{3}\right.$ ) 


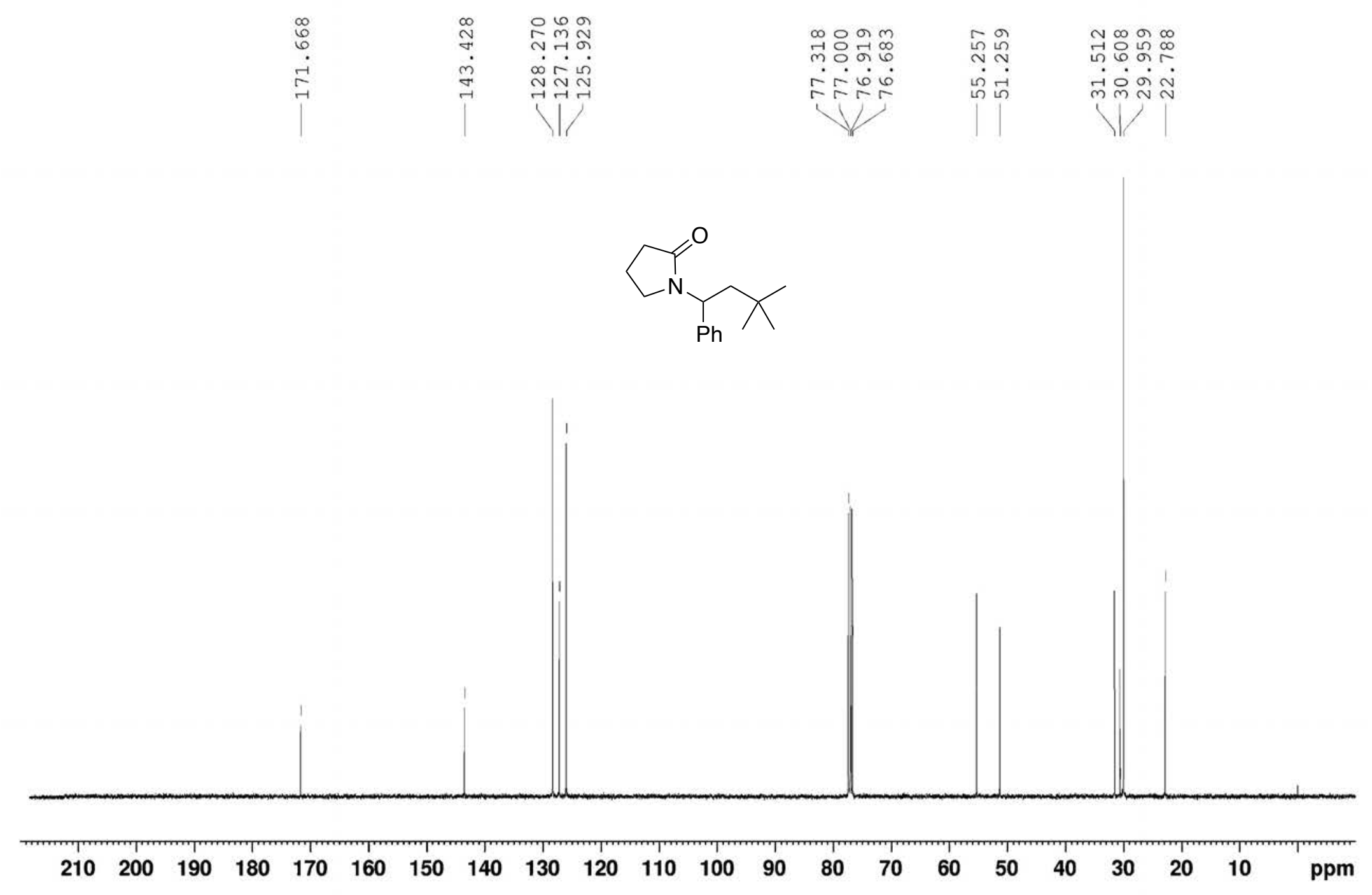

${ }^{13} \mathrm{C}$ NMR spectrum of 12aaa $\left(100.6 \mathrm{MHz}, \mathrm{CDCl}_{3}\right)$ 


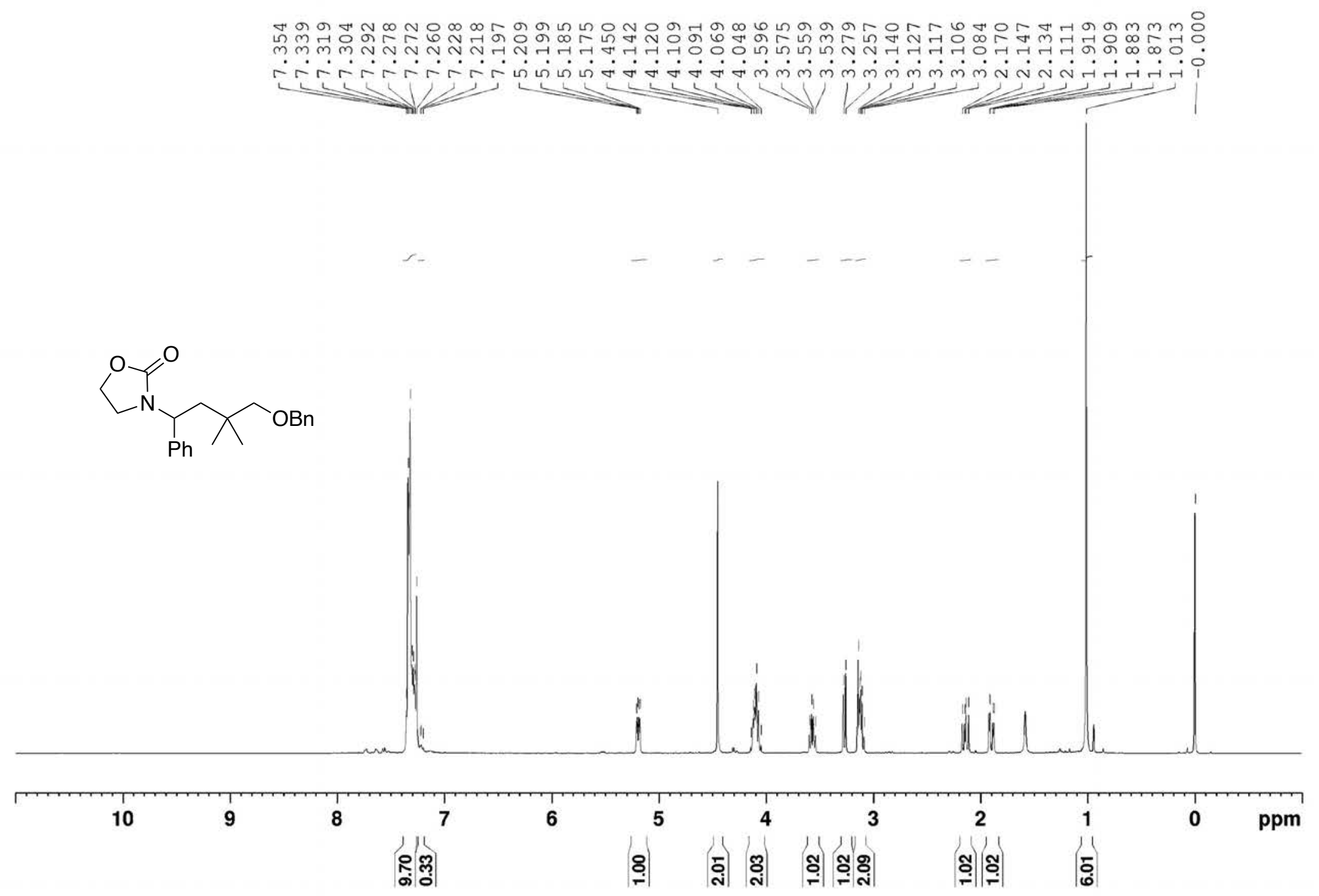

${ }^{1} \mathrm{H}$ NMR spectrum of $\mathbf{1 2 b a c}\left(400 \mathrm{MHz}, \mathrm{CDCl}_{3}\right.$ ) 


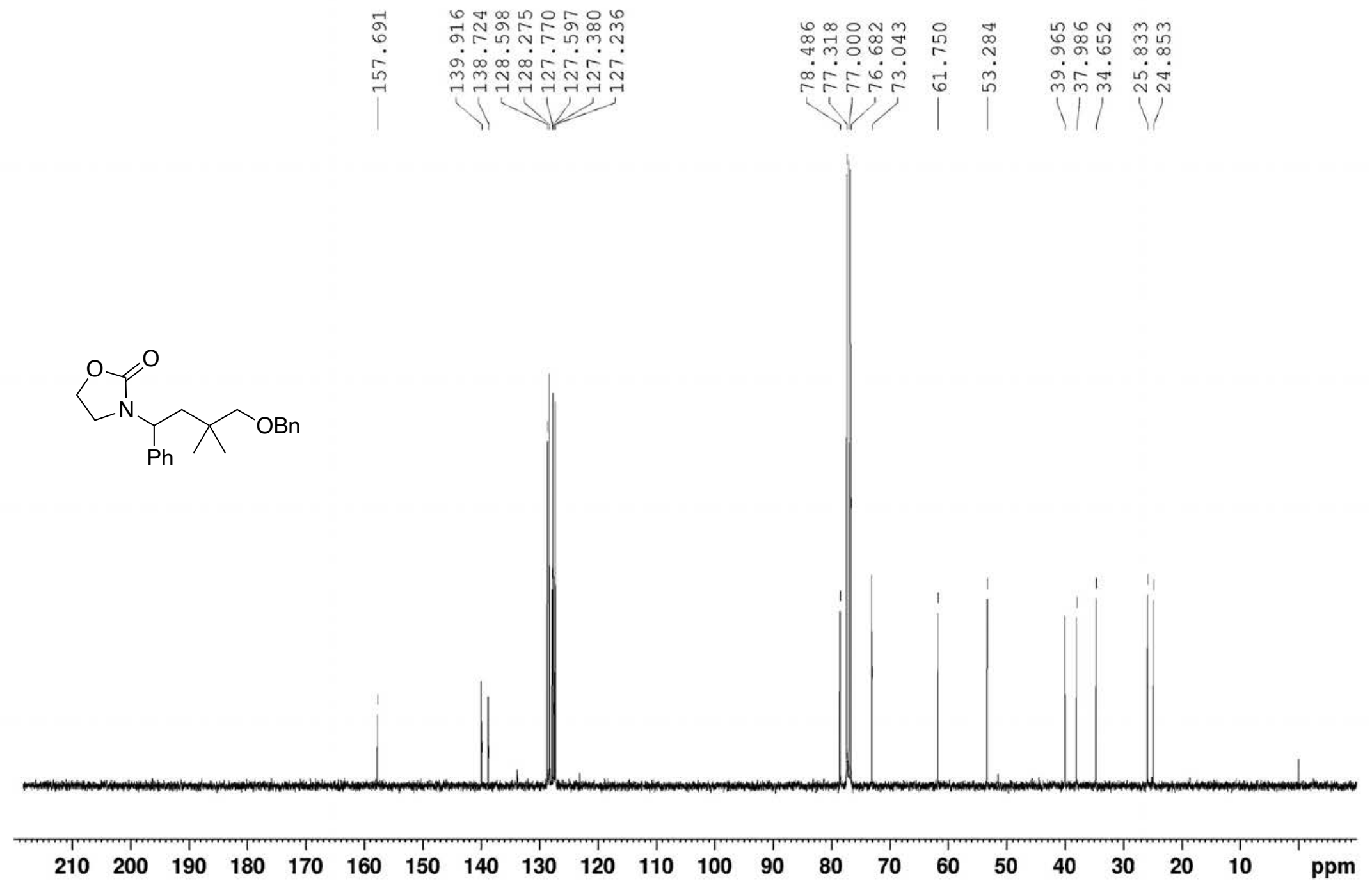

${ }^{13} \mathrm{C}$ NMR spectrum of $\mathbf{1 2 b a c}\left(100.6 \mathrm{MHz}, \mathrm{CDCl}_{3}\right)$ 


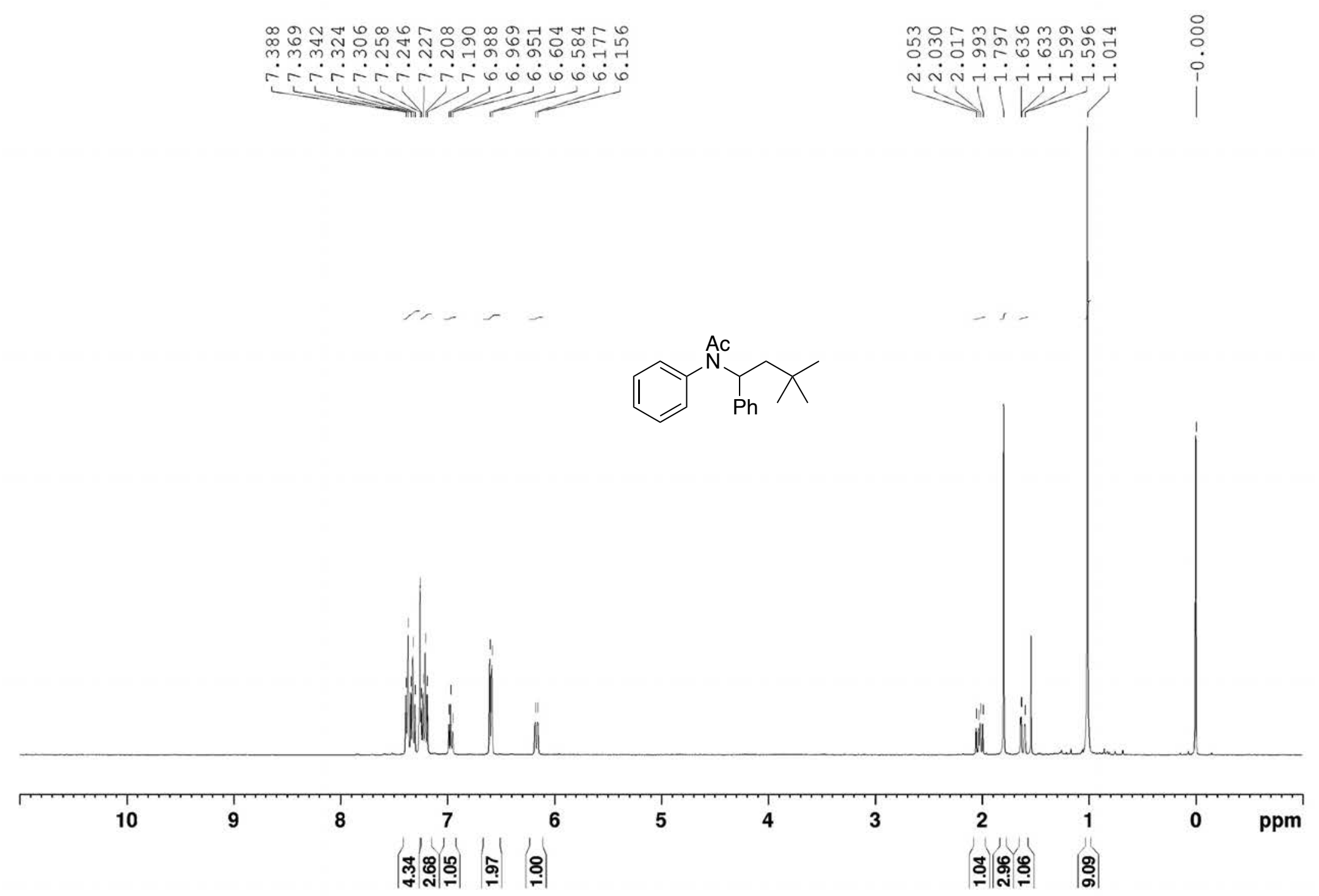

${ }^{1} \mathrm{H}$ NMR spectrum of $\mathbf{1 2 c a a}\left(400 \mathrm{MHz}, \mathrm{CDCl}_{3}\right.$ ) 


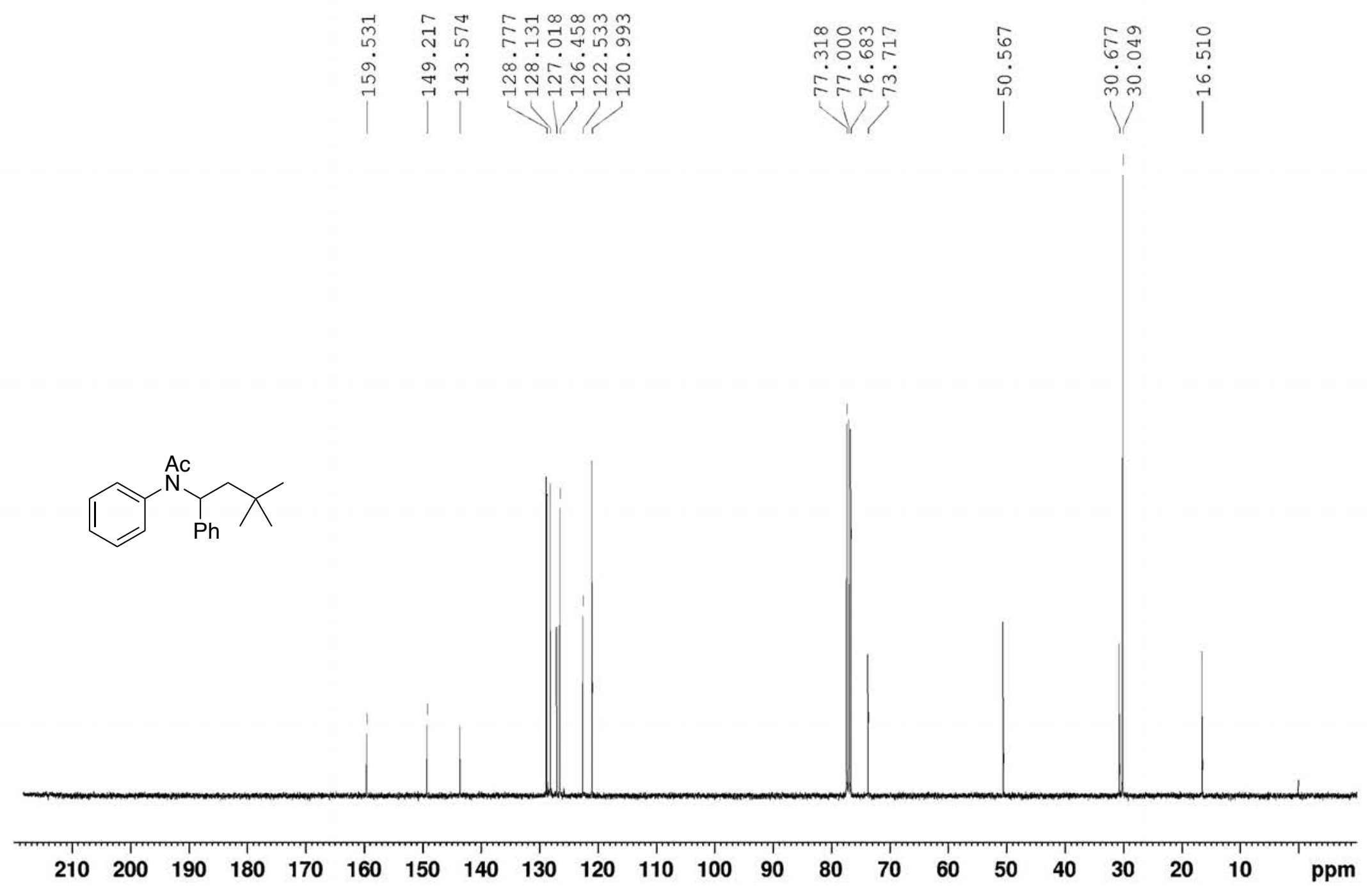

${ }^{13} \mathrm{C}$ NMR spectrum of $\mathbf{1 2}$ caa $\left(100.6 \mathrm{MHz}, \mathrm{CDCl}_{3}\right)$ 


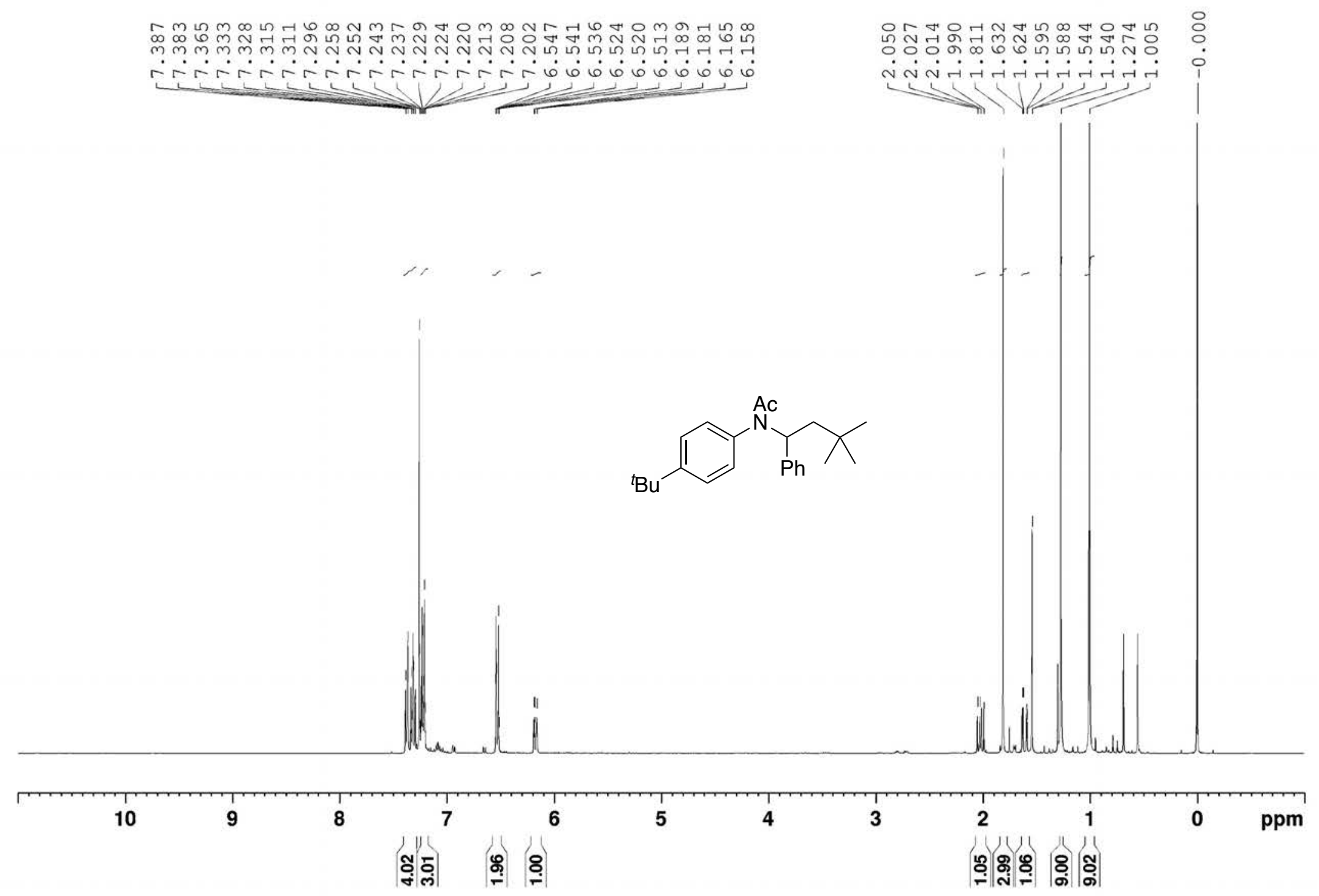

${ }^{1} \mathrm{H}$ NMR spectrum of $\mathbf{1 2 d a a}\left(400 \mathrm{MHz}, \mathrm{CDCl}_{3}\right.$ ) 


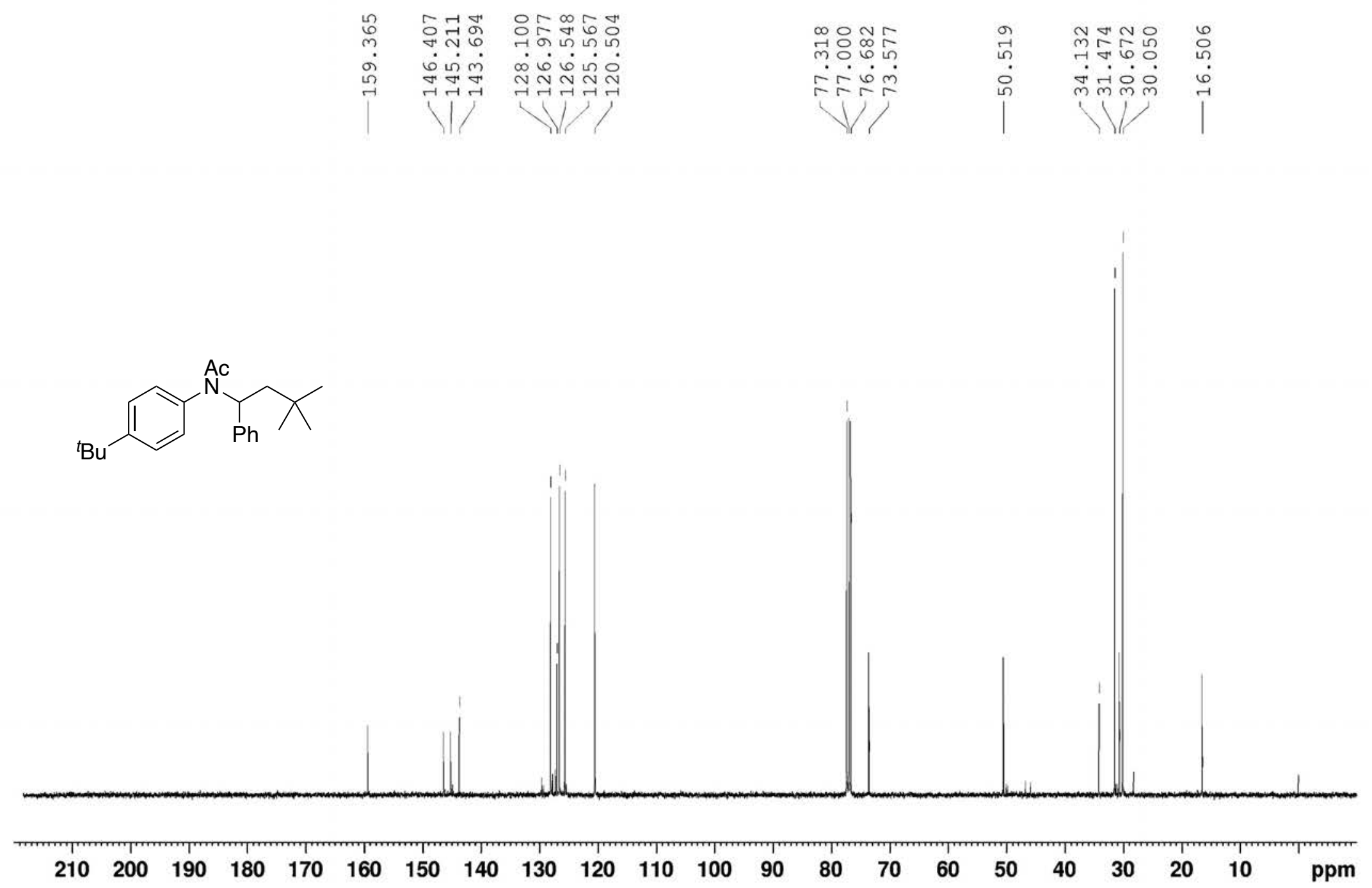

${ }^{13} \mathrm{C}$ NMR spectrum of $\mathbf{1 2 d a a}\left(100.6 \mathrm{MHz}, \mathrm{CDCl}_{3}\right)$ 


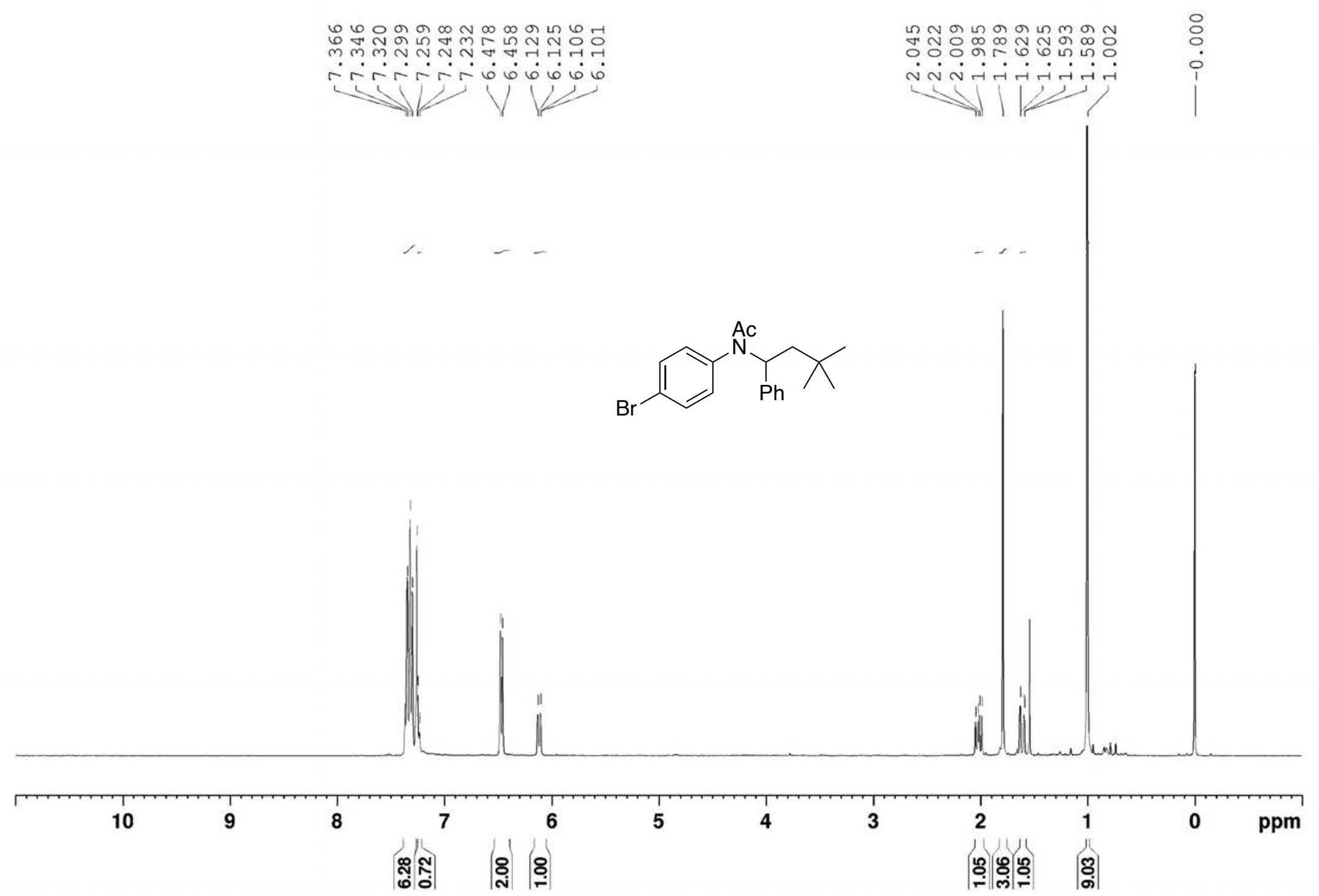

${ }^{1} \mathrm{H}$ NMR spectrum of 12eaa (400 MHz, $\mathrm{CDCl}_{3}$ ) 


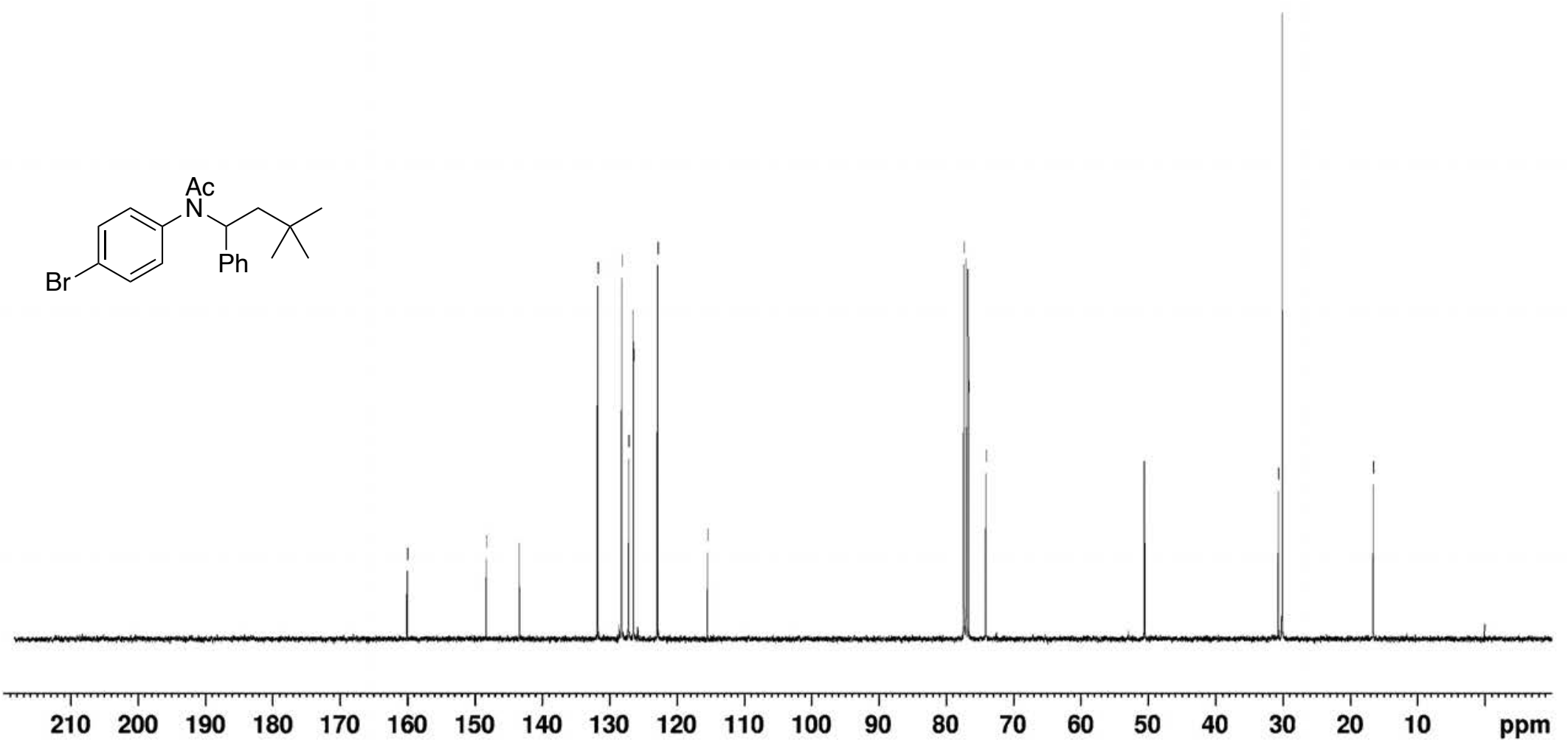

${ }^{13} \mathrm{C}$ NMR spectrum of 12eaa $\left(100.6 \mathrm{MHz}, \mathrm{CDCl}_{3}\right)$ 


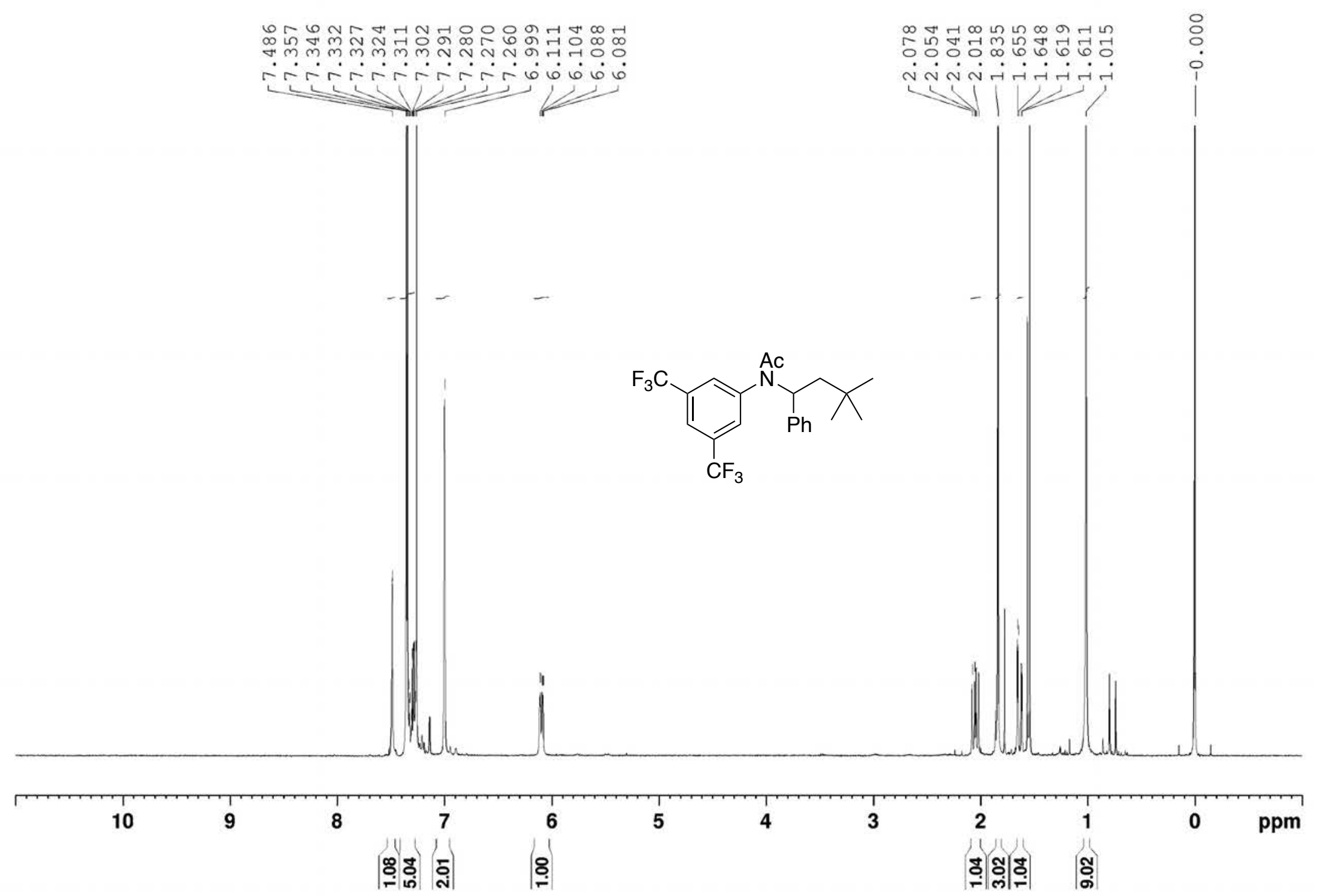

${ }^{1} \mathrm{H}$ NMR spectrum of 12 faa $\left(400 \mathrm{MHz}, \mathrm{CDCl}_{3}\right.$ ) 


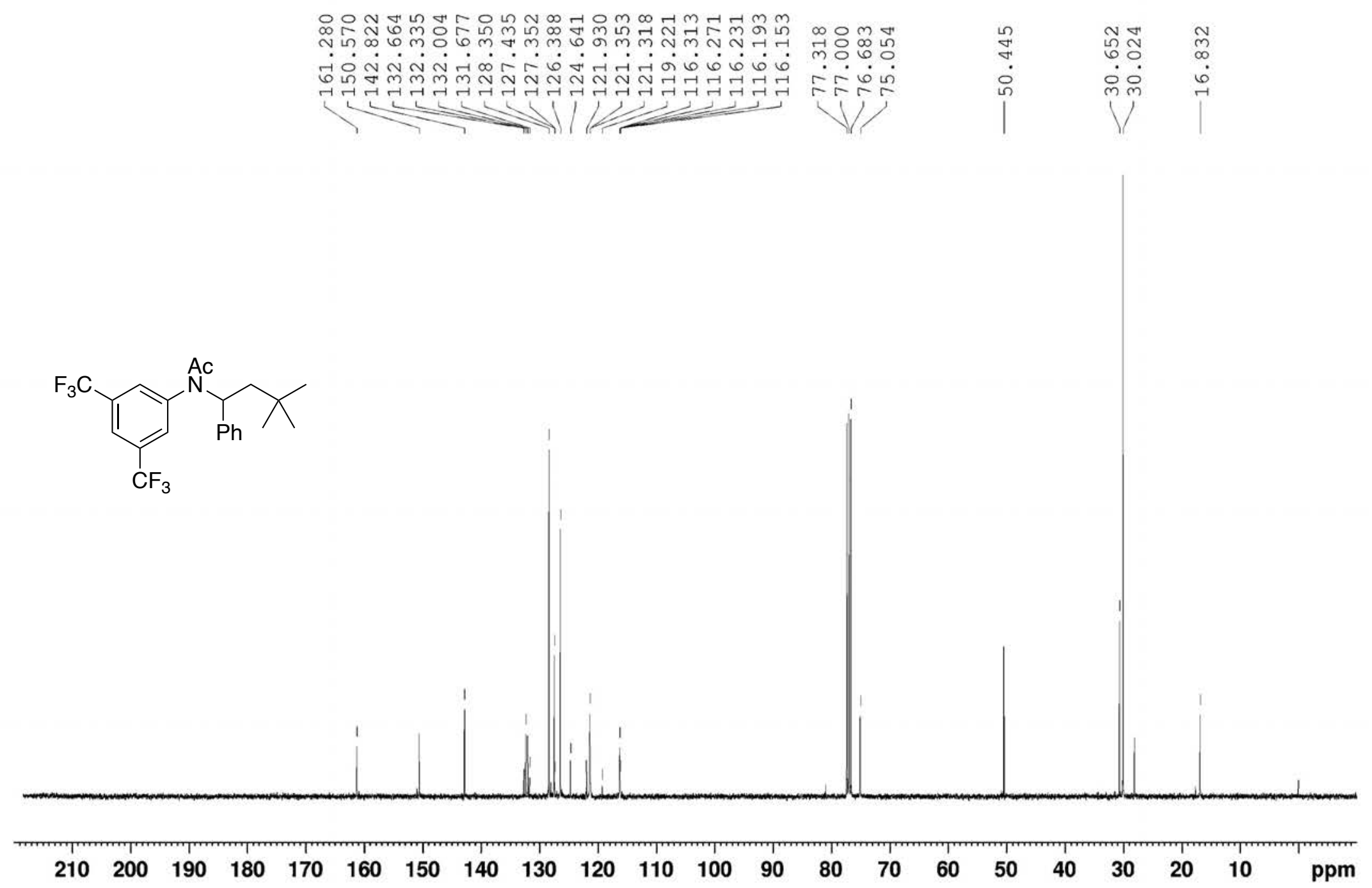

${ }^{13} \mathrm{C}$ NMR spectrum of $\mathbf{1 2 f a a}\left(100.6 \mathrm{MHz}, \mathrm{CDCl}_{3}\right)$ 


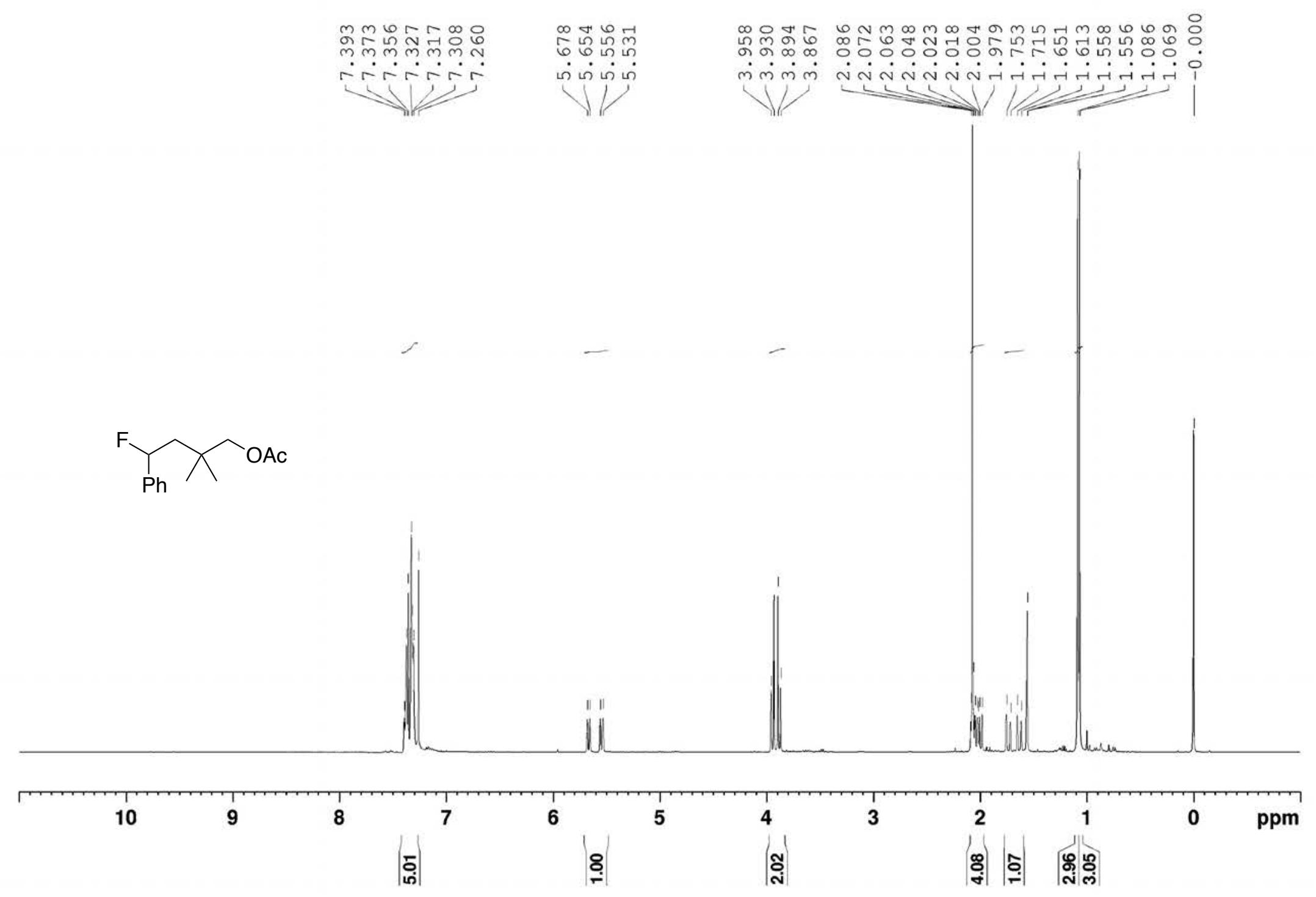

${ }^{1} \mathrm{H}$ NMR spectrum of $\mathbf{1 3 a a b}\left(400 \mathrm{MHz}, \mathrm{CDCl}_{3}\right)$ 


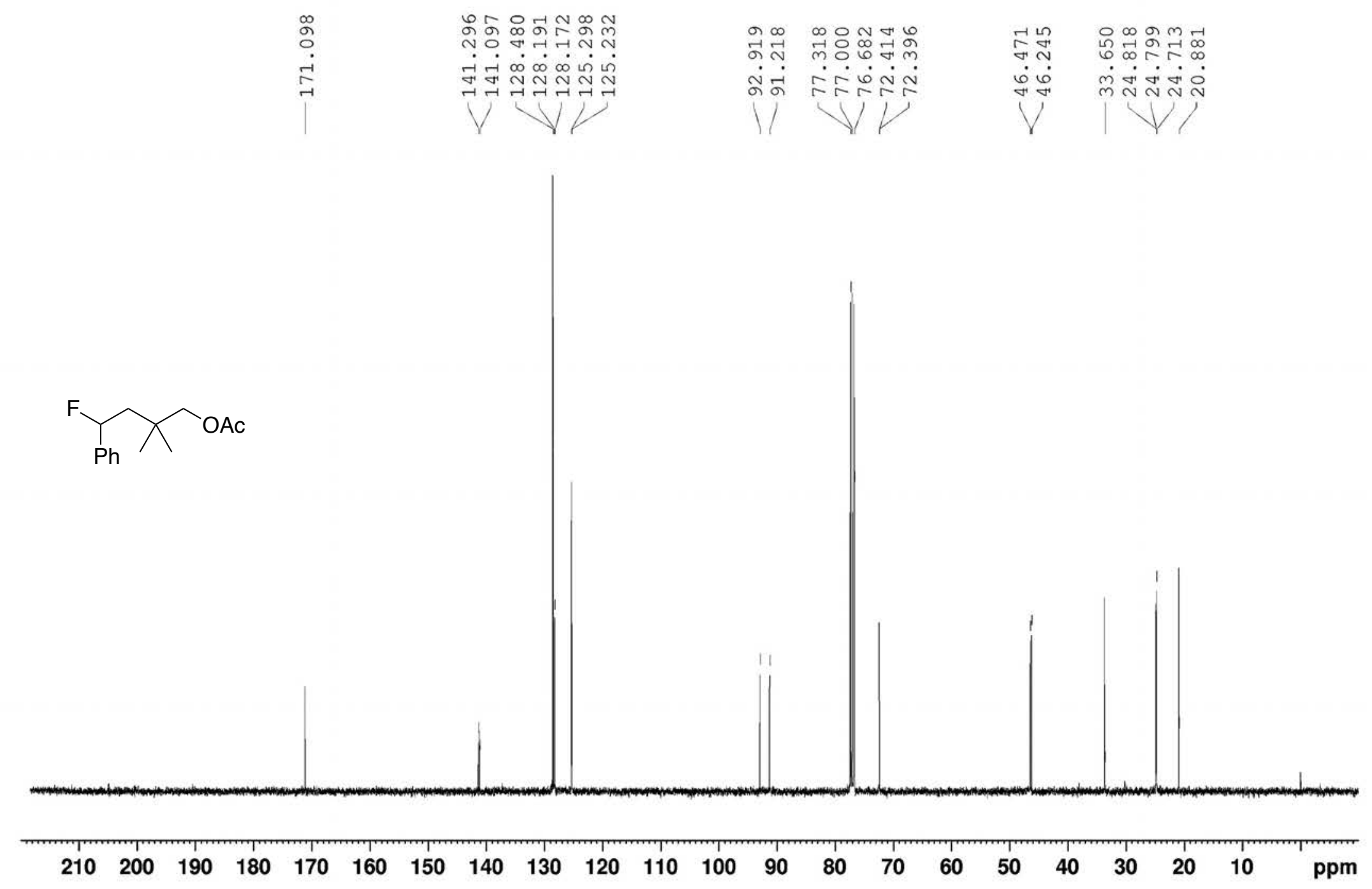

${ }^{13} \mathrm{C}$ NMR spectrum of $\mathbf{1 3 a a b}\left(100.6 \mathrm{MHz}, \mathrm{CDCl}_{3}\right)$ 


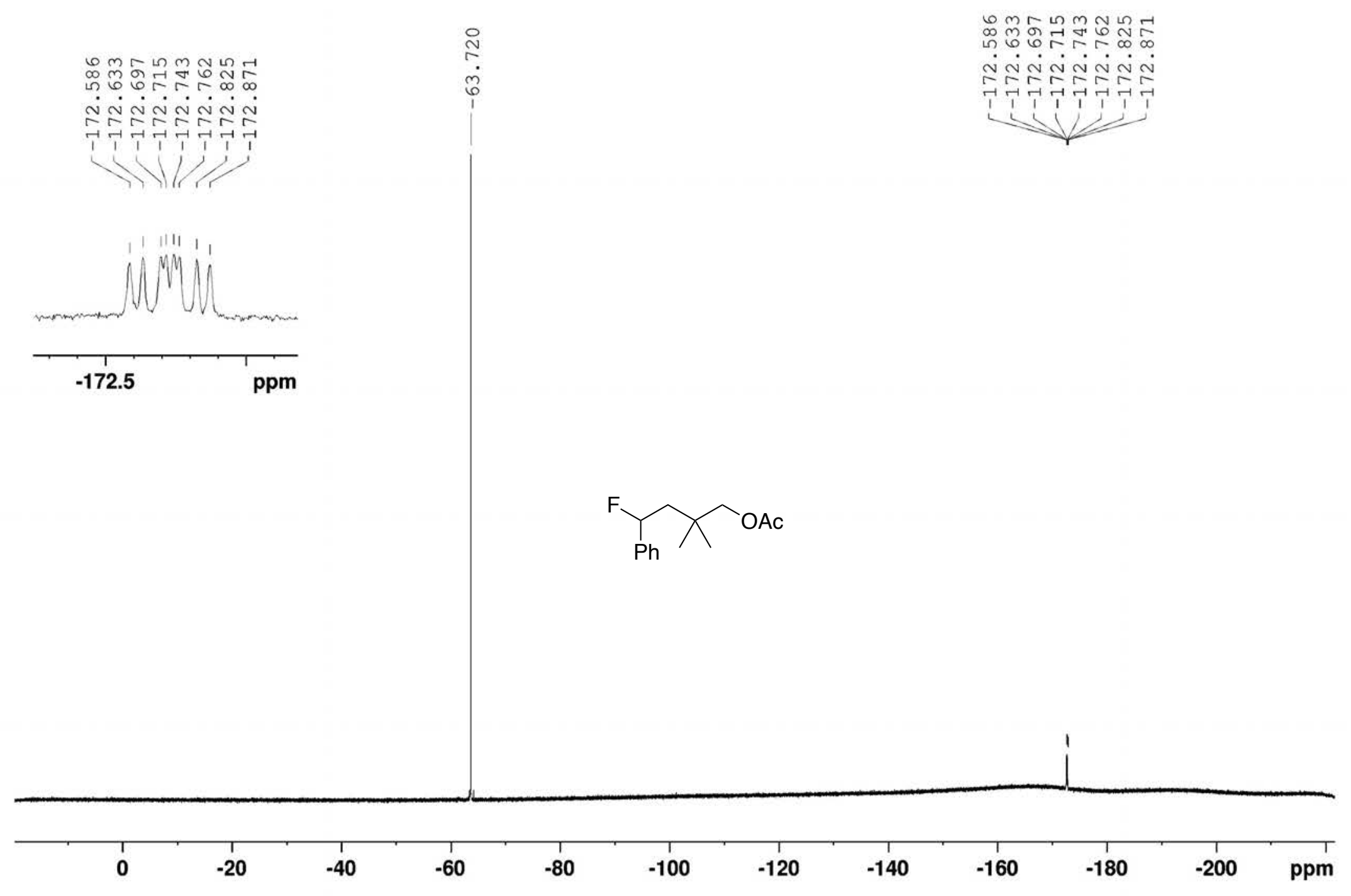

${ }^{19} \mathrm{~F}$ NMR spectrum of 13aab (376.5 MHz, $\mathrm{CDCl}_{3}$ ) 


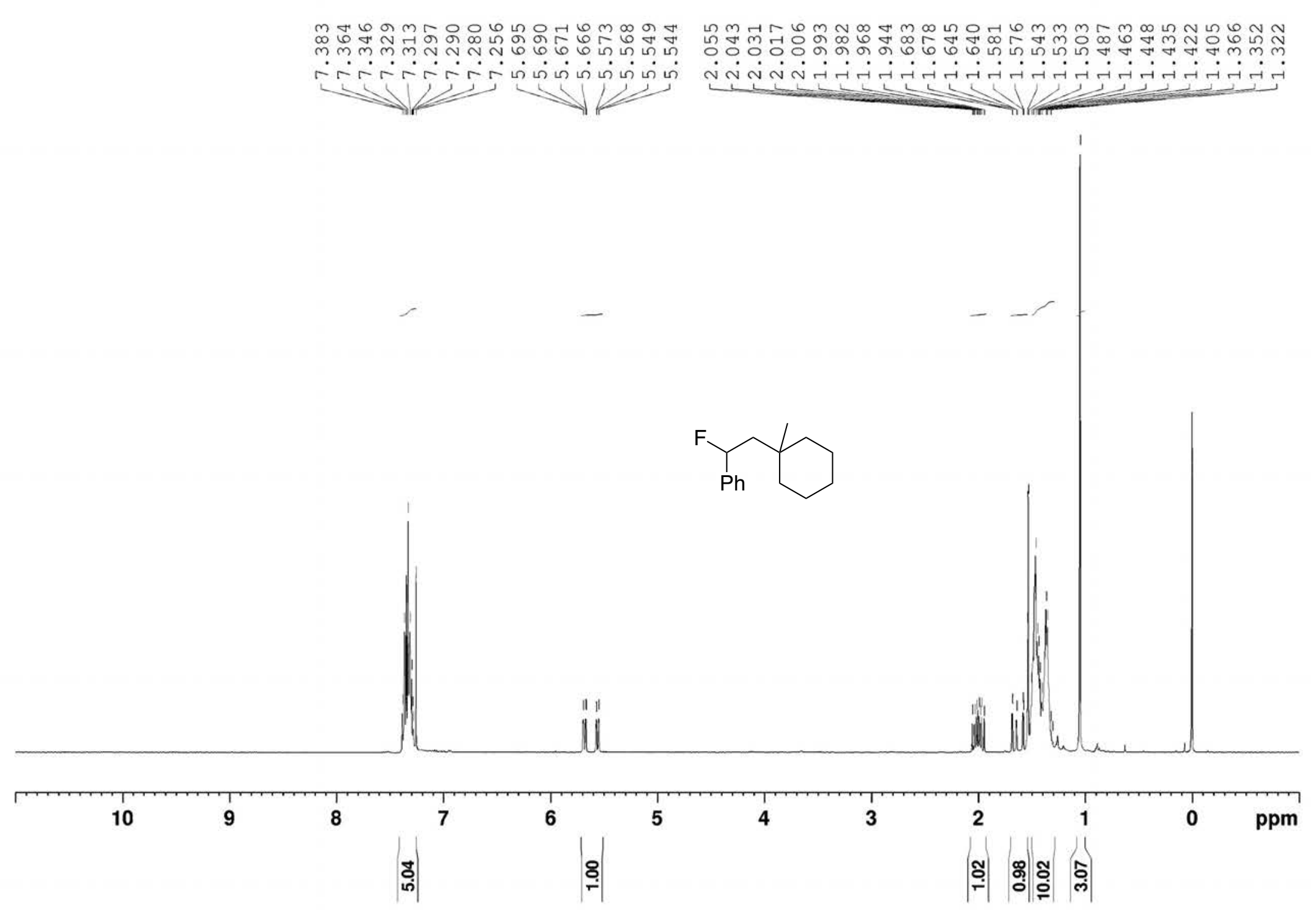

${ }^{1} \mathrm{H}$ NMR spectrum of 13aaf $\left(400 \mathrm{MHz}, \mathrm{CDCl}_{3}\right)$ 


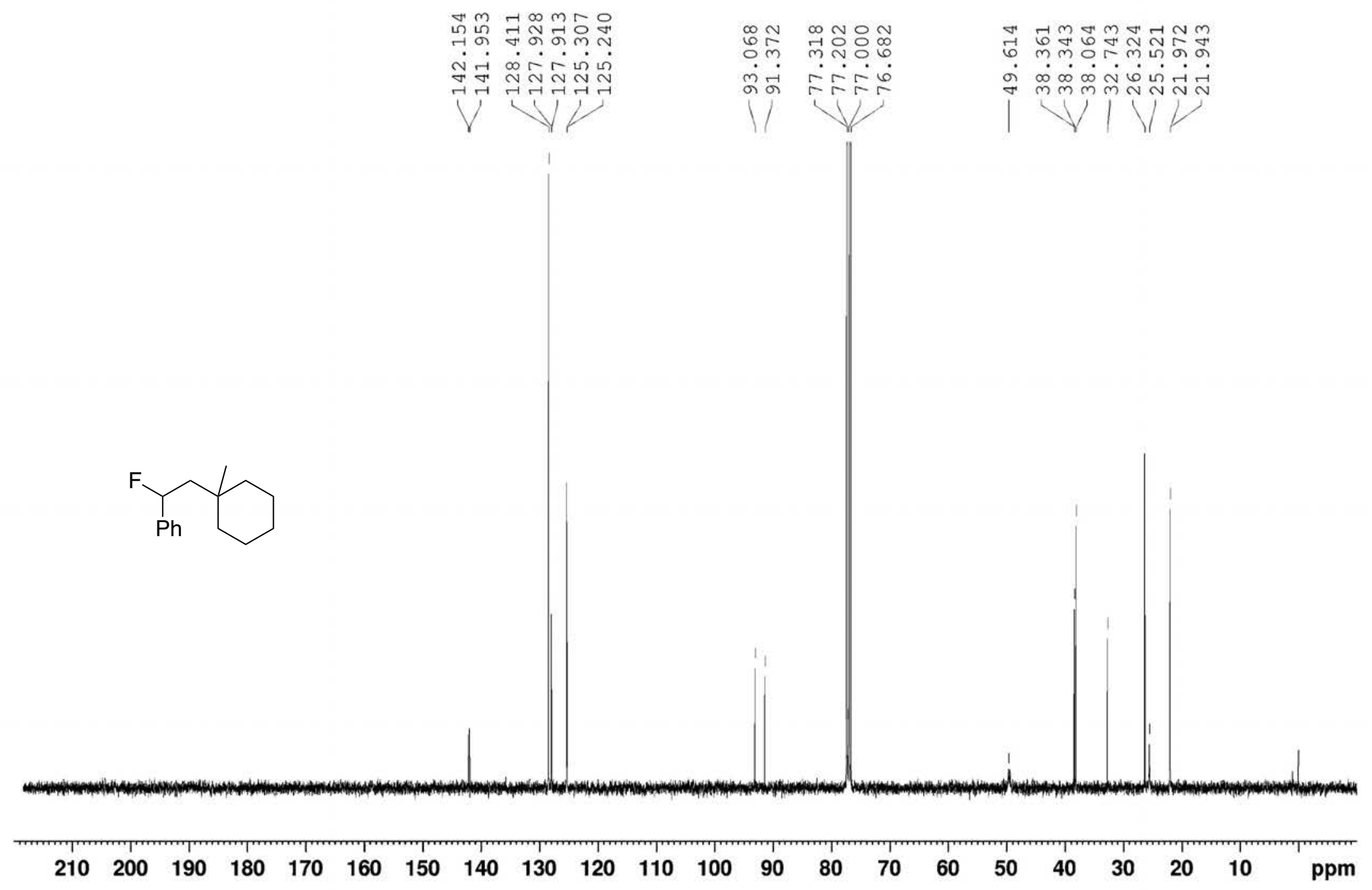

${ }^{13} \mathrm{C}$ NMR spectrum of 13aaf $\left(100.6 \mathrm{MHz}, \mathrm{CDCl}_{3}\right)$ 


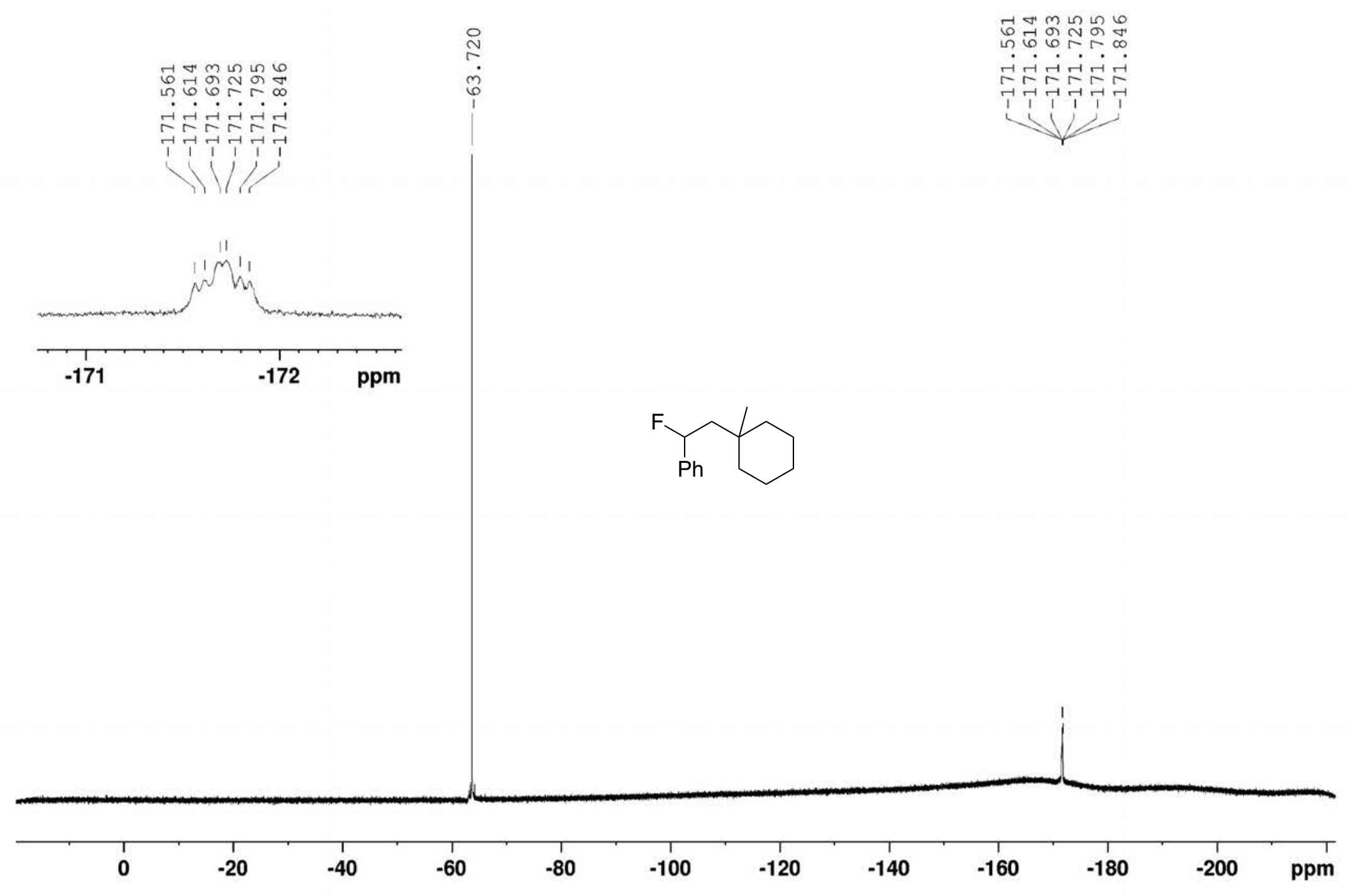

${ }^{19} \mathrm{~F}$ NMR spectrum of $\mathbf{1 3 a a f}\left(376.5 \mathrm{MHz}, \mathrm{CDCl}_{3}\right)$ 


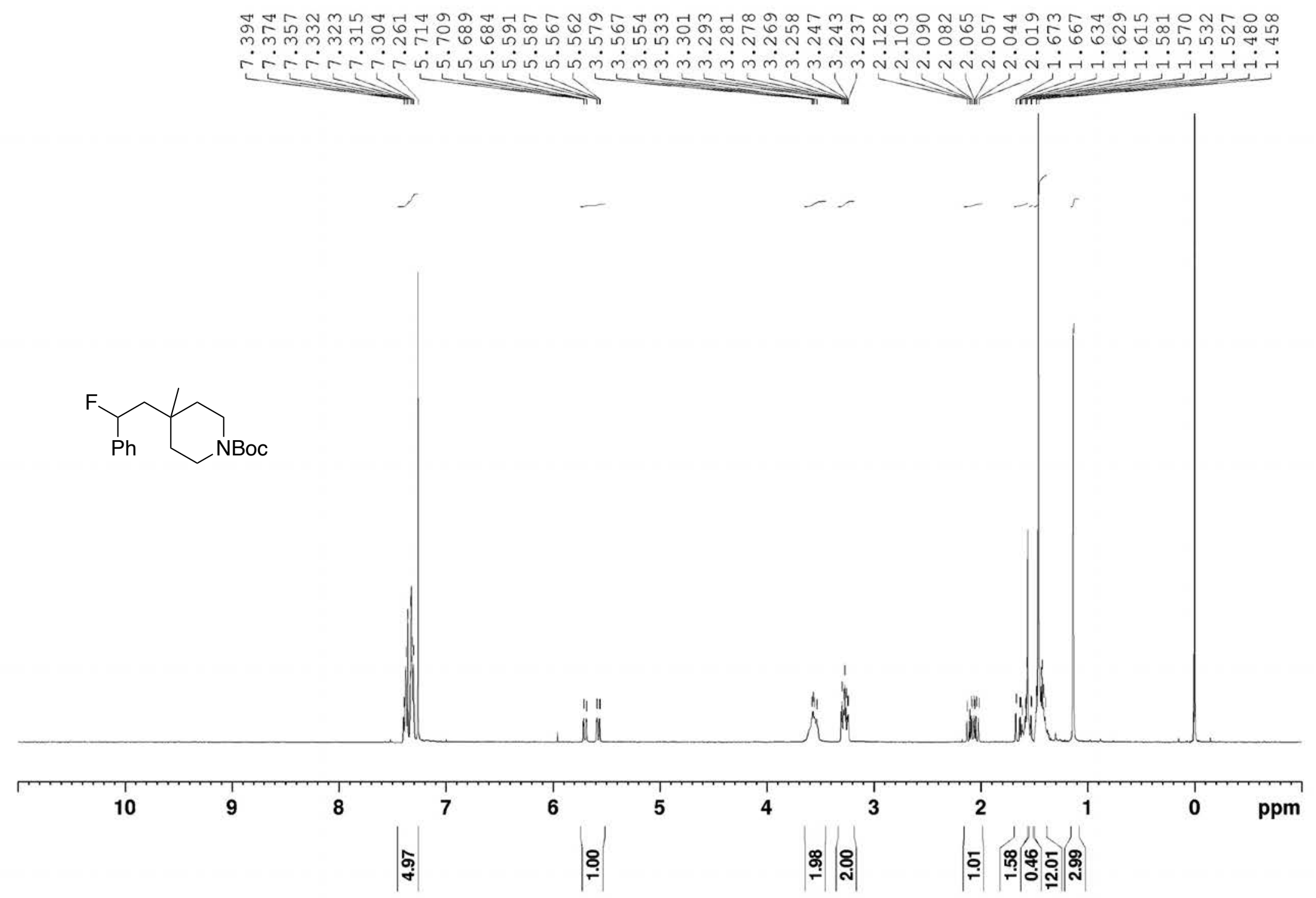

${ }^{1} \mathrm{H}$ NMR spectrum of $13 \mathbf{a a g}$ (400 MHz, $\mathrm{CDCl}_{3}$ ) 


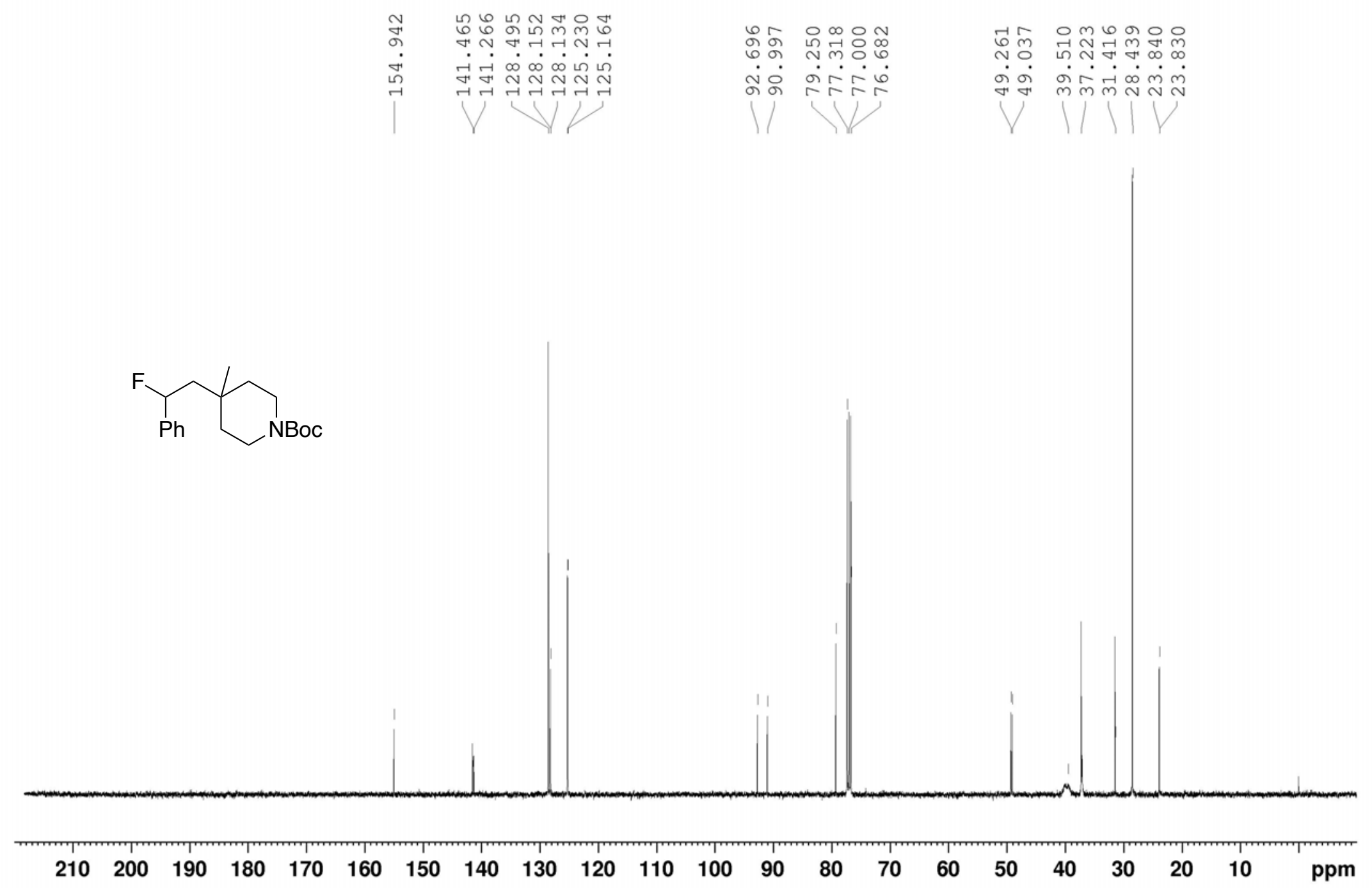

${ }^{13} \mathrm{C}$ NMR spectrum of $13 \mathbf{a a g}\left(100.6 \mathrm{MHz}, \mathrm{CDCl}_{3}\right)$ 


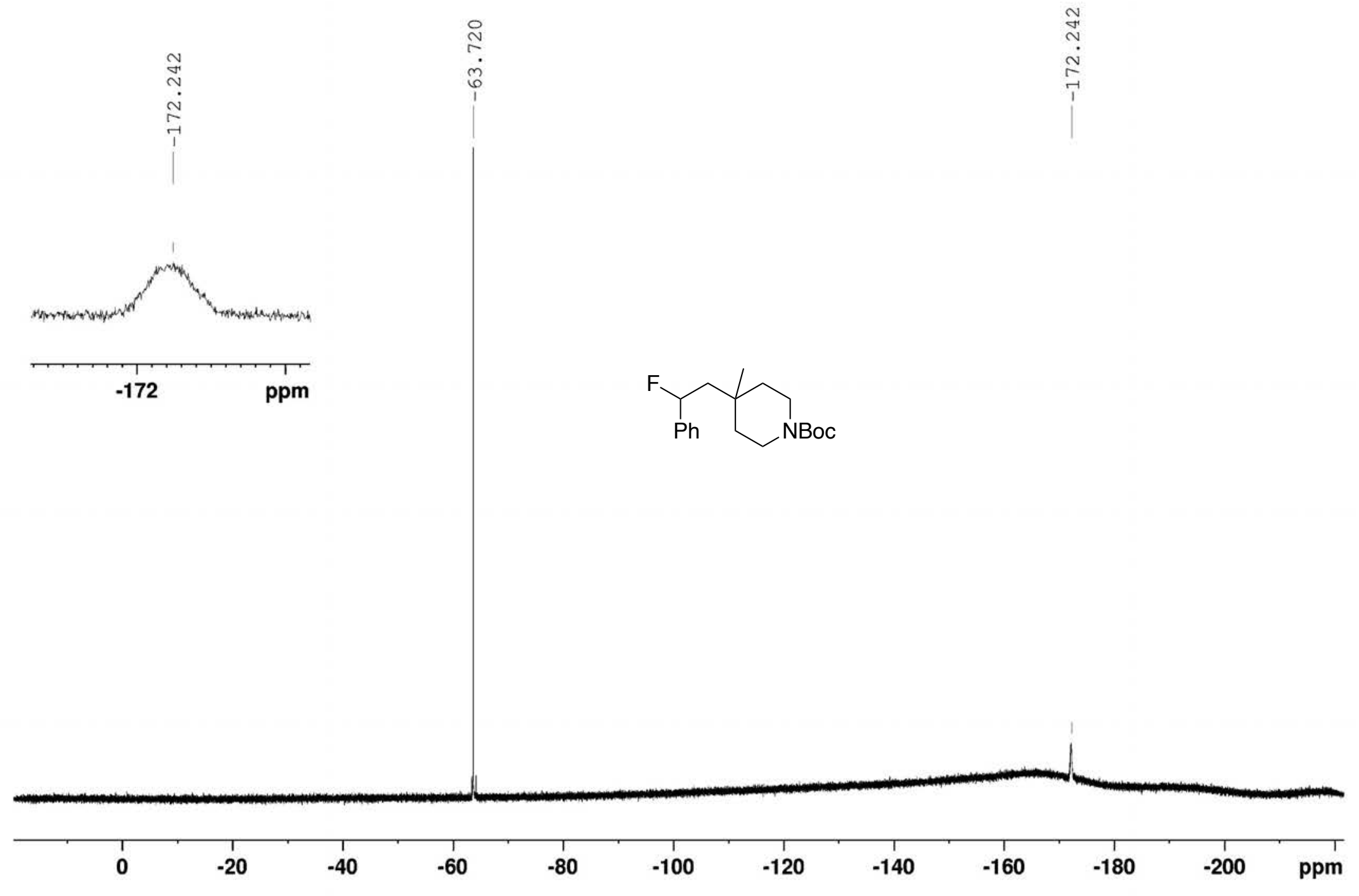

${ }^{19} \mathrm{~F}$ NMR spectrum of $13 \mathrm{aag}\left(376.5 \mathrm{MHz}, \mathrm{CDCl}_{3}\right)$ 

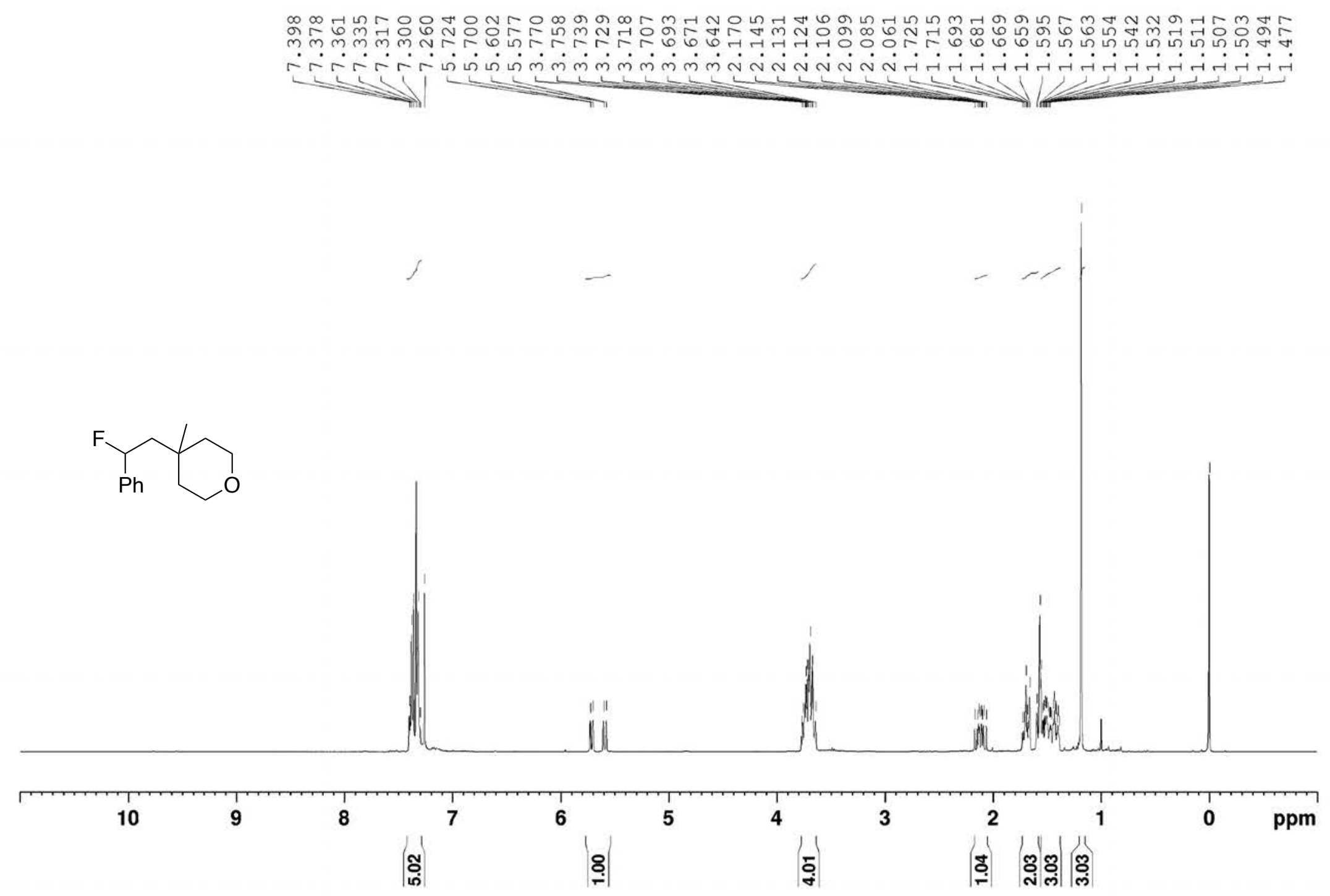

${ }^{1} \mathrm{H}$ NMR spectrum of $\mathbf{1 3 a a h}\left(400 \mathrm{MHz}, \mathrm{CDCl}_{3}\right)$ 


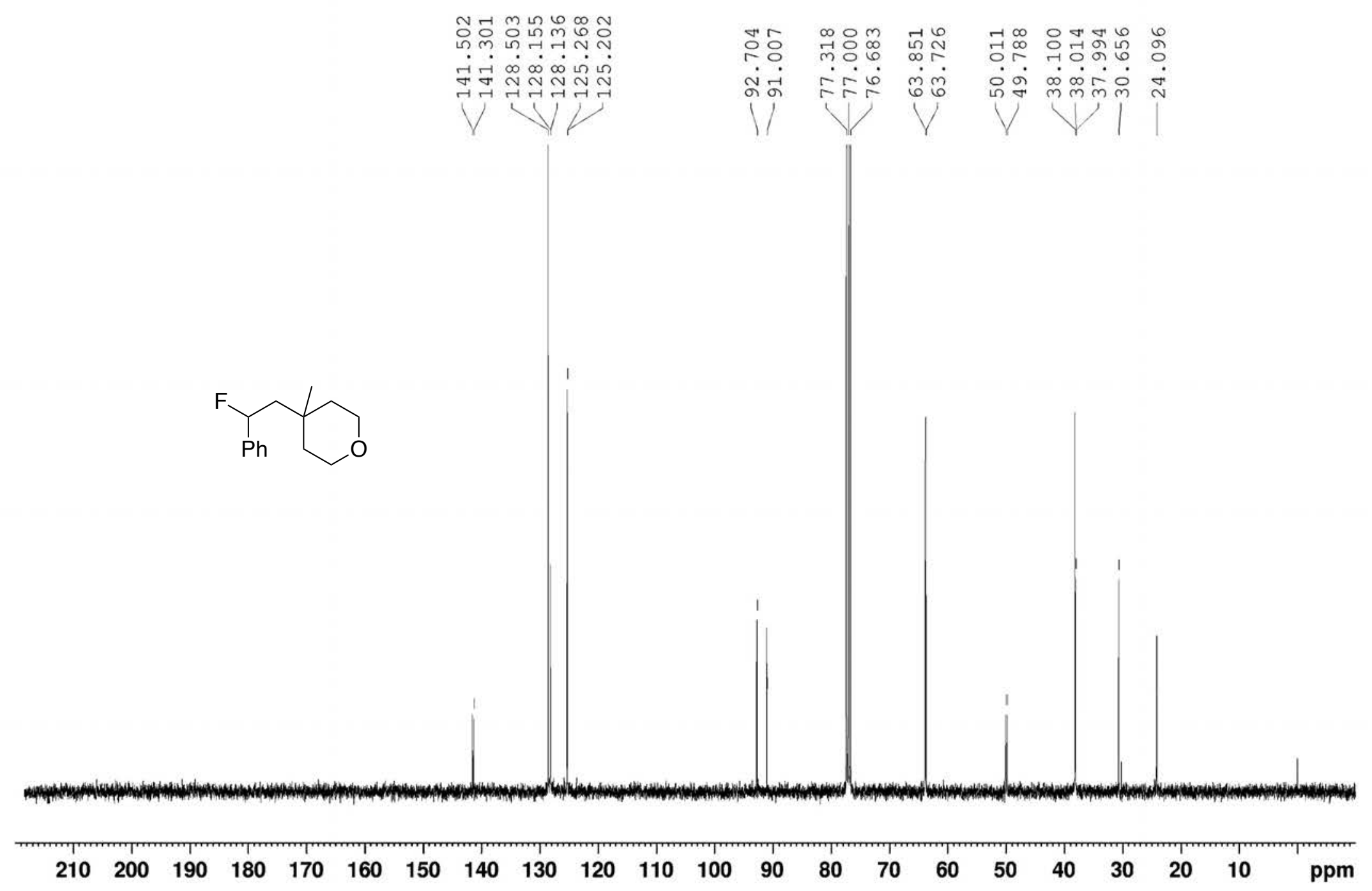

${ }^{13} \mathrm{C}$ NMR spectrum of 13aah $\left(100.6 \mathrm{MHz}, \mathrm{CDCl}_{3}\right)$ 


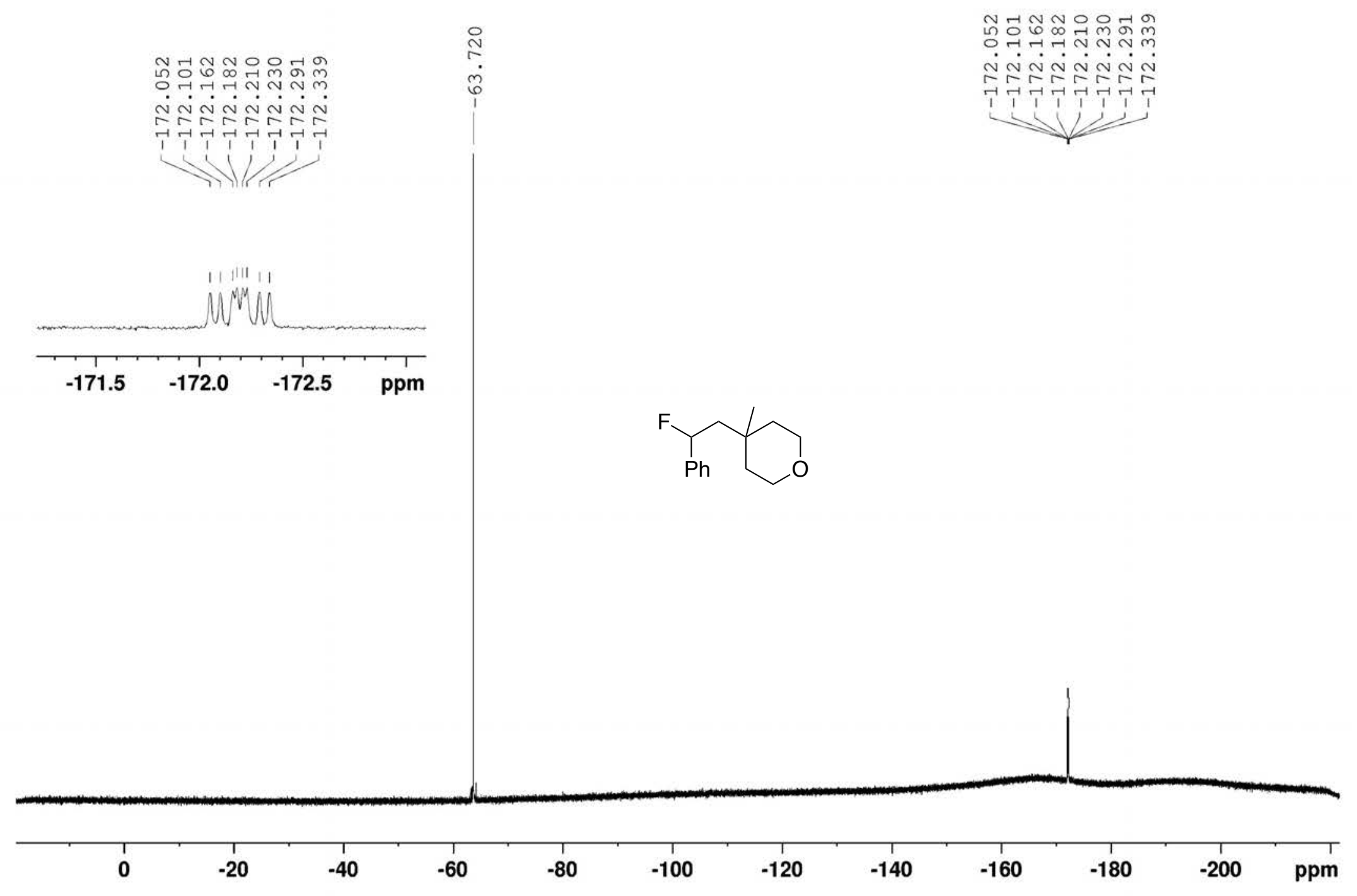

${ }^{19} \mathrm{~F}$ NMR spectrum of 13aah $\left(376.5 \mathrm{MHz}, \mathrm{CDCl}_{3}\right)$ 


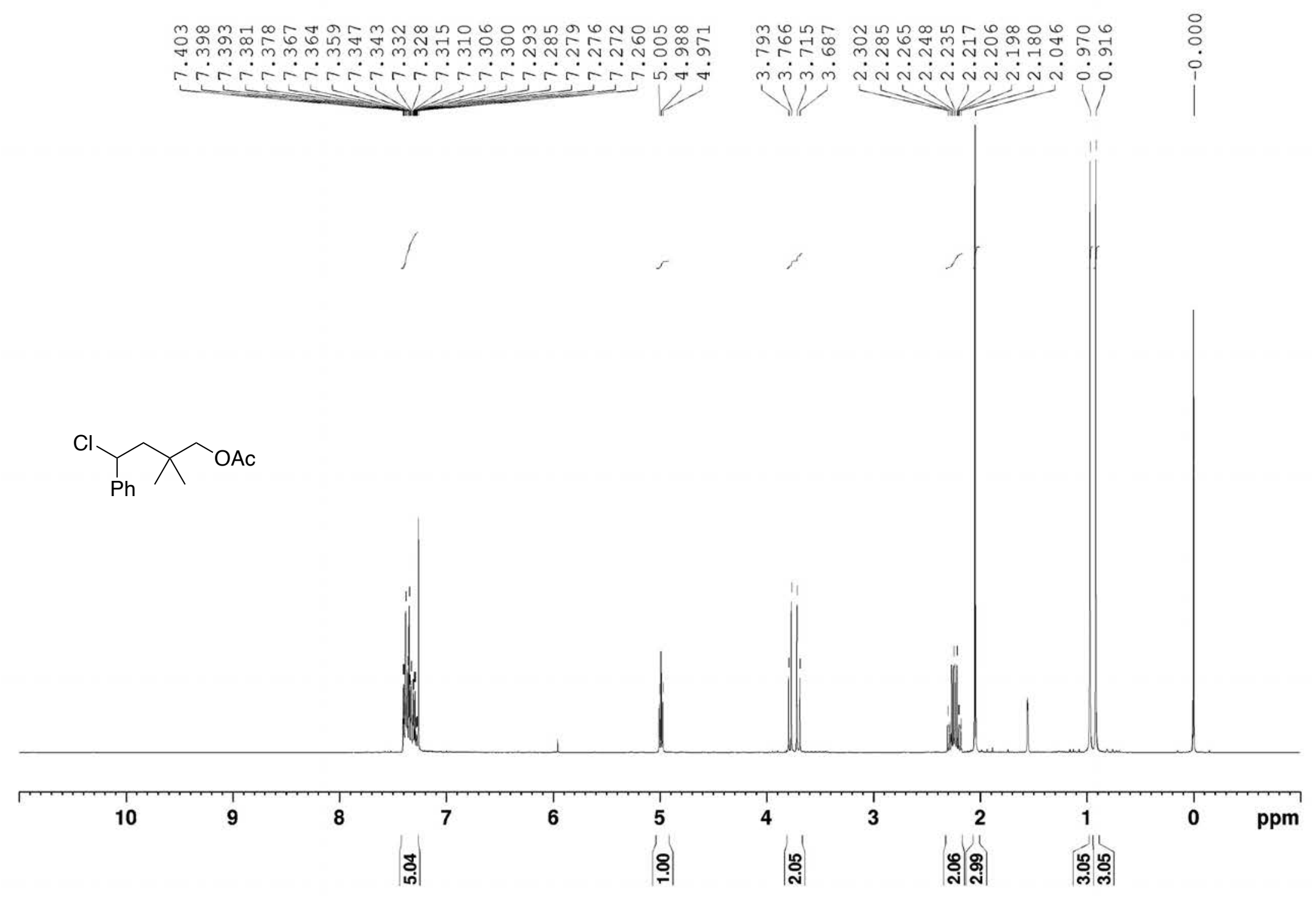

${ }^{1} \mathrm{H}$ NMR spectrum of $\mathbf{1 4 a a b}\left(400 \mathrm{MHz}, \mathrm{CDCl}_{3}\right)$ 


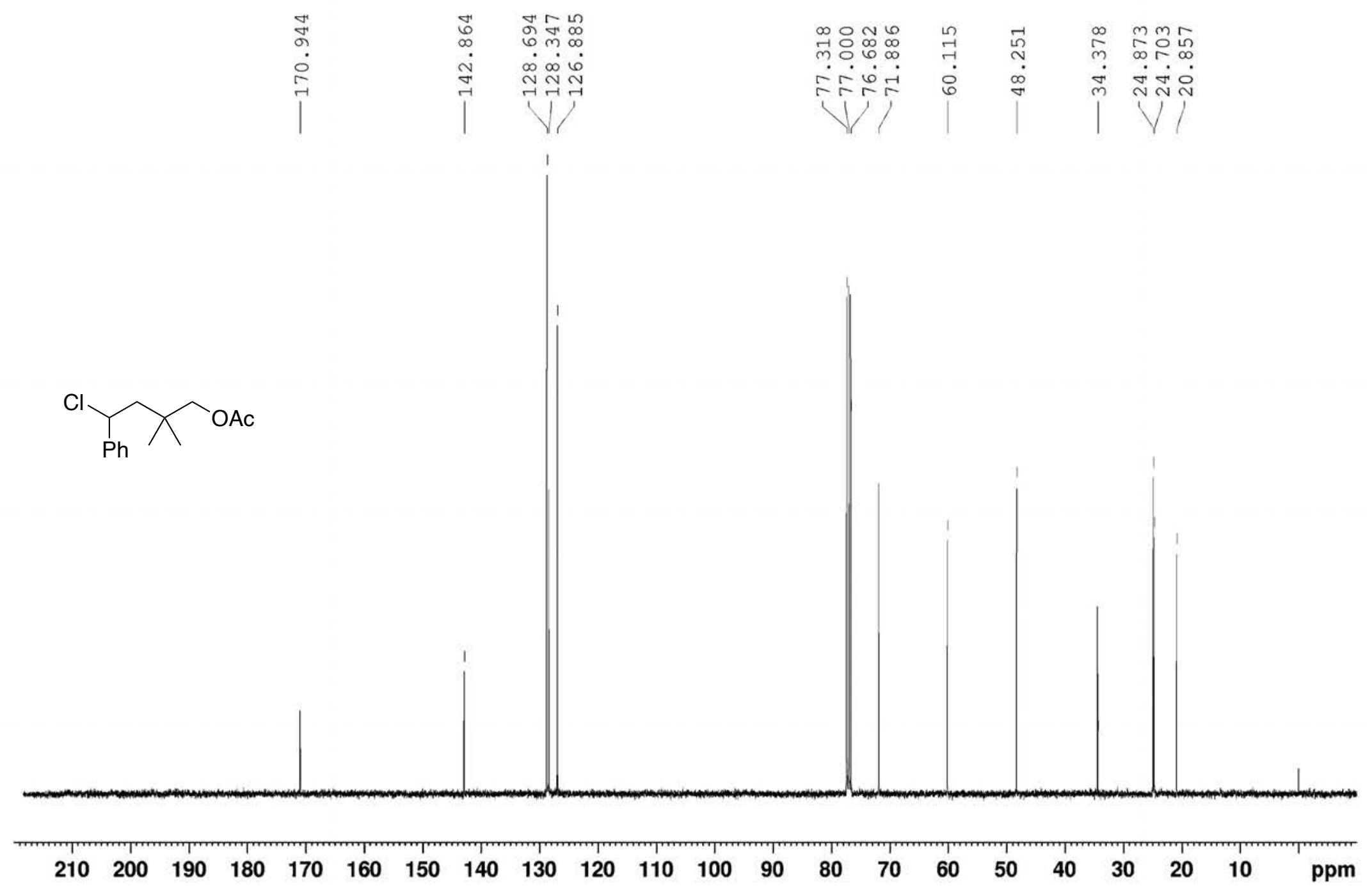

${ }^{13} \mathrm{C}$ NMR spectrum of $\mathbf{1 4 a a b}\left(100.6 \mathrm{MHz}, \mathrm{CDCl}_{3}\right)$ 
우유

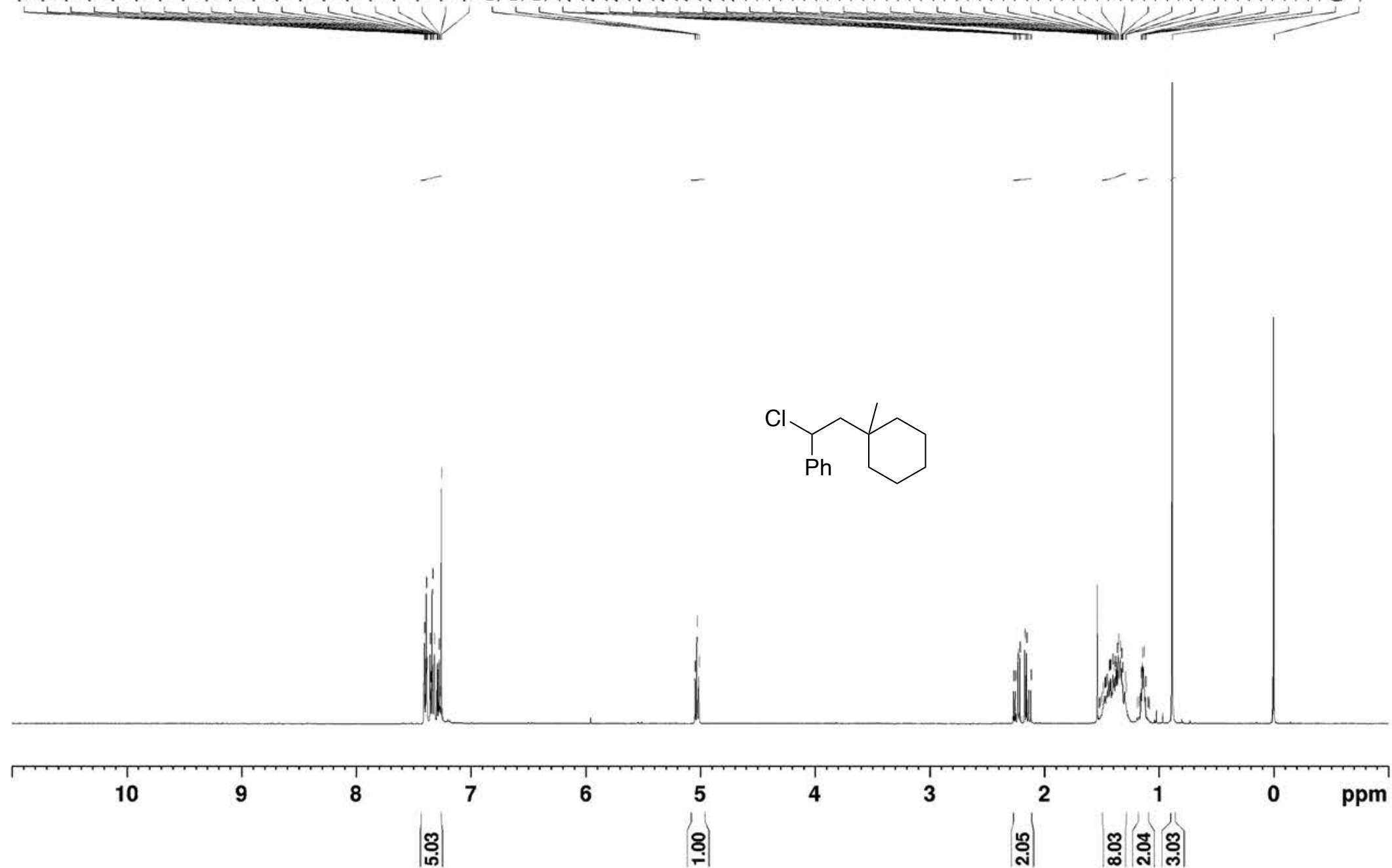

${ }^{1} \mathrm{H}$ NMR spectrum of 14aaf (400 MHz, $\mathrm{CDCl}_{3}$ ) 


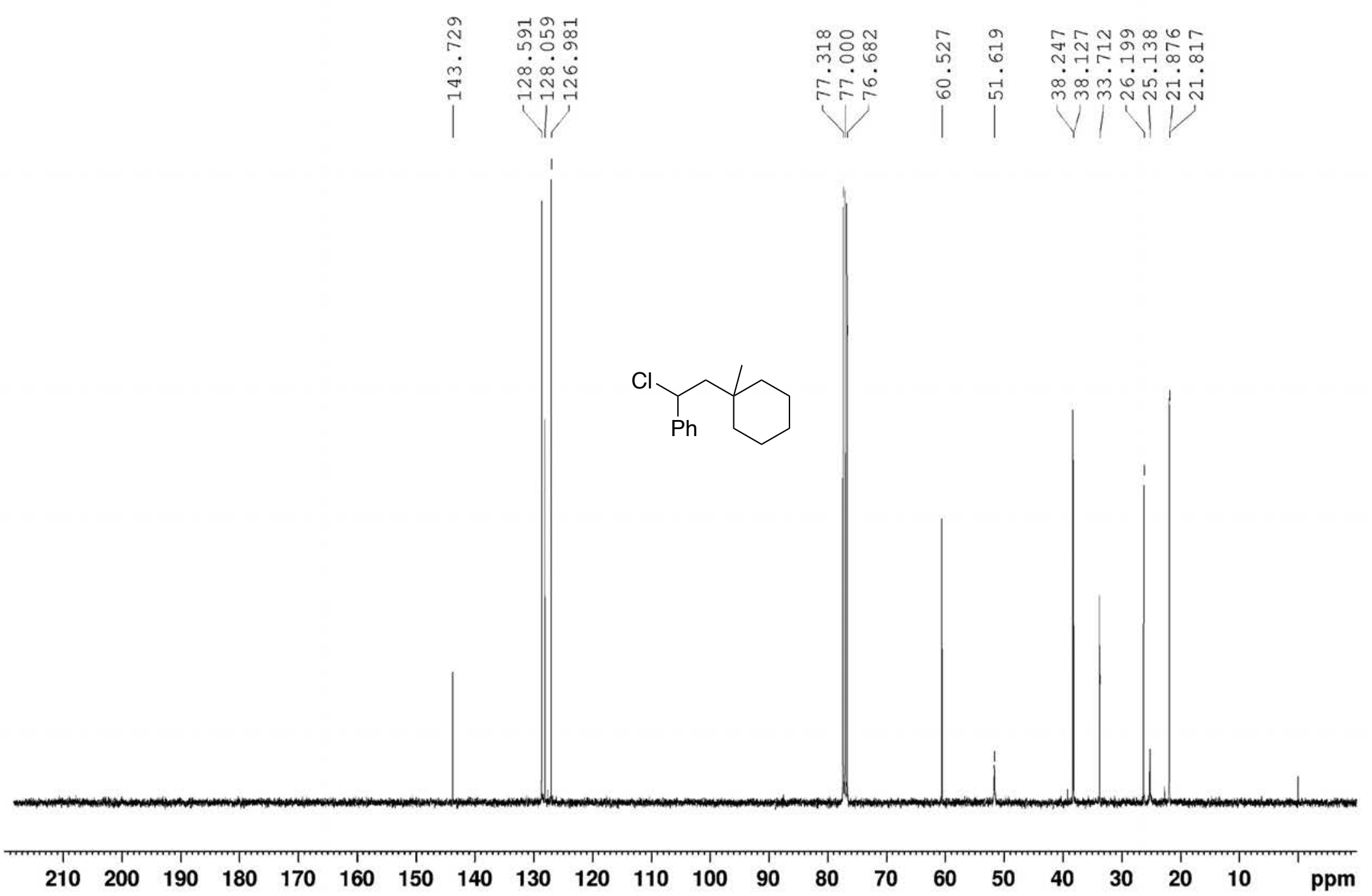

${ }^{13} \mathrm{C}$ NMR spectrum of $14 a a f\left(100.6 \mathrm{MHz}, \mathrm{CDCl}_{3}\right)$ 
야요

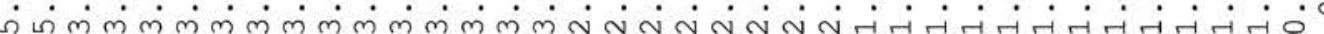

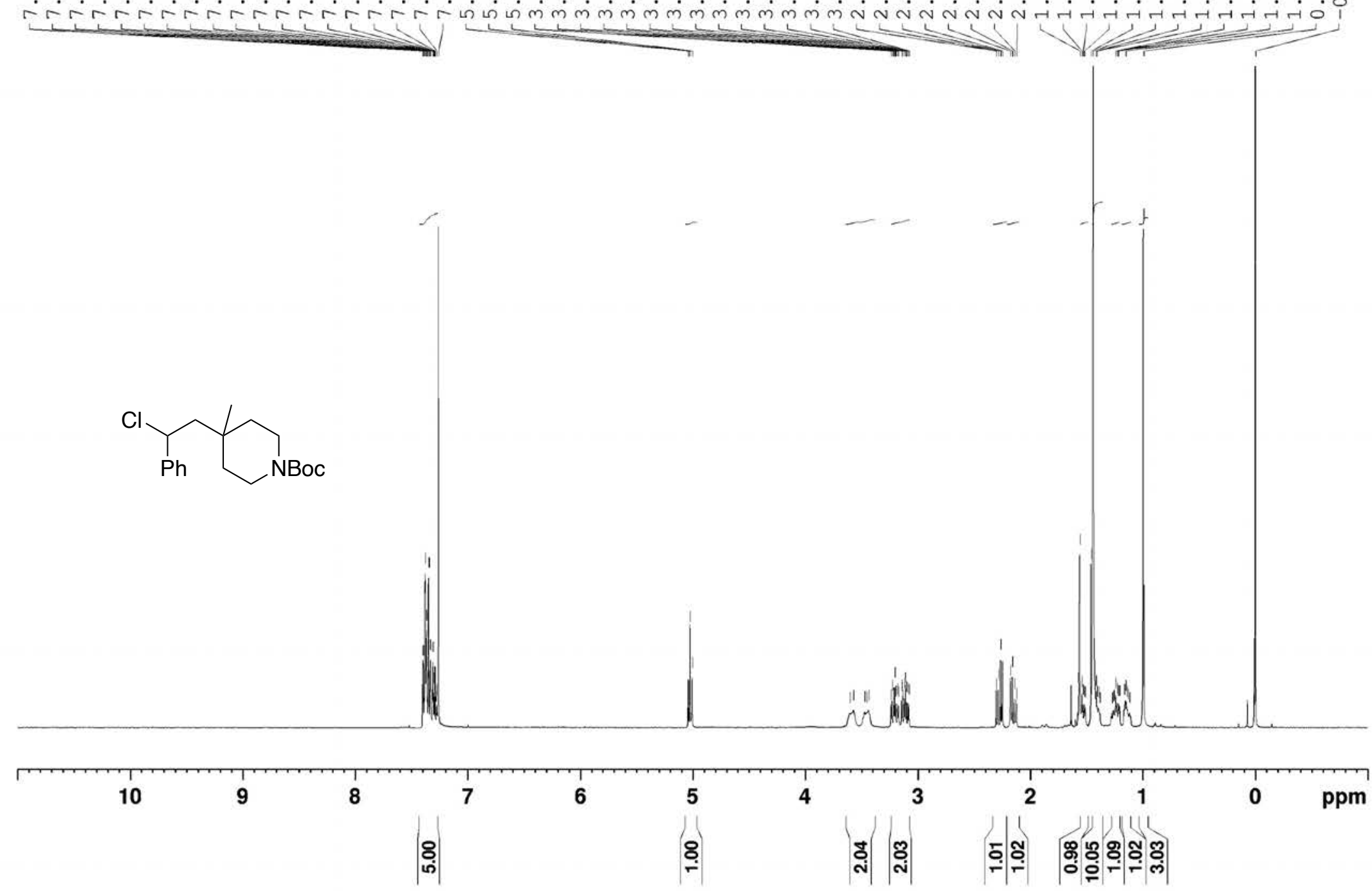

${ }^{1} \mathrm{H}$ NMR spectrum of $\mathbf{1 4 a a g}\left(400 \mathrm{MHz}, \mathrm{CDCl}_{3}\right)$ 


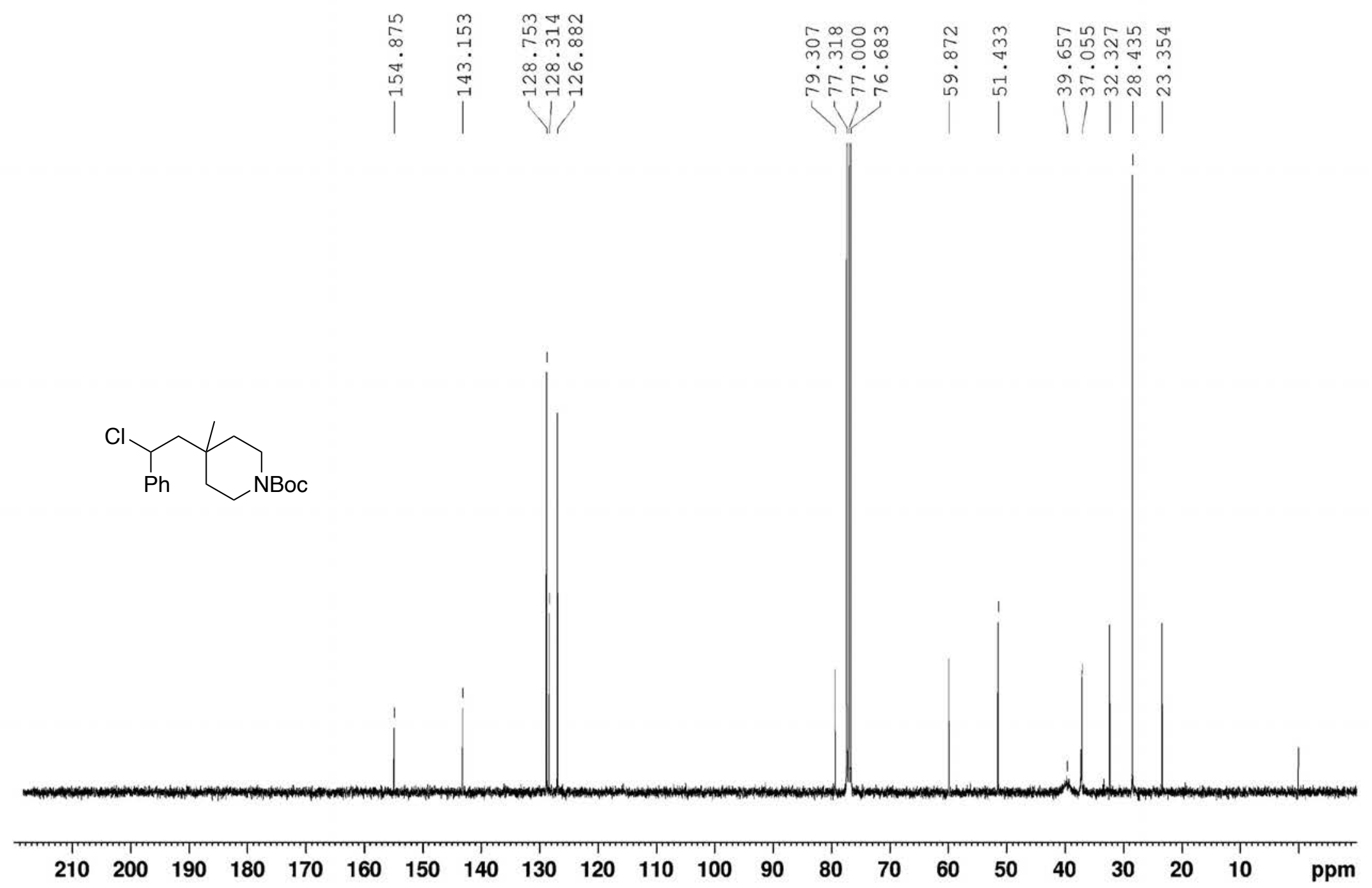

${ }^{13} \mathrm{C}$ NMR spectrum of $\mathbf{1 4 a a g}\left(100.6 \mathrm{MHz}, \mathrm{CDCl}_{3}\right)$ 


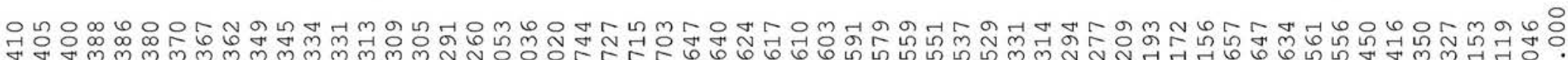

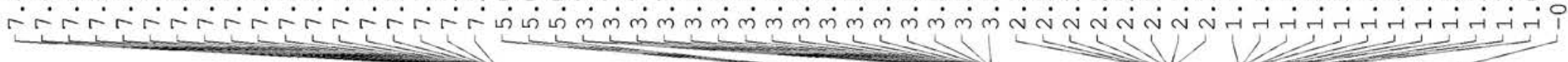

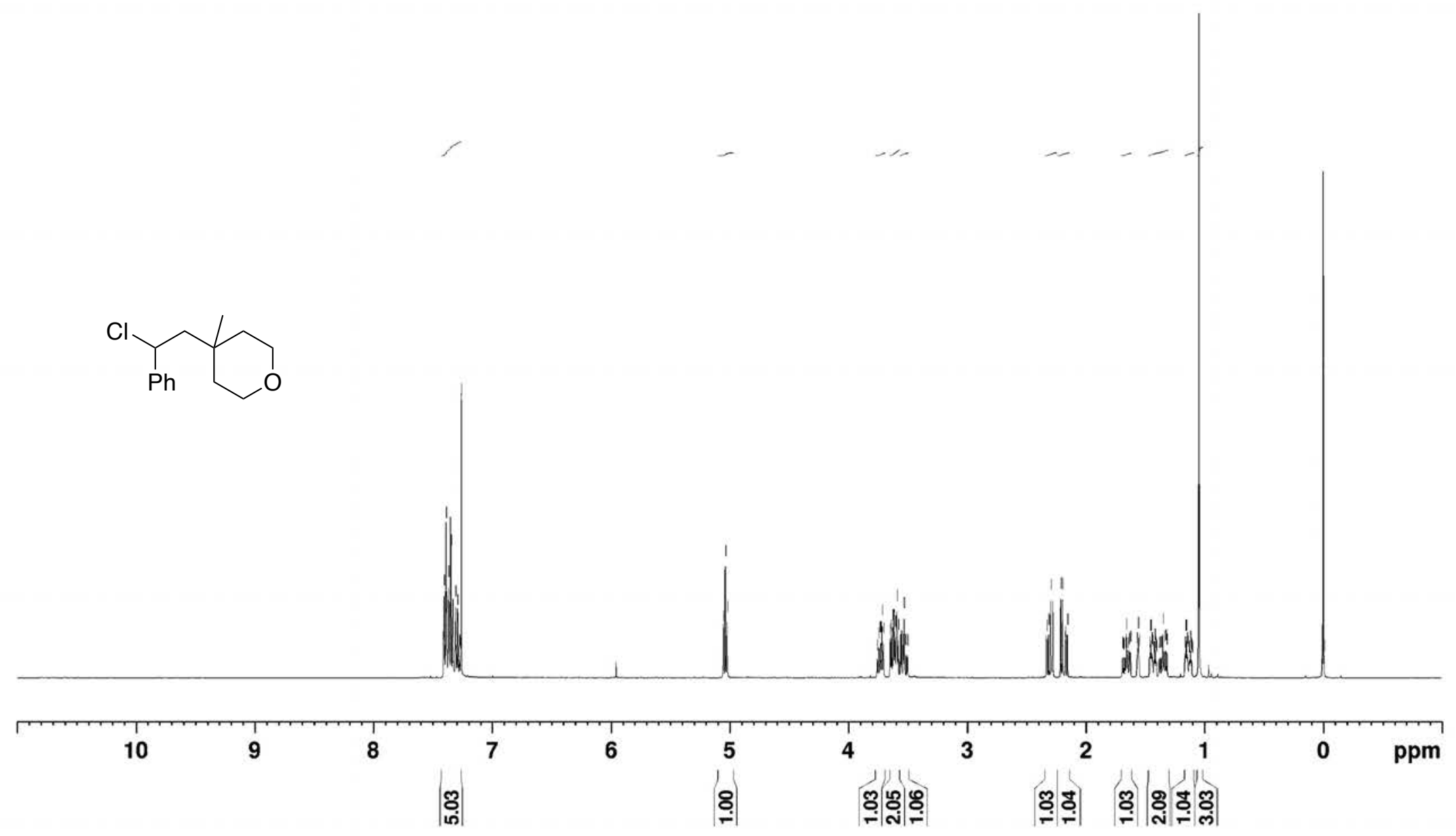

${ }^{1} \mathrm{H}$ NMR spectrum of $\mathbf{1 4 a a h}\left(400 \mathrm{MHz}, \mathrm{CDCl}_{3}\right)$ 


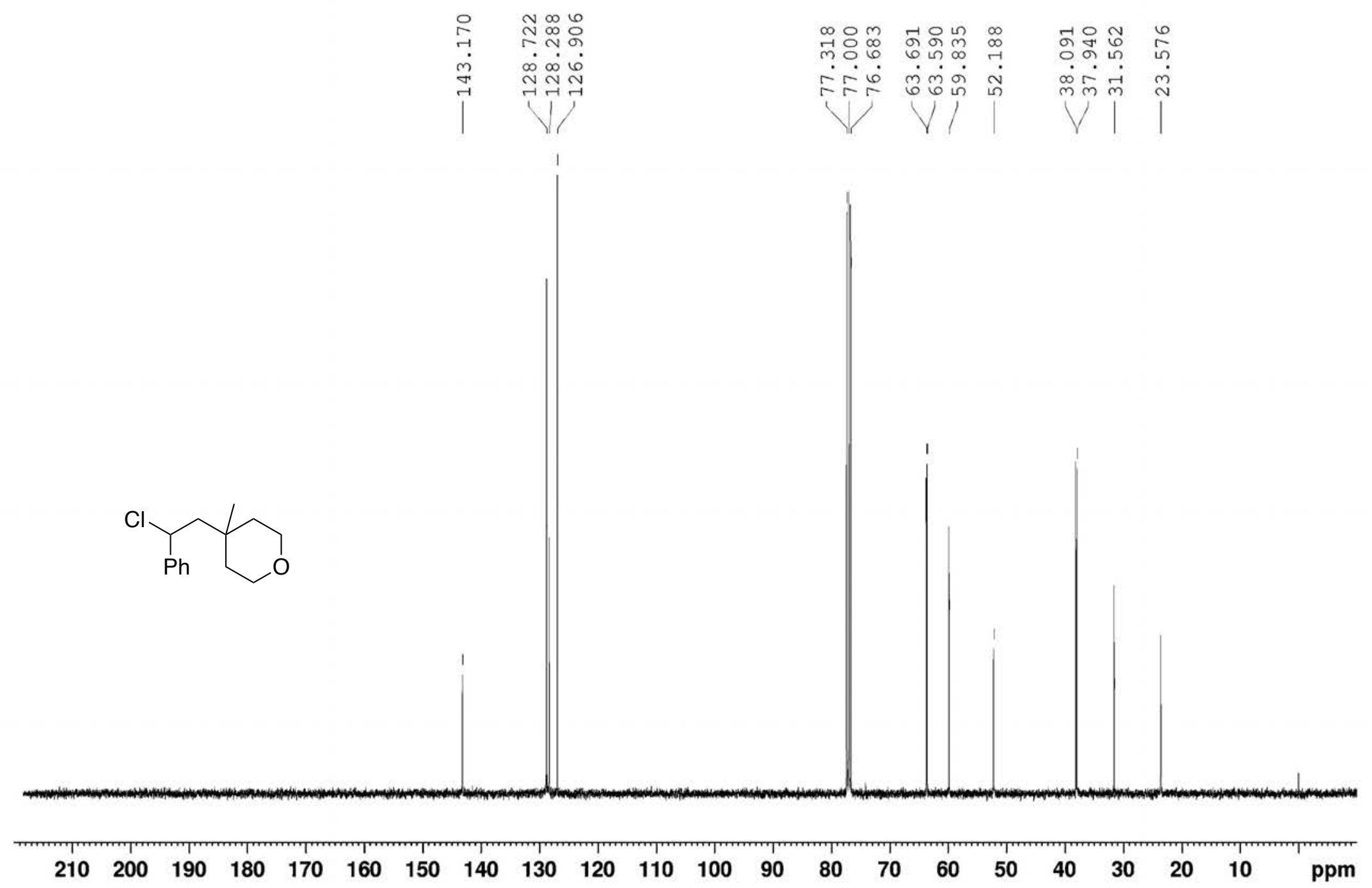

${ }^{13} \mathrm{C}$ NMR spectrum of $\mathbf{1 4 a a h}\left(100.6 \mathrm{MHz}, \mathrm{CDCl}_{3}\right)$ 